Supporting Information

\title{
Synthesis and Photophysical Study of Heteropolycyclic and Carbazole Motif: Nickel-Catalyzed Chelate-Assisted Cascade C-H Activations/Annulations
}

Namrata Prusty ${ }^{\dagger}$, Shyam Kumar Banjare ${ }^{\dagger}$, Smruti Ranjan Mohanty, Tanmayee Nanda, Komal Yadav, and Ponneri C. Ravikumar*

School of Chemical Sciences, National Institute of Science Education and Research (NISER) Bhubaneswar, HBNI, Jatani, Khurda 752050, Odisha, India

$\left({ }^{\dagger}\right.$ Contributed equally)

E-mail:pcr@niser.ac.in 
1. General information \& List of abbreviation

2. Experimental details

2.1 General procedure for reduction of indoles to indolines

2.2 General procedure for preparation of $N$-protected indolines and indoles

2.3 General procedure for the preparation of internal alkynes

2.4 General reaction procedure for $\mathrm{C}(6)-\mathrm{H} \& \mathrm{C}(7)-\mathrm{H}$ activation of $N$-pyridyl indoline with diphenyl acetylene as coupling partner

2.5 General reaction procedure for $\mathrm{C}(2)-\mathrm{H} \& \mathrm{C}(3)-\mathrm{H}$ activation of $\mathrm{N}$-pyridyl indole with diphenyl acetylene as coupling partner

3. Mechanistic Investigation

$3.1 \quad H / D$ exchange studies

3.2 Competition studies with coupling partners

3.3 Detection of Stilbene in the reaction

3.4 Detection of deuterated-Stilbene in the reaction

4. Control experiments

4.1 Synthesis of 3aa in presence of BHT

4.2 Synthesis of 3aa in presence of TEMPO

5. Catalytic cycle

6. Synthetic Applications

$6.11 \mathrm{mmol}$ scale reaction for the synthesis of $\mathbf{3 b a}$

$6.21 \mathrm{mmol}$ scale reaction for the synthesis of $5 \mathbf{b a}$

6.3 DDQ oxidation of $\mathbf{3 b a}$

6.4 Directing group removal of 6

$\begin{array}{lll}6.5 & \text { Directing group removal of } 3 \mathbf{b a} & \mathrm{S} 17\end{array}$

$\begin{array}{ll}6.6 & \text { Directing group removal of } 5 \mathbf{b a} \\ \text { S17 }\end{array}$

$\begin{array}{lll}6.7 & \text { Further annulation of product } 6 & \text { S18 }\end{array}$

$\begin{array}{ll}6.8 \text { Unsuccessful coupling partners } & \text { S18 }\end{array}$

$\begin{array}{ll}\text { 7. References } & \text { S19 }\end{array}$

8. Experimental characterization data for the products S20-S43

9. Copies of NMR spectra for the products and the alkynes $\left({ }^{1} \mathrm{H},{ }^{13} \mathrm{C}\left\{{ }^{1} \mathrm{H}\right\} \&{ }^{19} \mathrm{~F} \quad\right.$ S44-S98 NMR)

10. X-ray data of compound 5ra 


\section{General Information: ${ }^{1}$}

Reactions were performed using oven dried borosil seal-tube glass vial with Teflon-coated magnetic stirring bars under $\mathrm{N}_{2}$ atmosphere. Column chromatography was done by using 100200 and 230-400 mesh size silica gel of Acme synthetic chemicals Company. A gradient elution was performed by using distilled petroleum ether and ethyl acetate. TLC plates were detected under UV light at $254 \mathrm{~nm} .{ }^{1} \mathrm{H}$ NMR, ${ }^{13} \mathrm{C}$ NMR \& ${ }^{19} \mathrm{~F}$ NMR were recorded on Bruker AV 400 and $700 \mathrm{MHz}$ spectrometers using $\mathrm{CDCl}_{3}$ as the deuterated solvent. ${ }^{2}$ Chemical shifts $(\delta)$ are reported in ppm relative to the residual solvents $\left(\mathrm{CHCl}_{3}\right)$ signal $\left(\delta=7.26\right.$ for ${ }^{1} \mathrm{H} \mathrm{NMR}$ and $\delta=77.36$ for ${ }^{13} \mathrm{C}$ NMR). Data for ${ }^{1} \mathrm{H}$ NMR spectra are reported as follows: chemical shift (multiplicity, coupling constants, number of hydrogen). Abbreviations are as follows: s (singlet), d (doublet), t (triplet), q (quartet), quint (quintet), m (multiplet), dd (double doublet), br (broad signal), $J$ (coupling constants) in $\mathrm{Hz}$ (hertz). High-Resolution Mass Spectrometry (HRMS) data was recorded using micro TOF Q-II mass spectrometer using methanol as solvent. IR spectra were recorded on a FTIR system and values are reported in frequency of absorption $\left(\mathrm{cm}^{-1}\right)$. Melting point was performed using Stuart ${ }^{\mathrm{TM}}$ melting point apparatus SMP10. X-ray analysis was conducted using Rigaku Smartlab X-ray diffractometer, Steadystate absorption and emission spectra are recorded using a Jasco V-730 spectrophotometer and Shimadzu RF-6000 fluorescence spectrophotometer (Shimadzu Corporation), respectively. For emission spectra, samples were excited at $375 \mathrm{~nm}$ at SCS, NISER. For the measurement of time-resolved fluorescence, a time-correlated single-photon counting (TCSPC) spectrometer (Edinburgh, OB920) was used. Reagents and starting materials were purchased from Sigma Aldrich, Alfa Aesar, TCI, Avra, Spectrochem and other commercially available sources and used without further purification unless otherwise noted.

\section{Abbreviation:}

$\mathrm{Et}_{3} \mathrm{~N}$ (Triethylamine), $\mathrm{NaBH}_{3} \mathrm{CN}$ (Sodium cyano borohydride), $\mathrm{AcOH}$ (Acetic acid), $\mathrm{CuI}$ (Copper iodide), $\mathrm{K}_{2} \mathrm{CO}_{3}$ (Potassium carbonate), $\mathrm{Ni}(\mathrm{OTf})_{2}$ (Nickel trifluoromethanesulfonate), $\mathrm{PPh}_{3}$ (Triphenyl phosphine), NaI (Sodium iodide), Py (Pyridine), Pym (Pyrimidine), DBU (Diazabicycloundecane), EtOAc (Ethyl acetate), DCM (Dichloromethane), DCE (1,2Dichloroethane), DMF (Dimethylformamide), TFE (Trifluoroethanol), TFT (Trifluorotoluene), THF (Tetrahydrofuran), NMP ( $N$-methyl pyrolidine), rt (Room temperature), TEMPO ((2,2,6,6-Tetramethylpiperidin-1-yl)oxyl), BHT (Butylated hydroxytoluene), DDQ (2,3-Dichloro-5,6-dicyano-1,4-benzoquinone). 


\section{Experimental procedure:}

\subsection{General procedure for reduction of indoles to indolines: ${ }^{3}$}<smiles>[R]=[X]1ccc2cc[nH]c2c1</smiles>

(1 equiv)

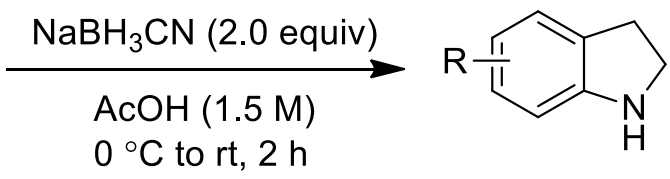

$\mathbf{R}=\mathrm{Me}, \mathrm{OMe}, \mathrm{F}, \mathrm{Cl}, \mathrm{Br}, \mathrm{I}, \mathrm{NO}_{2}, \mathrm{CN}$

To a solution of indole ( $40 \mathrm{mmol}, 1$ equiv) in acetic acid $(1.5 \mathrm{M}, 27 \mathrm{~mL})$ was added in portions sodium cyanoborohydride ( $80 \mathrm{mmol}, 2$ equiv) at $0{ }^{\circ} \mathrm{C}$, and the mixture was stirred at room temperature. After $2 \mathrm{~h}$, water $(50 \mathrm{~mL})$ was added at $0{ }^{\circ} \mathrm{C}$ and the mixture was basified with $\mathrm{NaOH}$ (5 $\mathrm{N}$ aqueous solution). The organic material was extracted with $\mathrm{CH}_{2} \mathrm{Cl}_{2}(100 \mathrm{~mL} \times 3)$, washed with brine, dried over anhydrous $\mathrm{MgSO}_{4}$ and concentrated using a rotary evaporator to give crude reaction mixture. The column chromatography gives the pure product.

\subsection{General procedure for the preparation of $N$-protected indolines and indoles: ${ }^{4}$}

(a) 1-(pyrimidin-2-yl)indolines were prepared by following reported procedure ${ }^{4 a}$

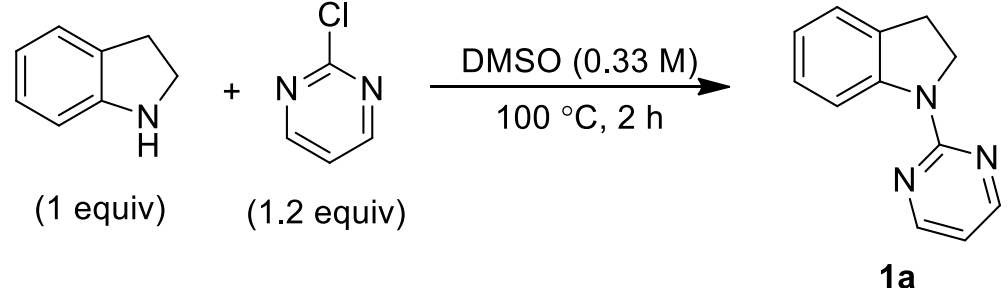

To a well-stirred solution of indoline ( $4.2 \mathrm{mmol}, 1$ equiv) in DMSO $(0.33 \mathrm{M}, 13 \mathrm{~mL})$ at room temperature was added 2-chloropyrimidine ( $5 \mathrm{mmol}, 1.2$ equiv). The reaction was refluxed at $100{ }^{\circ} \mathrm{C}$ for $2 \mathrm{~h}$. After $2 \mathrm{~h}$, the reaction flask was cooled to room temperature. After completion of the reaction (as monitored by TLC analysis), it is cooled to room temperature and worked up with cold water. The product was extracted with EtOAc (3 x $20 \mathrm{~mL})$ and dried over anhydrous $\mathrm{Na}_{2} \mathrm{SO}_{4}$. The organic phase was concentrated in vacuum to obtain the crude mixture which was further purified by column chromatography (using 5\% EtOAc/hexane) giving the product as white solid (99\% yield). 
(b) 1-(pyridin-2-yl)indolines were prepared by following reported procedure ${ }^{4 b}$

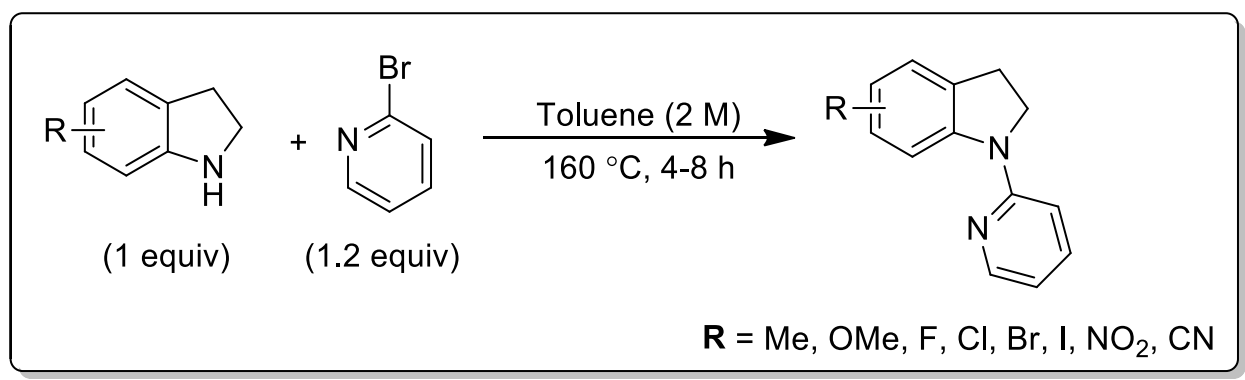

To an oven dried $25 \mathrm{~mL}$ round bottom flask charged with indoline ( $8.4 \mathrm{mmol}, 1$ equiv) were added 2-bromopyridine (10 mmol, 1.2 equiv) and toluene (2 M, $4.2 \mathrm{~mL})$. The reaction was stirred at $160{ }^{\circ} \mathrm{C}$ for $8 \mathrm{~h}$. The reaction mixture was diluted with EtOAc and washed with saturated $\mathrm{NaHCO}_{3}$ solution. The organic solution was dried over anhydrous $\mathrm{Na}_{2} \mathrm{SO}_{4}$. The organic phase was concentrated in vacuum to obtain the crude mixture which was further purified by column chromatography (using 5\% EtOAc/hexane) giving the pure product.

(c) General and modified procedure for preparation of 1-(pyridin-2-yl)-1H-indole: ${ }^{4 c, d}$

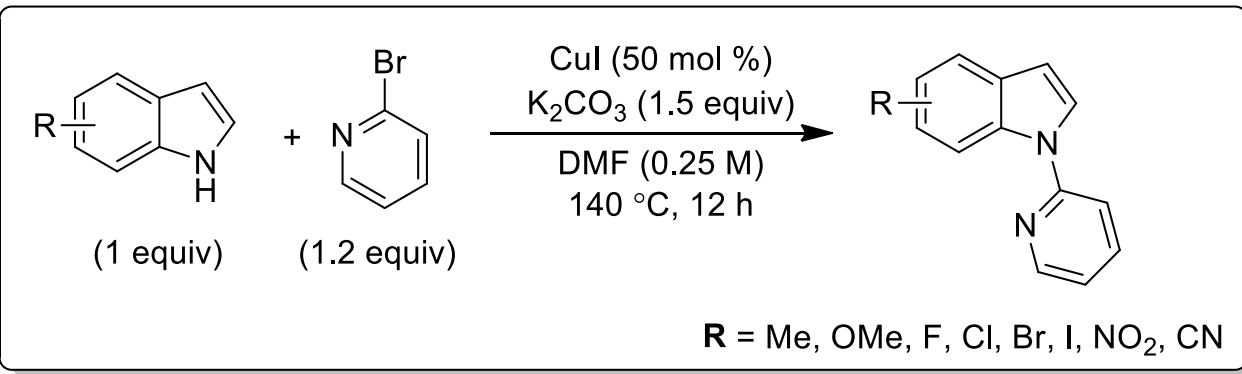

$\mathrm{N}$-pyridyl indoles were prepared by following reported procedure. To an oven dried $25 \mathrm{~mL}$ sealed tube charged with a stirring bar, indole ( $1.7 \mathrm{mmol}, 1$ equiv), copper iodide ( $0.86 \mathrm{mmol}$, 0.5 equiv), $\mathrm{K}_{2} \mathrm{CO}_{3}$ (2.6 mmol, 1.5 equiv) were added sequentially under nitrogen atmosphere. Then 2-bromopyridine ( $1.9 \mathrm{mmol}, 1.1$ equiv) was added to the reaction mixture followed by addition of DMF solvent $(0.25 \mathrm{M})$. The reaction was allowed to stir at $140{ }^{\circ} \mathrm{C}$ in a pre-heated aluminum block until the reaction completed. After completion of the reaction (as monitored by TLC analysis), it is cooled to room temperature and worked up with cold water. The product was extracted with EtOAc ( 3 × $20 \mathrm{~mL})$ and dried over anhydrous $\mathrm{Na}_{2} \mathrm{SO}_{4}$. The organic phase was concentrated in vacuum to obtain the crude mixture which was further purified by column chromatography (using 5\% EtOAc/hexane) giving the pure product. 


\subsection{General procedure for preparation of internal alkynes: ${ }^{5}$}

Symmetrical alkynes $\mathbf{2 b}, \mathbf{2 c}, \mathbf{2 d}, \mathbf{2 e}, \mathbf{2 f}$ and $\mathbf{2 i}$ were prepared with a little modification in the reported procedure.

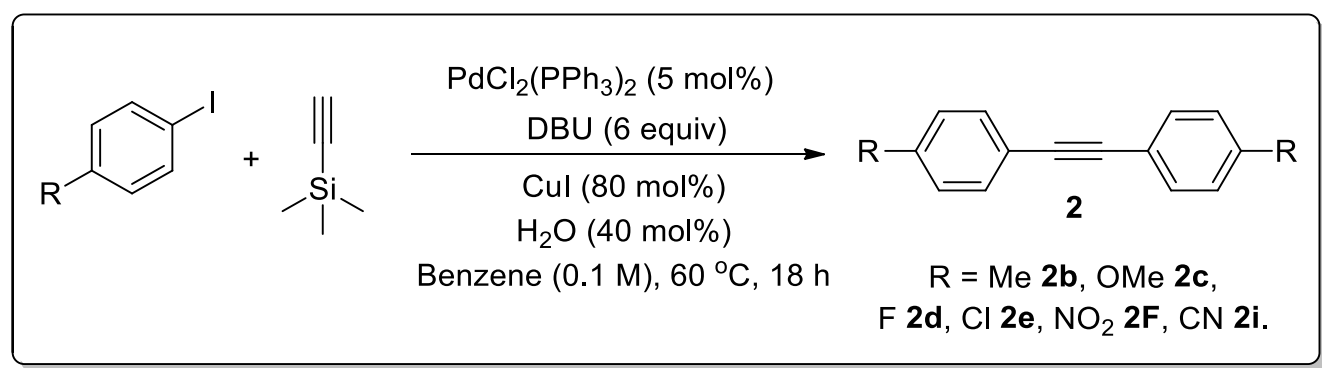

To an oven dried sealed tube charged with a stirring bar, dry benzene $(0.1 \mathrm{M})$, aryl iodide $(2.8$ equiv), trimethylsilylacetylene (1 equiv), $\mathrm{PdCl}_{2}\left(\mathrm{PPh}_{3}\right)_{2}$ (4.5 mol \%), $\mathrm{CuI}(80 \mathrm{~mol} \%), \mathrm{DBU}$ (6 equiv) and distilled water (40 mol \%) were added sequentially under nitrogen atmosphere. The solution was stirred at $\mathrm{rt}$ for 20-30 minute and then kept it in a pre-heated aluminum block at $60{ }^{\circ} \mathrm{C}$ for $15-20 \mathrm{~h}$. After completion of the reaction (as monitored by TLC analysis), the reaction mixture was quenched with $10 \% \mathrm{HCl}(10 \mathrm{~mL})$. Then the crude mixture was extracted with EtOAc and washed with saturated $\mathrm{NaCl}$. After drying over anhydrous $\mathrm{Na}_{2} \mathrm{SO}_{4}$, the crude mixture was purified by column chromatography using EtOAc/hexane mixture on silica gel to furnish the pure product.

\subsection{General reaction procedure for $\mathrm{C}(6)-\mathrm{H} \& \mathrm{C}(7)$-H activation of 1-(pyridin-2- yl)indolines with diphenyl acetylene as coupling partner:}

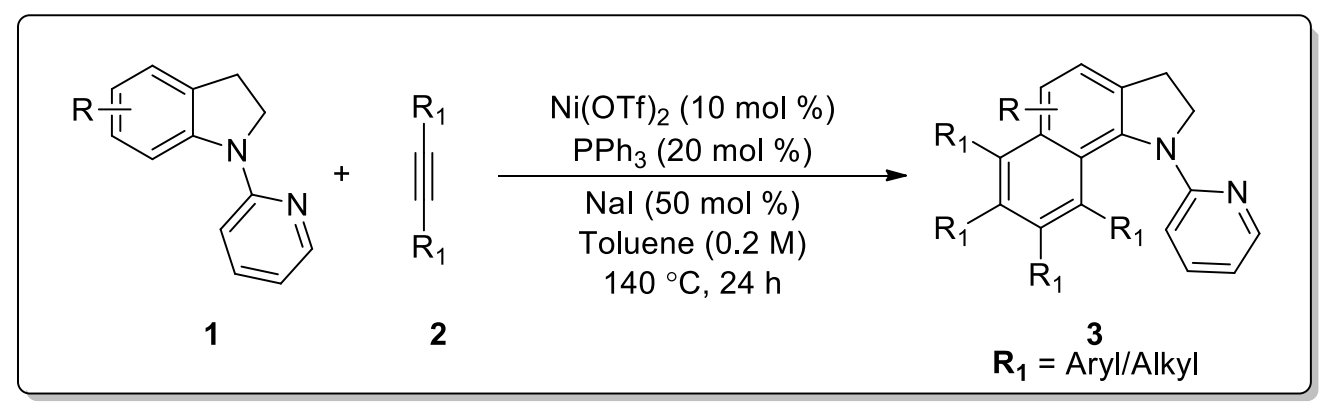

To an oven dried sealed tube charged with a stirring bar under $\mathrm{N}_{2}$ atmosphere, the 1-(pyridin2-yl)indoline 1 (0.1 mmol), diphenylacetylene 2 (4 equiv), $\mathrm{PPh}_{3}(20 \mathrm{~mol} \%), \mathrm{NaI}(50 \mathrm{~mol} \%)$ were added and sealed. The sealed tube was high-vacuumed and refilled with $\mathrm{N}_{2}$. Then $\mathrm{Ni}(\mathrm{OTf})_{2}(10 \mathrm{~mol} \%)$ and degassed toluene solvent $(0.2 \mathrm{M})$ were added to the reaction mixture inside the glove box. Then the sealed tube was taken out of the glove box and reaction mixture was vigorously stirred at $140{ }^{\circ} \mathrm{C}$ on preheated aluminum block for $24 \mathrm{~h}$. After $24 \mathrm{~h}$ (upon completion of reaction as monitored by TLC analysis), the reaction mixture was cooled to room 
temperature and diluted with EtOAc or DCM and passed through a short celite pad. The solvent was evaporated under reduced pressure and the residue was purified by column chromatography using EtOAc/hexane mixture on silica gel to give the pure product $\mathbf{3}$.

\subsection{General reaction procedure for $\mathrm{C}(2)-\mathrm{H} \& \mathrm{C}(3)$-H activation of 1-(pyridin-2-yl)-1H- indole with diphenyl acetylene as coupling partner:}

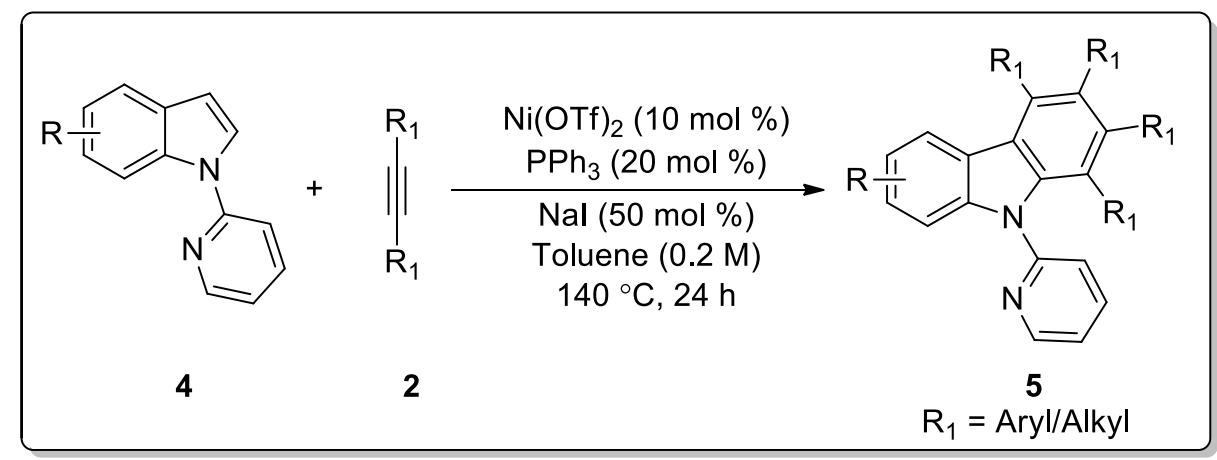

To an oven dried sealed tube charged with a stirring bar under $\mathrm{N}_{2}$ atmosphere, the 1-(pyridin2-yl)-1H-indole 4 (0.1 mmol), diphenylacetylene 2 (4 equiv), $\mathrm{PPh}_{3}(20 \mathrm{~mol} \%), \mathrm{NaI}$ (50 mol $\%$ ), were added and sealed. The sealed tube was high-vacuumed and refilled with $\mathrm{N}_{2}$. Then $\mathrm{Ni}(\mathrm{OTf})_{2}(10 \mathrm{~mol} \%)$ and degassed toluene solvent $(0.2 \mathrm{M})$ were added to the reaction mixture inside the glove box. Then the sealed tube was taken out of the glove box and reaction mixture was vigorously stirred at $140{ }^{\circ} \mathrm{C}$ on preheated aluminum block for $24 \mathrm{~h}$. After $24 \mathrm{~h}$ (upon completion of reaction as monitored by TLC analysis), the reaction mixture was cooled to room temperature and diluted with EtOAc or DCM and passed through a short celite pad. The solvent was evaporated under reduced pressure and the residue was purified by column chromatography using EtOAc/hexane mixture on silica gel to give the pure product $\mathbf{5}$.

\section{Mechanistic Investigation:}

\section{1 $H / D$ exchange studies}

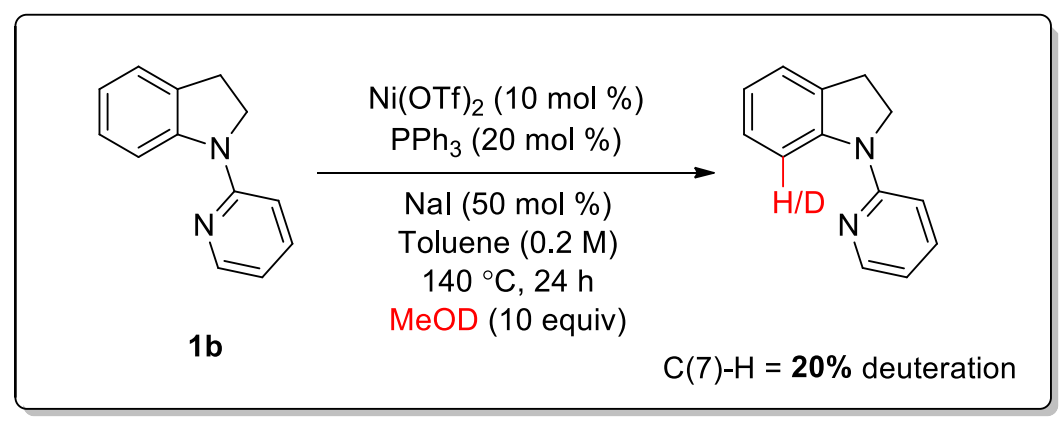

To an oven dried sealed tube charged with a stirring bar under $\mathrm{N}_{2}$ atmosphere, $N$-pyridyl indoline $1 \mathbf{b}(0.1 \mathrm{mmol}), \mathrm{PPh}_{3}(20 \mathrm{~mol} \%), \mathrm{NaI}(50 \mathrm{~mol} \%)$, were added. The sealed tube was high-vacuumed and refilled with $\mathrm{N}_{2}$. Then MeOD (10 equiv) was added to the reaction mixture 
and sealed. Then the $\mathrm{Ni}(\mathrm{OTf})_{2}(10 \mathrm{~mol} \%)$ and degassed toluene solvent $(0.2 \mathrm{M})$ were added to the reaction mixture inside the glove box. Then the sealed tube was taken out of the glove box and reaction mixture was vigorously stirred at $140{ }^{\circ} \mathrm{C}$ on preheated aluminum block for 24 h. After $24 \mathrm{~h}$, the reaction mixture was cooled to room temperature and diluted with EtOAc and passed through a short celite pad. The solvent was evaporated under reduced pressure and the residue was purified by column chromatography using EtOAc/hexane mixture on silica gel to give the pure deuterated product with $20 \% \mathrm{C}(7)-\mathrm{H}$ deuteration.

[C(7)-H dueterated $N$-pyridyl indoline]: The crude reaction mixture was purified by column chromatography using silica gel (100-200 mesh) giving a white solid (9.8 mg, 99\% yield). $\mathbf{R}_{f}$ : 0.40 (10\% EtOAc/hexane).

${ }^{1} \mathbf{H}$ NMR $\left(\mathbf{C D C l}_{3}, \mathbf{4 0 0} \mathbf{M H z}\right): \delta 8.34\left(\mathrm{dd}, J_{l}=4.8 \mathrm{~Hz}, J_{2}=1.2 \mathrm{~Hz}, 1 \mathrm{H}\right), 8.18(\mathrm{~d}, J=8.4 \mathrm{~Hz}$, $1 \mathrm{H}), 7.59-7.55(\mathrm{~m}, 1 \mathrm{H}), 7.19-7.15(\mathrm{~m}, 2 \mathrm{H}), 6.86(\mathrm{t}, J=7.6 \mathrm{~Hz}, 1 \mathrm{H}), 6.78-6.74(\mathrm{~m}, 2 \mathrm{H}), 4.03$ $(\mathrm{t}, J=8.4 \mathrm{~Hz}, 2 \mathrm{H}), 3.20(\mathrm{t}, J=8.4 \mathrm{~Hz}, 2 \mathrm{H})$.

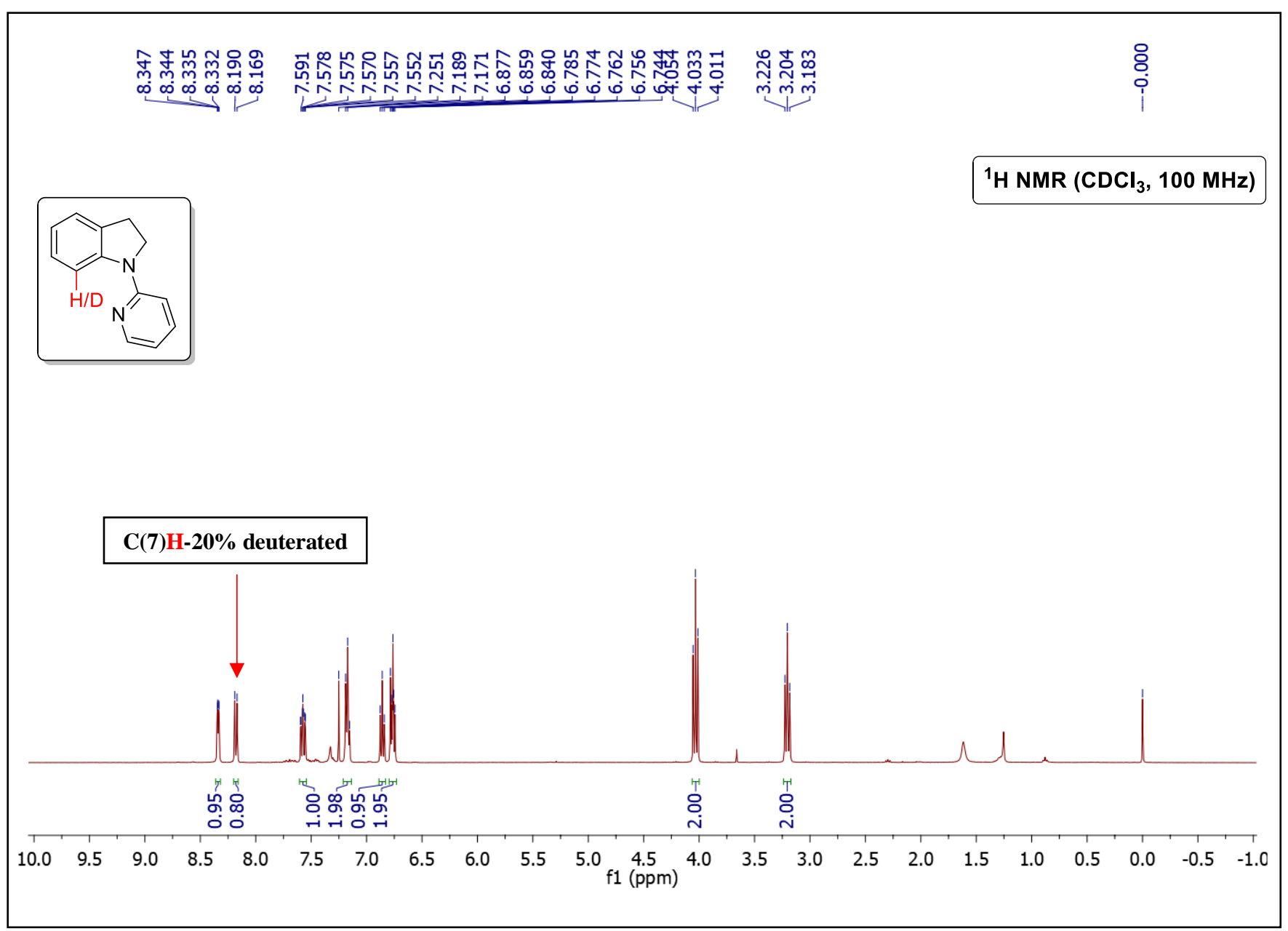




\subsection{Competition studies with coupling partners:}

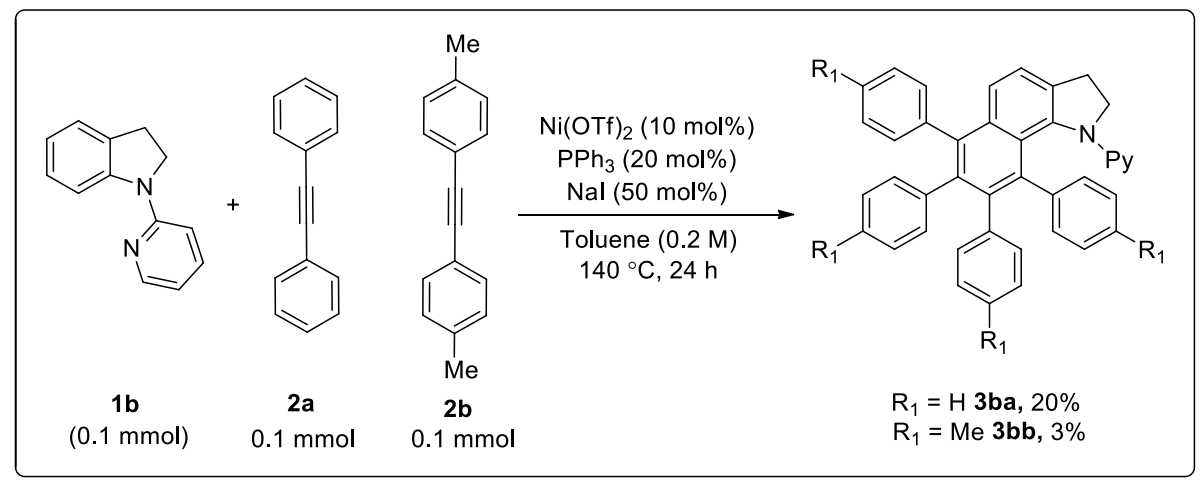

To an oven dried sealed tube charged with a stirring bar under $\mathrm{N}_{2}$ atmosphere, $N$-pyridyl indoline $\mathbf{1 b}$ (20 mg, $0.1 \mathrm{mmol})$, diphenylacetylene $\mathbf{2 a}$ (18 mg, $0.1 \mathrm{mmol}), 1,2$-di-p-tolylethyne 2b (18 mg, $0.1 \mathrm{mmol}), \mathrm{PPh}_{3}(20 \mathrm{~mol} \%), \mathrm{NaI}(50 \mathrm{~mol} \%)$ were added and sealed. The sealed tube was high-vacuumed and refilled with $\mathrm{N}_{2}$. Then $\mathrm{Ni}(\mathrm{OTf})_{2}(10 \mathrm{~mol} \%)$ and degassed toluene solvent $(0.2 \mathrm{M})$ were added to the reaction mixture inside the glove box. Then the sealed tube was taken out of the glove box and reaction mixture was vigorously stirred at $140{ }^{\circ} \mathrm{C}$ on preheated aluminum block for $24 \mathrm{~h}$. After $24 \mathrm{~h}$ (upon completion of reaction as monitored by TLC analysis), the reaction mixture was cooled to room temperature and diluted with EtOAc and passed through a short celite pad. The solvent was evaporated under reduced pressure and the residue was purified by column chromatography using EtOAc/hexane mixture on silica gel to give the pure product $\mathbf{3 b a}$ in $20 \%$ yield $(11 \mathrm{mg})$ and $\mathbf{3 b b}$ in $3 \%$ yield $(2 \mathrm{mg})$.

\subsection{Detection of Stilbene in the reaction:}

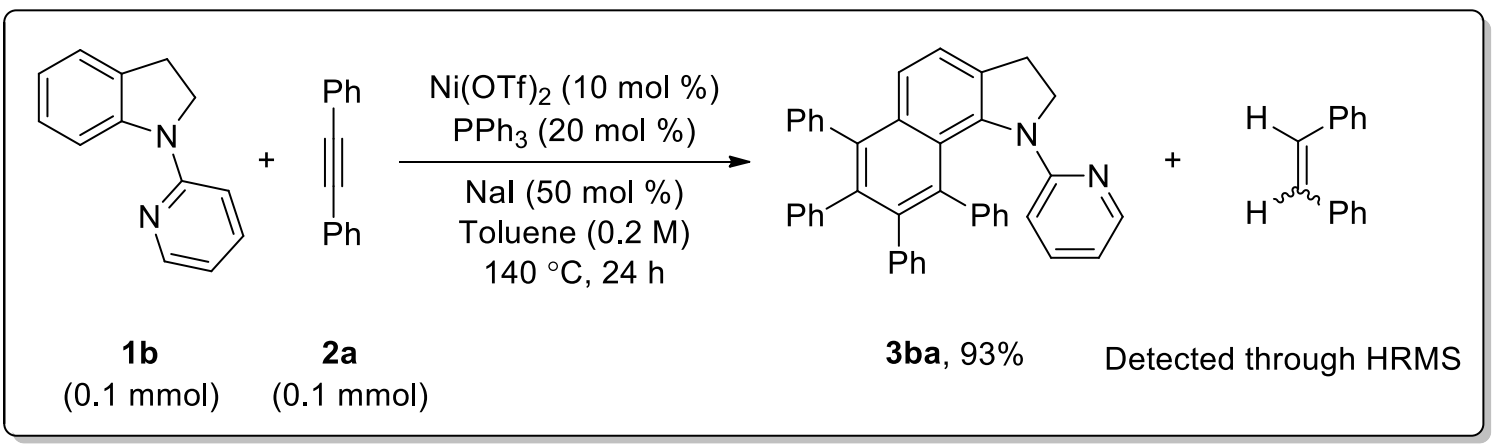

To an oven dried sealed tube charged with a stirring bar under $\mathrm{N}_{2}$ atmosphere, $N$-pyridyl indoline $\mathbf{1 b}$ (20 mg, $0.1 \mathrm{mmol})$, diphenylacetylene $\mathbf{2 a}(18 \mathrm{mg}, 0.1 \mathrm{mmol}), \mathrm{PPh}_{3}(20 \mathrm{~mol} \%)$, $\mathrm{NaI}(50 \mathrm{~mol} \%)$ were added and sealed. The sealed tube was high-vacuumed and refilled with $\mathrm{N}_{2}$. Then $\mathrm{Ni}(\mathrm{OTf})_{2}(10 \mathrm{~mol} \%)$ and degassed toluene solvent $(0.2 \mathrm{M})$ were added to the reaction mixture inside the glove box. Then the sealed tube was taken out of the glove box and reaction mixture was vigorously stirred at $140{ }^{\circ} \mathrm{C}$ on preheated aluminum block for $24 \mathrm{~h}$. After $24 \mathrm{~h}$ 
(upon completion of reaction as monitored by TLC analysis), the reaction mixture was cooled to room temperature and diluted with EtOAc and passed through a short celite pad. The solvent was evaporated under reduced pressure and the crude mixture was submitted for LCMS in methanol from which formation of stilbene was detected along with the formation of product 3ba.

LCMS (ESI) m/z of stilbene: $[\mathrm{M}+\mathrm{H}]^{+}$Calcd for $\mathrm{C}_{14} \mathrm{H}_{12}$ : 181.1; Found: 181.1.

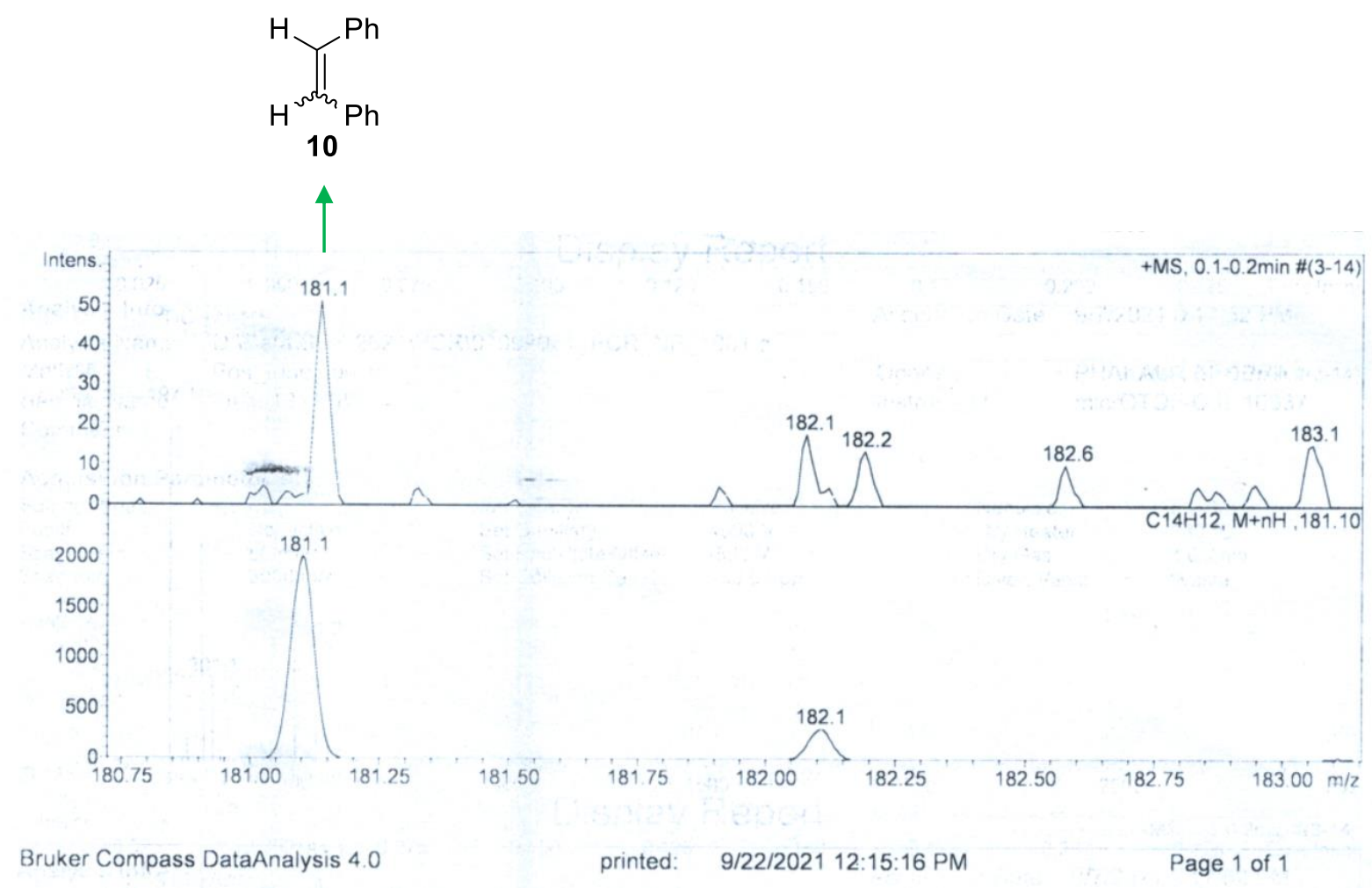

\subsection{Detection of deuterated-Stilbene in the reaction:}

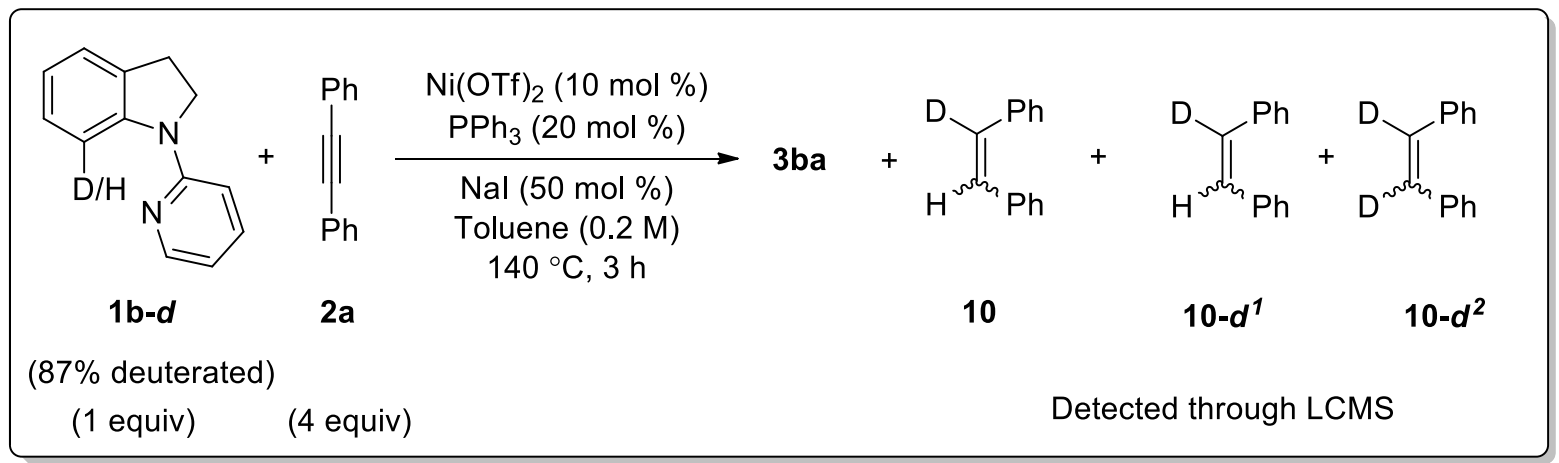

To an oven dried sealed tube charged with a stirring bar under $\mathrm{N}_{2}$ atmosphere, $\mathbf{1 b}-\boldsymbol{d}(10 \mathrm{mg}$, $0.05 \mathrm{mmol}$ ), diphenylacetylene $2 \mathbf{a}(36 \mathrm{mg}, 0.20 \mathrm{mmol}), \mathrm{PPh}_{3}(20 \mathrm{~mol} \%), \mathrm{NaI}(50 \mathrm{~mol} \%)$ were added and sealed. The sealed tube was high-vacuumed and refilled with $\mathrm{N}_{2}$. Then $\mathrm{Ni}(\mathrm{OTf})_{2}(10$ mol \%) and degassed toluene solvent $(0.2 \mathrm{M})$ were added to the reaction mixture inside the 
glove box. Then the sealed tube was taken out of the glove box and reaction mixture was vigorously stirred at $140{ }^{\circ} \mathrm{C}$ on preheated aluminum block for $3 \mathrm{~h}$. After $3 \mathrm{~h}$, the reaction mixture was cooled to room temperature and diluted with EtOAc and passed through a short celite pad. The solvent was evaporated under reduced pressure and the residue was submitted for LCMS in methanol from which formation of deuterated stilbenes $\left(\mathbf{1 0}, \mathbf{1 0}-\boldsymbol{d}^{\mathbf{1}}, \mathbf{1 0}-\boldsymbol{d}^{\mathbf{2}}\right)$ was detected along with the formation of product $3 \mathbf{b a}$.

(a) LCMS (ESI) m/z of 10: [M+H] ${ }^{+}$Calcd for $\mathrm{C}_{14} \mathrm{H}_{12}$ : 181.1; Found: 181.1 .

(b) LCMS (ESI) m/z of 10-d $:[\mathrm{M}+\mathrm{H}]^{+}$Calcd for $\mathrm{C}_{14} \mathrm{H}_{11} \mathrm{D}$ : 182.1 ; Found: 182.1 .

(c) LCMS (ESI) m/z of 10- $\boldsymbol{d}^{2}:[\mathrm{M}+\mathrm{H}]^{+}$Calcd for $\mathrm{C}_{14} \mathrm{H}_{10} \mathrm{D}_{2}$ : 183.1; Found: 183.1 .<smiles>C(=C/c1ccccc1)\c1ccccc1</smiles>

10

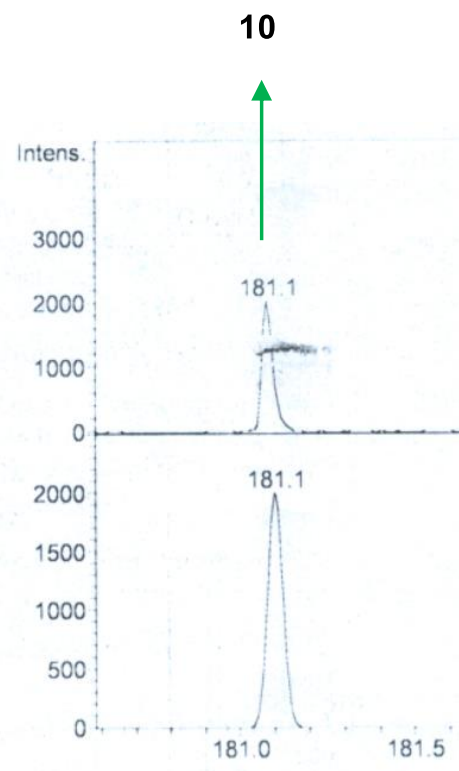

Bruker Compass DataAnalysis 4.0<smiles>[O+]C=Cc1ccccc1</smiles><smiles>[2H]/C(=C(/[2H])c1ccccc1)c1ccccc1</smiles>

$10-d^{1}$

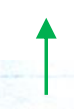

$10-d^{2}$
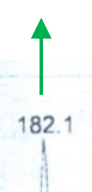

183.1 +MS, 0.0-0.1min \#(2-6)

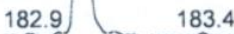
183.4
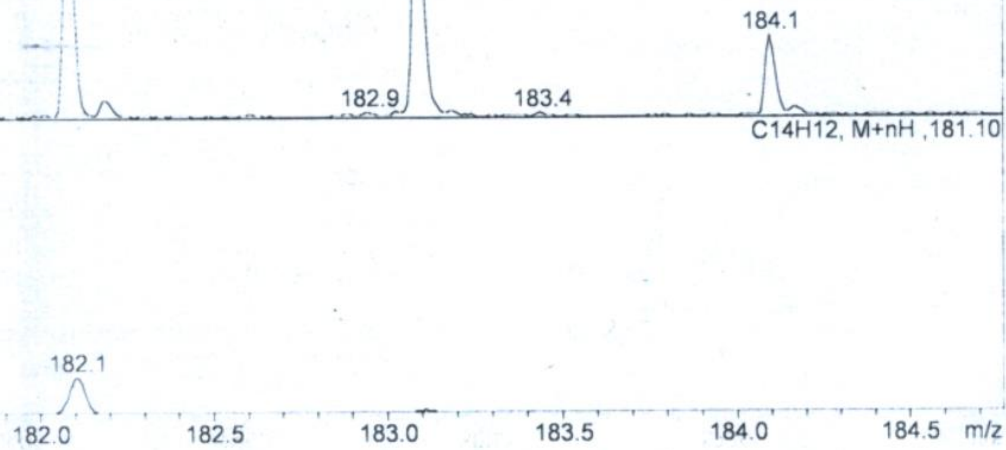

printed: $9 / 22 / 202112: 16: 12 \mathrm{PM}$

\section{Control experiments:}

\subsection{Synthesis of 3aa in presence of BHT:}

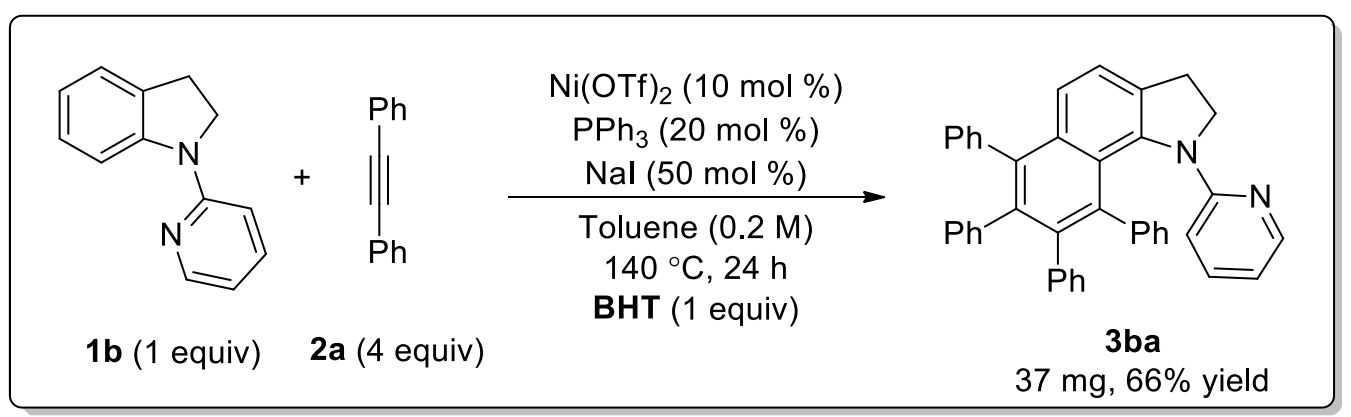


To an oven dried sealed tube charged with a stirring bar under $\mathrm{N}_{2}$ atmosphere, the $N$-pyridyl indoline $\mathbf{1 b}$ ( 1 equiv, $0.1 \mathrm{mmol}$ ), diphenylacetylene $\mathbf{2 a}$ (4 equiv, $0.4 \mathrm{mmol}), \mathrm{PPh}_{3}(20 \mathrm{~mol} \%)$, $\mathrm{NaI}$ (50 mol \%), BHT (1 equiv) were added and sealed. The sealed tube was high-vacuumed and refilled with $\mathrm{N}_{2}$. Then $\mathrm{Ni}(\mathrm{OTf})_{2}(10 \mathrm{~mol} \%)$ and degassed toluene solvent $(0.2 \mathrm{M})$ were added to the reaction mixture inside the glove box. Then the sealed tube was taken out of the glove box and reaction mixture was vigorously stirred at $140{ }^{\circ} \mathrm{C}$ on preheated aluminum block for $24 \mathrm{~h}$. After $24 \mathrm{~h}$ (upon completion of reaction as monitored by TLC analysis), the reaction mixture was cooled to room temperature and diluted with EtOAc and passed through a short celite pad. The solvent was evaporated under reduced pressure and the residue was purified by column chromatography using EtOAc/hexane mixture on silica gel to give the pure product $3 \mathbf{b a}(37 \mathrm{mg}, 66 \%$ yield).

\subsection{Synthesis of 3aa in presence of TEMPO:}

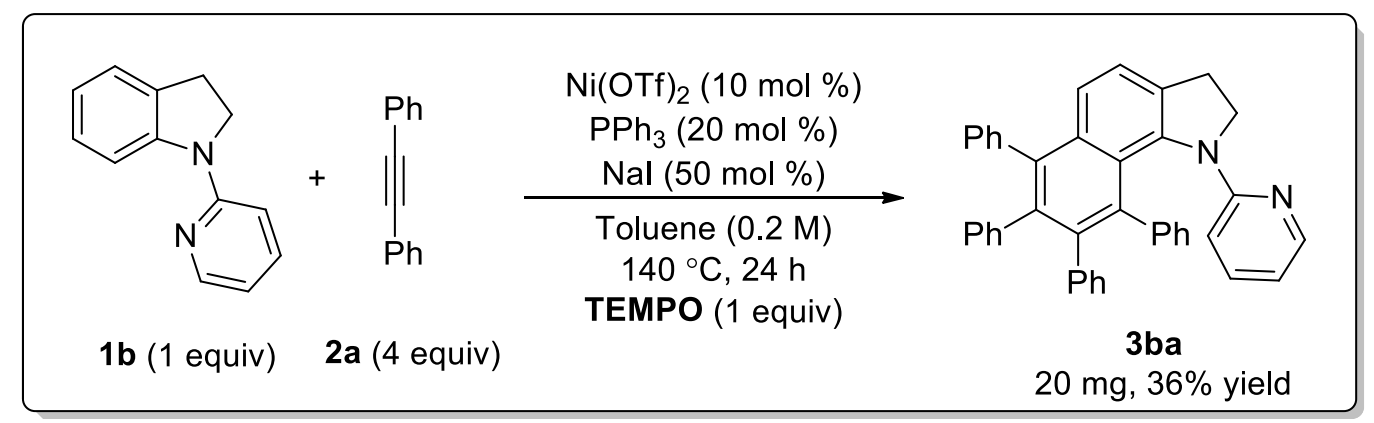

To an oven dried sealed tube charged with a stirring bar under $\mathrm{N}_{2}$ atmosphere, the $N$-pyridyl indoline $\mathbf{1 b}$ ( 1 equiv, $0.1 \mathrm{mmol}$ ), diphenylacetylene $\mathbf{2 a}$ (4 equiv, $0.4 \mathrm{mmol}), \mathrm{PPh}_{3}(20 \mathrm{~mol} \%)$, $\mathrm{NaI}$ (50 mol \%), TEMPO (1 equiv) were added and sealed. The sealed tube was high-vacuumed and refilled with $\mathrm{N}_{2}$. Then $\mathrm{Ni}(\mathrm{OTf})_{2}(10 \mathrm{~mol} \%)$ and degassed toluene solvent $(0.2 \mathrm{M})$ were added to the reaction mixture inside the glove box. Then the sealed tube was taken out of the glove box and reaction mixture was vigorously stirred at $140{ }^{\circ} \mathrm{C}$ on preheated aluminum block for $24 \mathrm{~h}$. After $24 \mathrm{~h}$ (upon completion of reaction as monitored by TLC analysis), the reaction mixture was cooled to room temperature and diluted with EtOAc and passed through a short celite pad. The solvent was evaporated under reduced pressure and the residue was purified by column chromatography using EtOAc/hexane mixture on silica gel to give the pure product 3ba (20 mg, 36\% yield). 


\section{Catalytic cycle:}

(a) Cascade C-H functionalization of $N$-pyridyl indoline:

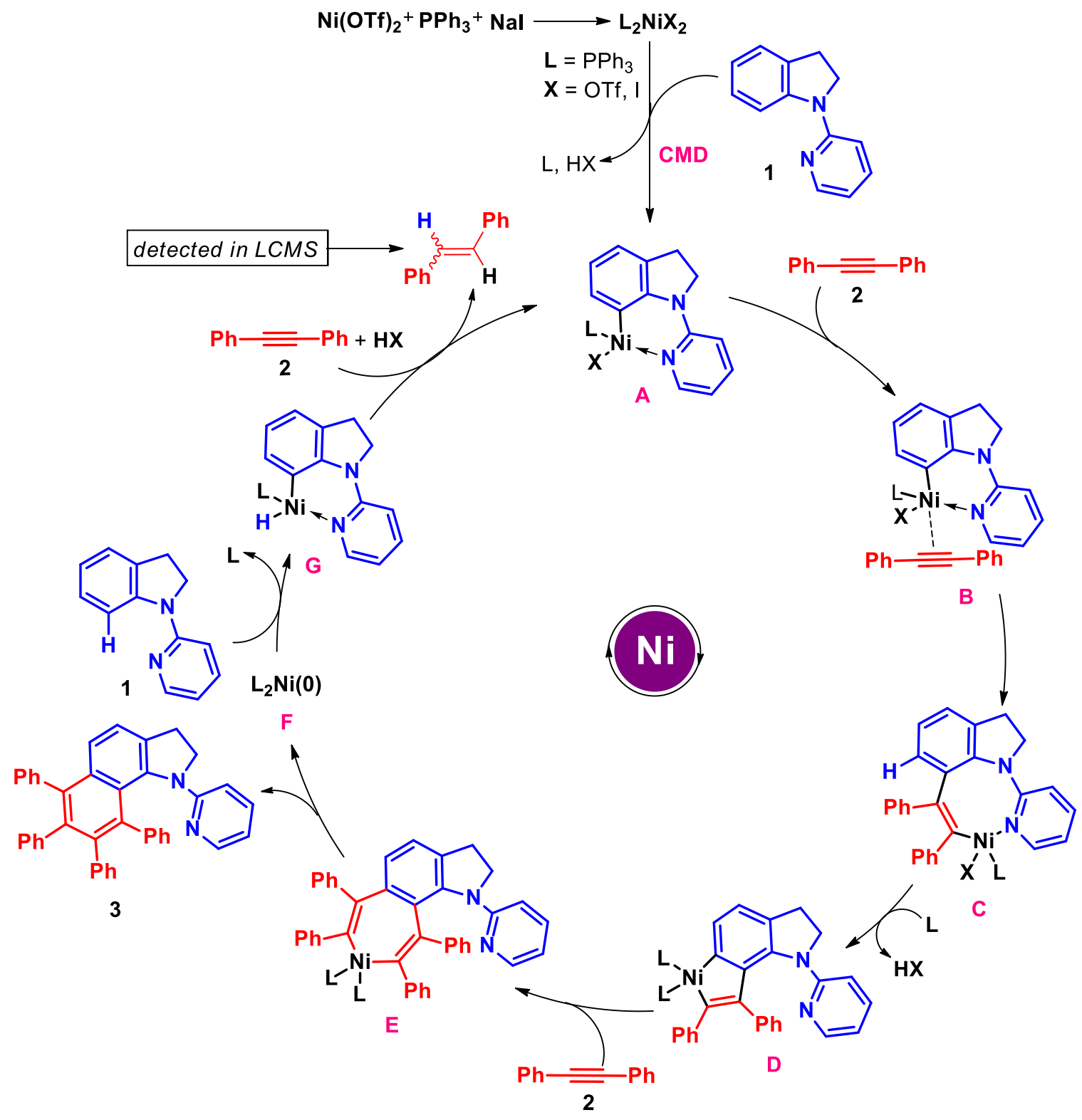

On the basis of above mechanistic studies and literature findings, ${ }^{6}$ a plausible catalytic cycle for the synthesis of $\mathbf{3}$ was depicted in scheme 5. Initially, the Ni(II)-catalyst undergoes ligand exchange to generate the active $\mathrm{Ni}(\mathrm{II})$-catalyst. Then indoline $\mathbf{1}$ coordinates with active $\mathrm{Ni}(\mathrm{II})$ catalyst to give intermediate $\mathbf{A}$ through concerted metallation-deprotonation (CMD) pathway. Then, alkyne $\mathbf{2}$ coordinates with intermediate $\mathbf{A}$ and gives intermediate $\mathbf{B}$. Migratory insertion of alkyne forms the intermediate $\mathbf{C}$. Then, second $\mathrm{C}-\mathrm{H}$ bond activation leads to the formation of five-membered cyclic intermediate D. Further, alkyne insertion followed by reductive eliminations delivers the desired product 3 along with the formation of $\mathrm{L}_{2} \mathrm{Ni}(0)$ intermediate $\mathbf{F}$. Then, the oxidative addition of $\mathrm{L}_{2} \mathrm{Ni}(0) \mathbf{F}$ species into $\mathrm{C}-\mathrm{H}$ bond of substrate $\mathbf{1}$ gives 
intermediate G. Reduction of alkyne (stilbene detected in HRMS) and protodemetallation regenerates active-intermediate $\mathbf{A}$ for the next catalytic cycle.

(b) Cascade C-H functionalization of N-pyridyl indole:

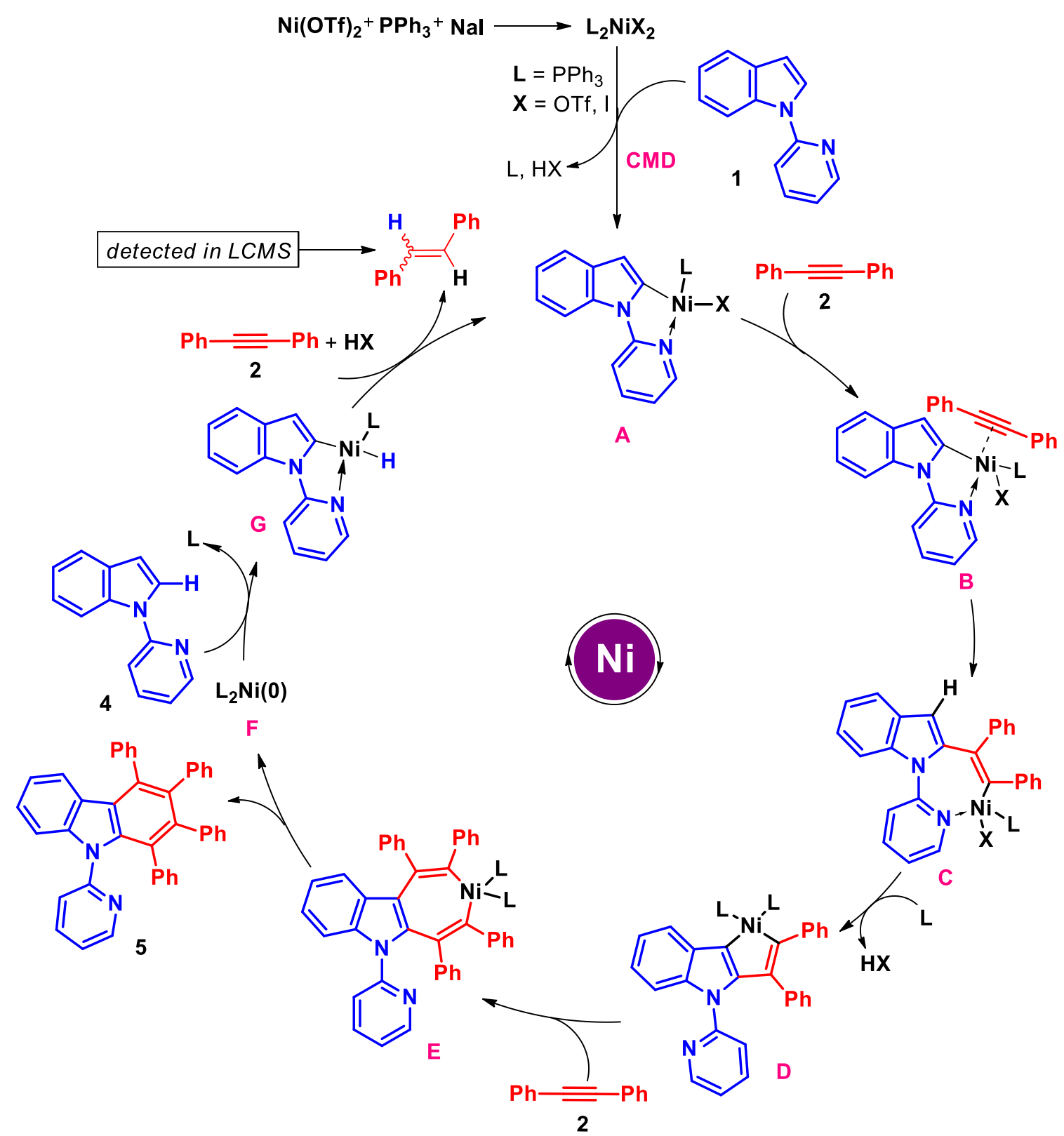

Similar to the above mechanism for the synthesis of carbazole product $\mathbf{5}$, initially indole $\mathbf{4}$ coordinates with $\mathrm{Ni}(\mathrm{II})$-catalyst to give intermediate $\mathbf{A}$ through concerted metallationdeprotonation (CMD) pathway. Then, alkyne $\mathbf{2}$ coordinates with intermediate $\mathbf{A}$ and gives intermediate B. Migratory insertion of alkyne forms the intermediate C. Then, second C-H bond activation leads to the formation of five-membered cyclic intermediate D. Further, alkyne insertion followed by reductive eliminations delivers the desired product $\mathbf{5}$ along with the formation of $\mathrm{L}_{2} \mathrm{Ni}(0)$ intermediate $\mathbf{F}$. Then, the oxidative addition of $\mathrm{L}_{2} \mathrm{Ni}(0) \mathbf{F}$ species into $\mathrm{C}$ - 
$\mathrm{H}$ bond of substrate $\mathbf{4}$ gives intermediate $\mathbf{G}$. Reduction of alkyne (stilbene detected in HRMS) and protodemetallation regenerates active-intermediate $\mathbf{A}$ for the next catalytic cycle.

\section{Synthetic applications:}

\section{1 $1 \mathrm{mmol}$ scale reaction for the synthesis of $3 \mathrm{ba}$ :}

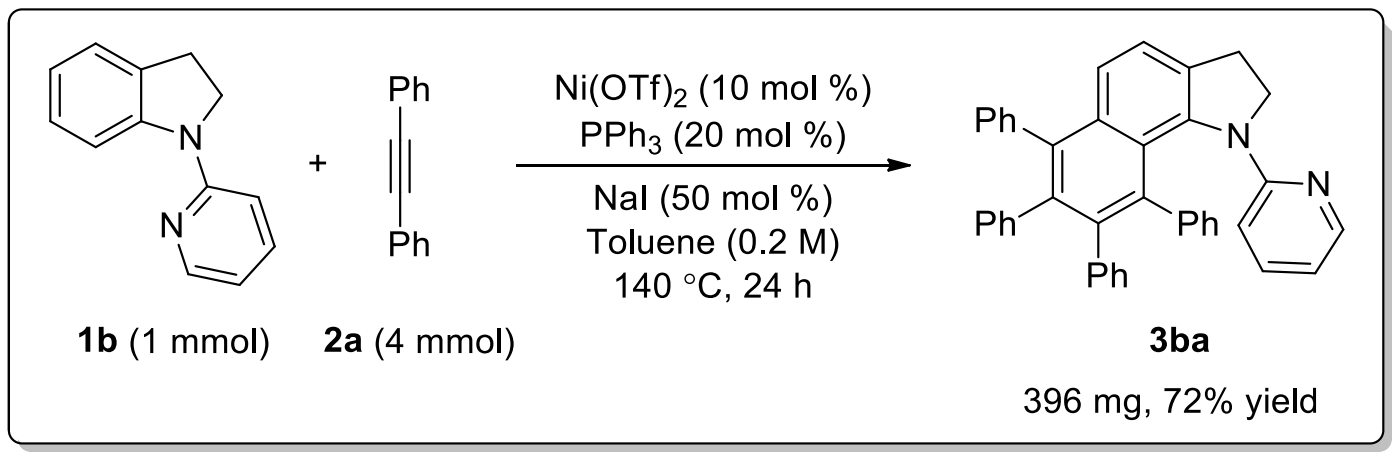

To an oven dried sealed tube charged with a stirring bar under $\mathrm{N}_{2}$ atmosphere, the $N$-pyridyl indoline $1 \mathbf{b}$ (196 mg, $1 \mathrm{mmol}$ ), diphenylacetylene 2a (713 mg, 4 mmol), $\mathrm{PPh}_{3}$ (53 mg, $20 \mathrm{~mol}$ $\%), \mathrm{NaI}$ (75 mg, $50 \mathrm{~mol} \%$ ) were added and sealed. The sealed tube was high-vacuumed and refilled with $\mathrm{N}_{2}$. Then $\mathrm{Ni}(\mathrm{OTf})_{2}(36 \mathrm{mg}, 10 \mathrm{~mol} \%)$ and degassed toluene solvent $(0.2 \mathrm{M}, 5$ $\mathrm{mL}$ ) were added to the reaction mixture inside the glove box. Then the sealed tube was taken out of the glove box and reaction mixture was vigorously stirred at $140{ }^{\circ} \mathrm{C}$ on preheated aluminum block for $24 \mathrm{~h}$. After $24 \mathrm{~h}$ (upon completion of reaction as monitored by TLC analysis), the reaction mixture was cooled to room temperature and diluted with EtOAc and passed through a short celite pad. The solvent was evaporated under reduced pressure and the residue was purified by column chromatography using 30\% EtOAc/hexane mixture on silica gel to give the pure product $3 \mathbf{b a}$ (396 $\mathrm{mg}$, $72 \%$ yield).

\section{2 $1 \mathrm{mmol}$ scale reaction for the synthesis of $5 \mathrm{ba}$ :}

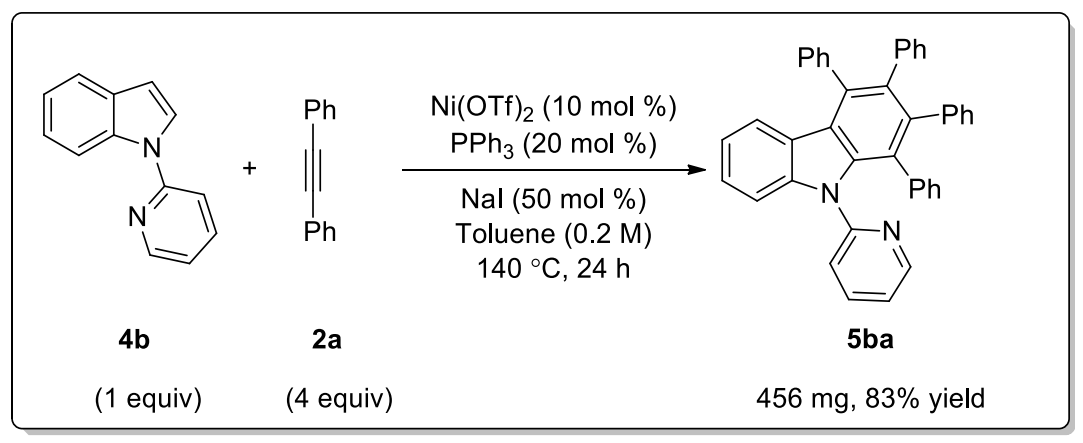

To an oven dried sealed tube charged with a stirring bar under $\mathrm{N}_{2}$ atmosphere, the $N$-pyridyl indole 4b (195 mg, 1 mmol), diphenylacetylene 2a (712 mg, 4 equiv), $\mathrm{PPh}_{3}$ (53 mg, $20 \mathrm{~mol}$ 
$\%), \mathrm{NaI}$ (75 mg, $50 \mathrm{~mol} \%$ ) were added and sealed. The sealed tube was high-vacuumed and refilled with $\mathrm{N}_{2}$. Then $\mathrm{Ni}(\mathrm{OTf})_{2}(36 \mathrm{mg}, 10 \mathrm{~mol} \%)$ and degassed toluene solvent $(0.2 \mathrm{M}, 5$ $\mathrm{mL}$ ) were added to the reaction mixture inside the glove box. Then the sealed tube was taken out of the glove box and reaction mixture was vigorously stirred at $140{ }^{\circ} \mathrm{C}$ on preheated aluminum block for $24 \mathrm{~h}$. After $24 \mathrm{~h}$ (completion of reaction as monitored by TLC analysis), the reaction mixture was cooled to room temperature and diluted with EtOAc and passed through a short celite pad. The solvent was evaporated under reduced pressure and the residue was purified by column chromatography using 25\% EtOAc/hexane mixture on silica gel to give the pure product $\mathbf{5 b a}(456 \mathrm{mg}, 83 \%$ yield).

\subsection{DDQ oxidation of $3 \mathrm{ba}:^{7}$}

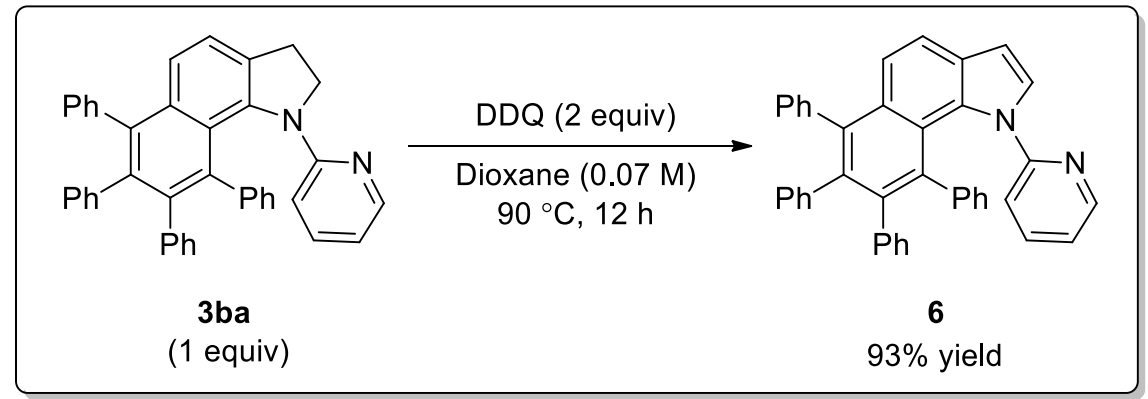

To an oven dried sealed tube charged with a stirring bar under $\mathrm{N}_{2}$ atmosphere, the compound 3ba (55 mg, 1 equiv, $0.1 \mathrm{mmol}$ ), DDQ (50 mg, 2 equiv, $0.22 \mathrm{mmol}$ ) were added followed by addition of 1,4-dioxane (0.07 M, $1.6 \mathrm{~mL})$ solvent. The reaction mixture was vigorously stirred at $90{ }^{\circ} \mathrm{C}$ on preheated aluminium block for the $12 \mathrm{~h}$. After $12 \mathrm{~h}$ (upon completion of reaction as monitored by TLC analysis), the reaction mixture was cooled to room temperature and diluted with EtOAc. The solvent was evaporated under reduced pressure and the residue was purified by column chromatography using EtOAc/hexane mixture on silica gel to give the pure product 6 (51 mg, 93\% yield).

\subsection{Directing group removal of $6:^{8}$}

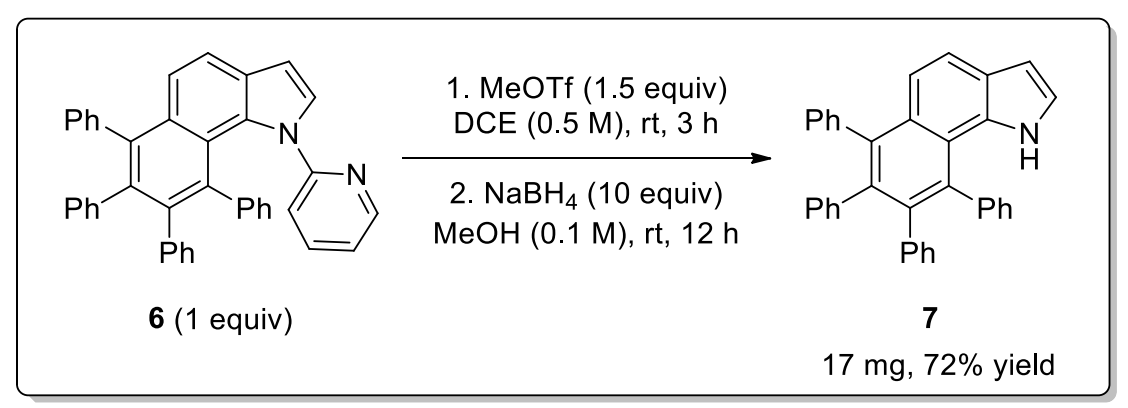

To an oven dried sealed tube charged with a stirring bar under $\mathrm{N}_{2}$ atmosphere, the compound 6 (26 mg, $0.05 \mathrm{mmol}, 1$ equiv) was added followed by addition of DCE (0.5 M, $0.1 \mathrm{~mL})$ solvent. The reaction mixture was cooled to $0{ }^{\circ} \mathrm{C}$ and then MeOTf ( $9 \mu \mathrm{L}, 0.07 \mathrm{mmol}, 1.5$ equiv) was 
added to the reaction mixture over 1 min under cold condition. Then the reaction mixture was stirred for $3 \mathrm{~h}$ at rt. After $3 \mathrm{~h}, \mathrm{MeOH}(0.1 \mathrm{M}, 0.5 \mathrm{~mL}), \mathrm{NaBH}_{4}(19 \mathrm{mg}, 0.5 \mathrm{mmol}, 10$ equiv) were added to the reaction mixture at $0{ }^{\circ} \mathrm{C}$, which was further stirred for $12 \mathrm{~h}$ at rt. The resulting mixture was quenched with saturated aqueous $\mathrm{NH}_{4} \mathrm{Cl}(2 \mathrm{~mL})$ solution and concentrated in vacuum. The aqueous layer was extracted with EtOAc $(5 \mathrm{~mL})$ and the organic layer was dried on anhydrous $\mathrm{MgSO}_{4}$ and concentrated in vacuum. The residue was purified by column chromatography using EtOAc/hexane mixture on silica gel to give the pure product 7 (17 mg, $72 \%$ yield).

\subsection{Directing group removal of $3 \mathrm{ba}:{ }^{8}$}

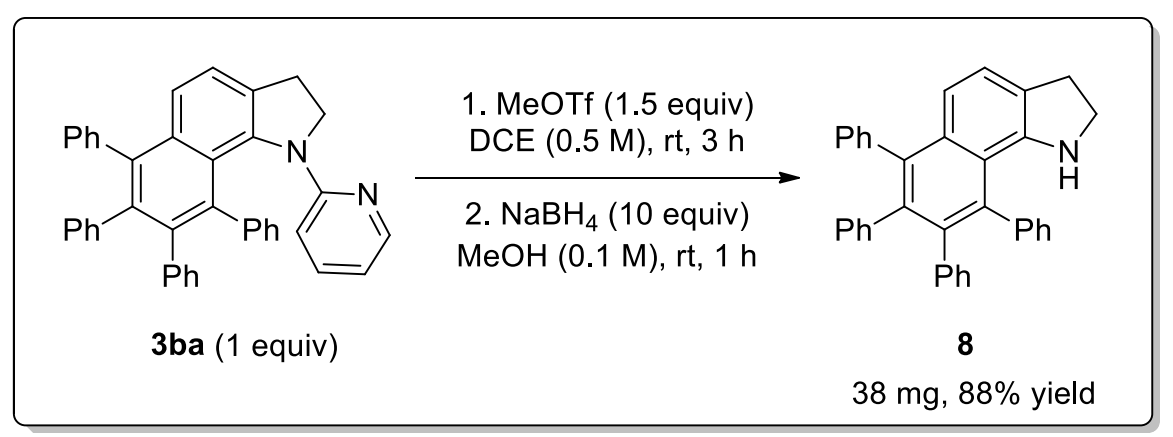

To an oven dried sealed tube charged with a stirring bar under $\mathrm{N}_{2}$ atmosphere, the compound 3ba (50 mg, $0.091 \mathrm{mmol}, 1$ equiv) was added followed by addition of DCE (0.5 M, $0.2 \mathrm{~mL})$ solvent. The reaction mixture was cooled to $0{ }^{\circ} \mathrm{C}$ and then MeOTf $(16 \mu \mathrm{L}, 0.14 \mathrm{mmol}, 1.5$ equiv) was added to the reaction mixture over 1 min under cold condition. Then the reaction mixture was stirred for $3 \mathrm{~h}$ at rt. After $3 \mathrm{~h}, \mathrm{MeOH}(0.1 \mathrm{M}, 0.91 \mathrm{~mL}), \mathrm{NaBH}_{4}(34 \mathrm{mg}, 0.91$ mmol, 10 equiv) were added to the reaction mixture at $0{ }^{\circ} \mathrm{C}$, which was further stirred for $1 \mathrm{~h}$ at $\mathrm{rt}$. The resulting mixture was quenched with saturated aqueous $\mathrm{NH}_{4} \mathrm{Cl}(2 \mathrm{~mL})$ solution and concentrated in vacuum. The aqueous layer was extracted with EtOAc $(5 \mathrm{~mL})$ and the organic layer was dried on anhydrous $\mathrm{MgSO}_{4}$ and concentrated in vacuum. The residue was purified by column chromatography using EtOAc/hexane mixture on silica gel to give the pure product 8 (38 mg, 88\% yield).

\subsection{Directing group removal of $5 \mathrm{ba}:^{9}$}

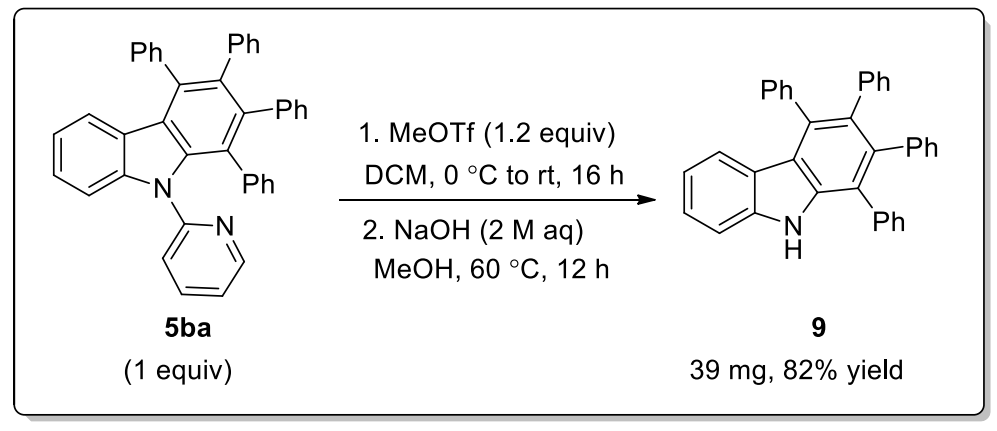


To an oven dried sealed tube charged with a stirring bar under $\mathrm{N}_{2}$ atmosphere, $\mathbf{5} \mathbf{b a}(55 \mathrm{mg}$, $0.10 \mathrm{mmol})$ was introduced followed by dichloromethane $(0.03 \mathrm{M}, 3.3 \mathrm{~mL})$ solvent, then MeOTf (14 $\mu \mathrm{L}, 0.12 \mathrm{mmol})$ was added drop wise to the reaction mixture at $0{ }^{\circ} \mathrm{C}$ and the resultant reaction mixture was stirred at room temperature for $16 \mathrm{~h}$. The volatiles were removed under vacuum and the residue was redissolved in $\mathrm{MeOH}(0.08 \mathrm{M}, 1.3 \mathrm{~mL})$. To the resultant mixture, $\mathrm{NaOH}(2 \mathrm{M}$ aq., $1.0 \mathrm{~mL}$ ) solution was added and the reaction mixture was stirred at $60{ }^{\circ} \mathrm{C}$ for $12 \mathrm{~h}$. after completion of reaction, the volatiles were evaporated under reduced pressure, and the resulting residue was extracted with EtOAc. The combined organic extract was washed with brine, dried over $\mathrm{Na}_{2} \mathrm{SO}_{4}$ and the volatiles were evaporated in vacuo. The residue was purified by column chromatography using EtOAc/hexane mixture on silica gel to give the pure product 9 (39 $\mathrm{mg}, 82 \%$ yield).

\subsection{Further annulation of compound 6:}

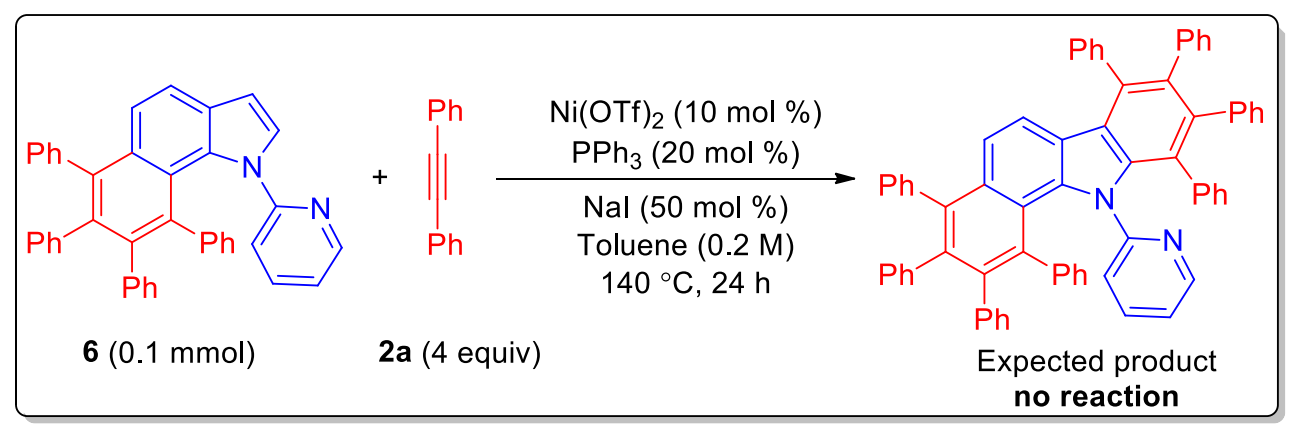

To an oven dried sealed tube charged with a stirring bar under $\mathrm{N}_{2}$ atmosphere, tetraphenyl-1(pyridin-2-yl)-1H-benzo[g]indole 6 (0.1 mmol), diphenylacetylene $\mathbf{2 a}$ (4 equiv), $\mathrm{PPh}_{3}$ (20 mol $\%), \mathrm{NaI}$ (50 mol \%), were added and sealed. The sealed tube was high-vacuumed and refilled with $\mathrm{N}_{2}$. Then $\mathrm{Ni}(\mathrm{OTf})_{2}(10 \mathrm{~mol} \%)$ and degassed toluene solvent $(0.2 \mathrm{M})$ were added to the reaction mixture inside the glove box. Then the sealed tube was taken out of the glove box and reaction mixture was vigorously stirred at $140{ }^{\circ} \mathrm{C}$ on preheated aluminum block for $24 \mathrm{~h}$. After $24 \mathrm{~h}$, we didn't observe formation of the product (even upon keeping for longer time and on higher temperature). For the further confirmation, the reaction mixture was cooled to room temperature and diluted with EtOAc or DCM and passed through a short celite pad. The solvent was evaporated under reduced pressure and the residue was purified by column chromatography using EtOAc/hexane mixture on silica gel, where we have recovered 92\% starting material 6.

\subsection{Unsuccessful coupling partners:}

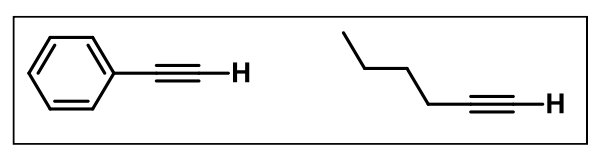




\section{References:}

1. Prusty, N.; Kinthada, L. K.; Meena, R.; Chebolu, R.; Ravikumar, P. C. Bismuth(III)-catalyzed regioselective alkylation of tetrahydroquinolines and indolines towards the synthesis of bioactive corebiaryl oxindoles and CYP19 inhibitors. Org. Biomol. Chem., 2021, 19, 891-905.

2. Gottlieb, H. E.; Kotlyar, V.; Nudelman, A. NMR chemical shifts of common laboratory solvents as trace impurities. J. Org. Chem.1997, 62, 7512-7515.

3. Gribble, G. W.; Hoffman, J. H. Reactions of sodium borohydride in acidic media. vi. reduction of indoles with cyanoborohydride in acetic acid. Synthesis, 1977, 859-860.

4. (a) Premi, C.; Dixit, A.; Jain, N. Palladium-Catalyzed Regioselective Decarboxylative Alkylation of Arenes and Heteroarenes with Aliphatic Carboxylic Acids. Org. Lett. 2015, 17, 2598-2601. (b) Lee, S. H.; Jeong, T.; Kim, K.; Kwon, N. Y.; Pandey, A. K.; Kim, H. S.; Ku, J.-M.; Mishra, N. K.; Kim, I. S. Ruthenium (II)-Catalyzed Site-Selective Hydroxymethylation of Indolines with Paraformaldehyde. $J$. Org. Chem. 2019, 84, 2307-2315. (c) Whyte, A.; Torelli, A.; Mirabi, B.; Prieto, L.; Rodriguez, J. F.; Lautens, M. Cobalt-Catalyzed Enantioselective Hydroarylation of 1, 6-Enynes. J. Am. Chem. Soc. 2020, 142, 9510-9517. (d) Zhao, X.; She, Y.; Fang, K.; Li, G. CuCl-catalyzed Ullmann-type C-N crosscoupling reaction of carbazoles and 2-bromopyridine derivatives. J. Org. Chem. 2017, 82, 1024- 1033.

5. Mio, M. J.; Kopel, L. C.; Braun, J. B.; Gadzikwa, T. L.; Hull, K. L.; Brisbois, R. G.; Markworth, C. J.; Grieco, P. A. One-pot synthesis of symmetrical and unsymmetrical bisarylethynes by a modification of the Sonogashira coupling reaction. Org. Lett. 2002, 4, 3199-3202.

6. (a) Zhao, T.; Pu, X.; Han, W.; Gao, G. Nickel-Catalyzed 3,3-Dialkynylation of 2-Aryl Acrylamides: Direct Access to gem-Diethynylethenes via Double Vinylic C-H Bond Activation. Org. Lett. 2021, 23, 4, 1199-1203. (b) Liu, Y.-H.; Xia, Y.-N.; Shi, B.-F. Ni-Catalyzed Chelation-Assisted Direct Functionalization of Inert C-H Bonds. Chin. J. Chem. 2020, 38, 635-662. (c) Guo, J.-R.; Gong, J.-F.; Song, M.-P. Nickel(ii)-catalyzed C(sp2)-H sulfuration/annulation with elemental sulfur: selective access to benzoisothiazolones. Org. Biomol. Chem. 2019, 17, 5029-5037. (d) Misal Castro, L. C.; Obata, A.; Aihara, Y.; Chatani, N. Chelation-Assisted Nickel-Catalyzed Oxidative Annulation via Double C-H Activation/Alkyne Insertion Reaction. Chem. Eur. J. 2016, 22, 1362-1367.

7. Ahmad, A.; Dutta, H. S.; Khan, B.; Kant, R.; Koley, D. Cu(I)-Catalyzed Site Selective Acyloxylation of Indoline Using $\mathrm{O}_{2}$ as the Sole Oxidant. Adv. Synth. Catal. 2018, 360, 1644-1649.

8. Lee, S. H.; Jeong, T.; Kim, K.; Kwon, N. Y.; Pandey, A. K.; Kim, H. S.; Ku, J.-M.; Mishra, N. K.; Kim, I. S. Ruthenium (II)-Catalyzed Site-Selective Hydroxymethylation of Indolines with Paraformaldehyde. J. Org. Chem. 2019, 84, 2307-2315.

9. (a) Tiwari, V. K.; Kamal, N.; Kapur, M. Ruthenium-Catalyzed Heteroatom-Directed Regioselective CH Arylation of Indoles Using a Removable Tether. Org. Lett. 2015, 17, 1766. (b) Soni, V.; Jagtap, R. A.; Gonnade, R. G.; Punji, B. Unified strategy for nickel-catalyzed C-2 alkylation of indoles through chelation assistance. ACS Catal., 2016, 6, 5666-5672. 


\section{8. (a) Experimental characterization data for products:}

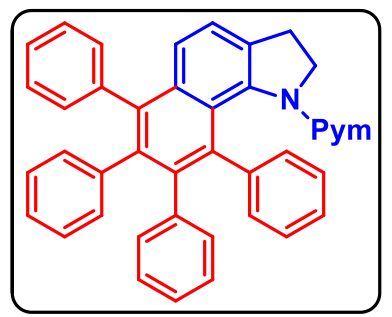

6,7,8,9-tetraphenyl-1-(pyrimidin-2-yl)-2,3-dihydro-1H-benzo[g]indole (3aa): was prepared according to general procedure (2.4). The crude reaction mixture was purified by column chromatography using silica gel (100-200 mesh size in 30\% EtOAc/hexane) giving 3aa (45 mg for 0.10 mmol scale) $82 \%$ yield.

Physical State: green solid

m.p.: $108-112^{\circ} \mathrm{C}$

$\mathbf{R}_{f}$-value: $0.3(10 \% \mathrm{EtOAc/hexane)}$

${ }^{1} \mathbf{H}$ NMR (CDCl 3,400 MHz): $\delta 8.01(\mathrm{br}, 2 \mathrm{H}), 7.48(\mathrm{~d}, J=7.6 \mathrm{~Hz}, 1 \mathrm{H}), 7.42-7.40(\mathrm{~m}, 1 \mathrm{H}), 7.35-$ $7.28(\mathrm{~m}, 2 \mathrm{H}), 7.18-7.09(\mathrm{~m}, 4 \mathrm{H}), 7.02-6.99(\mathrm{~m}, 2 \mathrm{H}), 6.93-6.77(\mathrm{~m}, 6 \mathrm{H}), 6.72(\mathrm{q}, J=6.8 \mathrm{~Hz}, 2 \mathrm{H})$, $6.57(\mathrm{~d}, J=7.2 \mathrm{~Hz}, 1 \mathrm{H}), 6.48(\mathrm{t}, J=4.8 \mathrm{~Hz}, 1 \mathrm{H}), 6.42(\mathrm{~d}, J=7.6 \mathrm{~Hz}, 1 \mathrm{H}), 6.33(\mathrm{t}, J=7.6 \mathrm{~Hz}, 1 \mathrm{H})$, $6.09(\mathrm{~d}, J=8.0 \mathrm{~Hz}, 1 \mathrm{H}), 4.53-4.49(\mathrm{~m}, 1 \mathrm{H}), 4.09-4.01(\mathrm{~m}, 1 \mathrm{H}), 3.40-3.31(\mathrm{~m}, 1 \mathrm{H}), 2.93-2.87(\mathrm{~m}$, $1 \mathrm{H})$.

${ }^{13} \mathbf{C}\left\{{ }^{1} \mathbf{H}\right\}$ NMR $\left(\mathbf{C D C l}_{3}, \mathbf{1 0 0} \mathbf{M H z}\right): \delta 161.7,157.6,156.8,141.2,140.9,140.7,140.1,139.7,139.3$, 138.9, 138.6, 136.3, 133.4, 133.1, 132.5, 132.1, 131.9, 131.7, 131.6 (2C), 131.5, 127.9, 127.5, 126.7 (2C), 126.6 (2C), 126.5, 125.9, 125.6, 125.4, 125.3, 124.2, 124.0, 122.7, 112.6, 53.7, 30.6.

IR $\left(\mathrm{KBr}, \mathrm{cm}^{-1}\right):$ 3439, 3053, 2984, 1601, 1264.

HRMS (ESI) m/z: [M+H] $]^{+}$Calcd for $\mathrm{C}_{40} \mathrm{H}_{30} \mathrm{~N}_{3}$ 552.2434; Found 552.2414.

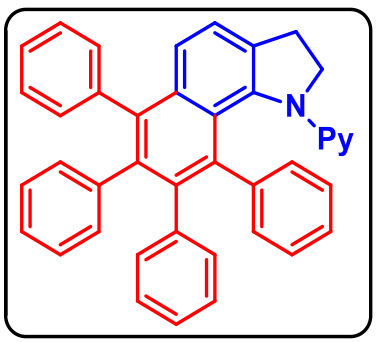

6,7,8,9-tetraphenyl-1-(pyridin-2-yl)-2,3-dihydro-1H-benzo[g]indole

(3ba): was prepared according to general procedure (2.4). The crude reaction mixture was purified by column chromatography using silica gel (100-200 mesh size in 20\% EtOAc/hexane) giving 3ba (51 mg for $0.10 \mathrm{mmol}$ scale) $93 \%$ yield.

Physical State: yellow solid

m.p.: $126-130{ }^{\circ} \mathrm{C}$

$\mathbf{R}_{f}$-value: 0.4 (10\% EtOAc/hexane)

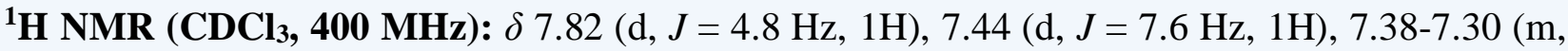
$3 \mathrm{H}), 7.19-7.11(\mathrm{~m}, 5 \mathrm{H}), 7.01(\mathrm{~d}, J=8.8 \mathrm{~Hz}, 2 \mathrm{H}), 6.92(\mathrm{~d}, J=7.6 \mathrm{~Hz}, 1 \mathrm{H}), 6.83-6.69(\mathrm{~m}, 7 \mathrm{H}), 6.58-$ $6.53(\mathrm{~m}, 2 \mathrm{H}), 6.42(\mathrm{~d}, J=7.6 \mathrm{~Hz}, 1 \mathrm{H}), 6.23(\mathrm{t}, J=7.6 \mathrm{~Hz}, 2 \mathrm{H}), 5.94(\mathrm{~d}, J=7.6 \mathrm{~Hz}, 1 \mathrm{H}), 4.32-4.27$ (m, 1H), 4.13-4.05 (m, 1H), 3.37-3.28 (m, 1H), 2.89 (q, $J=8.4 \mathrm{~Hz}, 1 \mathrm{H})$.

${ }^{13} \mathbf{C}\left\{{ }^{1} \mathbf{H}\right\}$ NMR $\left(\mathbf{C D C l}_{3}, \mathbf{1 0 0} \mathbf{M H z}\right): \delta 147.4,141.1,140.8,140.7,140.2,139.2,138.8,138.7,136.2$, 136.1, 136.0, 133.7, 133.4, 132.1, 131.9, 131.8, 131.7, 131.6, 131.5, 128.0, 127.6, 126.8, 126.7, $126.5,125.8,125.7,125.5,125.3,124.5,124.0,123.2,115.3,111.7,54.8,30.4$.

IR $\left(\mathrm{KBr}, \mathrm{cm}^{-1}\right): 3440,3053,2986,1652,1260$.

HRMS (ESI) m/z: $[\mathrm{M}+\mathrm{K}]^{+}$Calcd for $\mathrm{C}_{41} \mathrm{H}_{30} \mathrm{~N}_{2} \mathrm{~K}$ 589.2046; Found 589.2053. 


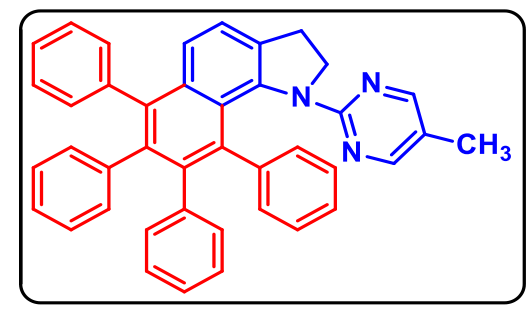

1-(5-methylpyrimidin-2-yl)-6,7,8,9-tetraphenyl-2,3-dihydro1H-benzo[g]indole (3ca): was prepared according to general procedure (2.4). The crude reaction mixture was purified by column chromatography using silica gel (100-200 mesh size in $30 \% \mathrm{EtOAc} / \mathrm{hexane})$ giving $3 \mathrm{ca}(17 \mathrm{mg}$ for $0.10 \mathrm{mmol} \mathrm{scale})$ $30 \%$ yield.

Physical State: yellow solid

m.p.: $115-120^{\circ} \mathrm{C}$

$\mathbf{R}_{\boldsymbol{f}}$-value: 0.3 (30\% EtOAc/hexane)

${ }^{1} \mathbf{H}$ NMR $\left(\mathbf{C D C l}_{3}, 400 \mathrm{MHz}\right): \delta 7.87(\mathrm{~s}, 2 \mathrm{H}), 7.48(\mathrm{~d}, J=7.6 \mathrm{~Hz}, 1 \mathrm{H}), 7.40-7.28(\mathrm{~m}, 3 \mathrm{H}), 7.18-$ $7.10(\mathrm{~m}, 4 \mathrm{H}), 7.02-6.99(\mathrm{~m}, 2 \mathrm{H}), 6.93-6.76(\mathrm{~m}, 6 \mathrm{H}), 6.75-6.69(\mathrm{~m}, 2 \mathrm{H}), 6.56(\mathrm{~d}, J=7.6 \mathrm{~Hz}, 1 \mathrm{H})$, $6.42(\mathrm{~d}, J=7.6 \mathrm{~Hz}, 1 \mathrm{H}), 6.28(\mathrm{t}, J=7.6 \mathrm{~Hz}, 1 \mathrm{H}), 6.06(\mathrm{~d}, J=7.6 \mathrm{~Hz}, 1 \mathrm{H}), 4.47-4.42(\mathrm{~m}, 1 \mathrm{H}), 4.10-$ $4.02(\mathrm{~m}, 1 \mathrm{H}), 3.40-3.31(\mathrm{~m}, 1 \mathrm{H}), 2.92-2.86(\mathrm{~m}, 1 \mathrm{H}), 2.07(\mathrm{~s}, 3 \mathrm{H})$.

${ }^{13} \mathbf{C}\left\{{ }^{1} \mathbf{H}\right\}$ NMR (CDCl 3,100 MHz): $\delta$ 160.7, 157.4, 156.9, 141.3, 141.0, 140.9, 140.8, 140.6 (2C), 139.6, 139.4, 139.0, 138.6, 136.4, 133.5, 133.0, 132.6, 132.2, 132.0, 131.9, 131.8, 131.7, 131.5, $127.9,127.5,126.9,126.7$ (2C), 126.6, 126.5, 126.4, 125.8, 125.6, 125.5, 125.4, 125.2, 125.15, $124.2,123.9,122.8,121.3,54.1,30.6,15.1$.

IR $\left(\mathrm{KBr}, \mathrm{cm}^{-1}\right):$ 3440, 3054, 2923, 2359, 1636, 1381.

HRMS (ESI) m/z: [M+H] $]^{+}$Calcd for $\mathrm{C}_{41} \mathrm{H}_{32} \mathrm{~N}_{3}$ 566.2591; Found 566.2567.

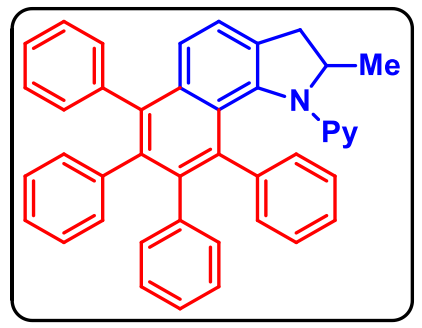

2-methyl-6,7,8,9-tetraphenyl-1-(pyridin-2-yl)-2,3-dihydro-1Hbenzo[g]indole (3fa): was prepared according to general procedure (2.4). The crude reaction mixture was purified by column chromatography using silica gel (100-200 mesh size in $20 \%$ EtOAc/hexane) giving $\mathbf{3 f a}$ (35 mg for $0.10 \mathrm{mmol} \mathrm{scale)} 62 \%$ yield.

Physical State: yellow solid

m.p.: $165-174{ }^{\circ} \mathrm{C}$

$\mathbf{R}_{\boldsymbol{f}}$-value: 0.2 (5\% EtOAc/hexane)

${ }^{1} \mathbf{H}$ NMR $\left(\mathbf{C D C l}_{3}, 400 \mathrm{MHz}\right): \delta 7.85\left(\mathrm{dd}, J_{l}=4.8 \mathrm{~Hz}, J_{2}=1.6 \mathrm{~Hz}, 1 \mathrm{H}\right), 7.46(\mathrm{~d}, J=7.6 \mathrm{~Hz}, 1 \mathrm{H})$, 7.40-7.30 (m, 3H), 7.19-7.07 (m, 5H), $7.01(\mathrm{t}, J=8.0 \mathrm{~Hz}, 2 \mathrm{H}), 6.92-6.89(\mathrm{~m}, 1 \mathrm{H}), 6.85-6.68(\mathrm{~m}$, $7 \mathrm{H}), 6.58-6.54(\mathrm{~m}, 2 \mathrm{H}), 6.42(\mathrm{~d}, J=7.6 \mathrm{~Hz}, 1 \mathrm{H}), 6.22(\mathrm{t}, J=7.6 \mathrm{~Hz}, 1 \mathrm{H}), 6.16(\mathrm{~d}, J=8.0 \mathrm{~Hz}, 1 \mathrm{H})$, $5.94(\mathrm{~d}, J=8.0 \mathrm{~Hz}, 1 \mathrm{H}), 4.51(\mathrm{br}, 1 \mathrm{H}), 3.49(\mathrm{q}, J=8.0 \mathrm{~Hz}, 1 \mathrm{H}), 2.52(\mathrm{~d}, J=15.6 \mathrm{~Hz}, 1 \mathrm{H}), 1.42(\mathrm{~d}$, $J=6.8 \mathrm{~Hz}, 3 \mathrm{H})$.

${ }^{13} \mathbf{C}\left\{{ }^{1} \mathbf{H}\right\}$ NMR $\left(\mathbf{C D C l}_{3}, \mathbf{1 0 0} \mathbf{M H z}\right): \delta 159.3,147.4,141.2,140.9,140.8,140.3,139.9,139.2,138.7$, 138.6, 136.3, 133.8, 133.3, 132.3, 132.2, 131.9, 131.7, 131.6 (2C), 131.5, 127.9, 127.5, 126.8, $126.6,126.4,125.9,125.5,125.4,125.2,124.6,123.94,123.9,123.7,115.7,111.5,63.3,37.6,22.1$.

IR $\left(\mathrm{KBr}, \mathrm{cm}^{-1}\right): 3440,2077,1636,1600,1264$.

HRMS (ESI) m/z: $[\mathrm{M}+\mathrm{H}]^{+}$Calcd for $\mathrm{C}_{42} \mathrm{H}_{33} \mathrm{~N}_{2}$ 565.2638; Found 565.2614. 


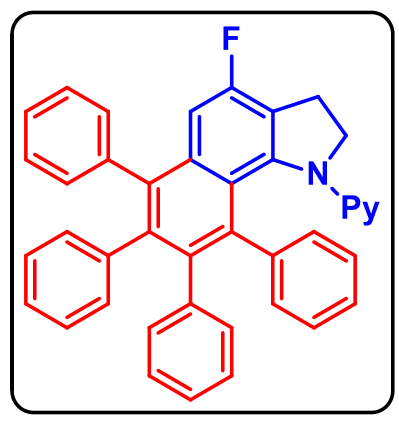

4-fluoro-6,7,8,9-tetraphenyl-1-(pyridin-2-yl)-2,3-dihydro-1Hbenzo[g]indole (3ga): was prepared according to general procedure (2.4). The crude reaction mixture was purified by column chromatography using silica gel (100-200 mesh size in $25 \%$ EtOAc/hexane) giving 3ga (48 $\mathrm{mg}$ for $0.10 \mathrm{mmol} \mathrm{scale}$ ) $84 \%$ yield.

Physical State: yellow solid

m.p.: $200-210{ }^{\circ} \mathrm{C}$

$\mathbf{R}_{\boldsymbol{f}}$-value: $0.3(20 \% \mathrm{EtOAc/hexane})$

${ }^{1} \mathbf{H}_{\text {NMR }}\left(\mathbf{C D C l}_{3}, 400 \mathrm{MHz}\right): \delta$ 7.86-7.84 (m, 1H), $7.43(\mathrm{~d}, J=7.6 \mathrm{~Hz}, 1 \mathrm{H}), 7.32\left(\mathrm{dt}, J_{l}=7.6 \mathrm{~Hz}\right.$, $\left.J_{2}=1.2 \mathrm{~Hz}, 1 \mathrm{H}\right), 7.20-7.11(\mathrm{~m}, 5 \mathrm{H}), 7.01-6.99(\mathrm{~m}, 3 \mathrm{H}), 6.91\left(\mathrm{dt}, J_{l}=7.6 \mathrm{~Hz}, J_{2}=1.2 \mathrm{~Hz}, 1 \mathrm{H}\right), 6.82-$ $6.69(\mathrm{~m}, 7 \mathrm{H}), 6.60-6.55(\mathrm{~m}, 2 \mathrm{H}), 6.40\left(\mathrm{dd}, J_{l}=7.6 \mathrm{~Hz}, J_{2}=1.2 \mathrm{~Hz}, 1 \mathrm{H}\right), 6.24-6.18(\mathrm{~m}, 2 \mathrm{H}), 5.91$ $(\mathrm{d}, J=8.0 \mathrm{~Hz}, 1 \mathrm{H}), 4.29-4.25(\mathrm{~m}, 1 \mathrm{H}), 4.19-4.11(\mathrm{~m}, 1 \mathrm{H}), 3.33-3.24(\mathrm{~m}, 1 \mathrm{H}), 3.03-2.96(\mathrm{~m}, 1 \mathrm{H})$.

${ }^{13} \mathbf{C}\left\{{ }^{1} \mathbf{H}\right\}$ NMR $\left(\mathbf{C D C l}_{3}, \mathbf{1 0 0} \mathbf{M H z}\right): \delta 158.9,158.0\left(\mathrm{~d}, J_{C-F}=243.3 \mathrm{~Hz}\right), 147.5,144.8\left(\mathrm{~d}, J_{C-F}=8.0\right.$ $\mathrm{Hz}), 141.0,140.6,140.3,139.7,139.4\left(\mathrm{~d}, J_{C-F}=2.0 \mathrm{~Hz}\right), 138.7(2 \mathrm{C}), 138.6,136.4(2 \mathrm{C}), 135.05(\mathrm{~d}$, $\left.J_{C-F}=8.0 \mathrm{~Hz}\right), 132.2,132.1,131.7(2 \mathrm{C}), 131.6,131.4(3 \mathrm{C}), 128.1,127.8,126.9,126.8,126.7,126.5$, $125.9,125.7,125.6,125.4,124.3,121.8\left(\mathrm{~d}, J_{C-F}=23.0 \mathrm{~Hz}\right), 120.4\left(\mathrm{~d}, J_{C-F}=1.0 \mathrm{~Hz}\right), 116.1,112.1$, $107.7\left(\mathrm{~d}, J_{C-F}=21.0 \mathrm{~Hz}\right), 55.4,26.7$.

${ }^{19} \mathrm{~F}$ NMR $\left(\mathrm{CDCl}_{3}, 376 \mathrm{MHz}\right): \delta-118.9$.

IR $\left(\mathrm{KBr}, \mathrm{cm}^{-1}\right): 3442,2099,1626,1264,1027,737$.

HRMS (ESI) m/z: $[\mathrm{M}+\mathrm{K}]^{+}$Calcd for $\mathrm{C}_{41} \mathrm{H}_{29} \mathrm{FN}_{2} \mathrm{~K}$ 607.1952; Found 607.1954.

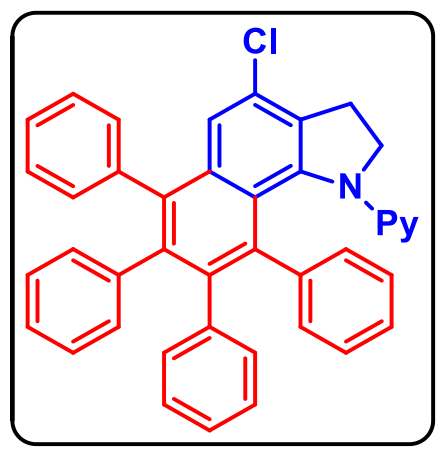

4-chloro-6,7,8,9-tetraphenyl-1-(pyridin-2-yl)-2,3-dihydro-1Hbenzo[g]indole (3ha): was prepared according to general procedure (2.4). The crude reaction mixture was purified by column chromatography using silica gel (100-200 mesh size in $40 \%$ EtOAc/hexane) giving 3ha (52 mg for $0.10 \mathrm{mmol} \mathrm{scale}$ ) $89 \%$ yield.

Physical State: yellow solid

m.p.: $175-180{ }^{\circ} \mathrm{C}$

$\mathbf{R}_{\boldsymbol{f}}$-value: $0.2(10 \% \mathrm{EtOAc/hexane})$

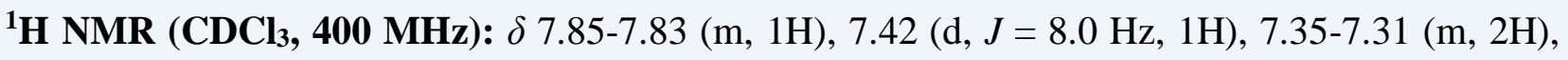
$7.18\left(\mathrm{tt}, J_{1}=7.6 \mathrm{~Hz}, J_{2}=1.2 \mathrm{~Hz}, 1 \mathrm{H}\right), 7.15-7.11(\mathrm{~m}, 4 \mathrm{H}), 7.00-6.97(\mathrm{~m}, 2 \mathrm{H}), 6.91(\mathrm{t}, J=7.6 \mathrm{~Hz}$, $1 \mathrm{H}), 6.82-6.68(\mathrm{~m}, 7 \mathrm{H}), 6.60-6.54(\mathrm{~m}, 2 \mathrm{H}), 6.40-6.38(\mathrm{~m}, 1 \mathrm{H}), 6.22(\mathrm{t}, J=7.6 \mathrm{~Hz}, 1 \mathrm{H}), 6.17(\mathrm{~d}, J$ $=8.4 \mathrm{~Hz}, 1 \mathrm{H}), 5.91-5.88(\mathrm{~m}, 1 \mathrm{H}), 4.25-4.10(\mathrm{~m}, 2 \mathrm{H}), 3.36-3.27(\mathrm{~m}, 1 \mathrm{H}), 3.05-2.98(\mathrm{~m}, 1 \mathrm{H})$.

${ }^{13} \mathbf{C}\left\{{ }^{1} \mathbf{H}\right\}$ NMR $\left(\mathbf{C D C l}_{3}, \mathbf{1 0 0} \mathbf{M H z}\right): \delta 158.8,147.4,143.1,140.9,140.5,140.3,140.0,139.7,138.6$, $138.5,136.5,136.3,134.8,132.0$ (2C), 131.8, 131.7, 131.6, 131.4, 129.4, 128.1, 127.8, 126.9, $126.8,126.7,126.5,125.9,125.8,125.6,125.4,124.3,122.7,121.7,116.0,112.0,54.6,29.9$.

IR $\left(\mathrm{KBr}, \mathrm{cm}^{-1}\right): 3441,3090,2376,1634,1323,700$.

HRMS (ESI) m/z: $[\mathrm{M}+\mathrm{H}]^{+}$Calcd for $\mathrm{C}_{41} \mathrm{H}_{30} \mathrm{ClN}_{2}$ 585.2092; Found 585.2108. 


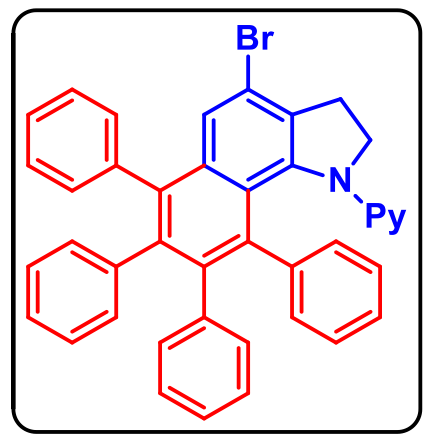

4-bromo-6,7,8,9-tetraphenyl-1-(pyridin-2-yl)-2,3-dihydro-1Hbenzo[g]indole (3ia): was prepared according to general procedure (2.4). The crude reaction mixture was purified by column chromatography using silica gel (100-200 mesh size in $30 \%$ EtOAc/hexane) giving 3ia (54 mg for $0.10 \mathrm{mmol} \mathrm{scale)} 86 \%$ yield.

Physical State: yellow solid

m.p.: $170-180{ }^{\circ} \mathrm{C} \quad \mathbf{R}_{\boldsymbol{f}}$-value: 0.3 (10\% EtOAc/hexane)

${ }^{1} \mathbf{H}$ NMR $\left(\mathbf{C D C l}_{3}, 400 \mathrm{MHz}\right): \delta 7.84(\mathrm{~d}, J=4.8 \mathrm{~Hz}, 1 \mathrm{H}), 7.50(\mathrm{~s}, 1 \mathrm{H}), 7.42(\mathrm{~d}, J=7.6 \mathrm{~Hz}, 1 \mathrm{H})$, $7.33(\mathrm{t}, J=7.6 \mathrm{~Hz}, 1 \mathrm{H}), 7.20-7.11(\mathrm{~m}, 5 \mathrm{H}), 6.99-6.96(\mathrm{~m}, 2 \mathrm{H}), 6.91(\mathrm{t}, J=7.6 \mathrm{~Hz}, 1 \mathrm{H}), 6.82-6.68$ $(\mathrm{m}, 7 \mathrm{H}), 6.59-6.53(\mathrm{~m}, 2 \mathrm{H}), 6.38(\mathrm{~d}, J=7.6 \mathrm{~Hz}, 1 \mathrm{H}), 6.22(\mathrm{t}, J=7.6 \mathrm{~Hz}, 1 \mathrm{H}), 6.16(\mathrm{~d}, J=8.0 \mathrm{~Hz}$, $1 \mathrm{H}), 5.88(\mathrm{~d}, J=8.0 \mathrm{~Hz}, 1 \mathrm{H}), 4.23-4.08(\mathrm{~m}, 2 \mathrm{H}), 3.36-3.26(\mathrm{~m}, 1 \mathrm{H}), 3.03-2.96(\mathrm{~m}, 1 \mathrm{H})$.

${ }^{13} \mathbf{C}\left\{{ }^{1} \mathbf{H}\right\}$ NMR $\left(\mathbf{C D C l}_{3}, \mathbf{1 7 6} \mathbf{M H z}\right): \delta 158.8,147.5,142.6,141.2,140.8,140.6,140.5,140.4,140.0$, 139.9, 139.7, 139.6, 138.6, 138.4, 138.2, 136.9, 136.5, 136.4 (2C), 136.3, 135.4, 135.1, 132.6, 132.0 (2C), 131.9 (2C), 131.8, 131.7, 131.6, 131.5 (2C), 131.4 (2C), 128.1 (2C), 127.8, 127.7, 126.9, 126.8, 126.7, 126.5, 125.9 (2C), 125.8, 125.6, 125.4, 124.3, 122.5, 122.0, 118.4, 116.0 (2C), 111.8, $111.7,91.7,35.6,31.9$.

IR $\left(\mathrm{KBr}, \mathrm{cm}^{-1}\right): 3439,3053,2928,1601,1264,895,738$.

HRMS (ESI) m/z: $[\mathrm{M}+\mathrm{H}]^{+}$Calcd for $\mathrm{C}_{41} \mathrm{H}_{30} \mathrm{BrN}_{2}$ 629.1587; Found 629.2319.

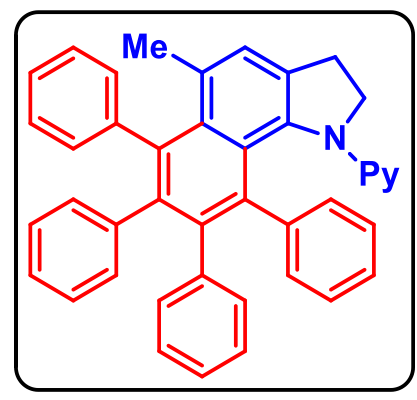

\begin{abstract}
5-methyl-6,7,8,9-tetraphenyl-1-(pyridin-2-yl)-2,3-dihydro-1Hbenzo[g]indole (3ja): was prepared according to general procedure (2.4). The crude reaction mixture was purified by column chromatography using silica gel (100-200 mesh size in $30 \%$ EtOAc/hexane) giving $3 \mathbf{j a}$ (44 $\mathrm{mg}$ for $0.10 \mathrm{mmol} \mathrm{scale}$ ) $78 \%$ yield.
\end{abstract}

Physical State: brown solid

m.p.: $140-142{ }^{\circ} \mathrm{C} \quad \mathbf{R}_{\boldsymbol{f}}$-value: 0.4 (10\% EtOAc/hexane)

${ }^{1} \mathrm{H}_{\mathrm{NMR}}\left(\mathrm{CDCl}_{3}, \mathbf{4 0 0} \mathrm{MHz}\right): \delta 7.83(\mathrm{~d}, J=4.0 \mathrm{~Hz}, 1 \mathrm{H}), 7.24-7.22(\mathrm{~m}, 2 \mathrm{H}), 7.20-7.07(\mathrm{~m}, 4 \mathrm{H})$, 7.02-6.96 (m, 2H), 6.88-6.70 (m, 10H), $6.60(\mathrm{~d}, J=7.6 \mathrm{~Hz}, 1 \mathrm{H}), 6.54(\mathrm{t}, J=6.0 \mathrm{~Hz}, 1 \mathrm{H}), 6.39(\mathrm{~d}$, $J=7.6 \mathrm{~Hz}, 1 \mathrm{H}), 6.25(\mathrm{t}, J=7.2 \mathrm{~Hz}, 2 \mathrm{H}), 5.91(\mathrm{~d}, J=8.0 \mathrm{~Hz}, 1 \mathrm{H}), 4.35(\mathrm{~d}, J=10.0 \mathrm{~Hz}, 1 \mathrm{H}), 4.03$ $(\mathrm{q}, J=11.2 \mathrm{~Hz}, 1 \mathrm{H}), 3.34-3.25(\mathrm{~m}, 1 \mathrm{H}), 2.91-2.85(\mathrm{~m}, 1 \mathrm{H}), 1.91(\mathrm{~s}, 3 \mathrm{H})$.

${ }^{13} \mathbf{C}\left\{{ }^{1} \mathbf{H}\right\}$ NMR $\left(\mathbf{C D C l}_{\mathbf{3}}, \mathbf{1 0 0} \mathbf{M H z}\right): \delta 147.4,143.3,141.2,140.8,139.9,139.6,139.3,139.1,138.6$, 136.2, 136.1, 132.8, 132.5, 132.3, 132.1, 132.0, 131.9, 131.7, 131.6, 131.5, 127.8, 127.2, 126.7, $126.6,126.5,126.4$ (2C), 125.9, 125.8, 125.2, 124.7, 124.3, 117.2, 114.8, 110.7, 53.9, 30.3, 25.6.

IR $\left(\mathrm{KBr}, \mathrm{cm}^{-1}\right):$ 3441, 3021, 2084, 1635, 1329, 1216.

HRMS (ESI) m/z: [M+Na $]^{+}$Calcd for $\mathrm{C}_{42} \mathrm{H}_{32} \mathrm{~N}_{2} \mathrm{Na}$ 587.2463; Found 587.2451. 


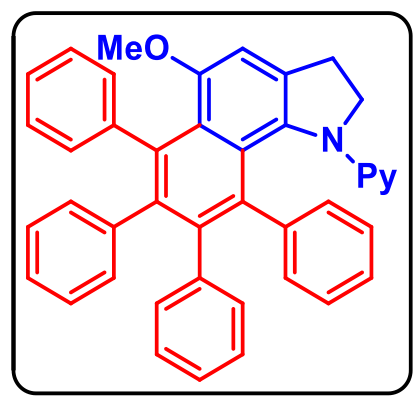

5-methoxy-6,7,8,9-tetraphenyl-1-(pyridin-2-yl)-2,3-dihydro-1Hbenzo[g]indole (3ka): was prepared according to general procedure (2.4). The crude reaction mixture was purified by column chromatography using silica gel (100-200 mesh size in $15 \%$ EtOAc/hexane) giving 3ka (55 $\mathrm{mg}$ for $0.10 \mathrm{mmol} \mathrm{scale}$ ) $95 \%$ yield.

Physical State: yellow liquid

$\mathbf{R}_{f}$-value: 0.5 (10\% EtOAc/hexane)

${ }^{1} \mathbf{H}$ NMR $\left(\mathbf{C D C l}_{3}, \mathbf{4 0 0} \mathbf{M H z}\right): \delta 7.82(\mathrm{~d}, J=4.0 \mathrm{~Hz}, 1 \mathrm{H}), 7.25-7.23(\mathrm{~m}, 1 \mathrm{H}), 7.18-7.10(\mathrm{~m}, 4 \mathrm{H})$, 7.02-6.95 (m, 2H), 6.93-6.89 (m, 2H), 6.86-6.68 (m, 9H), $6.56(\mathrm{~d}, J=7.2 \mathrm{~Hz}, 1 \mathrm{H}), 6.52(\mathrm{t}, J=6.0$ $\mathrm{Hz}, 1 \mathrm{H}), 6.39(\mathrm{~d}, J=6.0 \mathrm{~Hz}, 1 \mathrm{H}), 6.25-6.17(\mathrm{~m}, 2 \mathrm{H}), 5.93(\mathrm{~d}, J=8.0 \mathrm{~Hz}, 1 \mathrm{H}), 4.34(\mathrm{t}, J=8.6 \mathrm{~Hz}$, $1 \mathrm{H}), 4.03-3.95$ (m, 1H), 3.35 (s, 3H), 3.33-3.26 (m, 1H), 2.84 (q, $J=8.8 \mathrm{~Hz}, 1 \mathrm{H})$.

${ }^{13} \mathbf{C}\left\{{ }^{1} \mathbf{H}\right\}$ NMR $\left(\mathbf{C D C l}_{3}, \mathbf{1 0 0} \mathbf{M H z}\right): \delta 155.2,144.4,141.0,140.8,140.6,139.4,138.9,137.3,136.3$, 135.7, 133.7, 132.0, 131.9, 131.8, 131.4, 130.3, 129.9, 126.6 (2C), 126.4 (2C), 126.3, 125.8, 125.2 (2C), 125.1, 125.0, 124.4, 124.1, 114.7, 111.1, 106.0, 56.7, 54.3, 31.2.

IR $\left(\mathrm{KBr}, \mathrm{cm}^{-1}\right): 3445,3054,2365,1590,1382$.

HRMS (ESI) m/z: [M+Na] $]^{+}$Calcd for $\mathrm{C}_{42} \mathrm{H}_{32} \mathrm{~N}_{2} \mathrm{ONa}$ 603.2412; Found 603.2390.

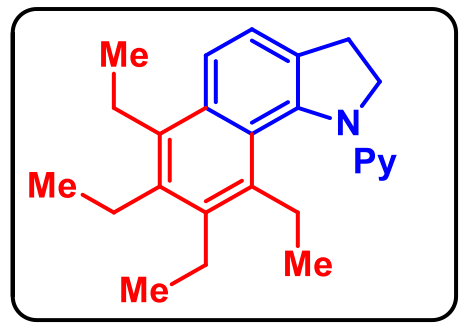

6,7,8,9-tetraethyl-1-(pyridin-2-yl)-2,3-dihydro-1H-benzo[g] indole (3bb): was prepared according to general procedure (2.4). The crude reaction mixture was purified by column chromatography using silica gel (100-200 mesh size in $15 \%$ EtOAc/hexane) giving $\mathbf{3 b b}(31 \mathrm{mg}$ for $0.10 \mathrm{mmol} \mathrm{scale}) 86 \%$ yield.

Physical State: colorless liquid

$\mathbf{R}_{\boldsymbol{f}}$-value: 0.5 (10\% EtOAc/hexane)

${ }^{1} \mathbf{H}$ NMR $\left(\mathbf{C D C l}_{\mathbf{3}}, \mathbf{4 0 0} \mathbf{M H z}\right): \delta 8.30(\mathrm{~d}, J=3.6 \mathrm{~Hz}, 1 \mathrm{H}), 7.85(\mathrm{~d}, J=8.4 \mathrm{~Hz}, 1 \mathrm{H}), 7.38(\mathrm{~d}, J=8.4$ $\mathrm{Hz}, 1 \mathrm{H}), 7.18-7.14(\mathrm{~m}, 1 \mathrm{H}), 6.67(\mathrm{t}, J=6.8 \mathrm{~Hz}, 1 \mathrm{H}), 6.06(\mathrm{~d}, J=8.4 \mathrm{~Hz}, 1 \mathrm{H}), 5.09-5.04(\mathrm{~m}, 1 \mathrm{H})$, 4.27-4.19 (m, 1H), 3.30-3.21 (m, 1H), 3.15-3.09 (m, 2H), 3.06-2.99 (m, 1H), 2.90-2.74 (m, 5H), 2.69-2.60 (m, 1H), $1.34(\mathrm{t}, J=7.6 \mathrm{~Hz}, 3 \mathrm{H}), 1.29(\mathrm{t}, J=7.6 \mathrm{~Hz}, 3 \mathrm{H}), 1.16(\mathrm{t}, J=7.6 \mathrm{~Hz}, 3 \mathrm{H}), 0.87$ (t, $J=7.6 \mathrm{~Hz}, 3 \mathrm{H})$.

${ }^{13} \mathbf{C}\left\{{ }^{1} \mathbf{H}\right\}$ NMR $\left(\mathbf{C D C l}_{\mathbf{3}}, \mathbf{1 0 0} \mathbf{M H z}\right): \delta 161.2,148.3,141.5,139.8,137.5,137.0,135.9,135.8,132.6$, $132.2,124.5,122.1,121.9,115.3,109.8,54.5,30.2,23.4,22.8,22.7,22.4,16.8,16.7,16.0$ (2C).

IR $\left(\mathrm{KBr}, \mathrm{cm}^{-1}\right): 3420,2964,1589,1375$.

HRMS (ESI) m/z: [M+H] $]^{+}$Calcd for $\mathrm{C}_{25} \mathrm{H}_{31} \mathrm{~N}_{2}$ 359.2482; Found 359.2487. 


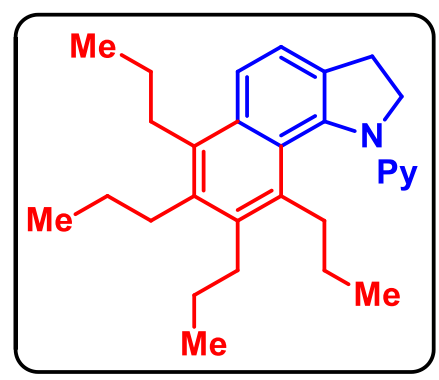

6,7,8,9-tetrapropyl-1-(pyridin-2-yl)-2,3-dihydro-1H-benzo[g] indole (3bc): was prepared according to general procedure (2.4). The crude reaction mixture was purified by column chromatography using silica gel (100-200 mesh size in $15 \%$ EtOAc/hexane) giving 3 bc (33 $\mathrm{mg}$ for $0.10 \mathrm{mmol} \mathrm{scale)} 80 \%$ yield.

Physical State: yellow liquid

$\mathbf{R}_{\boldsymbol{f}}$-value: 0.6 (10\% EtOAc/hexane)

${ }^{1} \mathbf{H}$ NMR $\left(\mathbf{C D C l}_{3}, \mathbf{4 0 0} \mathbf{M H z}\right): \delta 8.30(\mathrm{~d}, J=4.4 \mathrm{~Hz}, 1 \mathrm{H}), 7.79(\mathrm{~d}, J=8.4 \mathrm{~Hz}, 1 \mathrm{H}), 7.35(\mathrm{~d}, J=8.4$ $\mathrm{Hz}, 1 \mathrm{H}), 7.18-7.14(\mathrm{~m}, 1 \mathrm{H}), 6.67(\mathrm{t}, J=6.0 \mathrm{~Hz}, 1 \mathrm{H}), 6.06(\mathrm{~d}, J=8.4 \mathrm{~Hz}, 1 \mathrm{H}), 5.10-5.05(\mathrm{~m}, 1 \mathrm{H})$, 4.23-4.15 (m, 1H), 3.30-3.21 (m, 1H), 3.07-2.92 (m, 3H), 2.89-2.83 (m, 1H), 2.77-2.64 (m, 4H), 2.58-2.50 (m, 1H), 1.76-1.60 (m, 4H), 1.57-1.44 (m, 2H), 1.40-1.20 (m, 2H), 1.15-1.09 (m, 6H), $1.01(\mathrm{t}, J=7.2 \mathrm{~Hz}, 3 \mathrm{H}), 0.74(\mathrm{t}, J=7.2 \mathrm{~Hz}, 3 \mathrm{H})$.

${ }^{13} \mathbf{C}\left\{{ }^{1} \mathbf{H}\right\}$ NMR $\left(\mathbf{C D C l}_{3}, \mathbf{1 0 0} \mathbf{M H z}\right): \delta 161.1,148.3,141.6,138.8,136.9,136.6,134.7,134.3,132.4$, $132.3,124.9,122.1,121.7,115.2,109.7,54.4,33.3,32.5,32.3,31.6,30.2,25.8,25.7,25.1$, 25.0, $15.4,15.3,15.2,14.7$.

IR $\left(\mathrm{KBr}, \mathrm{cm}^{-1}\right): 3441,2955,2928,1653,1589,1374$.

HRMS (ESI) m/z: $[\mathrm{M}+\mathrm{H}]^{+}$Calcd for $\mathrm{C}_{29} \mathrm{H}_{39} \mathrm{~N}_{2}$ 415.3108; Found 415.3115.

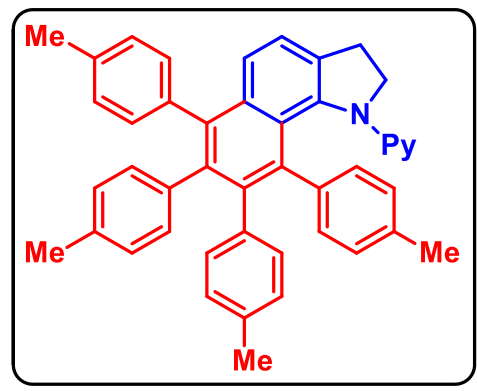

1-(pyridin-2-yl)-6,7,8,9-tetra-p-tolyl-2,3-dihydro-1H-benzo [g]indole (3bd): was prepared according to general procedure (2.4). The crude reaction mixture was purified by column chromatography using silica gel (100-200 mesh size in $25 \%$ EtOAc/hexane) giving $3 \mathrm{bd}$ (40 $\mathrm{mg}$ for $0.10 \mathrm{mmol} \mathrm{scale}$ ) $66 \%$ yield.

Physical State: pale white solid

m.p.: $264-266^{\circ} \mathrm{C}$

$\mathbf{R}_{f}$-value: 0.3 (10\% EtOAc/hexane)

${ }^{1} \mathbf{H}$ NMR $\left(\mathbf{C D C l}_{3}, \mathbf{4 0 0} \mathbf{M H z}\right): \delta 7.81(\mathrm{dd}, J=5.2 \mathrm{~Hz}, J=1.2 \mathrm{~Hz}, 1 \mathrm{H}), 7.31-7.25(\mathrm{~m}, 3 \mathrm{H}), 7.12-$ $7.05(\mathrm{~m}, 2 \mathrm{H}), 6.99(\mathrm{dd}, J=7.6 \mathrm{~Hz}, J=1.6 \mathrm{~Hz}, 1 \mathrm{H}), 6.94-6.85(\mathrm{~m}, 4 \mathrm{H}), 6.73-6.67(\mathrm{~m}, 2 \mathrm{H}), 6.62(\mathrm{~d}$, $J=8.0 \mathrm{~Hz}, 1 \mathrm{H}), 6.83-6.49(\mathrm{~m}, 3 \mathrm{H}), 6.43(\mathrm{~d}, J=7.6 \mathrm{~Hz}, 1 \mathrm{H}), 6.28(\mathrm{dd}, J=7.6 \mathrm{~Hz}, J=1.6 \mathrm{~Hz}, 1 \mathrm{H})$, $6.19(\mathrm{~d}, J=8.4 \mathrm{~Hz}, 1 \mathrm{H}), 6.00(\mathrm{~d}, J=7.2 \mathrm{~Hz}, 1 \mathrm{H}), 5.80(\mathrm{dd}, J=7.6 \mathrm{~Hz}, J=1.6 \mathrm{~Hz}, 1 \mathrm{H}), 429(\mathrm{t}, J=$ $10.0 \mathrm{~Hz}, 1 \mathrm{H}), 4.13-4.03(\mathrm{~m}, 1 \mathrm{H}), 3.35-3.25(\mathrm{~m}, 1 \mathrm{H}), 2.86(\mathrm{q}, J=8.4 \mathrm{~Hz}, 1 \mathrm{H}), 2.30(\mathrm{~s}, 3 \mathrm{H}), 2.11(\mathrm{~s}$, $3 \mathrm{H}), 2.09(\mathrm{~s}, 3 \mathrm{H}), 2.07$ (s, 3H).

${ }^{13} \mathbf{C}\left\{{ }^{1} \mathbf{H}\right\}$ NMR $\left(\mathbf{C D C l}_{3}, \mathbf{1 0 0} \mathbf{M H z}\right): \delta 139.1,138.9,138.2,137.8,135.8,135.6,134.5,134.4,134.0$, $133.8,133.0,132.2$, 131.9, 131.8, 131.5, 131.4, 131.3, 131.3, 131.2, 128.7, 128.2, 127.5, 127.4, $127.1,126.5,124.6,123.2,122.8,115.0,54.9,30.3,21.5,21.5,21.4,21.3$.

IR $\left(\mathrm{KBr}, \mathrm{cm}^{-1}\right): 3438,3053,2923,2351,1642,1434$.

HRMS (ESI) m/z: $[\mathrm{M}+\mathrm{H}]^{+}$Calcd for $\mathrm{C}_{45} \mathrm{H}_{39} \mathrm{~N}_{2}$ 607.3108; Found 607.3092. 


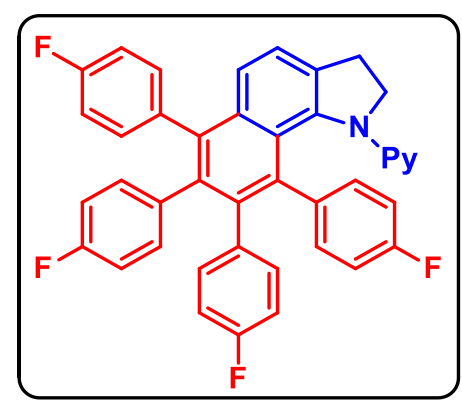

6,7,8,9-tetrakis(4-fluorophenyl)-1-(pyridin-2-yl)-2,3-dihydro1H-benzo[g]indole (3bf): was prepared according to general procedure (2.4). The crude reaction mixture was purified by column chromatography using silica gel (100-200 mesh size in $15 \%$ EtOAc/hexane) giving $3 \mathbf{b f}$ (48 $\mathrm{mg}$ for $0.10 \mathrm{mmol} \mathrm{scale}$ ) $77 \%$ yield.

Physical State: yellow solid

m.p.: $160-170{ }^{\circ} \mathrm{C}$

$\mathbf{R}_{f}$-value: 0.3 (5\% EtOAc/hexane)

${ }^{1} \mathbf{H}$ NMR $\left(\mathbf{C D C l}_{\mathbf{3}}, \mathbf{4 0 0} \mathbf{M H z}\right): \delta 7.87(\mathrm{~d}, J=4.4 \mathrm{~Hz}, 1 \mathrm{H}), 7.40-7.30(\mathrm{~m}, 3 \mathrm{H}), 7.17-7.01(\mathrm{~m}, 3 \mathrm{H})$, 6.93-6.82 (m, 4H), 6.75-6.72 (m, 1H), $6.66(\mathrm{t}, J=8.4 \mathrm{~Hz}, 1 \mathrm{H}), 6.61-6.45(\mathrm{~m}, 5 \mathrm{H}), 6.33(\mathrm{t}, J=6.4$ $\mathrm{Hz}, 1 \mathrm{H}), 6.18(\mathrm{~d}, J=8.4 \mathrm{~Hz}, 1 \mathrm{H}), 5.97-5.93(\mathrm{~m}, 1 \mathrm{H}), 5.88-5.85(\mathrm{~m}, 1 \mathrm{H}), 4.22-4.10(\mathrm{~m}, 2 \mathrm{H}), 3.41-$ $3.31(\mathrm{~m}, 1 \mathrm{H}), 2.94-2.88(\mathrm{~m}, 1 \mathrm{H})$.

$\left.{ }^{13} \mathbf{C}^{1}{ }^{1} \mathbf{H}\right\}$ NMR $\left(\mathbf{C D C l}_{3}, 100 \mathrm{MHz}\right): \delta 162.2,\left(\mathrm{~d}, J_{C-F}=121.4 \mathrm{~Hz}\right), 161.7\left(\mathrm{~d}, J_{C-F}=3.5 \mathrm{~Hz}\right), 160.8$ $\left(\mathrm{d}, J_{C-F}=121.4 \mathrm{~Hz}\right), 160.3\left(\mathrm{~d}, J_{C-F}=3.5 \mathrm{~Hz}\right), 147.1,141.3,141.2\left(\mathrm{~d}, J_{C-F}=1.8 \mathrm{~Hz}\right), 139.3,138.8$, $137.9,136.8\left(\mathrm{~d}, J_{C-F}=3.5 \mathrm{~Hz}\right), 136.7,136.4\left(\mathrm{~d}, J_{C-F}=3.5 \mathrm{~Hz}\right), 136.25\left(\mathrm{~d}, J_{C-F}=3.5 \mathrm{~Hz}\right), 135.6$, $134.5\left(\mathrm{~d}, J_{C-F}=1.8 \mathrm{~Hz}\right), 133.8,133.5,133.4,133.3,133.2(2 \mathrm{C}), 133.1,133.05,133.0\left(\mathrm{~d}, J_{C-F}=3.5\right.$ $\mathrm{Hz}), 132.9$ (2C), $132.8(3 \mathrm{C}), 131.9,128.6\left(\mathrm{~d}, J_{C-F}=14.1 \mathrm{~Hz}\right), 124.5,123.7,123.3,115.8,115.3$, $115.1,114.9,114.8,114.3,114.2,114.1,114.0,113.9,112.9\left(\mathrm{~d}, J_{C-F}=22.9 \mathrm{~Hz}\right), 112.1,111.2(\mathrm{~d}$, $\left.J_{C-F}=22.9 \mathrm{~Hz}\right), 55.4,30.4$.

${ }^{19}$ F NMR (CDCl 3,376 MHz): $\delta$-115.6, -116.7, -116.9.

IR $\left(\mathrm{KBr}, \mathrm{cm}^{-1}\right):$ 3430, 3045, 2926, 1602, 1264, 1157, 1094.

HRMS (ESI) m/z: $[\mathrm{M}+\mathrm{H}]^{+}$Calcd for $\mathrm{C}_{41} \mathrm{H}_{27} \mathrm{~F}_{4} \mathrm{~N}_{2}$ 623.2105; Found 623.2056.

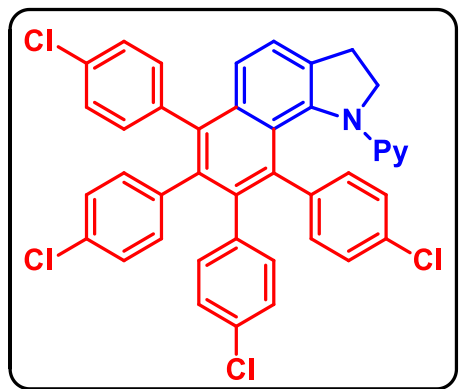

6,7,8,9-tetrakis(4-chlorophenyl)-1-(pyridin-2-yl)-2,3-dihydro-

1H-benzo[g]indole (3bg): was prepared according to general procedure (2.4). The crude reaction mixture was purified by column chromatography using silica gel (100-200 mesh size in $40 \%$ EtOAc/hexane) giving $3 \mathrm{bg}$ (42 $\mathrm{mg}$ for $0.10 \mathrm{mmol} \mathrm{scale}$ ) $61 \%$ yield.

Physical State: yellow solid

m.p.: $200-210^{\circ} \mathrm{C}$

$\mathbf{R}_{f}$-value: 0.4 (30\% EtOAc/hexane)

${ }^{1} \mathbf{H}$ NMR $\left(\mathbf{C D C l}_{3}, 400 \mathrm{MHz}\right): \delta 7.86(\mathrm{~d}, J=3.6 \mathrm{~Hz}, 1 \mathrm{H}), 7.39-7.31(\mathrm{~m}, 3 \mathrm{H}), 7.28-7.26(\mathrm{~m}, 2 \mathrm{H}), 7.16-$ $7.04(\mathrm{~m}, 4 \mathrm{H}), 6.97-6.94(\mathrm{~m}, 1 \mathrm{H}), 6.90-6.85(\mathrm{~m}, 2 \mathrm{H}), 6.81-6.70(\mathrm{~m}, 3 \mathrm{H}), 6.60\left(\mathrm{dd}, J_{1}=6.4 \mathrm{~Hz}, J_{2}=\right.$ $5.2 \mathrm{~Hz}, 1 \mathrm{H}), 6.46\left(\mathrm{dd}, J_{l}=8.4 \mathrm{~Hz}, J_{2}=2.0 \mathrm{~Hz}, 1 \mathrm{H}\right), 6.32\left(\mathrm{dd}, J_{l}=8.0 \mathrm{~Hz}, J_{2}=2.0 \mathrm{~Hz}, 1 \mathrm{H}\right), 6.21(\mathrm{dd}$, $\left.J_{l}=8.4 \mathrm{~Hz}, J_{2}=2.0 \mathrm{~Hz}, 1 \mathrm{H}\right), 6.11(\mathrm{~d}, J=8.4 \mathrm{~Hz}, 1 \mathrm{H}), 5.81\left(\mathrm{dd}, J_{l}=8.4 \mathrm{~Hz}, J_{2}=2.0 \mathrm{~Hz}, 1 \mathrm{H}\right), 4.16-$ $4.11(\mathrm{~m}, 2 \mathrm{H}), 3.41-3.32(\mathrm{~m}, 1 \mathrm{H}), 2.94-2.87(\mathrm{~m}, 1 \mathrm{H})$.

${ }^{13} \mathbf{C}\left\{{ }^{1} \mathbf{H}\right\}$ NMR $\left(\mathbf{C D C l}_{3}, \mathbf{1 0 0} \mathbf{M H z}\right): \delta 159.1,147.3,141.5,139.0,138.7,138.7,138.6,138.4,137.1$, 136.9, 136.5, 135.6, 133.8, 133.6, 133.2, 133.1, 133.0, 133.0, 132.9, 132.8, 132.6, 132.5, 132.0, 131.9, 128.6, 128.2, 127.6, 127.5, 127.4, 127.3, 126.0, 124.5, 124.3, 123.8, 123.2, 116.1, 112.3, 55.4, 30.4.

IR $\left(\mathrm{KBr}, \mathrm{cm}^{-1}\right): 3450,2364,2309,1635,1385,736$.

HRMS (ESI) m/z: $[\mathrm{M}+\mathrm{H}]^{+}$Calcd for $\mathrm{C}_{41} \mathrm{H}_{27} \mathrm{Cl}_{4} \mathrm{~N}_{2}$ 687.0923; Found 687.0896. 


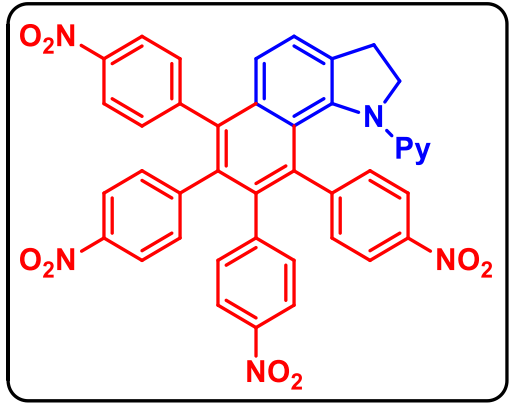

6,7,8,9-tetrakis(4-nitrophenyl)-1-(pyridin-2-yl)-2,3-dihydro 1H-benzo[g]indole (3bh): was prepared according to general procedure (2.4). The crude reaction mixture was purified by column chromatography using silica gel (100-200 mesh size in $50 \% \mathrm{EtOAc} / \mathrm{hexane})$ giving $\mathbf{3 b h}(54 \mathrm{mg}$ for $0.10 \mathrm{mmol} \mathrm{scale})$ $74 \%$ yield.

Physical State: yellow solid

m.p.: $295-300{ }^{\circ} \mathrm{C}$

$\mathbf{R}_{f}$-value: 0.35 (40\% EtOAc/hexane)

${ }^{1} \mathbf{H}_{\text {NMR }}\left(\mathbf{C D C l}_{\mathbf{3}}, \mathbf{4 0 0} \mathbf{M H z}\right): \delta 8.26(\mathrm{~d}, J=8.4 \mathrm{~Hz}, 1 \mathrm{H}), 8.08-8.02(\mathrm{~m}, 2 \mathrm{H}), 7.91-7.79(\mathrm{~m}, 3 \mathrm{H})$, 7.72-7.66 (m, 3H), $7.51(\mathrm{~d}, J=8.4 \mathrm{~Hz}, 1 \mathrm{H}), 7.38(\mathrm{~d}, J=8.4 \mathrm{~Hz}, 1 \mathrm{H}), 7.25-7.05(\mathrm{~m}, 6 \mathrm{H}), 6.77-6.60$ $(\mathrm{m}, 3 \mathrm{H}), 6.00(\mathrm{~d}, J=8.0 \mathrm{~Hz}, 2 \mathrm{H}), 4.23-4.15\left(\mathrm{dd}, J_{1}=10.8 \mathrm{~Hz}, J_{2}=10.4 \mathrm{~Hz}, 1 \mathrm{H}\right), 4.04(\mathrm{br}, 1 \mathrm{H})$, 3.49-3.40 (m, $1 \mathrm{H}), 3.03-2.97\left(\mathrm{dd}, J_{l}=16.0 \mathrm{~Hz}, J_{2}=8.8 \mathrm{~Hz}, 1 \mathrm{H}\right)$.

${ }^{13} \mathbf{C}\left\{{ }^{1} \mathbf{H}\right\}$ NMR $\left(\mathbf{C D C l}_{3}, \mathbf{1 0 0} \mathbf{M H z}\right): \delta 159.1,147.4,146.5,146.4,146.4,146.2,146.1,145.0,141.9$, 138.4, 137.4, 136.4, 135.6, 135.3, 135.2, 133.0, 132.4, 132.4, 132.2, 132.1, 131.9, 131.8, 125.3, $124.1,123.8,123.5,123.1,123.0,123.0,122.8,122.7,121.3,119.7,117.0,112.5,55.9,30.5$.

IR $\left(\mathrm{KBr}, \mathrm{cm}^{-1}\right): 3440,3056,2690,1635,1522,1348$.

HRMS (ESI) m/z: $[\mathrm{M}+\mathrm{H}]^{+}$Calcd for $\mathrm{C}_{41} \mathrm{H}_{27} \mathrm{~N}_{6} \mathrm{O}_{8}$ 731.1885; found 731.1855.

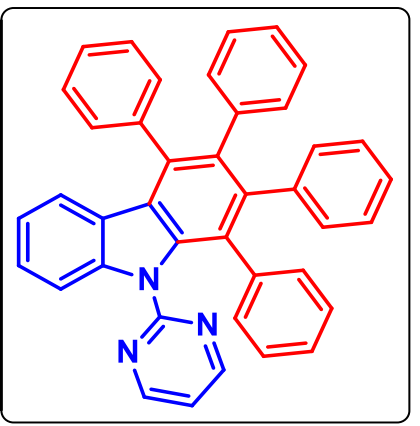

1,2,3,4-tetraphenyl-9-(pyrimidin-2-yl)-9H-carbazole (5aa): was prepared according to general procedure $(\mathbf{2 . 5})$. The crude reaction mixture was purified by column chromatography using silica gel (100-200 mesh size in 35\% EtOAc/hexane) giving 5aa (45 mg for $0.10 \mathrm{mmol}$ scale) $82 \%$ yield.

Physical State: brown solid

m.p.: $255-257^{\circ} \mathrm{C}$

$\mathbf{R}_{\boldsymbol{f}}$-value: 0.4 (20\% EtOAc/hexane)

${ }^{1} \mathbf{H}_{\text {NMR }}\left(\mathbf{C D C l}_{3}, \mathbf{4 0 0} \mathbf{M H z}\right): \delta 8.30(\mathrm{~d}, J=4.8 \mathrm{~Hz}, 2 \mathrm{H}), 7.94(\mathrm{~d}, J=8.4 \mathrm{~Hz}, 1 \mathrm{H}), 7.33-7.26(\mathrm{~m}$, $6 \mathrm{H}), 6.99-6.94(\mathrm{~m}, 3 \mathrm{H}), 6.86-6.79(\mathrm{~m}, 13 \mathrm{H}), 6.76(\mathrm{t}, J=5.2 \mathrm{~Hz}, 1 \mathrm{H}), 6.71(\mathrm{~d}, J=7.6 \mathrm{~Hz}, 1 \mathrm{H})$.

${ }^{13} \mathbf{C}\left\{{ }^{1} \mathbf{H}\right\}$ NMR $\left(\mathbf{C D C l}_{\mathbf{3}}, \mathbf{1 0 0} \mathbf{M H z}\right): \delta 152.1,148.3,142.5,140.3,140.1,139.9,139.6,137.9,137.0$, 136.8, 136.2, 136.0, 134.5, 132.0, 131.7, 131.3, 130.5, 128.7 (2C), 128.7, 127.6, 127.5, 127.4, $126.9,126.7,126.1$ (2C), 125.7 (2C), 125.2, 123.0, 122.6, 121.7, 119.8.

IR $\left(\mathrm{KBr}, \mathrm{cm}^{-1}\right): 3439,2919,1632,1415$.

HRMS (ESI) m/z: [M+Na] $]^{+}$Calcd for $\mathrm{C}_{40} \mathrm{H}_{27} \mathrm{~N}_{3} \mathrm{Na}$ 572.2097; Found 572.2096. 


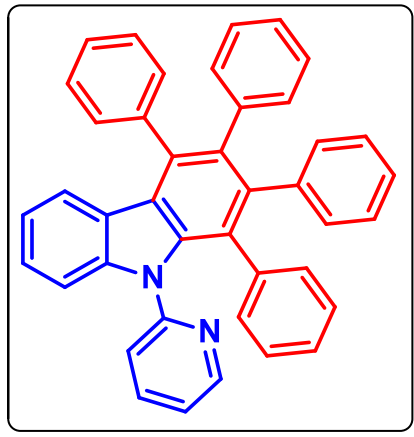

1,2,3,4-tetraphenyl-9-(pyridin-2-yl)-9H-carbazole (5ba): was prepared according to general procedure $(\mathbf{2 . 5})$. The crude reaction mixture was purified by column chromatography using silica gel (100-200 mesh size in 30\% EtOAc/hexane) giving $\mathbf{5 b a}(53 \mathrm{mg}$ for $0.10 \mathrm{mmol}$ scale) $96 \%$ yield.

Physical State: brown solid

m.p.: $268-270{ }^{\circ} \mathrm{C}$

$\mathbf{R}_{f}$-value: 0.5 (20\% EtOAc/hexane)

${ }^{1} \mathbf{H}$ NMR $\left(\mathbf{C D C l}_{3}, 400 \mathrm{MHz}\right): \delta 8.28(\mathrm{~d}, J=4.0 \mathrm{~Hz}, 1 \mathrm{H}), 7.35-7.23(\mathrm{~m}, 8 \mathrm{H}), 6.95-6.81(\mathrm{~m}, 15 \mathrm{H})$, 6.75-6.73 (m, 4H).

${ }^{13} \mathbf{C}\left\{{ }^{1} \mathbf{H}\right\}$ NMR $\left(\mathbf{C D C l}_{3}, \mathbf{1 0 0} \mathbf{M H z}\right): \delta 151.9,149.0,143.1,140.8,140.6,140.4,140.1,138.1,137.7$, $137.3,135.9,134.8,132.1,132.0,131.6,130.6,128.3,127.2,127.1,126.7$ (2C), 126.2, 125.8, $125.4,125.4,125.0,123.9,123.2,123.1,122.7,121.7,120.6,110.6$.

IR $\left(\mathrm{KBr}, \mathrm{cm}^{-1}\right):$ 3445, 3053, 1588, 1274.

HRMS (ESI) m/z: [M+Na $]^{+}$Calcd for $\mathrm{C}_{41} \mathrm{H}_{28} \mathrm{~N}_{2} \mathrm{Na}$ 571.2150; Found 571.2167.

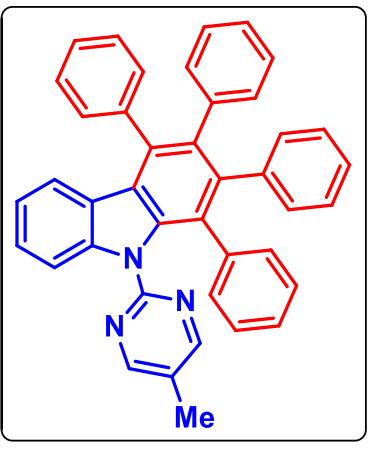

9-(5-methylpyrimidin-2-yl)-1,2,3,4-tetraphenyl-9H-carbazole (5ca): was prepared according to general procedure (2.5). The crude reaction mixture was purified by column chromatography using silica gel (100$200 \mathrm{mesh}$ size in $25 \%$ EtOAc/hexane) giving $\mathbf{5 c a}(27 \mathrm{mg}$ for $0.10 \mathrm{mmol}$ scale) $48 \%$ yield.

Physical State: yellow solid.

m.p.: $275-280{ }^{\circ} \mathrm{C}$

$\mathbf{R}_{f}$-value: 0.15 (5\% EtOAc/hexane)

${ }^{1} \mathrm{H}$ NMR $\left(\mathbf{C D C l}_{3}, 400 \mathrm{MHz}\right): \delta 8.10(\mathrm{~s}, 2 \mathrm{H}), 7.80(\mathrm{~d}, J=8.4 \mathrm{~Hz}, 1 \mathrm{H}), 7.34-7.24(\mathrm{~m}, 6 \mathrm{H}), 6.97-$ $6.92(\mathrm{~m}, 3 \mathrm{H}), 6.87-6.78(\mathrm{~m}, 13 \mathrm{H}), 6.73(\mathrm{~d}, J=8.0 \mathrm{~Hz}, 1 \mathrm{H}), 2.12(\mathrm{~s}, 3 \mathrm{H})$.

${ }^{13} \mathbf{C}\left\{{ }^{1} \mathbf{H}\right\}$ NMR $\left(\mathbf{C D C l}_{3}, \mathbf{1 0 0} \mathbf{M H z}\right): \delta 157.8,156.0,142.1,140.9,140.6,140.3,140.0,139.5,137.6$, 135.9 (2C), 135.9, 132.2, 132.1, 131.2, 130.7, 128.4, 127.3, 127.2, 126.9, 126.8, 126.4, 125.7, 125.5 (2C), 125.2, 124.8, 124.2, 122.7, 121.4, 111.3, 15.3.

IR $\left(\mathrm{KBr}, \mathrm{cm}^{-1}\right):$ 3444, 3054, 2986, 1634, 1264.

HRMS (ESI) m/z: [M+H] $]^{+}$Calcd for $\mathrm{C}_{41} \mathrm{H}_{30} \mathrm{~N}_{3}$ 564.2434; Found 564.2338. 


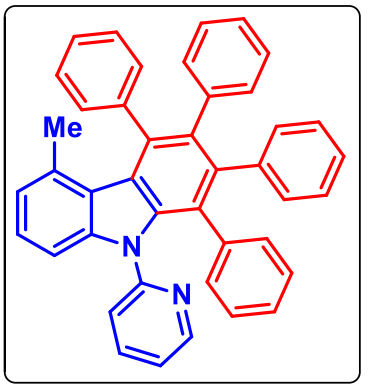

5-methyl-1,2,3,4-tetraphenyl-9-(pyridin-2-yl)-9H-carbazole (5ea): was prepared according to general procedure $(\mathbf{2 . 5})$. The crude reaction mixture was purified by column chromatography using silica gel (100200 mesh size in $20 \%$ EtOAc/hexane) giving $\mathbf{5 e a}$ (37 mg for $0.10 \mathrm{mmol}$ scale) $66 \%$ yield.

Physical State: yellow solid

Melting Point: $250-260{ }^{\circ} \mathrm{C}$

$\mathbf{R}_{f}$-value: 0.1 (5\% EtOAc/hexane)

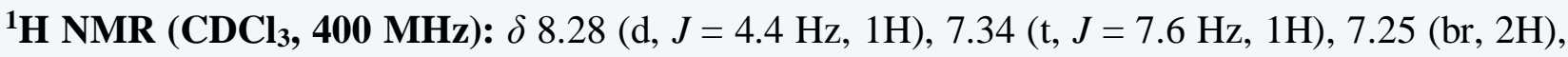
$7.20-7.14(\mathrm{~m}, 4 \mathrm{H}), 7.04(\mathrm{~d}, J=8.0 \mathrm{~Hz}, 1 \mathrm{H}), 6.96-6.91(\mathrm{~m}, 2 \mathrm{H}), 6.86-6.82(\mathrm{~m}, 6 \mathrm{H}), 6.77-6.73(\mathrm{~m}$, $10 \mathrm{H}), 1.60(\mathrm{~s}, 3 \mathrm{H})$.

${ }^{13} \mathbf{C}\left\{{ }^{1} \mathbf{H}\right\}$ NMR $\left(\mathbf{C D C l}_{\mathbf{3}}, \mathbf{1 0 0} \mathbf{M H z}\right): \delta 152.3,149.4,144.3,143.1,141.5,141.0,140.0,138.8,138.3$, $137.5,135.8,135.1,134.6,132.6,132.3,132.0,131.8,127.5,127.1,126.7,126.65,126.6,126.2$, $125.9,125.3,125.1,124.8,124.3,124.2,124.1,122.8,122.1,108.1,22.6$.

IR $\left(\mathrm{KBr}, \mathrm{cm}^{-1}\right):$ 3440, 3053, 2986, 1635, 1273.

HRMS (ESI) m/z: $[\mathrm{M}+\mathrm{H}]^{+}$Calcd for $\mathrm{C}_{42} \mathrm{H}_{31} \mathrm{~N}_{2}$ 563.2482; Found 563.2428.

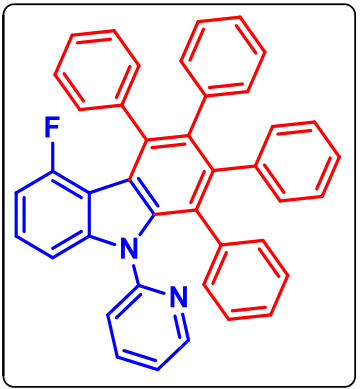

5-fluoro-1,2,3,4-tetraphenyl-9-(pyridin-2-yl)-9H-carbazole (5fa): was prepared according to general procedure (2.5). The crude reaction mixture was purified by column chromatography using silica gel (100200 mesh size in $30 \%$ EtOAc/hexane) giving $\mathbf{5 f a}(55 \mathrm{mg}$ for $0.10 \mathrm{mmol}$ scale) $97 \%$ yield.

Physical State: light yellow solid

Melting Point: $265-272{ }^{\circ} \mathrm{C}$

$\mathbf{R}_{f}$-value: 0.3 (20\% EtOAc/hexane)

${ }^{1} \mathrm{H}_{\text {NMR }}\left(\mathbf{C D C l}_{3}, \mathbf{4 0 0} \mathrm{MHz}\right): \delta 8.30(\mathrm{~d}, J=4.4 \mathrm{~Hz}, 1 \mathrm{H}), 7.34-7.17(\mathrm{~m}, 6 \mathrm{H}), 7.00(\mathrm{~d}, J=8.0 \mathrm{~Hz}$, $1 \mathrm{H}), 6.95(\mathrm{t}, J=6.4 \mathrm{~Hz}, 1 \mathrm{H}), 6.91(\mathrm{~d}, J=8.0 \mathrm{~Hz}, 1 \mathrm{H}), 6.84-6.73(\mathrm{~m}, 16 \mathrm{H}), 6.68\left(\mathrm{dd}, J_{l}=11.2 \mathrm{~Hz}\right.$, $\left.J_{2}=8.0 \mathrm{~Hz}, 1 \mathrm{H}\right)$.

${ }^{13} \mathbf{C}\left\{{ }^{1} \mathbf{H}\right\}$ NMR $\left(\mathbf{C D C l}_{3}, \mathbf{1 7 6} \mathbf{M H z}\right): \delta 157.4\left(\mathrm{~d}, J_{C-F}=253.4 \mathrm{~Hz}\right), 151.9,149.3,145.6\left(\mathrm{~d}, J_{C-F}=8.8\right.$ $\mathrm{Hz}), 142.1\left(\mathrm{~d}, J_{C-F}=5.3 \mathrm{~Hz}\right), 141.1,140.9(2 \mathrm{C}), 140.7,140.6,137.9,137.8,137.6,136.7,135.9$, 132.2, 131.9, 131.8, $131.1\left(\mathrm{~d}, J_{C-F}=3.5 \mathrm{~Hz}\right), 127.2(2 \mathrm{C}), 127.1,127.0,126.9,126.7$ (2C), 126.5, 126.0, $125.5(2 \mathrm{C}), 125.3,124.9,123.8,122.4,121.7\left(\mathrm{~d}, J_{C-F}=5.3 \mathrm{~Hz}\right), 111.7\left(\mathrm{~d}, J_{C-F}=19.4 \mathrm{~Hz}\right)$, $107.7\left(\mathrm{~d}, J_{C-F}=21.1 \mathrm{~Hz}\right), 106.5\left(\mathrm{~d}, J_{C-F}=3.5 \mathrm{~Hz}\right)$.

${ }^{19}$ F NMR ( $\left.\mathrm{CDCl}_{3}, 376 \mathrm{MHz}\right): \delta-106.0$.

IR $\left(\mathrm{KBr}, \mathrm{cm}^{-1}\right): 3440,3053,2986,1436,1273,749$.

HRMS (ESI) m/z: $[\mathrm{M}+\mathrm{H}]^{+}$Calcd for $\mathrm{C}_{41} \mathrm{H}_{28} \mathrm{FN}_{2}$ 567.2231; Found 567.2196. 


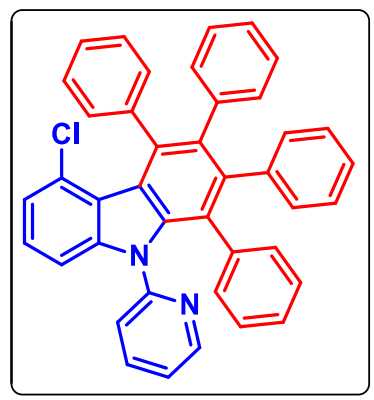

5-chloro-1,2,3,4-tetraphenyl-9-(pyridin-2-yl)-9H-carbazole (5ga): was prepared according to general procedure (2.5). The crude reaction mixture was purified by column chromatography using silica gel (100200 mesh size in 35\% EtOAc/hexane) giving 5ga (37 mg for 0.10 mmol scale) $63 \%$ yield.

Physical State: yellow solid

m.p.: $250-255^{\circ} \mathrm{C}$

$\mathbf{R}_{f}$-value: 0.3 (10\% EtOAc/hexane)

${ }^{1} \mathrm{H}$ NMR $\left(\mathbf{C D C l}_{3}, 400 \mathrm{MHz}\right): \delta 8.30\left(\mathrm{dd}, J_{l}=5.2 \mathrm{~Hz}, J_{2}=1.2 \mathrm{~Hz}, 1 \mathrm{H}\right), 7.36-7.32(\mathrm{~m}, 1 \mathrm{H}), 7.24-$ $7.22(\mathrm{~m}, 1 \mathrm{H}), 7.18-7.11(\mathrm{~m}, 3 \mathrm{H}), 7.09-7.04(\mathrm{~m}, 2 \mathrm{H}), 6.99-6.92(\mathrm{~m}, 2 \mathrm{H}), 6.85-6.82(\mathrm{~m}, 9 \mathrm{H}), 6.78-$ $6.72(\mathrm{~m}, 8 \mathrm{H})$.

${ }^{13} \mathbf{C}\left\{{ }^{1} \mathbf{H}\right\} \mathbf{N M R}\left(\mathbf{C D C l}_{3}, \mathbf{1 0 0} \mathbf{M H z}\right): \delta 151.9,149.4,145.3,142.9,141.3,141.1,140.9,140.7,140.6$, 138.9, 138.0, 137.7, 136.6, 135.8, 132.3 (2C), 131.9, 131.7 (2C), 129.1, 127.4, 127.2, 126.9, 126.7, $126.6(2 \mathrm{C}), 126.1,125.5,125.4,125.2,124.9,124.3,123.7,122.5,122.1,121.5,109.1$.

IR $\left(\mathrm{KBr}, \mathrm{cm}^{-1}\right): 3440,3053,2986,1635,1261,895$.

HRMS (ESI) m/z: $[\mathrm{M}+\mathrm{H}]^{+}$Calcd for $\mathrm{C}_{41} \mathrm{H}_{28} \mathrm{ClN}_{2}$ 583.1936; Found 583.1933.

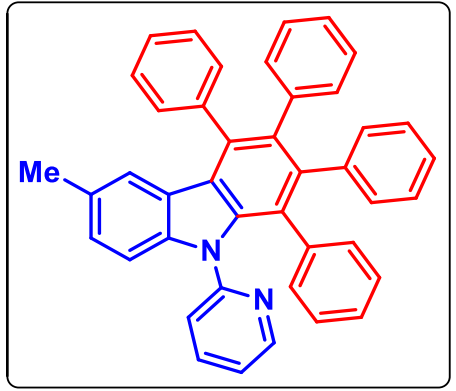

6-methyl-1,2,3,4-tetraphenyl-9-(pyridin-2-yl)-9H-carbazole (5ha): was prepared according to general procedure (2.5). The crude reaction mixture was purified by column chromatography using silica gel (100-200 mesh size in 25\% EtOAc/hexane) giving 5 ha ( $40 \mathrm{mg}$ for $0.10 \mathrm{mmol} \mathrm{scale}$ ) $72 \%$ yield.

Physical State: pale white solid

m.p.: $243-245^{\circ} \mathrm{C}$

$\mathbf{R}_{\boldsymbol{f}}$-value: 0.5 (20\% EtOAc/hexane)

${ }^{1} \mathbf{H}_{\text {NMR }}\left(\mathbf{C D C l}_{3}, \mathbf{4 0 0} \mathbf{M H z}\right): \delta 8.25(\mathrm{dd}, J=4.8 \mathrm{~Hz}, J=1.2 \mathrm{~Hz}, 1 \mathrm{H}), 7.33-7.27(\mathrm{~m}, 4 \mathrm{H}), 7.26-$ $7.21(\mathrm{~m}, 2 \mathrm{H}), 7.07(\mathrm{dd}, J=8.4 \mathrm{~Hz}, J=1.6 \mathrm{~Hz}, 1 \mathrm{H}), 6.90-6.81(\mathrm{~m}, 15 \mathrm{H}), 6.74-6.73(\mathrm{~m}, 3 \mathrm{H}), 6.49$ $(\mathrm{s}, 1 \mathrm{H}), 2.20(\mathrm{~s}, 3 \mathrm{H})$.

${ }^{13} \mathbf{C}\left\{{ }^{1} \mathbf{H}\right\}$ NMR $\left(\mathbf{C D C l}_{3}, \mathbf{1 0 0} \mathbf{M H z}\right): \delta 152.1,148.9,141.4,140.9,140.7,140.5,139.9,138.3,137.9$, 137.2, 135.9, 134.6, 132.2, 132.1, 131.7, 131.6, 130.6, 129.8, 128.2, 127.4, 127.1, 127.0, 126.9, $126.7,126.7,125.8,125.4,125.3,124.9,124.0,123.2,123.0,122.7,121.5,110.3,21.8$.

IR $\left(\mathrm{KBr}, \mathrm{cm}^{-1}\right): 3446,2922,1653,1264,698$.

HRMS (ESI) m/z: [M+Na] $]^{+}$Calcd for $\mathrm{C}_{42} \mathrm{H}_{30} \mathrm{~N}_{2} \mathrm{Na}$ 585.2307; Found 585.2288. 


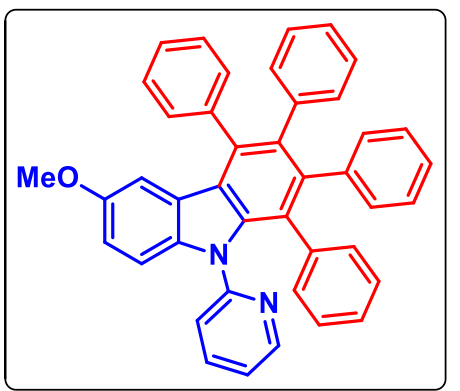

6-methoxy-1,2,3,4-tetraphenyl-9-(pyridin-2-yl)-9H-carbazole (5ia): was prepared according to general procedure (2.5). The crude reaction mixture was purified by column chromatography using silica gel (100-200 mesh size in 30\% EtOAc/hexane) giving 5ia (54 mg for $0.10 \mathrm{mmol}$ scale) $93 \%$ yield.

Physical State: brown solid

m.p.: $236-238^{\circ} \mathrm{C}$

$\mathbf{R}_{\boldsymbol{f}}$-value: 0.4 (20\% EtOAc/hexane)

${ }^{1} \mathbf{H}$ NMR $\left(\mathbf{C D C l}_{3}, 400 \mathrm{MHz}\right): \delta 8.25(\mathrm{dd}, J=4.8 \mathrm{~Hz}, J=1.2 \mathrm{~Hz}, 1 \mathrm{H}), 7.38-7.31(\mathrm{~m}, 4 \mathrm{H}), 7.28-$ $7.22(\mathrm{~m}, 3 \mathrm{H}), 6.92-6.81(\mathrm{~m}, 15 \mathrm{H}), 6.75-6.72(\mathrm{~m}, 3 \mathrm{H}), 6.17(\mathrm{~d}, J=2.4 \mathrm{~Hz}, 1 \mathrm{H}), 3.46(\mathrm{~s}, 3 \mathrm{H})$.

${ }^{13} \mathbf{C}\left\{{ }^{1} \mathbf{H}\right\}$ NMR $\left(\mathbf{C D C l}_{3}, \mathbf{1 0 0} \mathbf{M H z}\right): \delta 154.3,152.0,148.9,140.8,140.6,140.4,140.0,138.2,138.1$, $138.0,137.2$, 135.8, 134.5, 132.2, 132.1, 131.6, 130.8, 128.3, 127.2, 127.1, 126.8, 126.7, 125.9, $125.5,125.1,124.4,123.2,122.9,121.5,115.4,111.5,105.0,55.5$.

IR $\left(\mathrm{KBr}, \mathrm{cm}^{-1}\right):$ 3441, 3053, 3021, 1635, 1329, 1216.

HRMS (ESI) m/z: $[\mathrm{M}+\mathrm{H}]^{+}$Calcd for $\mathrm{C}_{42} \mathrm{H}_{31} \mathrm{~N}_{2} \mathrm{O}$ 579.2431; Found 579.2421.

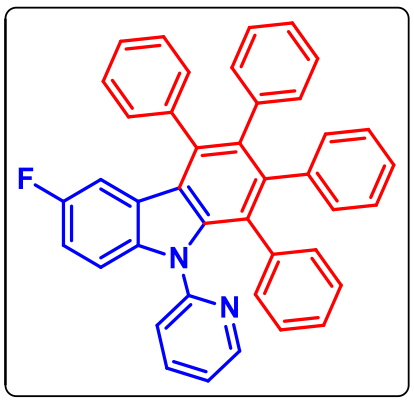

6-fluoro-1,2,3,4-tetraphenyl-9-(pyridin-2-yl)-9H-carbazole (5ja): was prepared according to general procedure (2.5). The crude reaction mixture was purified by column chromatography using silica gel (100-200 mesh size in 25\% EtOAc/hexane) giving 5ja (35 mg for 0.10 mmol scale) $62 \%$ yield.

Physical State: white solid

m.p.: $285-290{ }^{\circ} \mathrm{C}$

$\mathbf{R}_{\boldsymbol{f}}$-value: 0.4 (20\% EtOAc/hexane)

${ }^{1} \mathbf{H}$ NMR (CDCl 3 , $\left.400 \mathrm{MHz}\right): \delta 8.29(\mathrm{~d}, J=4.4 \mathrm{~Hz}, 1 \mathrm{H}), 7.32-7.25(\mathrm{~m}, 7 \mathrm{H}), 7.01-6.74(\mathrm{~m}, 18 \mathrm{H})$, 6.40-6.37 (m, 1H).

${ }^{13} \mathbf{C}\left\{{ }^{1} \mathbf{H}\right\}$ NMR $\left(\mathbf{C D C l}_{3}, \mathbf{1 0 0} \mathbf{M H z}\right): \delta 158.0(\mathrm{~d}, J=234.0 \mathrm{~Hz}), 151.8,149.0,140.8,140.6,140.4$, 139.8, 139.4, 138.5, 137.9, 137.4, 136.1, 134.9, 132.1, 132.0, 131.5, 130.4, 128.5, 127.4, 127.3, $126.83,126.8,126.0,125.5(\mathrm{~d}, J=3.0 \mathrm{~Hz}), 125.2,124.5$ (d, $J=10 \mathrm{~Hz}), 123.1,122.8(\mathrm{~d}, J=3.0$ $\mathrm{Hz}), 121.9,113.9(\mathrm{~d}, J=25.0 \mathrm{~Hz}), 111.4(\mathrm{~d}, J=9.0 \mathrm{~Hz}), 108.4(\mathrm{~d}, J=25.0 \mathrm{~Hz})$.

${ }^{19}$ F NMR $\left(\mathrm{CDCl}_{3}, 376 \mathrm{MHz}\right): \delta-122.8$.

IR $\left(\mathrm{KBr}, \mathrm{cm}^{-1}\right): 3440,3054,2986,2305,1635,1261,895$.

HRMS (ESI) m/z: $[\mathrm{M}+\mathrm{H}]^{+}$Calcd for $\mathrm{C}_{41} \mathrm{H}_{28} \mathrm{FN}_{2}$ 567.2231; Found 567.2185. 


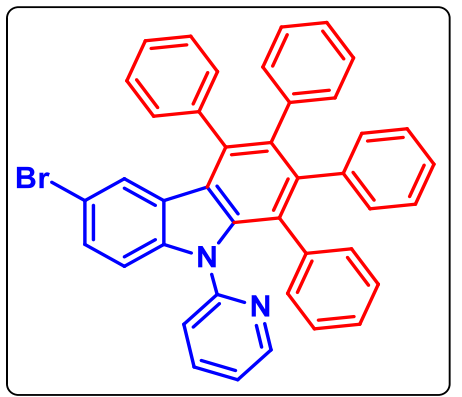

6-bromo-1,2,3,4-tetraphenyl-9-(pyridin-2-yl)-9H-carbazole (5ka): was prepared according to general procedure (2.5). The crude reaction mixture was purified by column chromatography using silica gel (100-200 mesh size in 20\% EtOAc/hexane) giving $5 \mathbf{k a ~ ( 5 0 ~ m g ~ f o r ~} 0.10 \mathrm{mmol}$ scale) $80 \%$ yield.

Physical State: light green solid

m.p.: $245-250{ }^{\circ} \mathrm{C}$

$\mathbf{R}_{f}$-value: 0.2 (5\% EtOAc/hexane)

${ }^{1} \mathbf{H}$ NMR $\left(\mathbf{C D C l}_{3}, \mathbf{4 0 0} \mathbf{M H z}\right): \delta 8.30\left(\mathrm{dd}, J_{1}=4.8 \mathrm{~Hz}, J_{2}=1.2 \mathrm{~Hz}, 1 \mathrm{H}\right), 7.33-7.28(\mathrm{~m}, 3 \mathrm{H}), 7.22-$ $7.17(\mathrm{~m}, 4 \mathrm{H}), 7.00(\mathrm{~d}, J=8.4 \mathrm{~Hz}, 1 \mathrm{H}), 6.96-6.93(\mathrm{~m}, 1 \mathrm{H}), 6.90(\mathrm{~d}, J=8.0 \mathrm{~Hz}, 1 \mathrm{H}), 6.86-6.73(\mathrm{~m}$, $15 \mathrm{H}), 6.71-6.66(\mathrm{~m}, 1 \mathrm{H})$.

${ }^{13} \mathbf{C}\left\{{ }^{1} \mathbf{H}\right\}$ NMR (CDCl $\left.3,100 \mathrm{MHz}\right): \delta 158.8,156.3,152.0,149.3,145.8,145.7,142.14,142.1$, 141.2 , 140.9, 140.8, 138.0, 137.9, 137.6, 136.8, 135.9, 132.2, 131.9, 131.8, 131.14, 131.1, 127.2, $127.15,127.1,126.7,126.68,126.5,126.0,125.5,125.3,125.0,123.9,122.4,121.8,121.75,111.9$, $111.8,107.8,107.6,106.6,106.5$.

IR $\left(\mathrm{KBr}, \mathrm{cm}^{-1}\right): 3441,2084,1636,1383,1329,695$.

HRMS (ESI) m/z: $[\mathrm{M}+\mathrm{H}]^{+}$Calcd for $\mathrm{C}_{41} \mathrm{H}_{28} \mathrm{BrN}_{2}$ 627.1436; Found 627.1414.

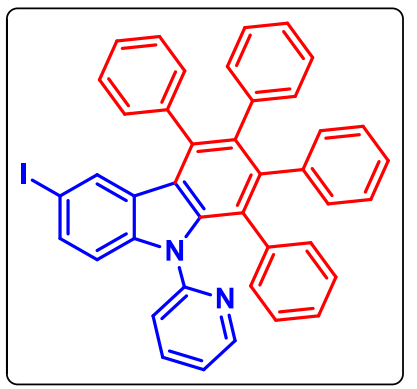

6-iodo-1,2,3,4-tetraphenyl-9-(pyridin-2-yl)-9H-carbazole (5la): was prepared according to general procedure (2.5). The crude reaction mixture was purified by column chromatography using silica gel (100-200 mesh size in 35\% EtOAc/hexane) giving 5la (45 $\mathrm{mg}$ for $0.10 \mathrm{mmol}$ scale) $67 \%$ yield.

Physical State: brown solid

m.p.: $276-278{ }^{\circ} \mathrm{C}$

$\mathbf{R}_{\boldsymbol{f}}$-value: 0.3 (20\% EtOAc/hexane)

${ }^{1} \mathbf{H}_{\text {NMR }}\left(\mathbf{C D C l}_{3}, \mathbf{4 0 0} \mathbf{M H z}\right): \delta 8.28(\mathrm{dd}, J=5.2 \mathrm{~Hz}, J=1.6 \mathrm{~Hz}, 1 \mathrm{H}), 7.49(\mathrm{dd}, J=8.0 \mathrm{~Hz}, J=2.0$ $\mathrm{Hz}, 1 \mathrm{H}), 7.35-7.27(\mathrm{~m}, 6 \mathrm{H}), 7.07(\mathrm{~d}, J=8.4 \mathrm{~Hz}, 1 \mathrm{H}), 6.97-7.92(\mathrm{~m}, 2 \mathrm{H}), 6.88-6.80(\mathrm{~m}, 13 \mathrm{H}), 6.76-$ $6.73(\mathrm{~m}, 3 \mathrm{H})$.

${ }^{13} \mathbf{C}\left\{{ }^{1} \mathbf{H}\right\}$ NMR $\left(\mathbf{C D C l}_{3}, \mathbf{1 0 0} \mathbf{M H z}\right): \delta 151.4,149.0,142.2,140.9,140.5,140.3,139.8,137.8,137.6$, $137.4,136.1,135.1,134.4,132.0,131.9,131.6,131.5,130.3,130.1,128.5,127.4,127.2,126.8$, $126.7,126.3,126.0,125.5,125.1,123.1,122.1,122.0,112.7,83.8$.

IR $\left(\mathrm{KBr}, \mathrm{cm}^{-1}\right): 3440,3054,2923,1599,1380,698$.

HRMS (ESI) m/z: [M+Na] ${ }^{+}$Calcd for $\mathrm{C}_{41} \mathrm{H}_{27} \mathrm{IN}_{2} \mathrm{Na}$ 697.1111; Found 697.1116. 


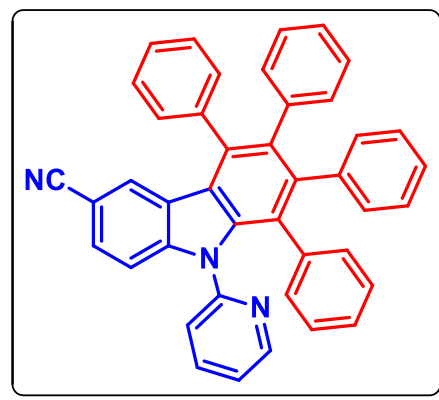

5,6,7,8-tetraphenyl-9-(pyridin-2-yl)-9H-carbazole-3-carbonitrile

(5ma): was prepared according to general procedure (2.5). The crude reaction mixture was purified by column chromatography using silica gel (100-200 mesh size in 40\% EtOAc/hexane) giving 5ma (26 mg for $0.10 \mathrm{mmol}$ scale) $46 \%$ yield.

Physical State: brown solid

m.p.: $272-274{ }^{\circ} \mathrm{C}$

$\mathbf{R}_{f}$-value: 0.4 (20\% EtOAc/hexane)

${ }^{1} \mathbf{H}$ NMR $\left(\mathbf{C D C l}_{3}, \mathbf{4 0 0} \mathbf{M H z}\right): \delta 8.33-8.32(\mathrm{~m}, 1 \mathrm{H}), 7.50-7.47(\mathrm{~m}, 1 \mathrm{H}), 7.37-7.25(\mathrm{~m}, 7 \mathrm{H}), 7.02-$ $6.98(\mathrm{~m}, 2 \mathrm{H}), 6.87-6.85(\mathrm{~m}, 5 \mathrm{H}), 6.84-6.81(\mathrm{~m}, 7 \mathrm{H}), 6.79-6.74(\mathrm{~m}, 4 \mathrm{H})$.

${ }^{13} \mathbf{C}\left\{{ }^{1} \mathbf{H}\right\}$ NMR $\left(\mathbf{C D C l}_{3}, \mathbf{1 0 0} \mathbf{M H z}\right): \delta 150.9,149.2,144.7,141.8,140.2,140.0,139.4,137.6,137.4$, $136.4,136.0,131.9,131.8,131.7,131.5,130.1,129.4$, 128.8, 127.9, 127.6, 127.3, 126.9, 126.9, $126.8,126.2,125.7,125.5,124.1,123.4,122.6,121.9,120.7,111.5,103.5$.

IR $\left(\mathrm{KBr}, \mathrm{cm}^{-1}\right): 3441,3055,1601,1383,1299$.

HRMS (ESI) m/z: $[\mathrm{M}+\mathrm{H}]^{+}$Calcd for $\mathrm{C}_{42} \mathrm{H}_{28} \mathrm{~N}_{3}$ 574.2278; Found 574.2334.

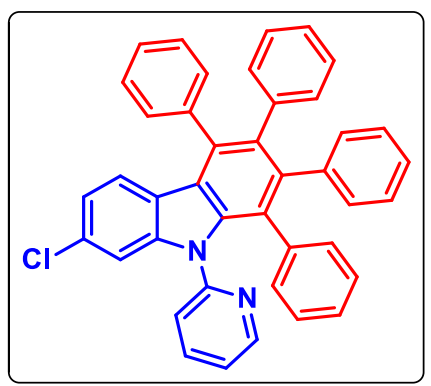

7-chloro-1,2,3,4-tetraphenyl-9-(pyridin-2-yl)-9H-carbazole (5na): was prepared according to general procedure (2.5). The crude reaction mixture was purified by column chromatography using silica gel (100200 mesh size in $15 \%$ EtOAc/hexane) giving 5 na (38 $\mathrm{mg}$ for 0.10 mmol scale) $65 \%$ yield.

Physical State: yellow solid m.p.: $278-281^{\circ} \mathrm{C}$

$\mathbf{R}_{f}$-value: 0.5 (10\% EtOAc/hexane)

${ }^{1} \mathbf{H}$ NMR $\left(\mathbf{C D C l}_{3}, \mathbf{4 0 0} \mathbf{M H z}\right): \delta 8.30(\mathrm{~d}, J=4.4 \mathrm{~Hz}, 1 \mathrm{H}), 7.32-7.25(\mathrm{~m}, 6 \mathrm{H}), 6.96-6.93(\mathrm{~m}, 1 \mathrm{H})$, 6.91-6.74 (m, 18H), $6.64(\mathrm{~d}, J=8.8 \mathrm{~Hz}, 1 \mathrm{H})$.

${ }^{13} \mathbf{C}\left\{{ }^{1} \mathbf{H}\right\}$ NMR (CDCl $\left.3,100 \mathbf{M H z}\right): \delta 151.4,149.2,143.6,141.0,140.6,140.4,140.1,138.0,137.9$, $137.5,135.9,135.3,132.1,132.0$ (2C), 131.8, 131.6, 130.5, 128.5, 127.3 (2C), 126.9, 126.8 (2C), $126.1,125.6,125.5,125.2,123.5,123.2,122.61,122.6,122.1,121.2,110.9$.

IR $\left(\mathrm{KBr}, \mathrm{cm}^{-1}\right): 3421,3053,1601,1273,702$.

HRMS (ESI) m/z: [M+Na $]^{+}$Calcd for $\mathrm{C}_{41} \mathrm{H}_{27} \mathrm{ClN}_{2} \mathrm{Na}$ 605.1755; Found 605.1758. 


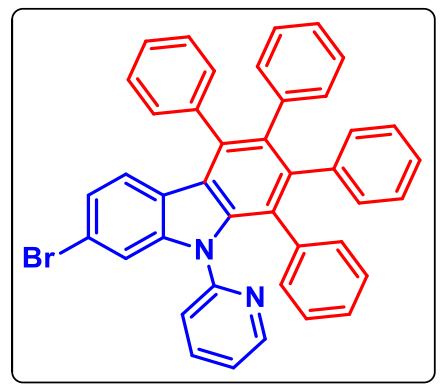

7-bromo-1,2,3,4-tetraphenyl-9-(pyridin-2-yl)-9H-carbazole (5oa): was prepared according to general procedure (2.5). The crude reaction mixture was purified by column chromatography using silica gel (100200 mesh size in 30\% EtOAc/hexane) giving 5oa (43 mg for 0.10 mmol scale) $69 \%$ yield.

Physical State: yellow solid.

Melting Point: $270-274^{\circ} \mathrm{C}$

$\mathbf{R}_{f}$-value: 0.3 (20\% EtOAc/hexane)

${ }^{1} \mathbf{H}$ NMR $\left(\mathbf{C D C l}_{\mathbf{3}}, \mathbf{4 0 0} \mathbf{M H z}\right): \delta 8.31(\mathrm{~d}, J=4.4 \mathrm{~Hz}, 1 \mathrm{H}), 7.62(\mathrm{~s}, 1 \mathrm{H}), 7.31-7.22(\mathrm{~m}, 9 \mathrm{H}), 6.97-$ $6.94(\mathrm{~m}, 1 \mathrm{H}), 6.87-6.74(\mathrm{~m}, 14 \mathrm{H}), 6.45(\mathrm{~d}, J=8.4 \mathrm{~Hz}, 1 \mathrm{H})$.

${ }^{13} \mathbf{C}\left\{{ }^{1} \mathbf{H}\right\}$ NMR $\left(\mathbf{C D C l}_{3}, \mathbf{1 0 0} \mathbf{M H z}\right): \delta 151.4,149.2,143.9,143.8,140.9,140.7,140.6,140.4,140.1$, $137.8,137.5,136.0,135.9,135.3,132.1,132.0,131.5,130.5$ (2C), 129.7, 128.5, 127.31, 127.3, 126.8 (2C), 126.0, 125.6, 125.5, 125.2 (2C), 125.2, 124.1, 124.0, 123.8, 123.5, 123.2 (2C), 122.9, $122.6(2 \mathrm{C}), 122.2,120.0,119.6,113.8,91.1$.

IR $\left(\mathrm{KBr}, \mathrm{cm}^{-1}\right): 3053,2986,1601,1264,1154,738$.

HRMS (ESI) m/z: [M+H] $]^{+}$Calcd for $\mathrm{C}_{41} \mathrm{H}_{28} \mathrm{BrN}_{2}$ 627.1436; Found 627.1413.

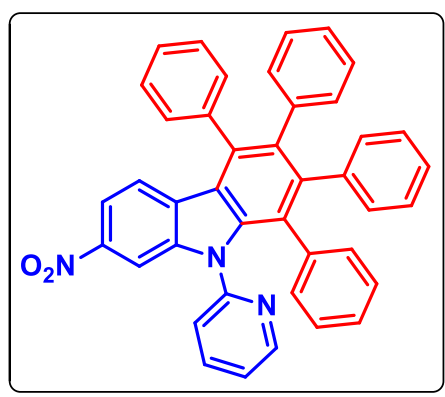

7-nitro-1,2,3,4-tetraphenyl-9-(pyridin-2-yl)-9H-carbazole (5pa): was prepared according to general procedure (2.5). The crude reaction mixture was purified by column chromatography using silica gel (100-200 mesh size in 25\% EtOAc/hexane) giving 5pa (49 mg for $0.10 \mathrm{mmol}$ scale) $83 \%$ yield.

Physical State: yellow solid

m.p.: $296-298^{\circ} \mathrm{C}$

$\mathbf{R}_{f}$-value: 0.2 (5\% EtOAc/hexane)

${ }^{1} \mathbf{H}_{\mathrm{NMR}}\left(\mathbf{C D C l}_{3}, \mathbf{4 0 0} \mathrm{MHz}\right): \delta 8.34\left(\mathrm{dd}, J_{l}=4.8 \mathrm{~Hz}, J_{2}=1.2 \mathrm{~Hz}, 1 \mathrm{H}\right), 8.14(\mathrm{~d}, J=2.0 \mathrm{~Hz}, 1 \mathrm{H})$, $7.83\left(\mathrm{dd}, J_{l}=8.8 \mathrm{~Hz}, J_{2}=2.0 \mathrm{~Hz}, 1 \mathrm{H}\right), 7.37-7.30(\mathrm{~m}, 6 \mathrm{H}), 7.03-7.00(\mathrm{~m}, 1 \mathrm{H}), 6.90-6.75(\mathrm{~m}, 17 \mathrm{H})$.

${ }^{13} \mathbf{C}\left\{{ }^{1} \mathbf{H}\right\}$ NMR $\left(\mathbf{C D C l}_{3}, \mathbf{1 0 0} \mathbf{M H z}\right): \delta 150.8,149.5,146.0,142.7,142.1,140.2,139.92,139.9$, 139.6, 137.8, 137.3, 137.0, 136.0, 132.0, 131.7, 131.5, 130.3, 129.1, 128.7, 127.7, 127.4, 127.0, $126.9,126.3,125.82,125.8,125.6,123.3,122.7,121.8,115.9,106.9$.

IR $\left(\mathrm{KBr}, \mathrm{cm}^{-1}\right): 3943,3688,3420,1518,1421$.

HRMS (ESI) m/z: [M+H] $]^{+}$Calcd for $\mathrm{C}_{41} \mathrm{H}_{28} \mathrm{~N}_{3} \mathrm{O}_{2}$ 594.2176; Found 594.2149. 


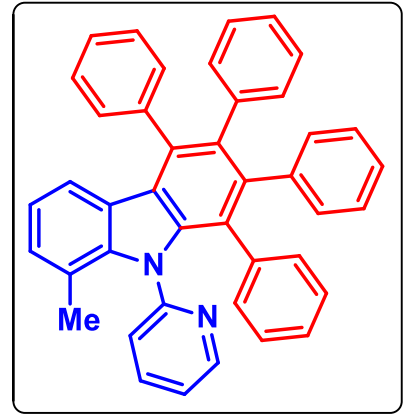

8-methyl-1,2,3,4-tetraphenyl-9-(pyridin-2-yl)-9H-carbazole (5qa): was prepared according to general procedure (2.5). The crude reaction mixture was purified by column chromatography using silica gel (100200 mesh size in 25\% EtOAc/hexane) giving 5qa (31 mg for 0.10 mmol scale) $55 \%$ yield.

Physical State: yellow liquid

$\mathbf{R}_{f}$-value: 0.5 (20\% EtOAc/hexane)

${ }^{1} \mathbf{H}_{\text {NMR }}\left(\mathbf{C D C l}_{3}, 400 \mathrm{MHz}\right): \delta 8.25\left(\mathrm{dd}, \boldsymbol{J}_{1}=4.8 \mathrm{~Hz}, J_{2}=2.0 \mathrm{~Hz}, 1 \mathrm{H}\right), 7.35-7.25(\mathrm{~m}, 5 \mathrm{H}), 7.23-$ $7.19(\mathrm{~m}, 1 \mathrm{H}), 7.01-6.95(\mathrm{~m}, 3 \mathrm{H}), 6.89-6.73(\mathrm{~m}, 16 \mathrm{H}), 6.63(\mathrm{~d}, J=8.0 \mathrm{~Hz}, 1 \mathrm{H}), 1.66(\mathrm{~s}, 3 \mathrm{H})$.

${ }^{13} \mathbf{C}\left\{{ }^{1} \mathbf{H}\right\}$ NMR $\left(\mathbf{C D C l}_{3}, \mathbf{1 0 0} \mathbf{M H z}\right): \delta 154.7,148.5,142.7,140.8,140.6,140.5,140.4,140.0,138.4$, 136.8, 135.6, 134.5, 132.1, 131.7, 131.6, 130.6, 129.1, 128.3, 127.1, 127.0, 126.6, 126.6, 126.5, $126.2,125.7,125.6,125.3,124.9,123.0,122.7,121.8,120.8,120.5,19.6$.

IR $\left(\mathrm{KBr}, \mathrm{cm}^{-1}\right): 3440,3055,1642,1406$.

HRMS (ESI) m/z: [M+H] $]^{+}$Calcd for $\mathrm{C}_{42} \mathrm{H}_{31} \mathrm{~N}_{2}$ 563.2482; Found 563.2497.

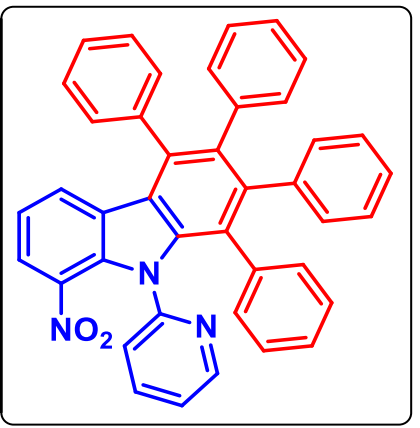

8-nitro-1,2,3,4-tetraphenyl-9-(pyridin-2-yl)-9H-carbazole (5ra): was prepared according to general procedure (2.5). The crude reaction mixture was purified by column chromatography using silica gel (100-200 mesh size in 25\% EtOAc/hexane) giving 5ra (53 $\mathrm{mg}$ for $0.10 \mathrm{mmol}$ scale) $89 \%$ yield.

Physical State: brown liquid

$\mathbf{R}_{f}$-value: 0.4 (20\% EtOAc/hexane)

${ }^{1} \mathbf{H}$ NMR $\left(\mathbf{C D C l}_{3}, \mathbf{4 0 0} \mathbf{M H z}\right): \delta 8.18\left(\mathrm{dd}, J_{l}=5.2 \mathrm{~Hz}, J_{2}=1.2 \mathrm{~Hz}, 1 \mathrm{H}\right), 7.72(\mathrm{p}, J=8.4 \mathrm{~Hz}, J=4.0$ $\mathrm{Hz}, 1 \mathrm{H}), 7.33-7.29(\mathrm{~m}, 5 \mathrm{H}), 7.12-7.07(\mathrm{~m}, 1 \mathrm{H}), 6.95-6.92(\mathrm{~m}, 3 \mathrm{H}), 6.86-6.79(\mathrm{~m}, 7 \mathrm{H}), 6.76-6.68(\mathrm{~m}$, 9H).

${ }^{13} \mathbf{C}\left\{{ }^{1} \mathbf{H}\right\}$ NMR $\left(\mathbf{C D C l}_{3}, \mathbf{1 0 0} \mathbf{M H z}\right): \delta 152.1,148.3,142.5,140.3,140.1,139.9,139.6,137.9,137.0$, 136.8, 136.2, 136.0, 134.5, 132.0, 131.7, 131.3, 130.5, 128.7(2C), 127.6, 127.5, 127.4, 126.9, 126.7, $126.1,126.1,125.7,125.6,125.2,123.0,122.6,121.7,119.8$.

IR $\left(\mathrm{KBr}, \mathrm{cm}^{-1}\right):$ 3440, 3055, 2923, 1590, 1378.

HRMS (ESI) m/z: $[\mathrm{M}+\mathrm{H}]^{+}$Calcd for $\mathrm{C}_{41} \mathrm{H}_{28} \mathrm{~N}_{3} \mathrm{O}_{2}$ 594.2176; Found 594.2179. 


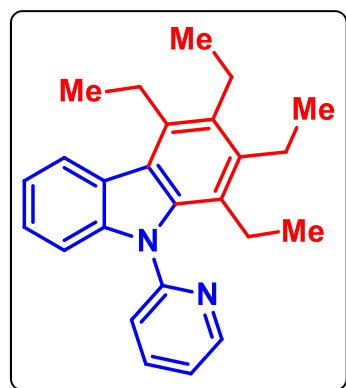

1,2,3,4-tetraethyl-9-(pyridin-2-yl)-9H-carbazole (5bb): was prepared according to general procedure (2.5). The crude reaction mixture was purified by column chromatography using silica gel (100-200 mesh size in $15 \%$ EtOAc/hexane) giving $\mathbf{5 b b}$ (30 $\mathrm{mg}$ for $0.10 \mathrm{mmol} \mathrm{scale}$ ) $84 \%$ yield.

Physical State: colorless liquid

$\mathbf{R}_{f}$-value: 0.5 (10\% EtOAc/hexane)

${ }^{1} \mathbf{H}$ NMR $\left(\mathbf{C D C l}_{3}, 400 \mathrm{MHz}\right): \delta 8.69\left(\mathrm{dd}, J_{1}=4.8 \mathrm{~Hz}, J_{2}=1.6 \mathrm{~Hz}, 1 \mathrm{H}\right), 8.14(\mathrm{~d}, J=6.8 \mathrm{~Hz}, 1 \mathrm{H})$, 7.91-7.87 (m, 1H), $7.44(\mathrm{~d}, J=8.0 \mathrm{~Hz}, 1 \mathrm{H}), 7.41-7.38(\mathrm{~m}, 1 \mathrm{H}), 7.28-7.20(\mathrm{~m}, 2 \mathrm{H}), 7.03(\mathrm{~d}, J=7.2$ $\mathrm{Hz}, 1 \mathrm{H}), 3.30(\mathrm{q}, J=7.6 \mathrm{~Hz}, 2 \mathrm{H}), 288-2.76(\mathrm{~m}, 4 \mathrm{H}), 2.39(\mathrm{q}, J=7.2 \mathrm{~Hz}, 2 \mathrm{H}), 1.44(\mathrm{t}, J=7.2 \mathrm{~Hz}$, $3 \mathrm{H}), 1.28(\mathrm{t}, J=7.6 \mathrm{~Hz}, 3 \mathrm{H}), 1.20(\mathrm{t}, J=7.2 \mathrm{~Hz}, 3 \mathrm{H}), 0.86(\mathrm{t}, J=7.2 \mathrm{~Hz}, 3 \mathrm{H})$.

${ }^{13} \mathbf{C}\left\{{ }^{1} \mathbf{H}\right\}$ NMR $\left(\mathbf{C D C l}_{3}, \mathbf{1 0 0} \mathbf{M H z}\right): \delta 154.6,150.1,143.8,139.6,139.2,138.5,135.5,133.2,125.0$, 124.4, 124.2, 124.0, 123.4, 122.7, 122.0, 120.6, 110.4, 77.4, 23.4, 22.7, 22.1, 21.4, 16.8, 16.5, 15.2, 14.7.

IR $\left(\mathrm{KBr}, \mathrm{cm}^{-1}\right): 3442,3049,2869,1585,1465,1398$.

HRMS (ESI) m/z: [M+H] $]^{+}$Calcd for $\mathrm{C}_{25} \mathrm{H}_{29} \mathrm{~N}_{2}$ 357.2325; Found 357.2321.

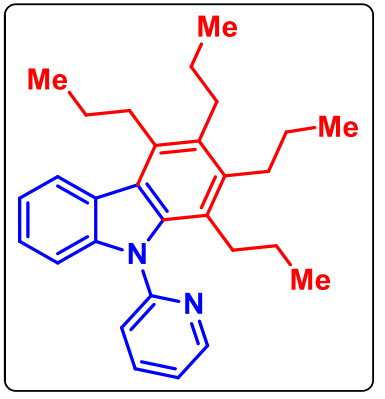

1,2,3,4-tetrapropyl-9-(pyridin-2-yl)-9H-carbazole (5bc): was prepared according to general procedure (2.5). The crude reaction mixture was purified by column chromatography using silica gel (100200 mesh size in $10 \%$ EtOAc/hexane) giving $\mathbf{5 b c}$ (31 $\mathrm{mg}$ for $0.10 \mathrm{mmol}$ scale) $76 \%$ yield.

Physical State: brown liquid $\mathbf{R}_{f}$-value: 0.6 (10\% EtOAc/hexane)

${ }^{1} \mathbf{H}$ NMR $\left(\mathbf{C D C l}_{3}, \mathbf{4 0 0} \mathbf{M H z}\right): \delta 8.70-8.68(\mathrm{~m}, 1 \mathrm{H}), 8.04(\mathrm{~d}, J=7.2 \mathrm{~Hz}, 1 \mathrm{H}), 7.88\left(\mathrm{td}, J_{1}=7.6 \mathrm{~Hz}\right.$, $\left.J_{2}=2.0 \mathrm{~Hz} 1 \mathrm{H}\right), 7.43-7.38(\mathrm{~m}, 2 \mathrm{H}), 7.27-7.19(\mathrm{~m}, 2 \mathrm{H}), 7.02-7.00(\mathrm{~m}, 1 \mathrm{H}), 3.20-3.16(\mathrm{~m}, 2 \mathrm{H}), 2.75-$ $2.64(\mathrm{~m}, 4 \mathrm{H}), 2.26(\mathrm{t}, J=8.4 \mathrm{~Hz}, 2 \mathrm{H}), 1.85-1.79(\mathrm{~m}, 2 \mathrm{H}), 1.65-1.50(\mathrm{~m}, 4 \mathrm{H}), 1.36-1.30(\mathrm{~m}, 2 \mathrm{H})$, $1.21(\mathrm{t}, J=7.62 \mathrm{~Hz}, 3 \mathrm{H}), 1.10(\mathrm{t}, J=7.2 \mathrm{~Hz}, 3 \mathrm{H}), 1.04(\mathrm{t}, J=7.2 \mathrm{~Hz}, 3 \mathrm{H}), 0.50(\mathrm{t}, J=7.2 \mathrm{~Hz}, 3 \mathrm{H})$.

${ }^{13} \mathbf{C}\left\{{ }^{1} \mathbf{H}\right\}$ NMR (CDC13, $\left.100 \mathrm{MHz}\right): \delta 154.5,150.1,143.7,139.2,138.6,138.5,134.2,132.3,124.9$, $124.5,124.1,123.2,122.9,122.5,122.1,120.5,110.3,32.9,32.5,31.9,30.9,25.8,25.6,24.0,23.5$, $15.4,15.3,15.1,14.6$.

IR $\left(\mathrm{KBr}, \mathrm{cm}^{-1}\right): 3441,2956,2927,1635,1465,1397,1345$.

HRMS (ESI) m/z: [M+H] $]^{+}$Calcd for $\mathrm{C}_{29} \mathrm{H}_{37} \mathrm{~N}_{2}$ 413.2951; Found 413.2935. 


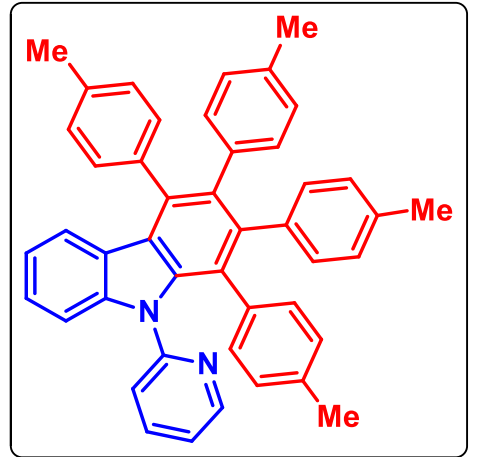
silica gel (100-200 mesh size in 25\% EtOAc/hexane) giving 5bd (44 $\mathrm{mg}$ for $0.10 \mathrm{mmol} \mathrm{scale)} 73 \%$ yield.

Physical State: brown solid

m.p.: $261-262{ }^{\circ} \mathrm{C}$

$\mathbf{R}_{f}$-value: 0.5 (20\% EtOAc/hexane)

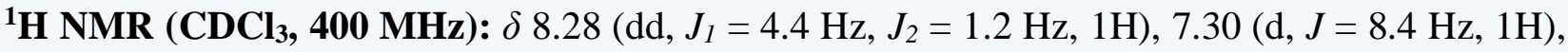
7.25-7.19 (m, 4H), $7.11(\mathrm{~d}, J=7.6 \mathrm{~Hz}, 2 \mathrm{H}), 6.94-6.90(\mathrm{~m}, 2 \mathrm{H}), 6.77-6.62(\mathrm{~m}, 12 \mathrm{H}), 6.51(\mathrm{~d}, J=$ $8.0 \mathrm{~Hz}, 2 \mathrm{H}), 2.7$ (s, 3H), 2.10-2.07 (m, 9H).

${ }^{13} \mathbf{C}\left\{{ }^{1} \mathbf{H}\right\}$ NMR $\left(\mathbf{C D C l}_{\mathbf{3}}, \mathbf{1 0 0} \mathbf{M H z}\right): \delta 152.1,148.7,143.0,140.2,137.9,137.9,137.8,137.6,137.0$, 136.3, 135.9, 135.3, 135.0, 135.0, 134.4, 134.3, 131.9, 131.8, 131.4, 130.4, 129.0, 127.9, 127.4, $127.4,125.9,124.9,124.1,123.3,123.2,122.7,121.1,120.5,110.5,21.7,21.4,21.4,21.3$.

IR $\left(\mathrm{KBr}, \mathrm{cm}^{-1}\right): 3441,1635,1515,1435,1337,1299$.

HRMS (ESI) m/z: [M+H] $]^{+}$Calcd for $\mathrm{C}_{45} \mathrm{H}_{37} \mathrm{~N}_{2}$ 605.2951; Found 605.2896.

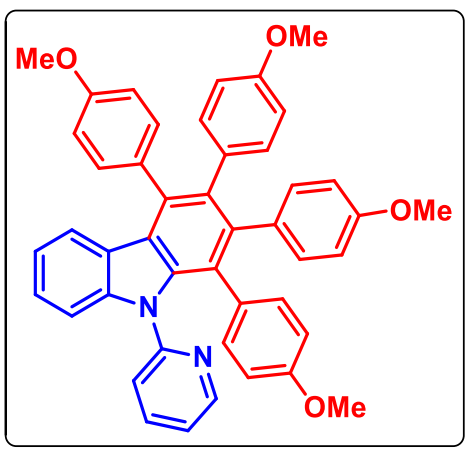

1,2,3,4-tetrakis(4-methoxyphenyl)-9-(pyridin-2-yl)-9Hcarbazole (5be): was prepared according to general procedure (2.5). The crude reaction mixture was purified by column chromatography using silica gel (100-200 mesh size in $40 \%$ EtOAc/hexane) giving 5 be (58 $\mathrm{mg}$ for $0.10 \mathrm{mmol} \mathrm{scale}$ ) $86 \%$ yield.

Physical State: yellow solid

m.p.: $271-273^{\circ} \mathrm{C}$

$\mathbf{R}_{f}$-value: 0.3 (20\% EtOAc/hexane)

${ }^{1} \mathbf{H}_{\text {NMR }}\left(\mathbf{C D C l}_{3}, \mathbf{4 0 0} \mathbf{M H z}\right): \delta 8.31(\mathrm{~d}, J=4.4 \mathrm{~Hz}, 1 \mathrm{H}), 7.33-7.21(\mathrm{~m}, 5 \mathrm{H}), 6.96-6.92(\mathrm{~m}, 2 \mathrm{H})$, 6.87-6.67 (m, 10H), 6.45-6.39 (m, 4H), $6.29(\mathrm{~d}, J=8.4 \mathrm{~Hz}, 2 \mathrm{H}), 3.84(\mathrm{~s}, 3 \mathrm{H}) 3.64-3.61(\mathrm{~m}, 9 \mathrm{H})$.

${ }^{13} \mathbf{C}\left\{{ }^{1} \mathbf{H}\right\}$ NMR $\left(\mathbf{C D C l}_{3}, \mathbf{1 0 0} \mathbf{M H z}\right): \delta 158.5,157.5,157.1,157.0,152.2,148.8,143.0,140.2,138.0$, 137.1, 135.7, 135.1, 133.5, 133.4, 133.0, 133.0, 132.7, 132.5, 131.6, 130.8, 126.0, 124.8, 124.1, $123.4,123.3,122.7,121.4,120.6,113.8,112.8,112.4,112.3,110.6,55.4,55.4,55.2(2 \mathrm{C})$.

IR $\left(\mathrm{KBr}, \mathrm{cm}^{-1}\right):$ 3446, 3033, 2999, 1609, 1385.

HRMS (ESI) m/z: $[\mathrm{M}+\mathrm{H}]^{+}$Calcd for $\mathrm{C}_{45} \mathrm{H}_{37} \mathrm{~N}_{2} \mathrm{O}_{4}$ 669.2748; Found 669.2726. 


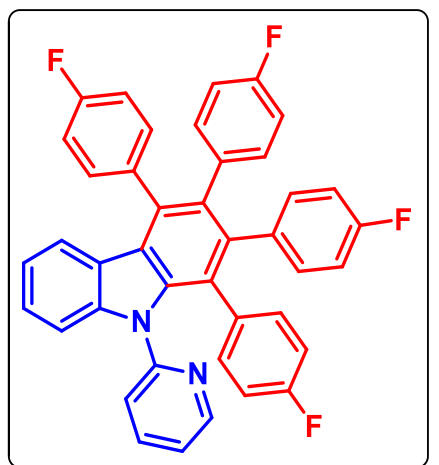

1,2,3,4-tetrakis(4-fluorophenyl)-9-(pyridin-2-yl)-9H-carbazole (5bf): was prepared according to general procedure (2.5). The crude reaction mixture was purified by column chromatography using silica gel (100-200 mesh size in 40\% EtOAc/hexane) giving $5 \mathbf{b f}$ ( $39 \mathrm{mg}$ for $0.10 \mathrm{mmol}$ scale) $63 \%$ yield.

Physical State: white solid

Melting Point: $291-297^{\circ} \mathrm{C}$

$\mathbf{R}_{\boldsymbol{f}}$-value: 0.3 (10\% EtOAc/hexane)

${ }^{1} \mathbf{H}$ NMR $\left(\mathbf{C D C l}_{3}, \mathbf{4 0 0} \mathbf{M H z}\right): \delta 8.30\left(\mathrm{dd}, J_{l}=4.8 \mathrm{~Hz}, J_{2}=1.2 \mathrm{~Hz}, 1 \mathrm{H}\right), 7.41\left(\mathrm{dt}, J_{l}=7.6 \mathrm{~Hz}, J_{2}=\right.$ $2 \mathrm{~Hz}, 1 \mathrm{H}), 7.30-7.26(\mathrm{~m}, 4 \mathrm{H}), 7.07-7.02(\mathrm{~m}, 3 \mathrm{H}), 7.00-6.96(\mathrm{~m}, 1 \mathrm{H}), 6.93(\mathrm{~d}, J=7.6 \mathrm{~Hz}, 1 \mathrm{H}), 6.81-$ $6.77(\mathrm{~m}, 5 \mathrm{H}), 6.73-6.69(\mathrm{~m}, 2 \mathrm{H}), 6.62(\mathrm{t}, J=8.8 \mathrm{~Hz}, 2 \mathrm{H}), 6.57(\mathrm{t}, J=8.8 \mathrm{~Hz}, 2 \mathrm{H}), 6.48(\mathrm{t}, J=8.8$ $\mathrm{Hz}, 2 \mathrm{H})$.

${ }^{13} \mathbf{C}\left\{{ }^{1} \mathbf{H}\right\}$ NMR $\left(\mathbf{C D C l}_{3}, \mathbf{1 0 0} \mathbf{M H z}\right): \delta 163.5,162.4(\mathrm{~d}, J=19.3 \mathrm{~Hz}), 161.0,159.9(\mathrm{~d}, J=17.3 \mathrm{~Hz})$, 151.8, 149.4, 143.2, 139.3, 138.0, 137.6, $136.3\left(\mathrm{dd}, J_{l}=15.4 \mathrm{~Hz}, J_{2}=3.5 \mathrm{~Hz}\right), 136.0(\mathrm{~d}, J=3.5$ Hz), 135.3, 134.1, 133.9 (d, $J=3.5 \mathrm{~Hz}), 133.3(\mathrm{~m}), 133.0$ (d, $J=7.8 \mathrm{~Hz}), 132.1$ (d, $J=7.9 \mathrm{~Hz})$, 126.6, 124.4, 123.6, 123.5, 123.4, 122.6, 122.2, 121.0, 115.8, 115.6, 114.5, 114.3 (m), 114.0 (m), 110.7 .

${ }^{19}$ F NMR $\left(\mathbf{C D C l}_{3}, 376 \mathbf{M H z}\right): \delta-115.0,-116.3,-116.8,-117.0$.

IR $\left(\mathrm{KBr}, \mathrm{cm}^{-1}\right): 3942,3852,3445,1604,1436,1264$.

HRMS (ESI) m/z: [M+H] ${ }^{+}$Calcd for $\mathrm{C}_{41} \mathrm{H}_{25} \mathrm{~F}_{4} \mathrm{~N}_{2}$ 621.1948; Found 621.1923.

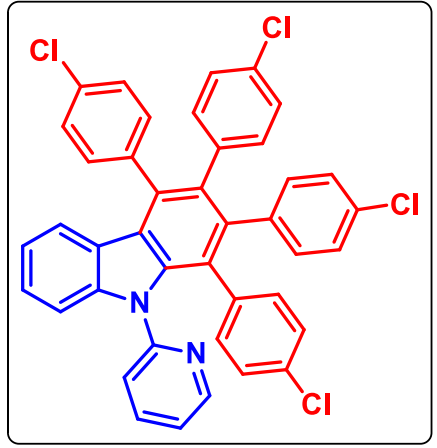

\section{1,2,3,4-tetrakis(4-chlorophenyl)-9-(pyridin-2-yl)-9H-} carbazole (5bg): was prepared according to general procedure (2.5). The crude reaction mixture was purified by column chromatography using silica gel (100-200 mesh size in 35\% EtOAc/hexane) giving $\mathbf{5 b g}$ (45 $\mathrm{mg}$ for $0.10 \mathrm{mmol} \mathrm{scale)} 66 \%$ yield.

Physical State: solid

m.p.: $252-254{ }^{\circ} \mathrm{C} \quad \mathbf{R}_{f}$-value: 0.4 (20\% EtOAc/hexane)

${ }^{1} \mathbf{H}$ NMR $\left(\mathbf{C D C l}_{3}, 400 \mathrm{MHz}\right): \delta 8.29\left(\mathrm{dd}, J_{l}=4.8 \mathrm{~Hz}, J_{2}=1.2 \mathrm{~Hz}, 1 \mathrm{H}\right), 7.42\left(\mathrm{dt}, J_{1}=7.6 \mathrm{~Hz}, J_{2}=\right.$ $2.0 \mathrm{~Hz}, 1 \mathrm{H}), 7.34-7.32(\mathrm{~m}, 2 \mathrm{H}), 7.29\left(\mathrm{dd}, J_{l}=6.8 \mathrm{~Hz}, J_{2}=1.2 \mathrm{~Hz}, 1 \mathrm{H}\right), 7.26-7.22(\mathrm{~m}, 4 \mathrm{H}), 7.08-$ $7.05(\mathrm{~m}, 1 \mathrm{H}), 7.01-6.97(\mathrm{~m}, 1 \mathrm{H}), 6.92-6.85(\mathrm{~m}, 5 \mathrm{H}), 6.80(\mathrm{~d}, J=8.0 \mathrm{~Hz}, 1 \mathrm{H}), 6.77-6.74(\mathrm{~m}, 5 \mathrm{H})$ 6.70-6.67 (m, 2H).

${ }^{13} \mathbf{C}\left\{{ }^{1} \mathbf{H}\right\}$ NMR $\left(\mathbf{C D C l}_{3}, \mathbf{1 0 0} \mathbf{M H z}\right): \delta 151.6,149.5,143.1,138.6,138.6,138.5,138.3,138.0,137.7$, 136.2 , 135.0, 133.5, 133.4, 133.1, 133.0, 132.7, 132.3, 132.1, 132.0, 131.8, 129.0, 127.6, 127.5, $127.5,126.8,124.1,123.4,123.3,122.6,122.1,121.1,110.7$.

IR $\left(\mathrm{KBr}, \mathrm{cm}^{-1}\right): 3440,2349,1634,1435,1014$.

HRMS (ESI) m/z: $[\mathrm{M}+\mathrm{H}]^{+}$Calcd for $\mathrm{C}_{41} \mathrm{H}_{25} \mathrm{Cl}_{4} \mathrm{~N}_{2}$ 685.0766; Found 685.0729. 


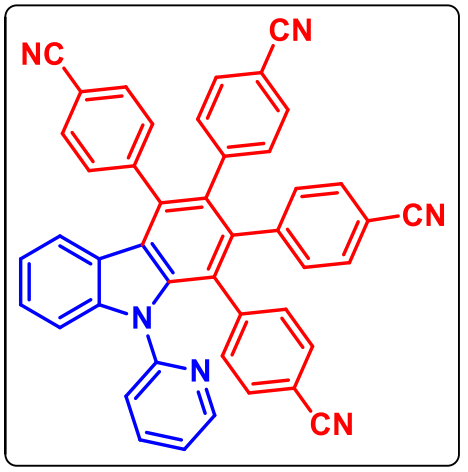

4,4',4",4"'-(9-(pyridin-2-yl)-9H-carbazole-1,2,3,4-tetrayl) tetrabenzonitrile (5bi): was prepared according to general procedure (2.5). The crude reaction mixture was purified by column chromatography using silica gel (100-200 mesh size in $20 \%$ EtOAc/hexane) giving 5 bi (48 $\mathrm{mg}$ for $0.10 \mathrm{mmol}$ scale) $74 \%$ yield.

Physical State: light pink solid

m.p.: $280-285^{\circ} \mathrm{C}$

$\mathbf{R}_{f}$-value: 0.3 (10\% EtOAc/hexane)

${ }^{1} \mathbf{H}$ NMR (CDCl 3 , $\left.400 \mathrm{MHz}\right): \delta 8.25\left(\mathrm{dd}, \boldsymbol{J}_{1}=4.4 \mathrm{~Hz}, J_{2}=1.2 \mathrm{~Hz}, 1 \mathrm{H}\right), 7.70-7.65(\mathrm{~m}, 3 \mathrm{H}), 7.55-$ $7.48(\mathrm{~m}, 2 \mathrm{H}), 7.45-7.43(\mathrm{~m}, 2 \mathrm{H}), 7.38(\mathrm{t}, J=8.4 \mathrm{~Hz}, 1 \mathrm{H}), 7.25(\mathrm{~d}, J=3.2 \mathrm{~Hz}, 1 \mathrm{H}), 7.20(\mathrm{~d}, J=8.0$ $\mathrm{Hz}, 2 \mathrm{H}), 7.12-7.09(\mathrm{~m}, 3 \mathrm{H}), 7.06-7.03(\mathrm{~m}, 2 \mathrm{H}), 6.97-6.93(\mathrm{~m}, 4 \mathrm{H}), 6.87(\mathrm{~d}, J=8.0 \mathrm{~Hz}, 2 \mathrm{H}), 6.70$ $(\mathrm{d}, J=8.0 \mathrm{~Hz}, 1 \mathrm{H})$.

${ }^{13} \mathbf{C}\left\{{ }^{1} \mathbf{H}\right\}$ NMR (CDCl $\left.\mathbf{3}, \mathbf{1 0 0} \mathbf{M H z}\right): \delta 151.1,150.1,144.3,144.1,144.0,143.3,142.2,138.1,137.8$, 137.4, 134.7, 132.8, 132.5, 132.4 (2C), 132.3 (3C), 132.1, 132.1, 131.5, 131.4, 131.3, 128.9, 128.8, $127.8,123.9,123.7,123.3,123.0,122.5,122.4,121.9,118.7,118.6,118.5$ (2C), 112.4, $111.0(2 \mathrm{C})$, 110.8 .

IR $\left(\mathrm{KBr}, \mathrm{cm}^{-1}\right): 3441,2922,1393,1184$.

HRMS (ESI) m/z: [M+H] $]^{+}$Calcd for $\mathrm{C}_{45} \mathrm{H}_{25} \mathrm{~N}_{6}, 649.2135$; Found 649.2106.

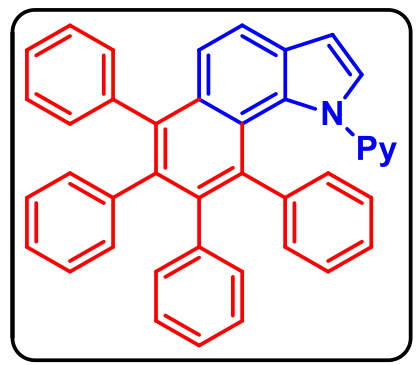

6,7,8,9-tetraphenyl-1-(pyridin-2-yl)-1H-benzo[g]indole (6): was prepared according to general procedure (5.1). The crude reaction mixture was purified by column chromatography using silica gel (100-200 mesh size in 15\% EtOAc/hexane) giving 6 (51 mg for 0.10 mmol scale) $93 \%$ yield.

Physical State: white solid m.p.: $275-280{ }^{\circ} \mathrm{C}$

$\mathbf{R}_{f}$-value: $0.2(5 \% \mathrm{EtOAc/hexane)}$

${ }^{1}$ H NMR (CDCl, 400 MHz): $\delta 8.05(\mathrm{~d}, J=4.0 \mathrm{~Hz}, 1 \mathrm{H}), 7.63(\mathrm{~d}, J=8.4 \mathrm{~Hz}, 1 \mathrm{H}), 7.40(\mathrm{~d}, J=8.8$ $\mathrm{Hz}, 1 \mathrm{H}), 7.34(\mathrm{t}, J=8.0 \mathrm{~Hz}, 1 \mathrm{H}), 7.25-7.19(\mathrm{~m}, 6 \mathrm{H}), 6.91(\mathrm{t}, J=6.4 \mathrm{~Hz}, 1 \mathrm{H}), 6.84-6.57(\mathrm{~m}, 17 \mathrm{H})$.

${ }^{13} \mathbf{C}\left\{{ }^{1} \mathbf{H}\right\}$ NMR $\left(\mathbf{C D C l}_{3}, \mathbf{1 0 0} \mathbf{M H z}\right): \delta 154.4,148.7,141.3,141.1,141.0,140.9,140.6,139.6,139.5$, 137.9, 134.6, 131.84, 131.8, 131.7 (2C), 131.3, 130.1, 127.7, 126.9, 126.7 (2C), 126.6, 125.5, 125.4, $125.3,125.2,123.0,121.7,120.9,120.7,117.2,106.6$.

IR $\left(\mathrm{KBr}, \mathrm{cm}^{-1}\right):$ 3439, 2986, 1601, 1264.

HRMS (ESI) m/z: [M+H] $]^{+}$Calcd for $\mathrm{C}_{41} \mathrm{H}_{29} \mathrm{~N}_{2}$ 549.2325; Found 549.2292. 


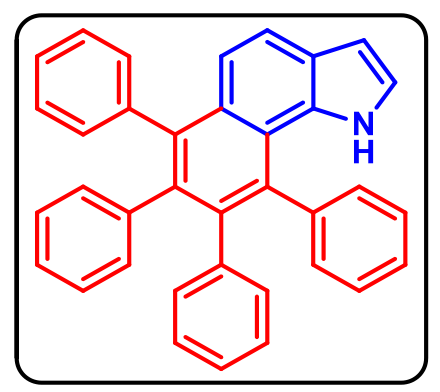

6,7,8,9-Tetraphenyl-1H-benzo[g]indole (7): was prepared according to general procedure (5.2). The crude reaction mixture was purified by column chromatography using silica gel (100-200 mesh size in $10 \% \mathrm{EtOAc} /$ hexane) giving 7 (17 mg for $0.05 \mathrm{mmol}$ scale) $72 \%$ yield.

Physical State: white solid

m.p.: $270-280{ }^{\circ} \mathrm{C}$

$\mathbf{R}_{\boldsymbol{f}}$-value: 0.7 (10\% EtOAc/hexane)

${ }^{1} \mathbf{H}$ NMR $\left(\mathbf{C D C l}_{3}, 400 \mathrm{MHz}\right): \delta 7.65(\mathrm{~d}, J=8.8 \mathrm{~Hz}, 1 \mathrm{H}), 7.36-7.32(\mathrm{~m}, 7 \mathrm{H}), 7.24(\mathrm{~d}, J=4.4 \mathrm{~Hz}$, $4 \mathrm{H}), 7.20-7.17(\mathrm{~m}, 1 \mathrm{H}), 6.87-6.80(\mathrm{~m}, 11 \mathrm{H}), 6.55(\mathrm{~s}, 1 \mathrm{H})$.

${ }^{13} \mathbf{C}\left\{{ }^{1} \mathbf{H}\right\}$ NMR $\left(\mathbf{C D C l}_{\mathbf{3}}, \mathbf{1 7 6} \mathbf{M H z}\right): \delta 141.9,141.1,141.0,140.6,139.7,139.2,137.3,134.2,131.8$, 131.7, 131.6, 131.3, 130.8, 129.5, 128.9, 127.8 (2C), 126.8 (2C), 126.6, 125.6, 125.5, 125.4, 122.7, $121.4,120.6,120.3,103.3$.

IR $\left(\mathrm{KBr}, \mathrm{cm}^{-1}\right): 3456,3055,1366,1264$.

HRMS (ESI) m/z: [M+H] $]^{+}$Calcd for $\mathrm{C}_{36} \mathrm{H}_{26} \mathrm{~N}$ 472.2060; Found 472.2060.

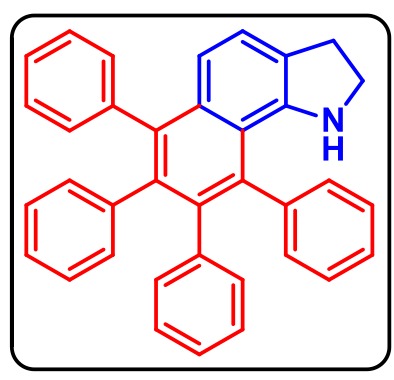

6,7,8,9-tetraphenyl-2,3-dihydro-1H-benzo[g]indole (8): was prepared according to general procedure (5.3). The crude reaction mixture was purified by column chromatography using silica gel (100-

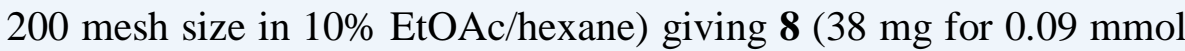
scale) $88 \%$ yield.

Physical State: yellow solid

m.p.: $180-185^{\circ} \mathrm{C}$

$\mathbf{R}_{f}$-value: 0.4 (5\% EtOAc/hexane)

${ }^{1} \mathbf{H}$ NMR (CDCl 3 , 400 MHz): $\delta 7.27-7.12(\mathrm{~m}, 10 \mathrm{H}), 6.99(\mathrm{~d}, J=8.4 \mathrm{~Hz}, 1 \mathrm{H}), 6.87-6.75(\mathrm{~m}, 11 \mathrm{H})$, $3.33(\mathrm{t}, J=8.4 \mathrm{~Hz}, 2 \mathrm{H}), 3.02(\mathrm{t}, J=8.8 \mathrm{~Hz}, 2 \mathrm{H})$.

${ }^{13} \mathbf{C}\left\{{ }^{1} \mathbf{H}\right\}$ NMR (CDCl $\left.\mathbf{3}, \mathbf{1 0 0} \mathrm{MHz}\right): \delta 148.5,141.9,141.0,140.9$ (2C), 140.7, 140.6, 139.2, 138.8, $138.3,136.0,133.2,131.7,131.5,131.0,127.7$ (2C), 127.2, 126.9, 126.8, 126.7, 126.5, 125.5, $125.1,124.0,119.0,118.4,48.2,30.2$.

IR $\left(\mathrm{KBr}, \mathrm{cm}^{-1}\right):$ 3053, 2986, 1420, 1264.

HRMS (ESI) m/z: $[\mathrm{M}+\mathrm{H}]^{+}$Calcd for $\mathrm{C}_{36} \mathrm{H}_{28} \mathrm{~N}$ 474.2216; Found 474.2184. 


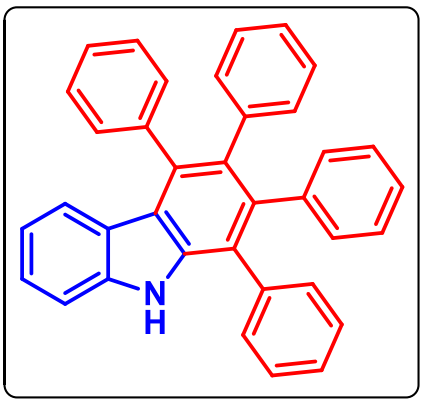

1,2,3,4-tetraphenyl-9H-carbazole (9): was prepared according to general procedure (5.4). The crude reaction mixture was purified by column chromatography using silica gel (100-200 mesh size in $35 \%$ EtOAc/hexane) giving 9 (39 $\mathrm{mg}$ for $0.10 \mathrm{mmol} \mathrm{scale)} 82 \%$ yield.

Physical State: yellow solid

m.p.: $232-234{ }^{\circ} \mathrm{C}$

$\mathbf{R}_{\boldsymbol{f}}$-value: 0.3 (20\% EtOAc/hexane)

${ }^{1}$ H NMR (CDCl 3 , 400 MHz): $\delta 8.08(\mathrm{~s}, 1 \mathrm{H}), 7.33-7.28(\mathrm{~m}, 11 \mathrm{H}), 6.90-6.84(\mathrm{~m}, 13 \mathrm{H})$.

${ }^{13} \mathbf{C}\left\{{ }^{1} \mathbf{H}\right\}$ NMR $\left(\mathbf{C D C l}_{3}, \mathbf{1 7 6} \mathbf{M H z}\right): \delta 140.6,140.4,140.3,138.5,138.0,137.7,135.8,133.6,132.4$, 132.0, 130.8, 130.6, 128.8, 128.2, 127.2, 127.0, 127.0, 126.8, 125.9, 125.7, 125.4, 123.9, 123.6, $122.8,121.0,119.5,110.6$.

IR $\left(\mathrm{KBr}, \mathrm{cm}^{-1}\right):$ 3445, 3053, 1436, 1274.

HRMS (ESI) m/z: $[\mathrm{M}+\mathrm{Na}]^{+}$Calcd for $\mathrm{C}_{36} \mathrm{H}_{25} \mathrm{NNa}$ 494.1879; Found 494.1835.

(b) Experimental characterization data for internal alkynes: ${ }^{5}$

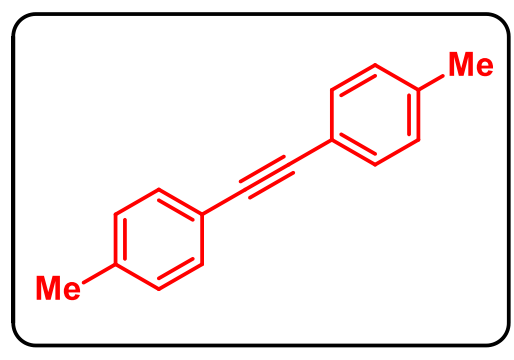

1,2-di-p-tolylethyne (2b): was prepared according to reported procedure (2.3). The crude reaction mixture was purified by column chromatography using silica gel (100-200 mesh size in 4\% EtOAc/hexane) giving $\mathbf{2 b}$ (197 $\mathrm{mg}$ for $1.0 \mathrm{mmol} \mathrm{scale}$ ) $96 \%$ yield.

Physical State: off white solid $\mathbf{R}_{f}$-value: 0.6 (2\% EtOAc/hexane)

${ }^{1} \mathbf{H}$ NMR (CDCl $\left.3,400 \mathrm{MHz}\right): \delta 7.41(\mathrm{~d}, J=8.0 \mathrm{~Hz}, 4 \mathrm{H}), 7.14(\mathrm{~d}, J=8.0 \mathrm{~Hz}, 4 \mathrm{H}), 2.36(\mathrm{~s}, 6 \mathrm{H})$.

${ }^{13} \mathbf{C}\left\{{ }^{1} \mathbf{H}\right\}$ NMR $\left(\mathbf{C D C l}_{3}, \mathbf{1 0 0} \mathbf{M H z}\right): \delta 138.5,131.7,129.4,120.6,89.2,21.8$. 


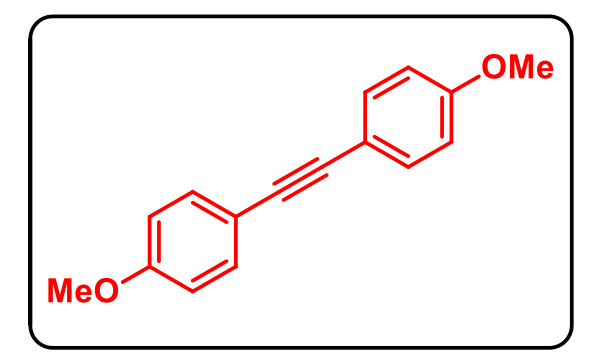

1,2-bis(4-methoxyphenyl)ethyne (2c): was prepared according to reported procedure (2.3). The crude reaction mixture was purified by column chromatography using silica gel (100-200 mesh size in 5\% EtOAc/hexane) giving 2b (195 $\mathrm{mg}$ for $1.0 \mathrm{mmol}$ scale) $81 \%$ yield.

Physical State: yellow solid

$\mathbf{R}_{\boldsymbol{f}}$-value: 0.5 (2\% EtOAc/hexane)

${ }^{1}$ H NMR (CDCl, 400 MHz): $\delta 7.44(\mathrm{~d}, J=8.4 \mathrm{~Hz}, 4 \mathrm{H}), 6.86(\mathrm{~d}, J=8.4 \mathrm{~Hz}, 4 \mathrm{H}), 3.80$ (s, 6H).

${ }^{13} \mathbf{C}\left\{{ }^{1} \mathbf{H}\right\}$ NMR $\left(\mathbf{C D C l}_{3}, \mathbf{1 7 6} \mathbf{M H z}\right): \delta 159.7,133.2,116.0,114.3,88.2,55.6$.

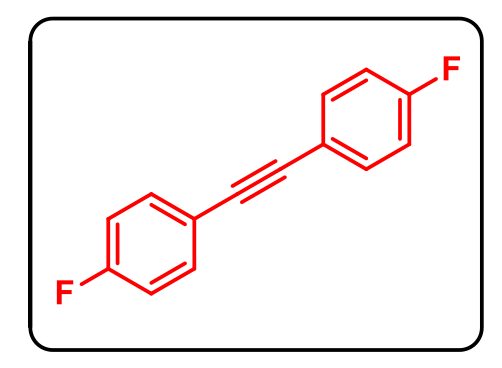

1,2-bis(4-fluorophenyl)ethyne (2d): was prepared according to reported procedure (2.3). The crude reaction mixture was purified by column chromatography using silica gel (100-200 mesh size in 4\% EtOAc/hexane) giving $\mathbf{2 b}$ (203 mg for $1.0 \mathrm{mmol} \mathrm{scale}$ ) 95\% yield.

Physical State: yellow solid

$\mathbf{R}_{\boldsymbol{f}}$-value: 0.7 (2\% EtOAc/hexane)

${ }^{1} \mathbf{H}$ NMR (CDCl 3 , 400 MHz): $\delta$ 7.49-7.45 (m, 4H), 7.04-7.00 (m, 4H).

${ }^{13} \mathbf{C}\left\{{ }^{1} \mathbf{H}\right\}$ NMR $\left(\mathbf{C D C l}_{3}, \mathbf{1 0 0} \mathbf{M H z}\right): \delta 162.8(\mathrm{~d}, J=240 \mathrm{~Hz}), 133.7(\mathrm{~d}, J=9.0), 119.5(\mathrm{~d}, J=3.4 \mathrm{~Hz})$, $115.9(\mathrm{~d}, J=21.9 \mathrm{~Hz}), 88.2$.

${ }^{19}$ F NMR $\left(\mathbf{C D C l}_{3}, 376 \mathrm{MHz}\right): \delta-110.6$,

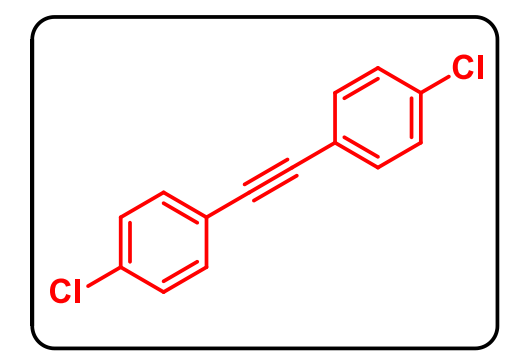

1,2-bis(4-chlorophenyl)ethyne (2e): was prepared according to reported procedure $(\mathbf{2 . 3})$. The crude reaction mixture was purified by column chromatography using silica gel (100-200 mesh size in $4 \%$ EtOAc/hexane) giving $\mathbf{2 b}$ (217 mg for $1.0 \mathrm{mmol} \mathrm{scale}) 87 \%$ yield.

Physical State: white solid

$\mathbf{R}_{\boldsymbol{f}}$-value: 0.5 (2\% EtOAc/hexane)

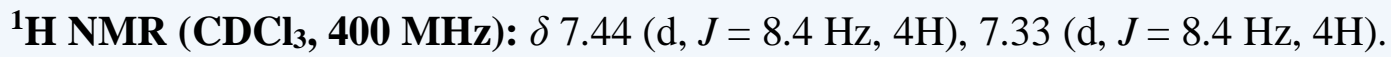

${ }^{13} \mathbf{C}\left\{{ }^{1} \mathbf{H}\right\}$ NMR $\left(\mathbf{C D C l}_{3}, \mathbf{1 0 0} \mathbf{M H z}\right): \delta 134.8,133.1,129.1,121.7,89.5$. 


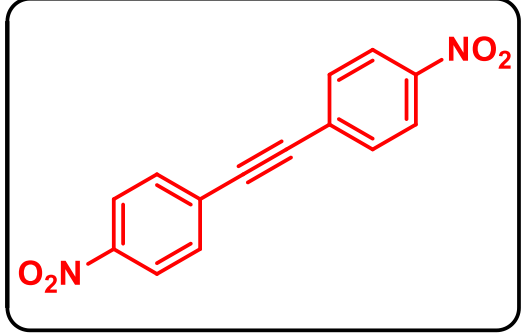

1,2-bis(4-nitrophenyl)ethyne (2f): was prepared according to reported procedure (2.3). The crude reaction mixture was purified by column chromatography using silica gel (100-200 mesh size in $20 \%$ EtOAc/hexane) giving $\mathbf{2 b}$ (134 $\mathrm{mg}$ for $1.0 \mathrm{mmol} \mathrm{scale})$ $49 \%$ yield.

Physical State: yellow solid

$\mathbf{R}_{f}$-value: 0.4 (15\% EtOAc/hexane)

${ }^{1} \mathbf{H}$ NMR (CDCl $\left.3,400 \mathrm{MHz}\right): \delta 8.26(\mathrm{~d}, J=8.8 \mathrm{~Hz}, 4 \mathrm{H}), 7.72(\mathrm{~d}, J=8.8 \mathrm{~Hz}, 4 \mathrm{H})$.

${ }^{13} \mathbf{C}\left\{{ }^{1} \mathbf{H}\right\}$ NMR (CDCl $\left.\mathbf{3}, \mathbf{1 7 6} \mathbf{M H z}\right): \delta 147.9,132.9,124.1,92.3$.

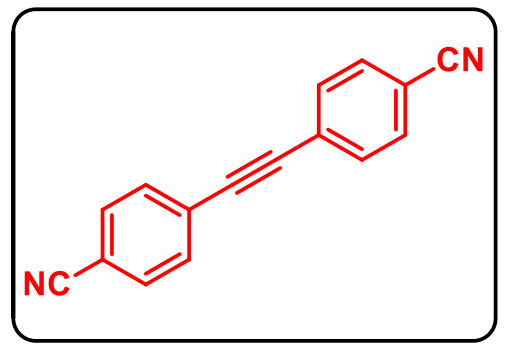

4,4'-(ethyne-1,2-diyl)dibenzonitrile (2i): was prepared according to reported procedure $(\mathbf{2 . 3})$. The crude reaction mixture was purified by column chromatography using silica gel (100-200 mesh size in $20 \%$ EtOAc/hexane) giving $\mathbf{2 b}$ (121 $\mathrm{mg}$ for $1.0 \mathrm{mmol} \mathrm{scale})$ $52 \%$ yield.

Physical State: yellow solid

$\mathbf{R}_{\boldsymbol{f}}$-value: 0.3 (10\% EtOAc/hexane)

${ }^{1} \mathbf{H}$ NMR (CDCl 3 , $\left.700 \mathrm{MHz}\right): \delta 7.67(\mathrm{~d}, J=8.4 \mathrm{~Hz}, 4 \mathrm{H}), 7.63(\mathrm{~d}, J=8.4 \mathrm{~Hz}, 4 \mathrm{H})$.

${ }^{13} \mathbf{C}\left\{{ }^{1} \mathbf{H}\right\}$ NMR $\left(\mathbf{C D C l}_{3}, \mathbf{1 7 6} \mathbf{M H z}\right): \delta 132.6,132.5,127.4,118.6,112.7,91.8$. 
9. Copies of NMR spectra of the products and post-functionalized adducts:

${ }^{1} \mathrm{H}$ NMR of 3aa $\left(400 \mathrm{MHz}, \mathrm{CDCl}_{3}\right)$

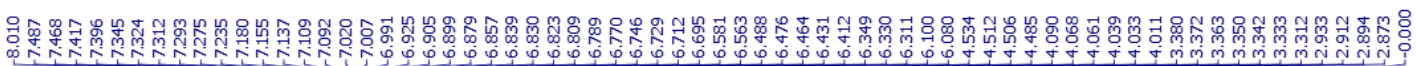

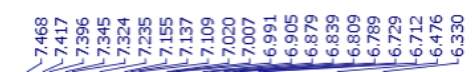
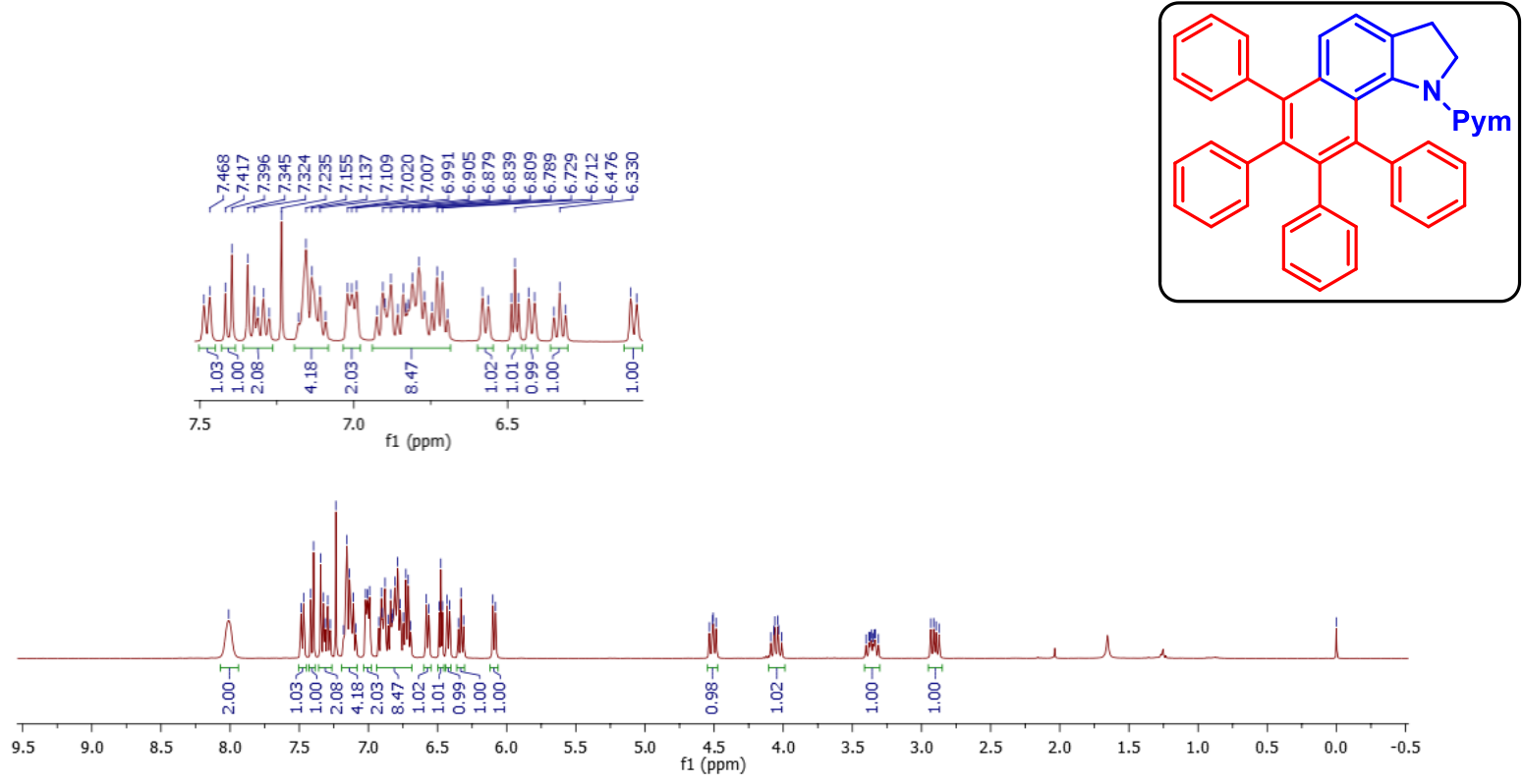

${ }^{13} \mathrm{C}\left\{{ }^{1} \mathrm{H}\right\}$ NMR of $3 \mathrm{aa}\left(100 \mathrm{MHz}, \mathrm{CDCl}_{3}\right)$

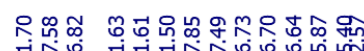

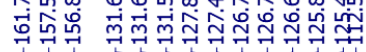

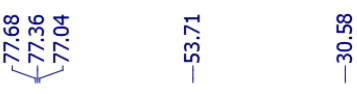
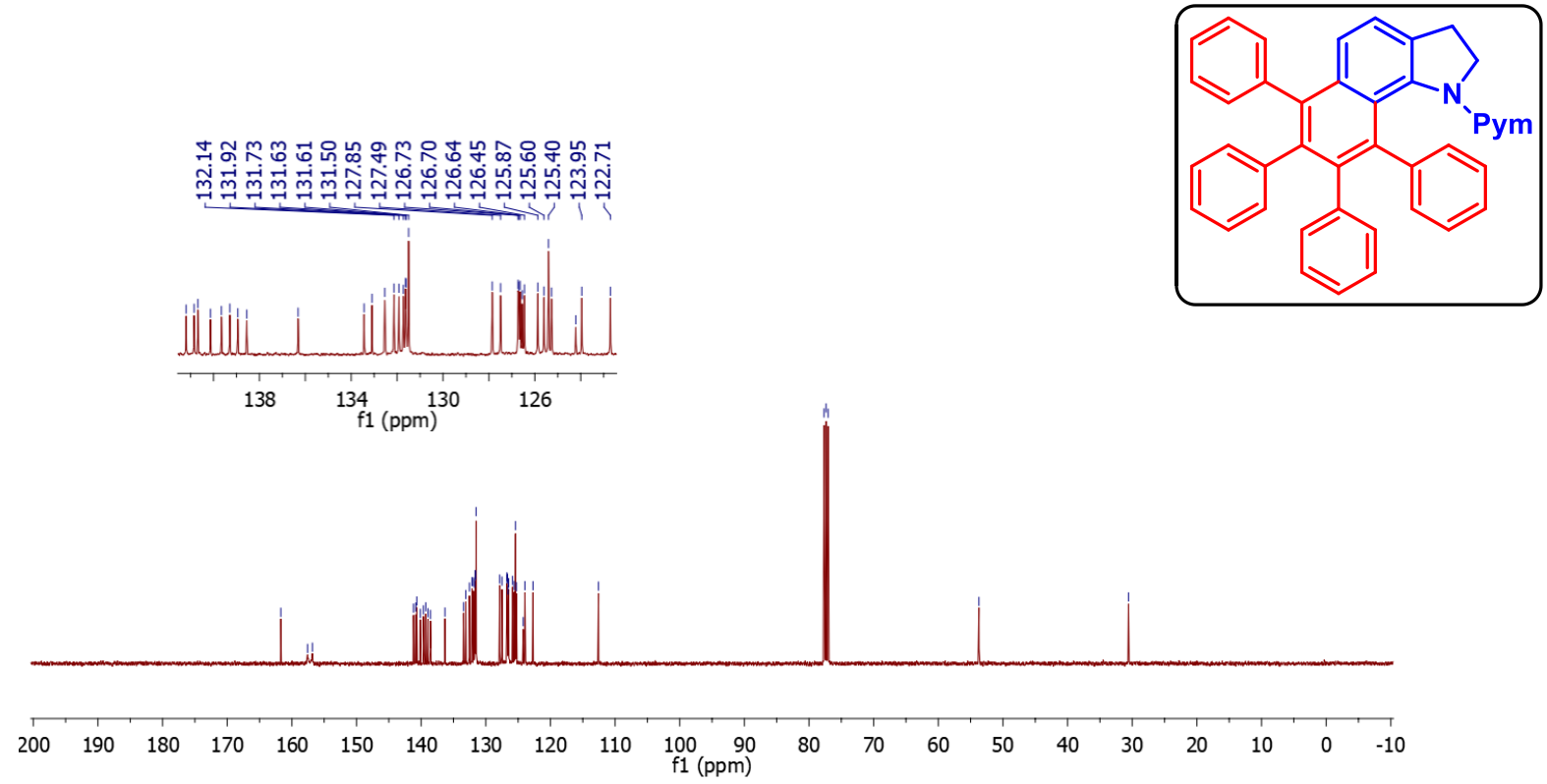

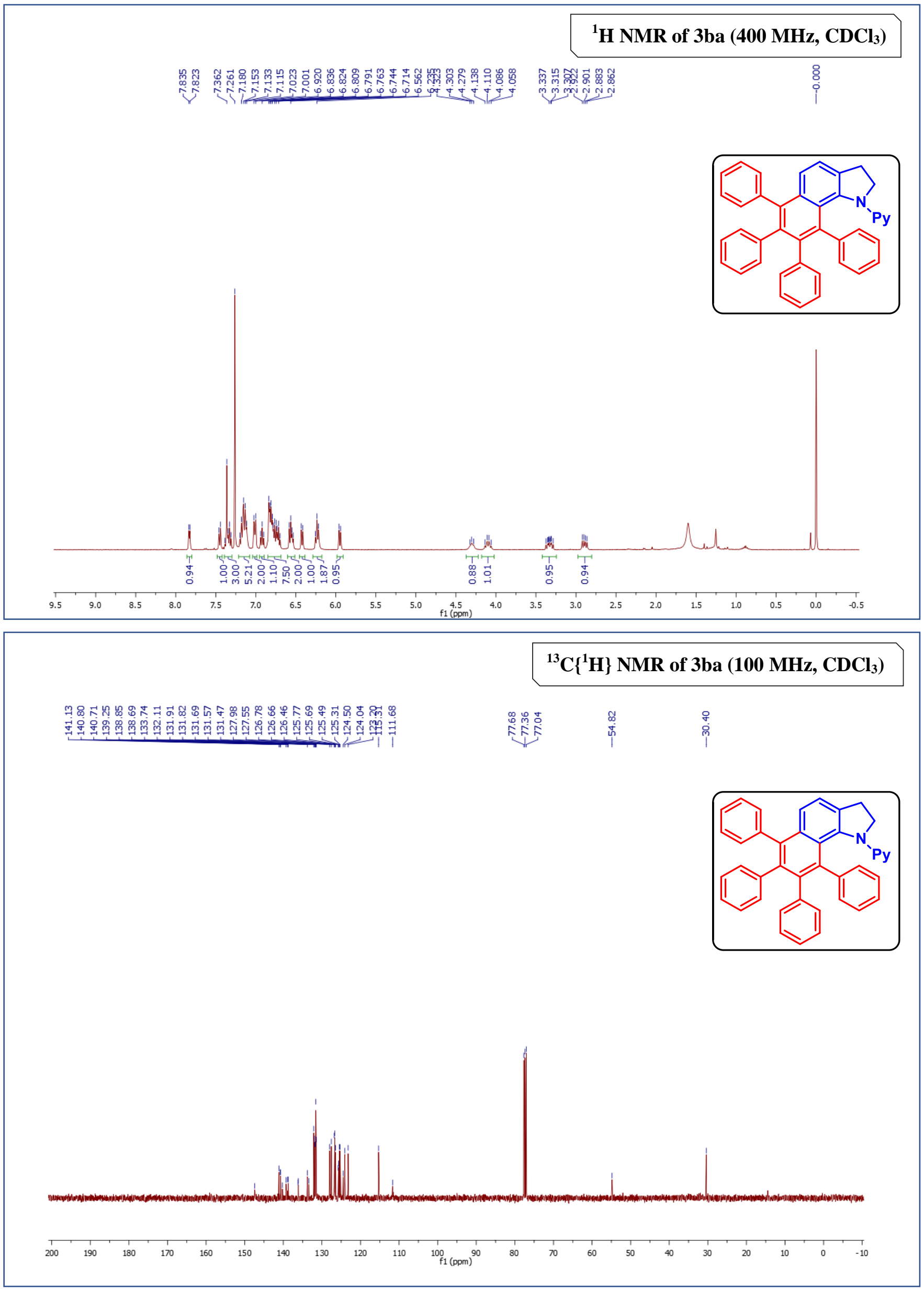


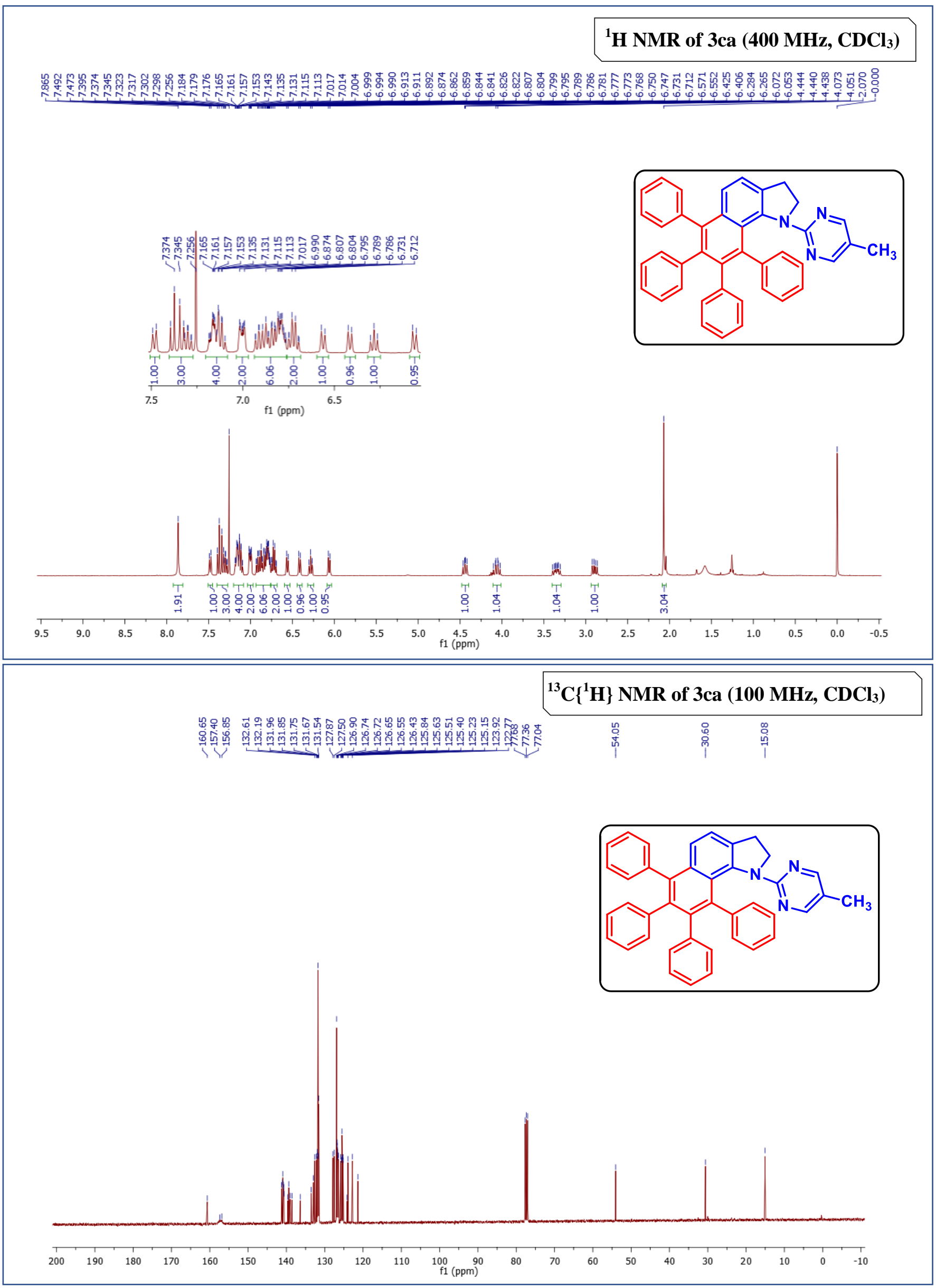




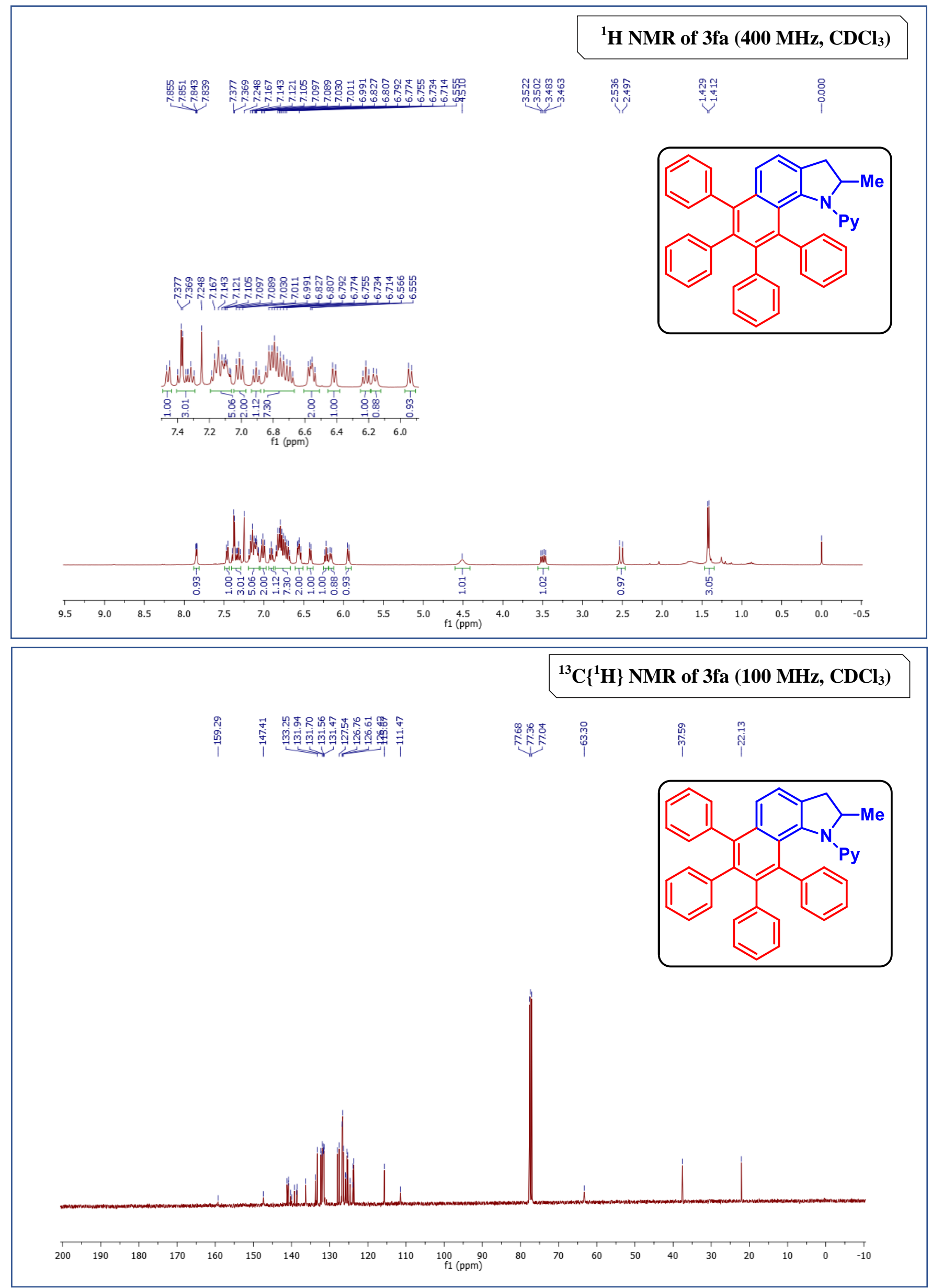




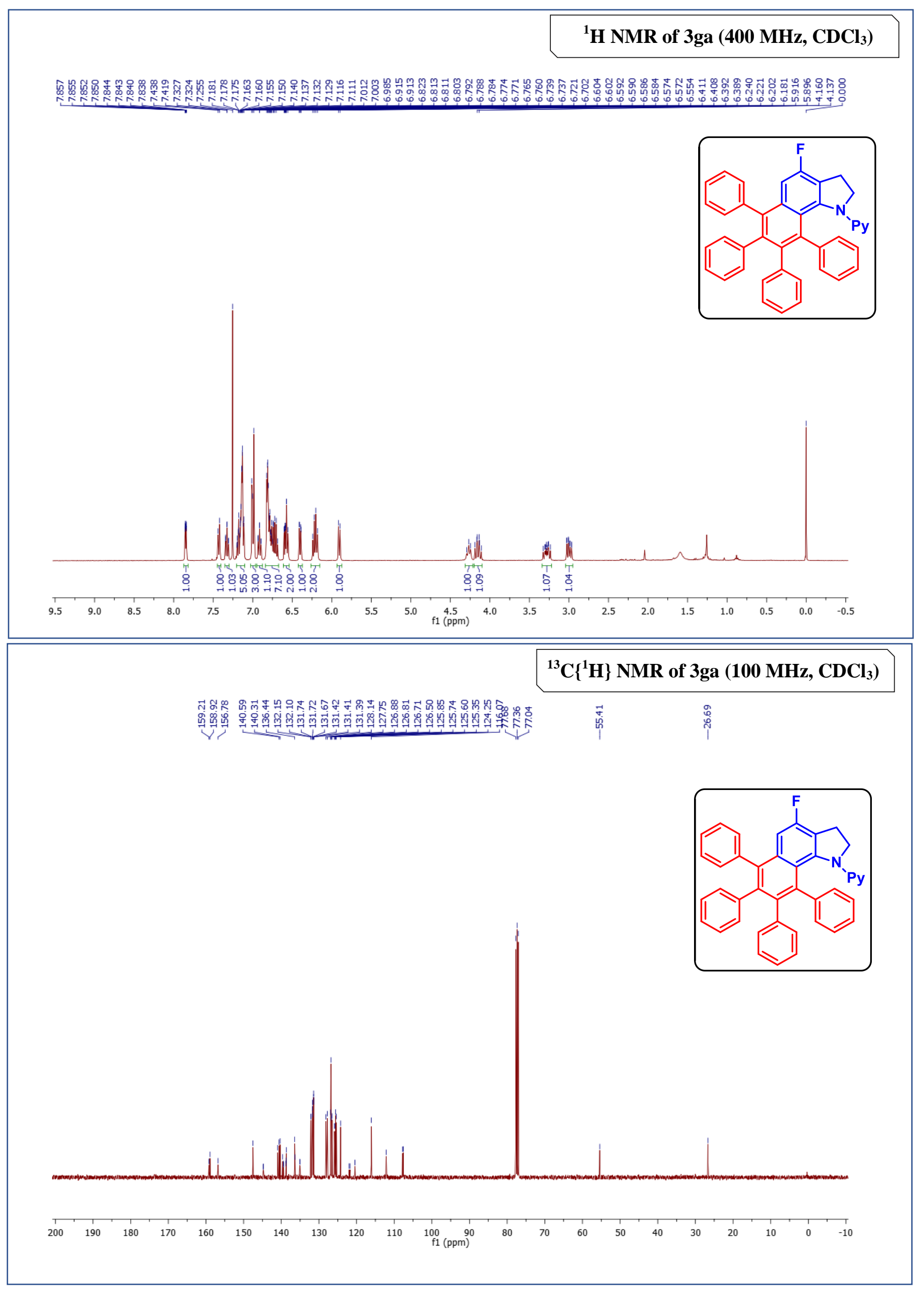




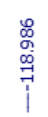

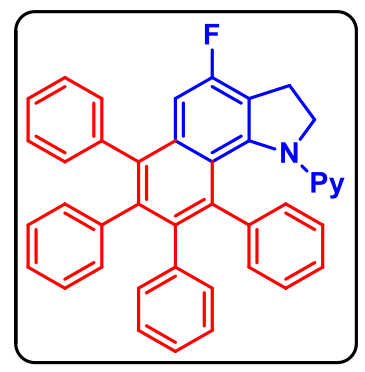

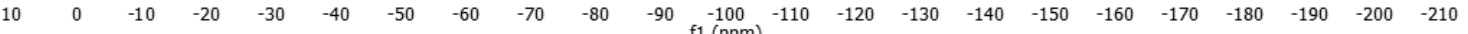




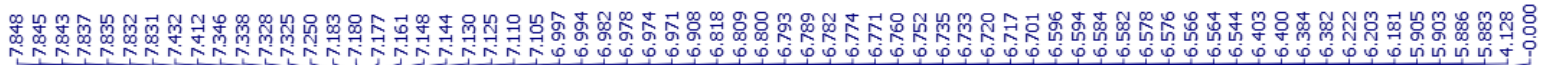
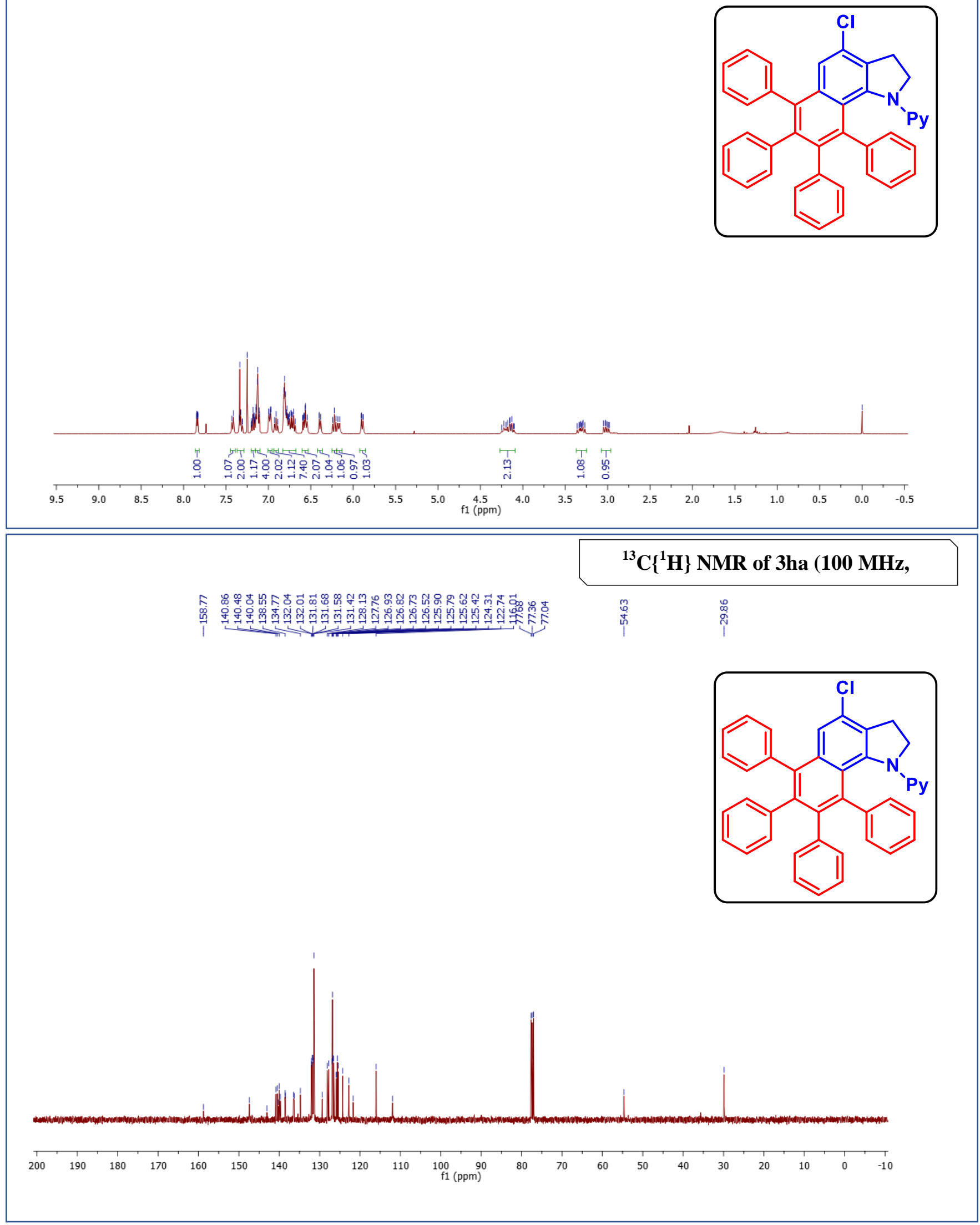


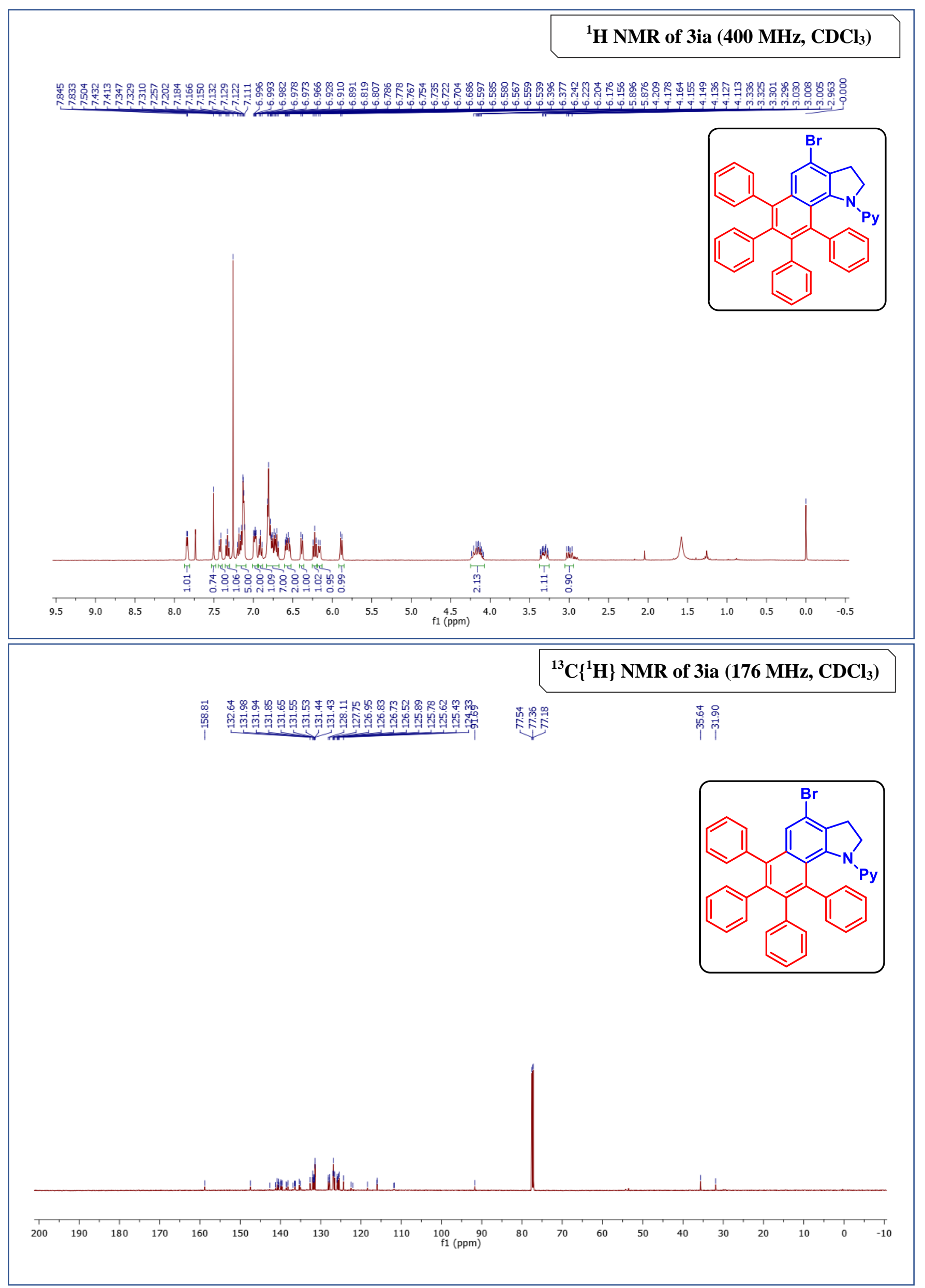




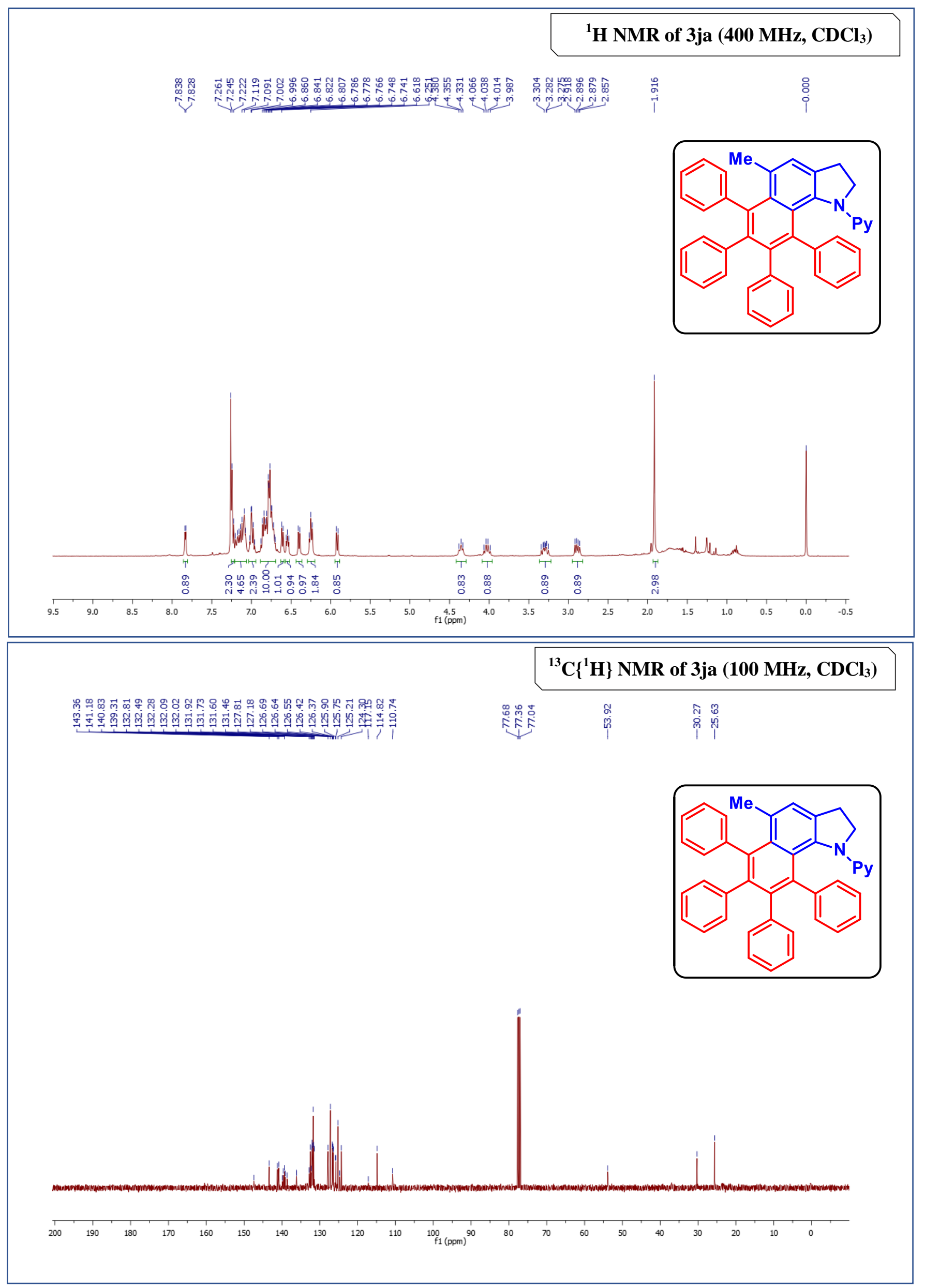




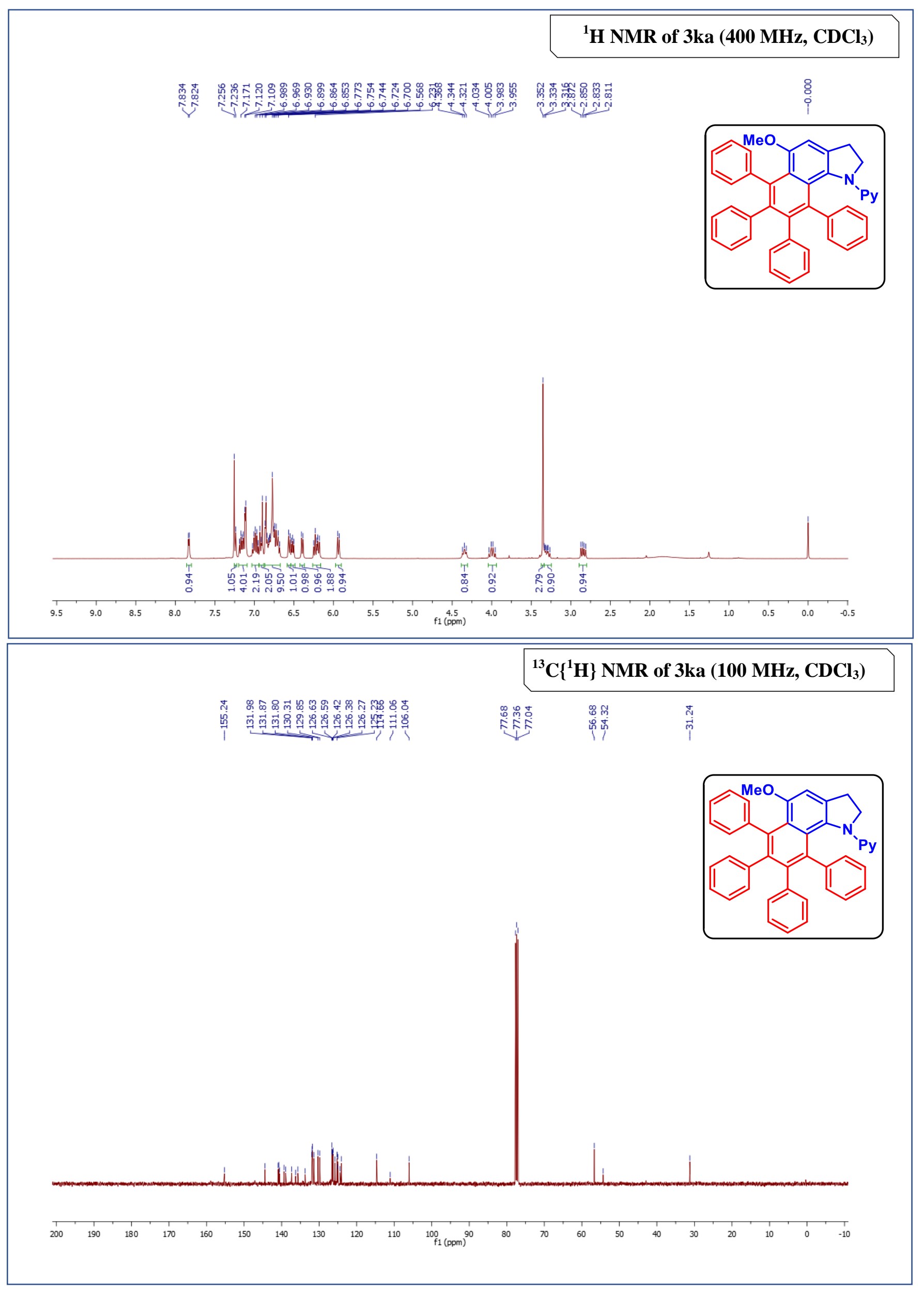




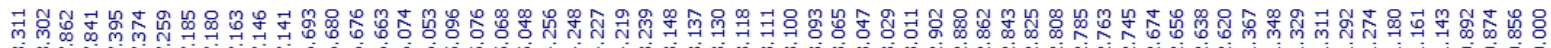

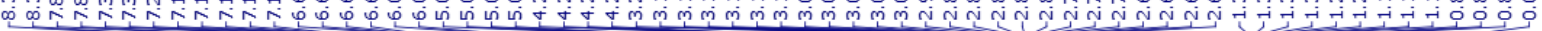

的品击

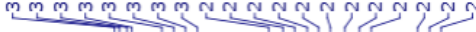
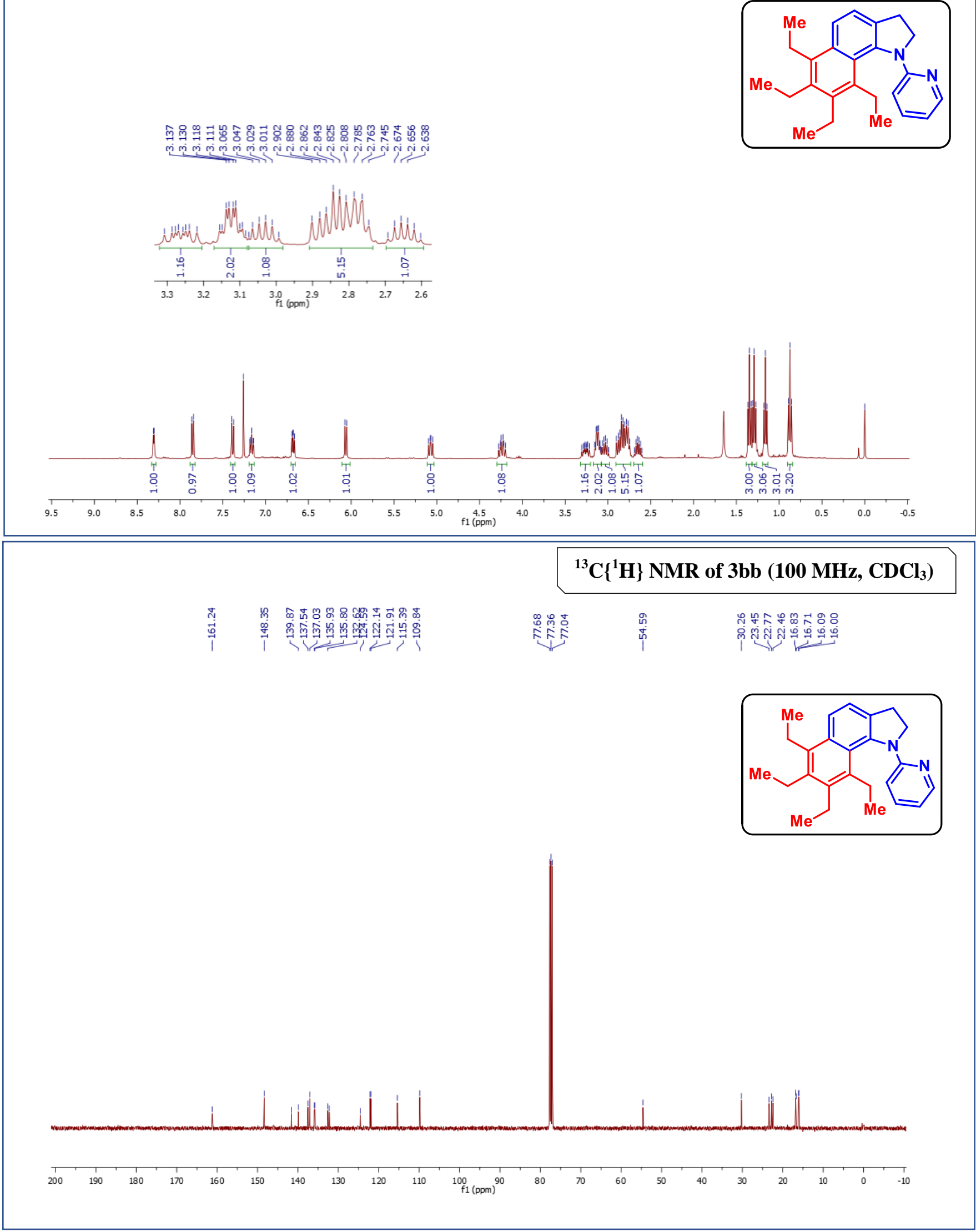


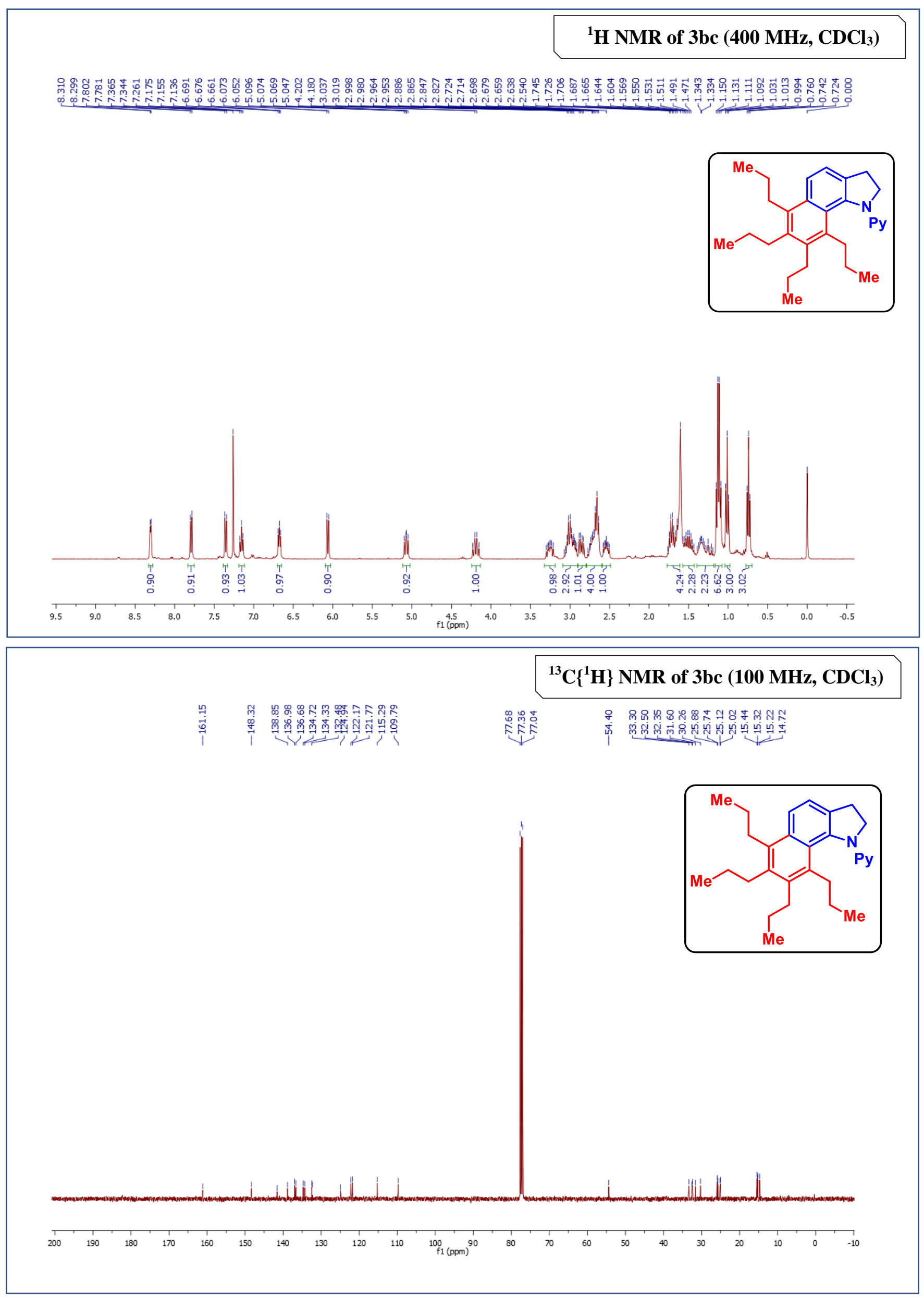




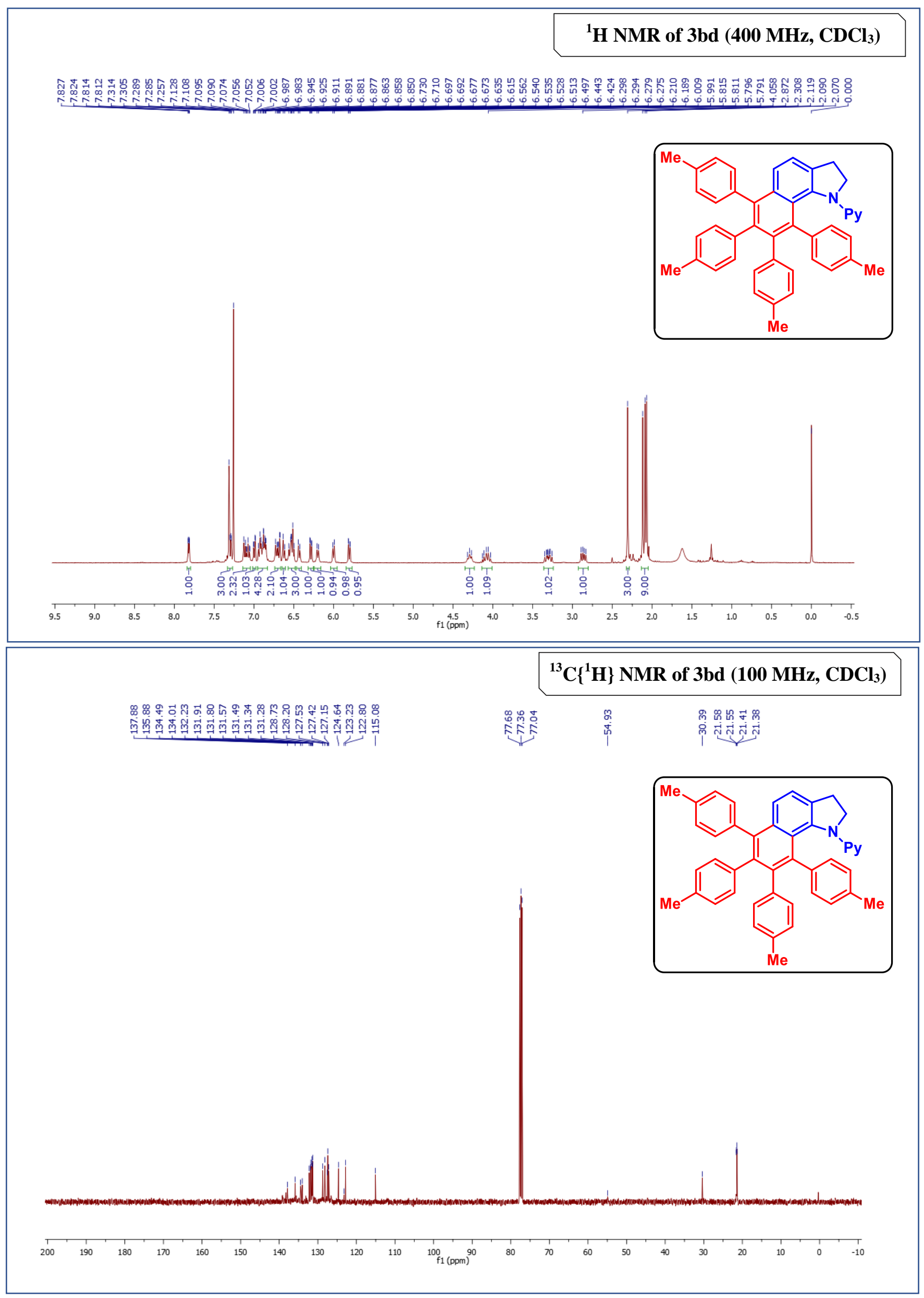




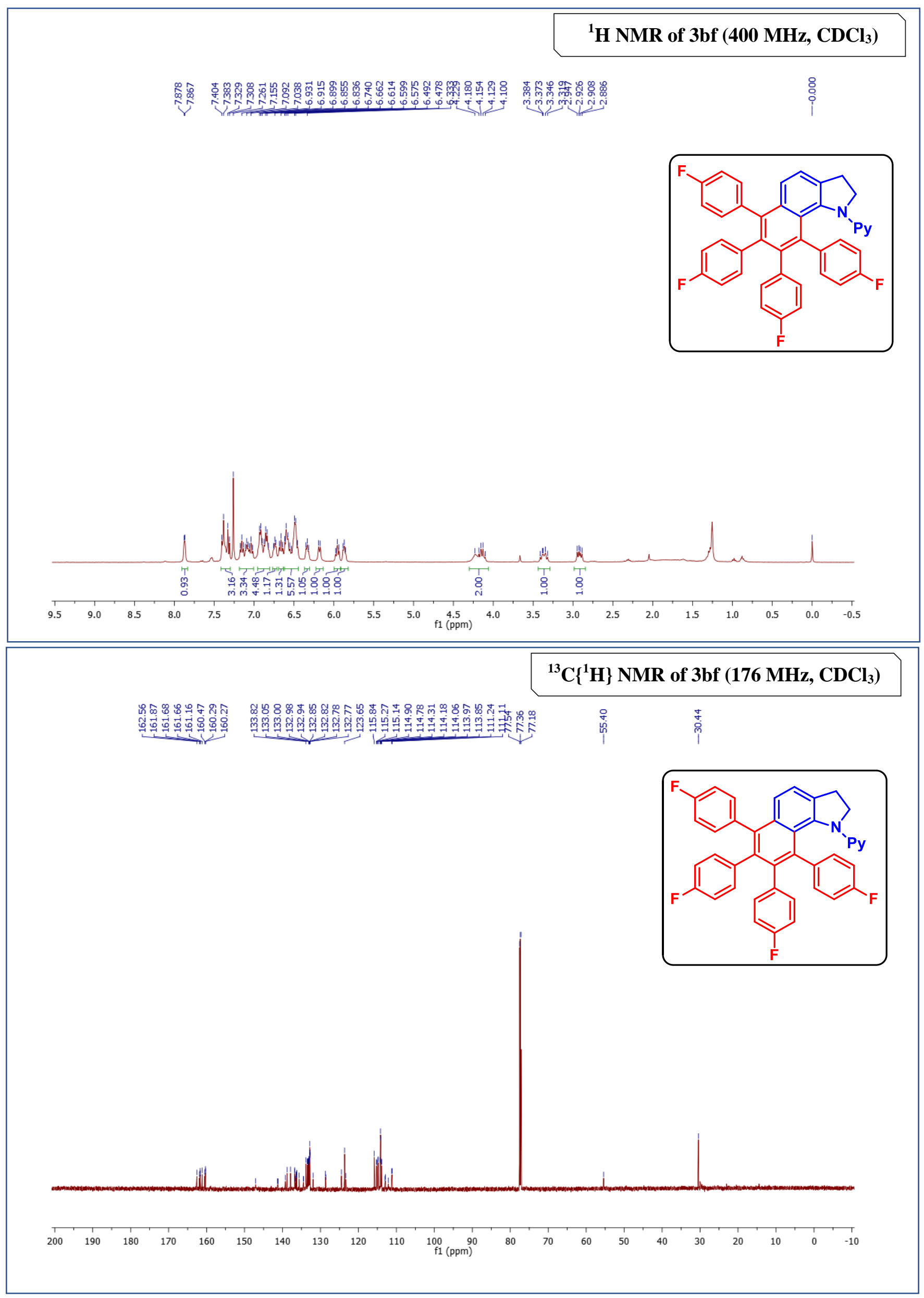




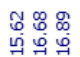

மำ
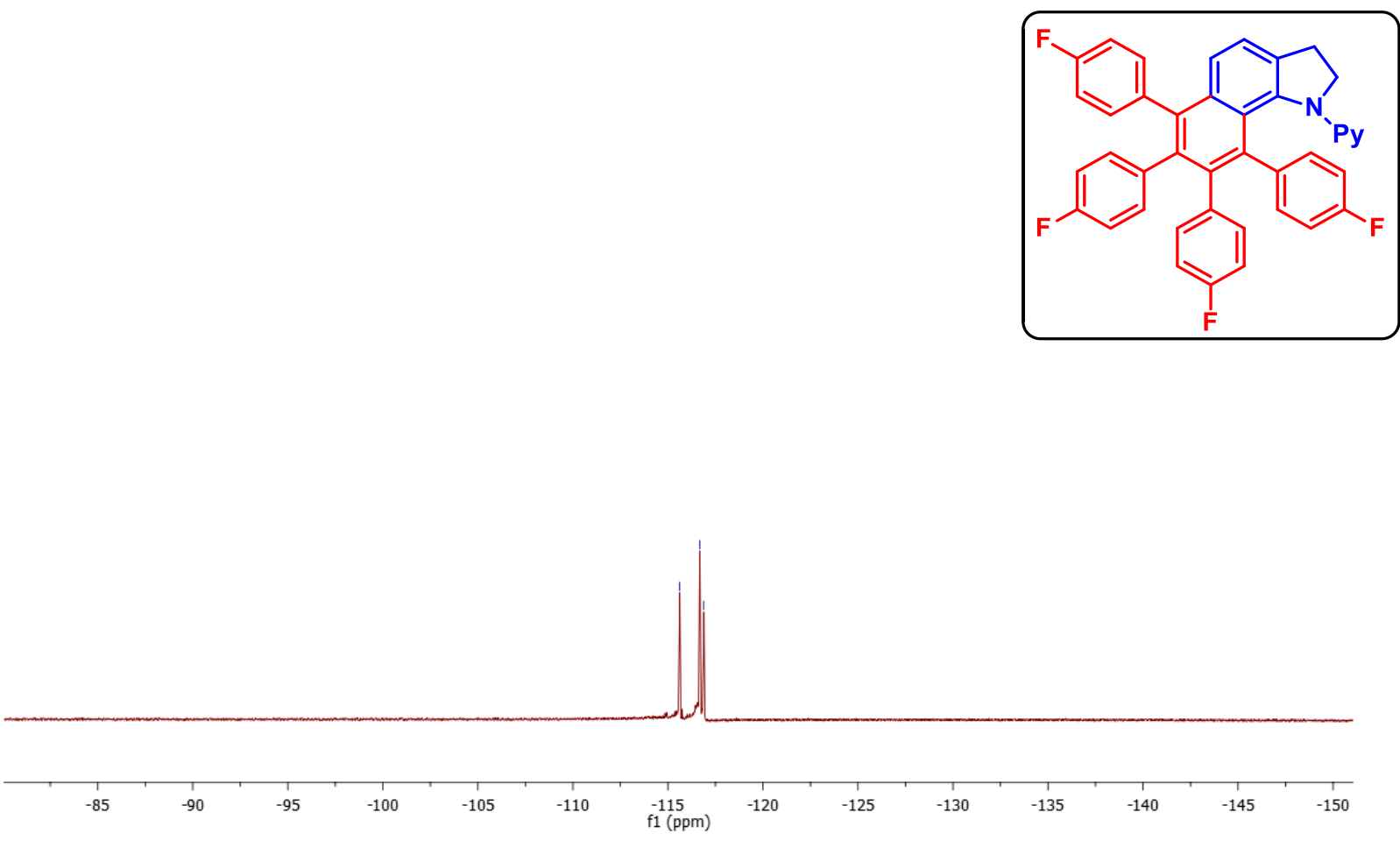


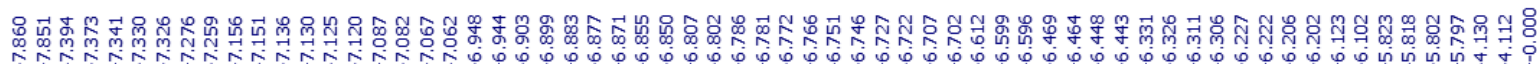
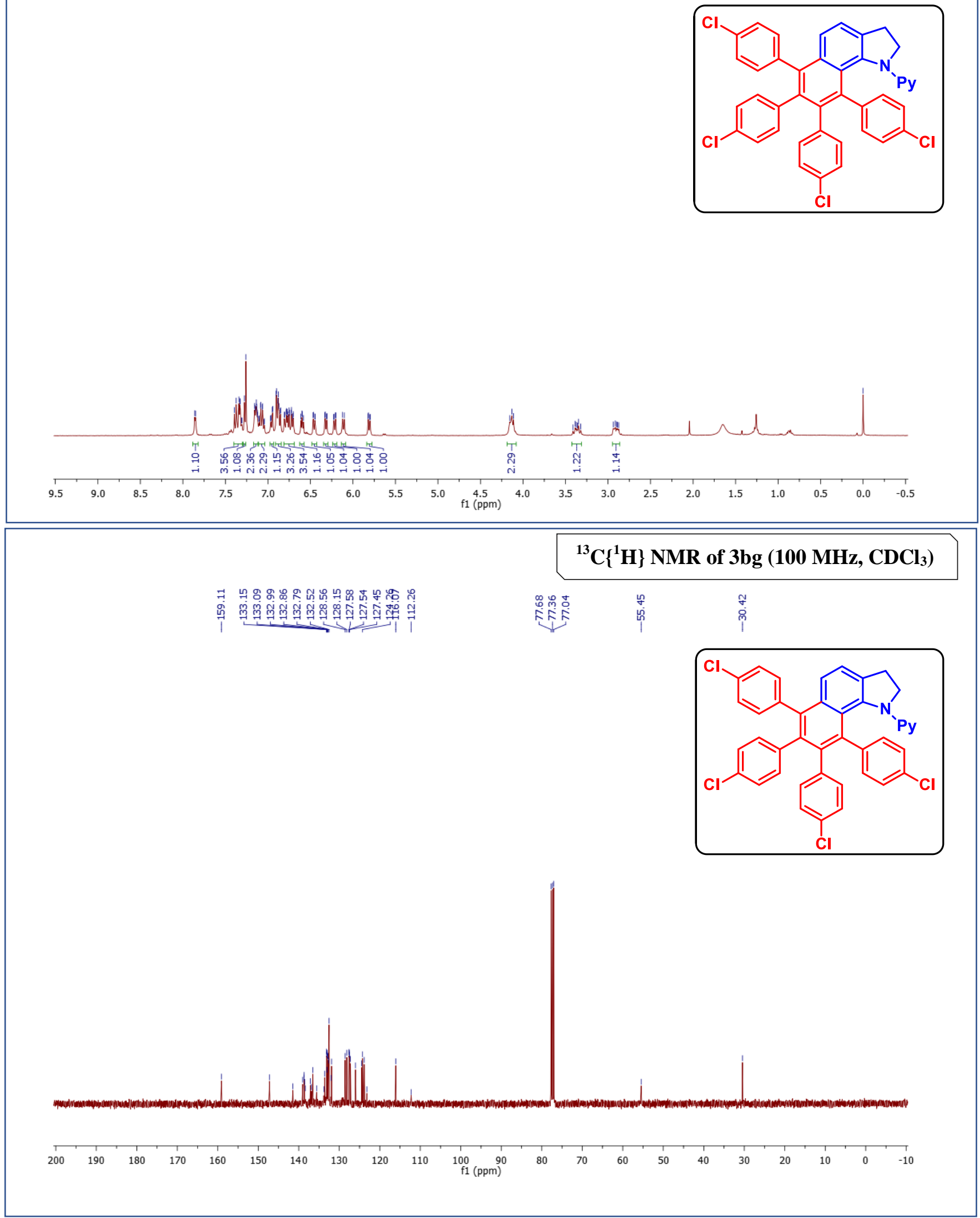


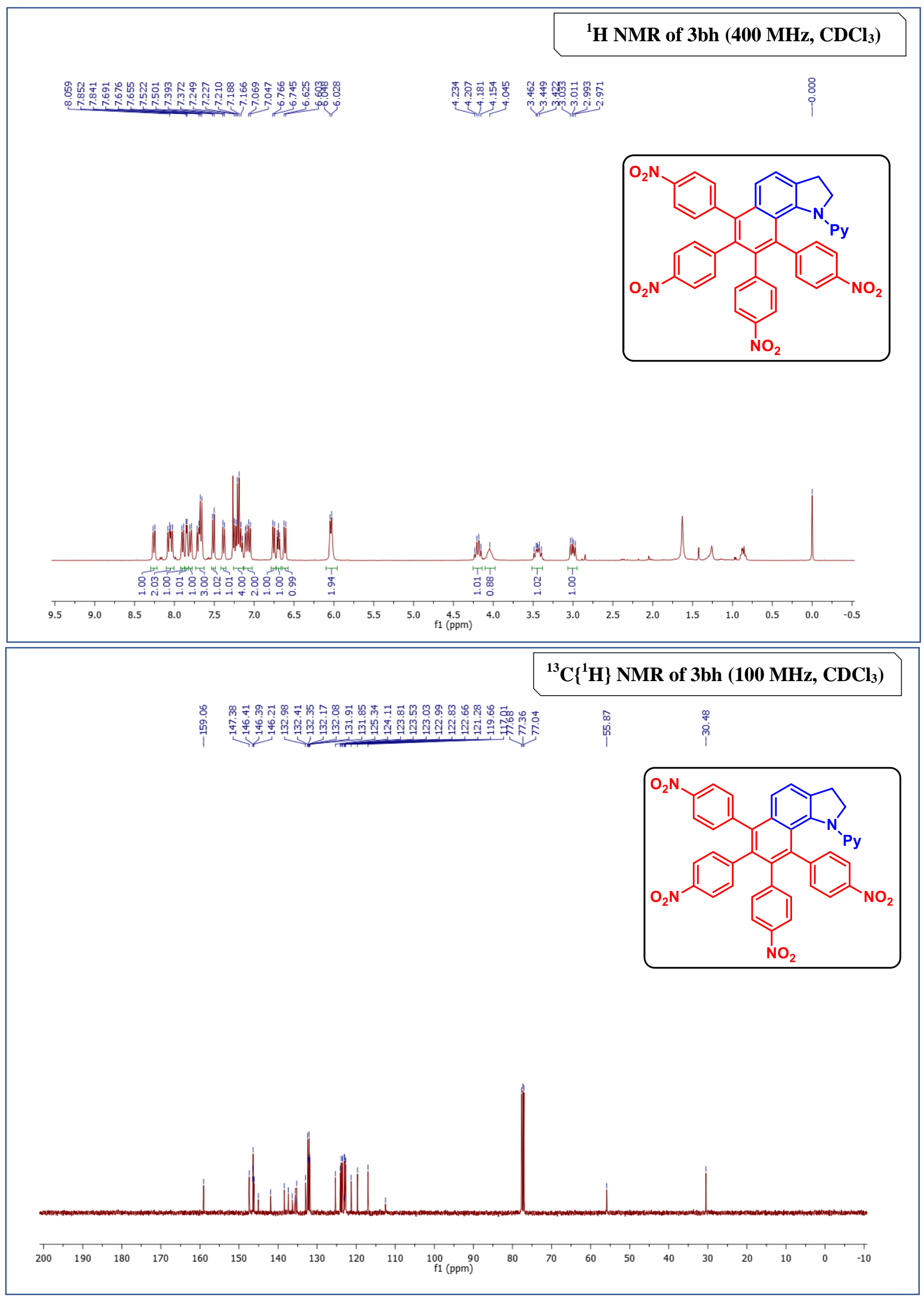




\section{${ }^{1} \mathrm{H}$ NMR of $5 \mathrm{aa}\left(400 \mathrm{MHz}, \mathrm{CDCl}_{3}\right)$}

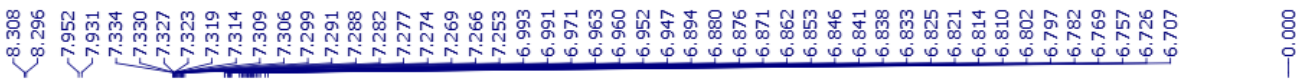
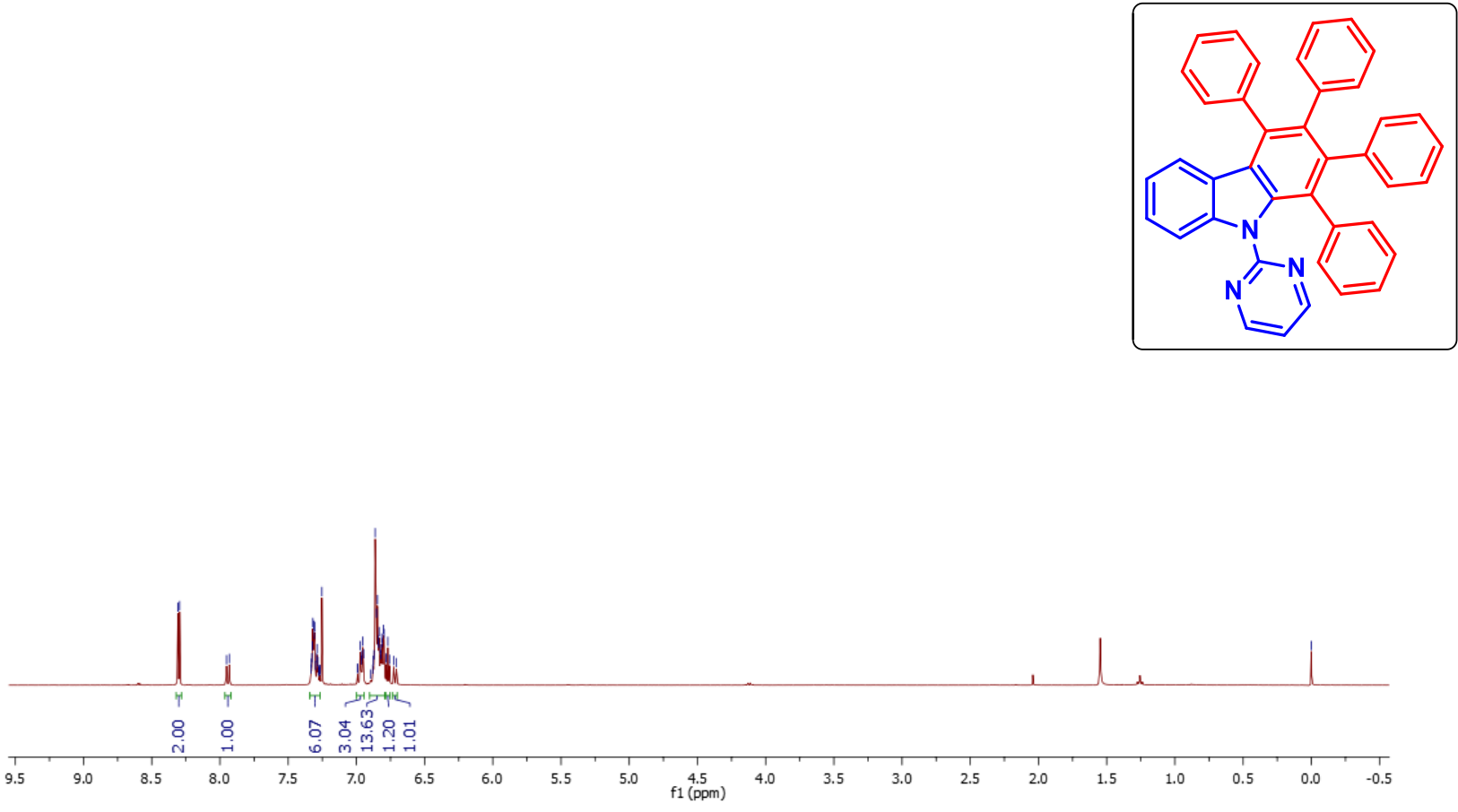

${ }^{13} \mathrm{C}\left\{{ }^{1} \mathrm{H}\right\}$ NMR of $5 \mathrm{aa}\left(100 \mathrm{MHz}, \mathrm{CDCl}_{3}\right)$

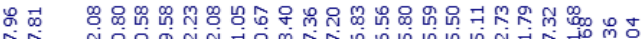

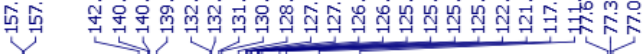
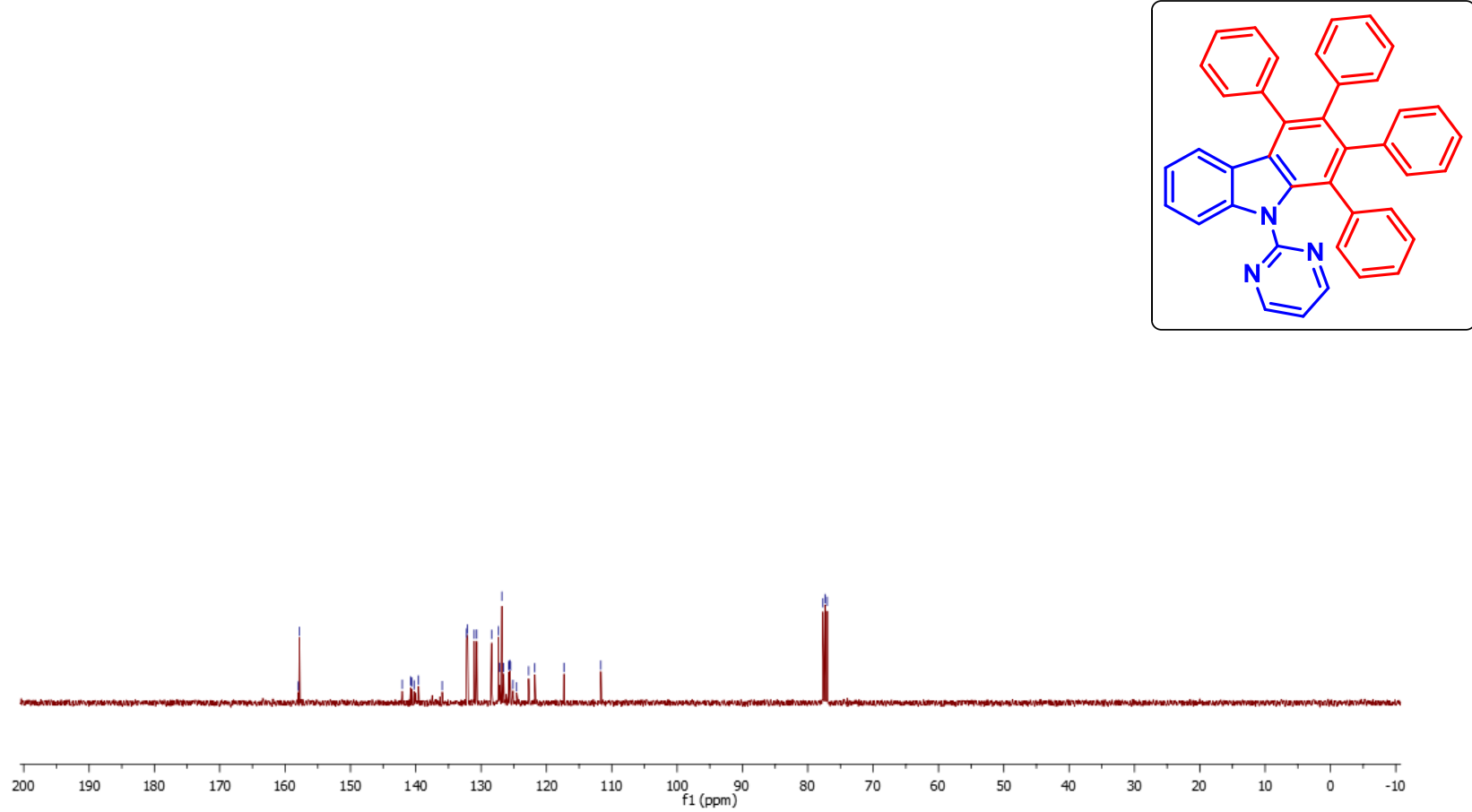


\section{${ }^{1} \mathrm{H}$ NMR of $5 \mathrm{ba}\left(400 \mathrm{MHz}, \mathrm{CDCl}_{3}\right)$}

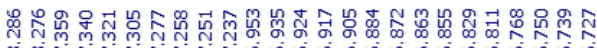

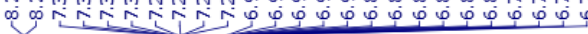

o
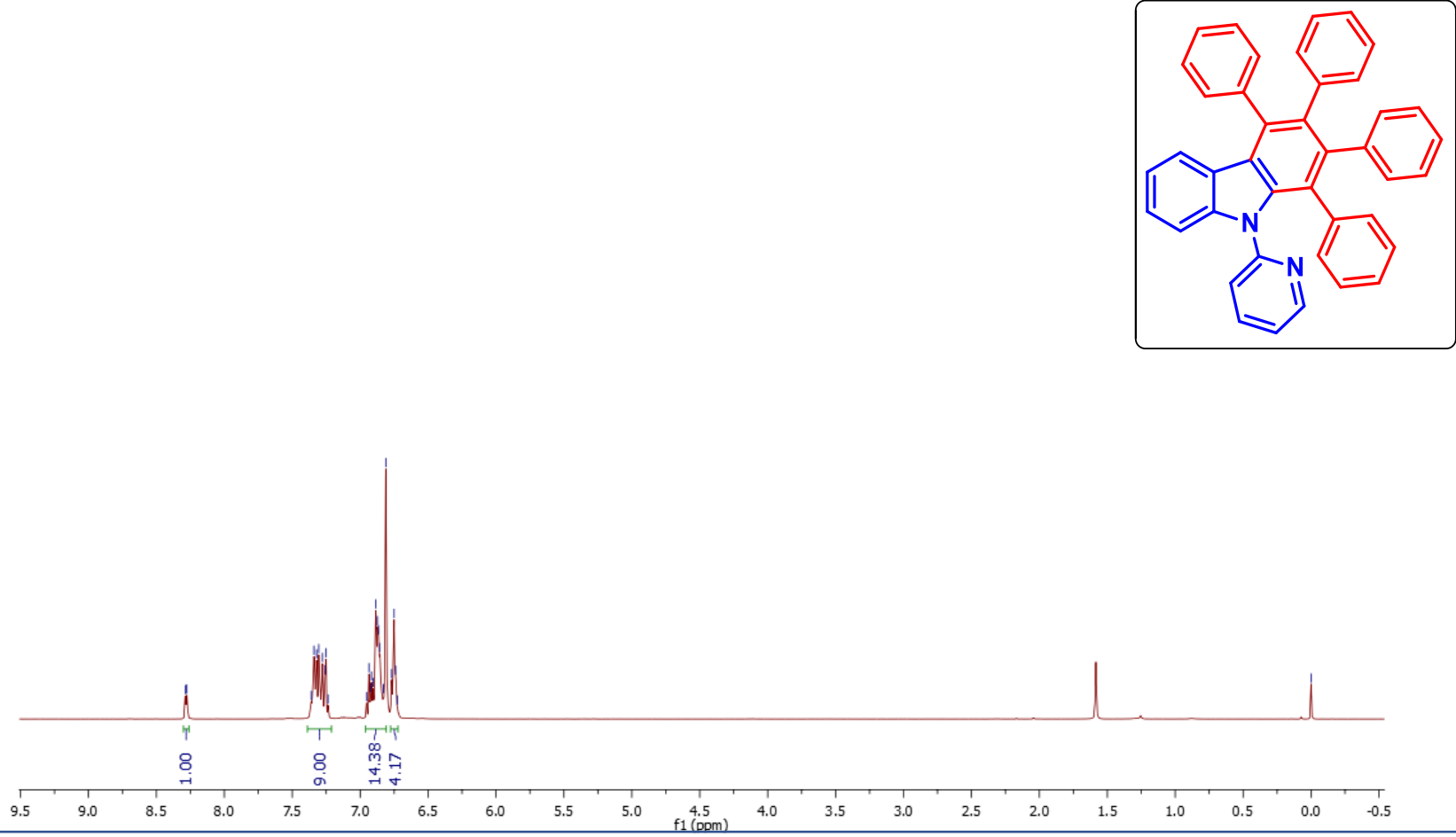

${ }^{13} \mathrm{C}\left\{{ }^{1} \mathrm{H}\right\}$ NMR of $5 \mathrm{ba}\left(100 \mathrm{MHz}, \mathrm{CDCl}_{3}\right)$

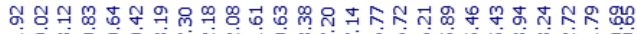

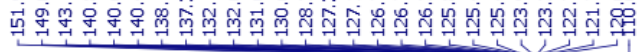

究篎
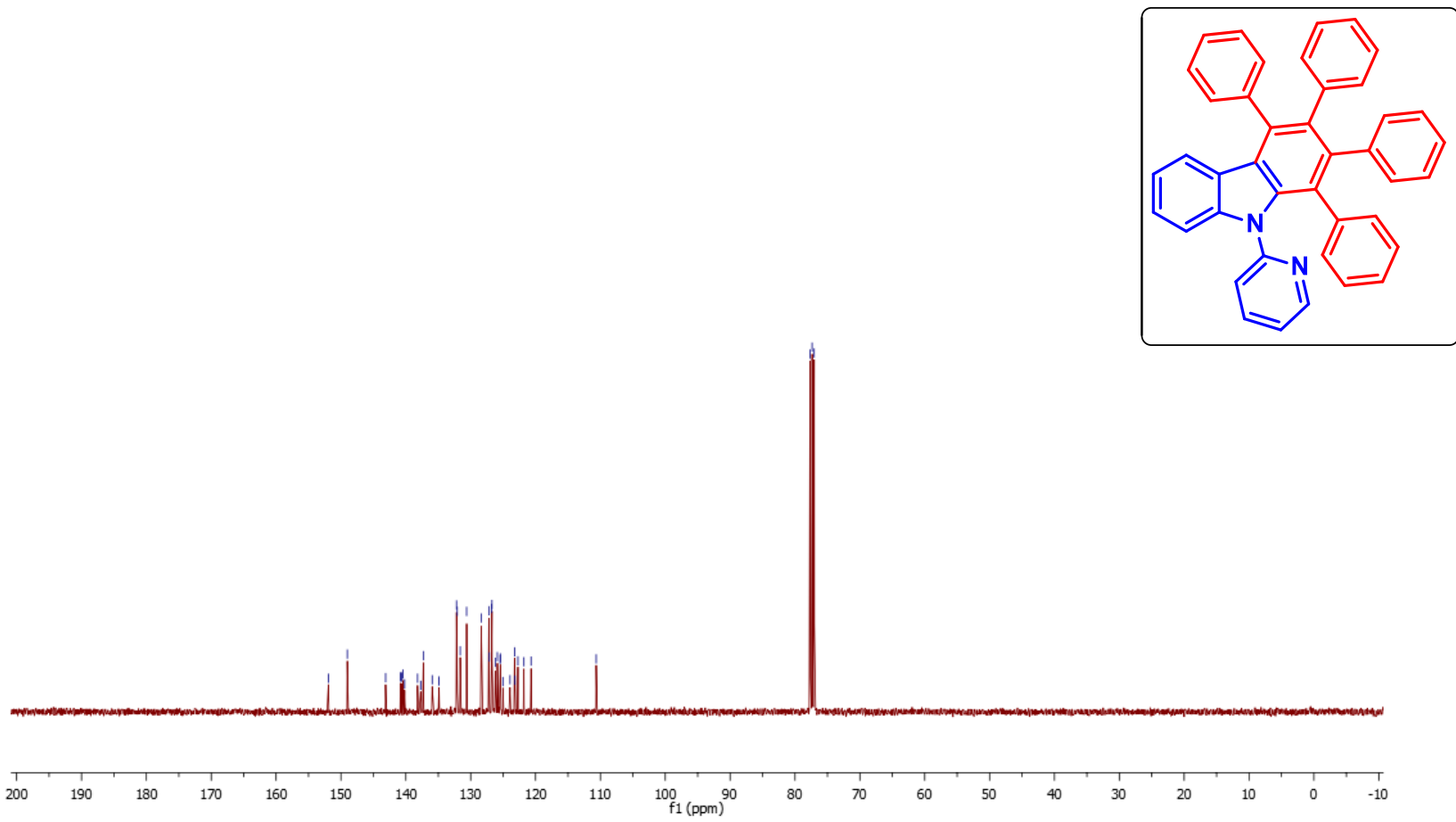
${ }^{1} \mathrm{H}$ NMR of $5 \mathrm{ca}\left(400 \mathrm{MHz}, \mathrm{CDCl}_{3}\right)$

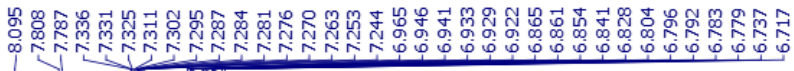

$\underset{\substack{1 \\ 1}}{\stackrel{\pi}{1}}$

:
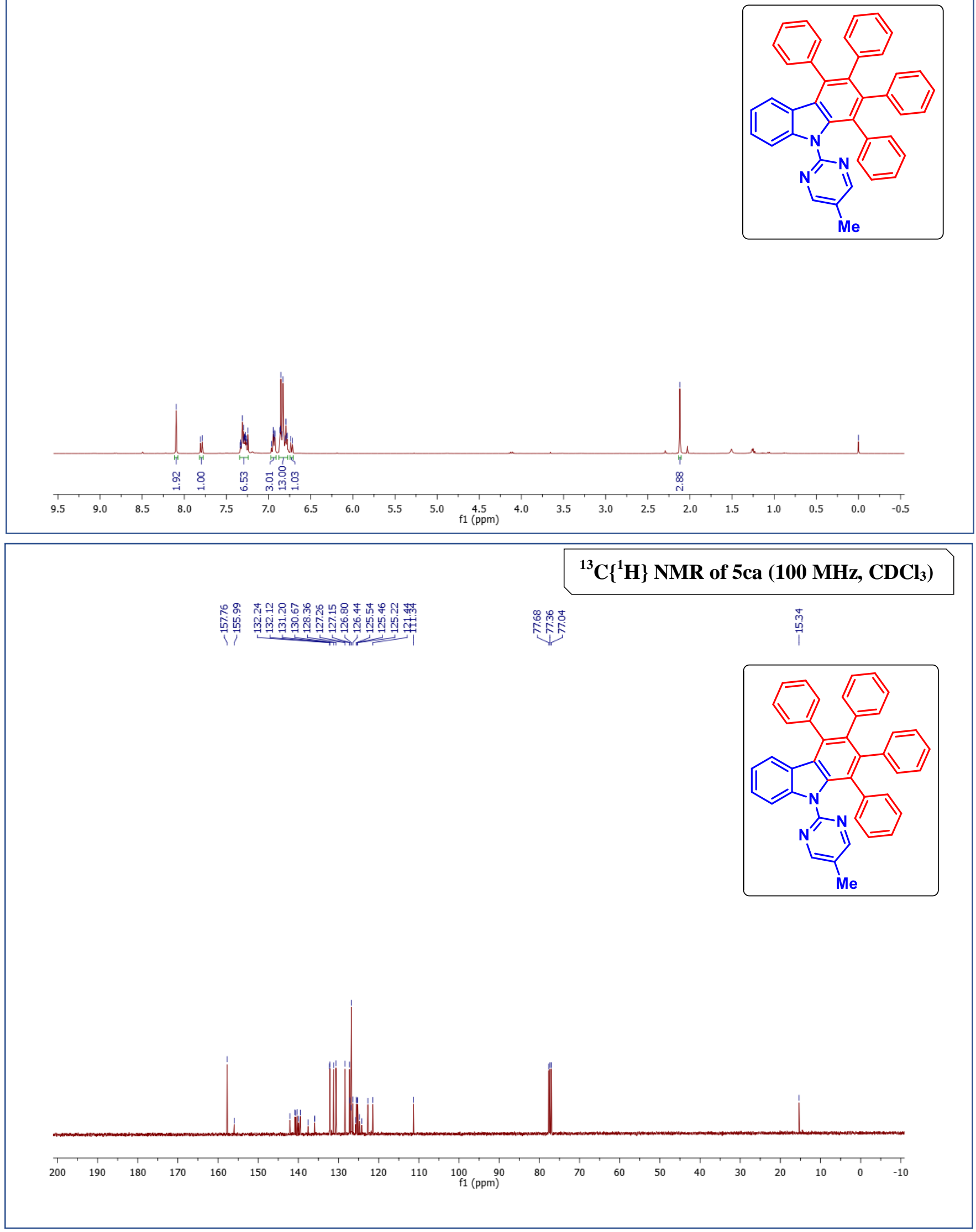


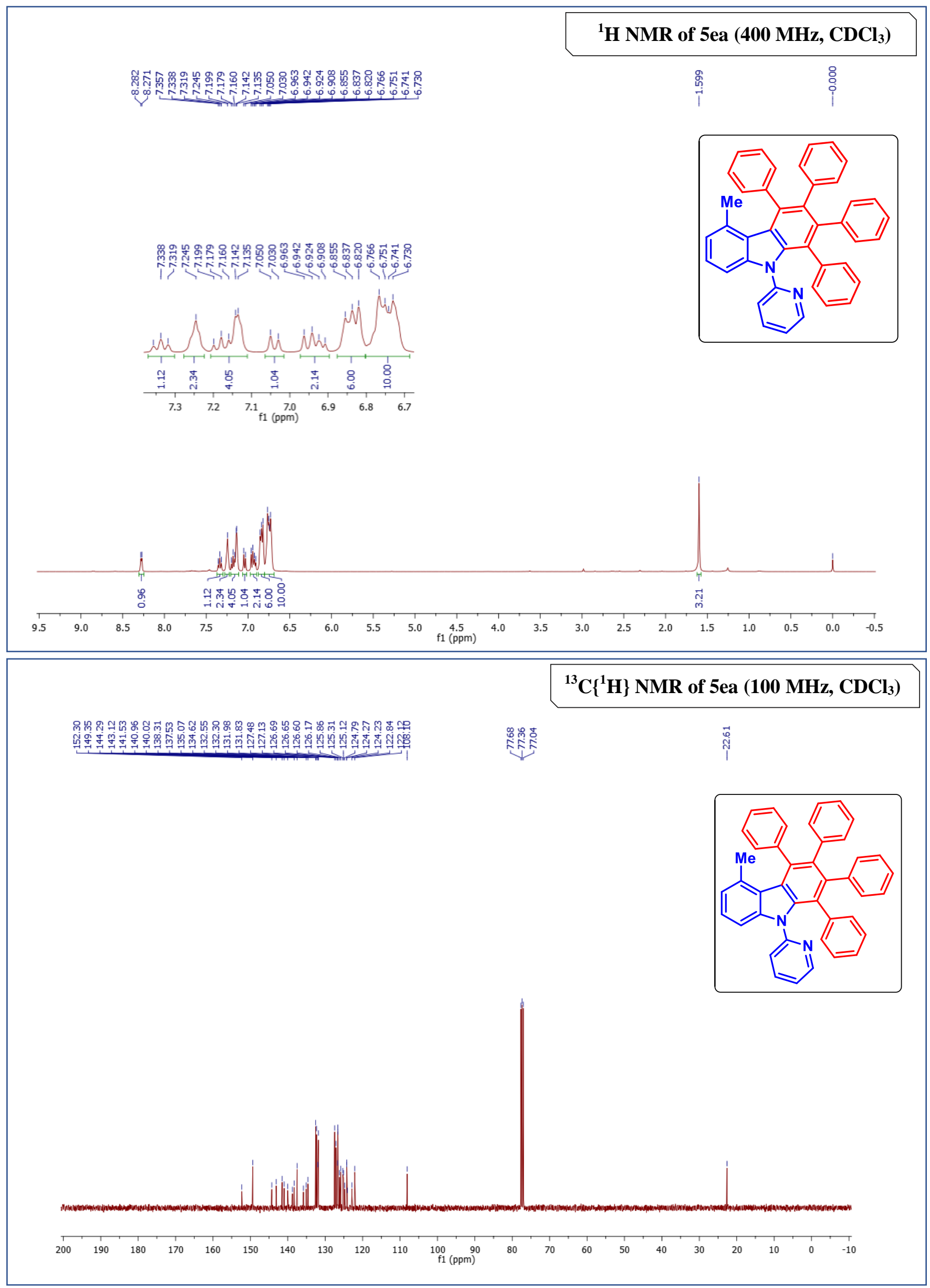




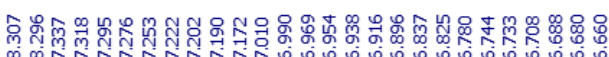
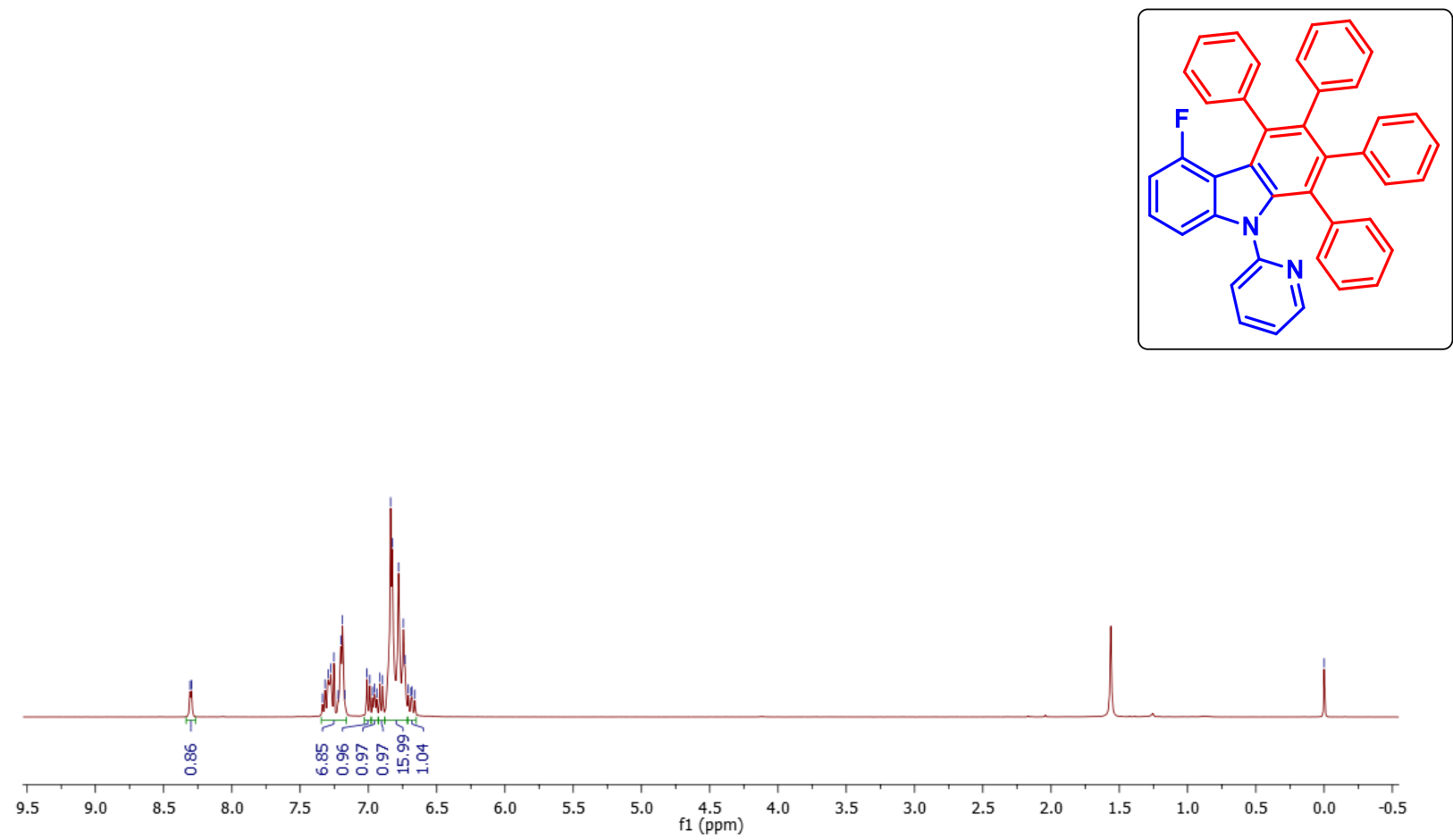

${ }^{13} \mathrm{C}\left\{{ }^{1} \mathrm{H}\right\}$ NMR of $5 f a\left(176 \mathrm{MHz}, \mathrm{CDCl}_{3}\right)$

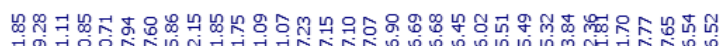

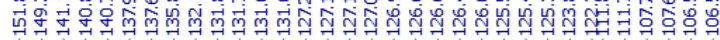
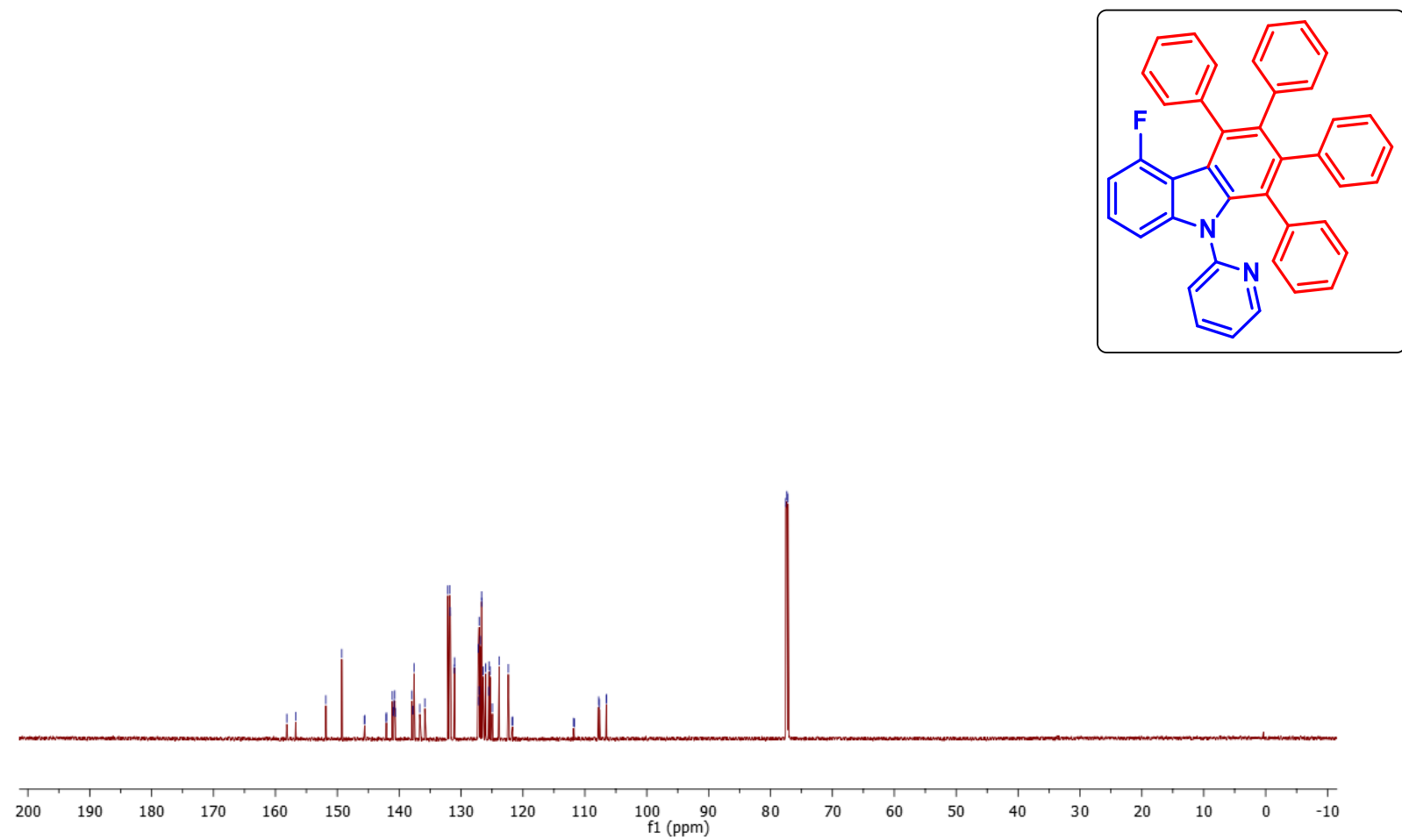


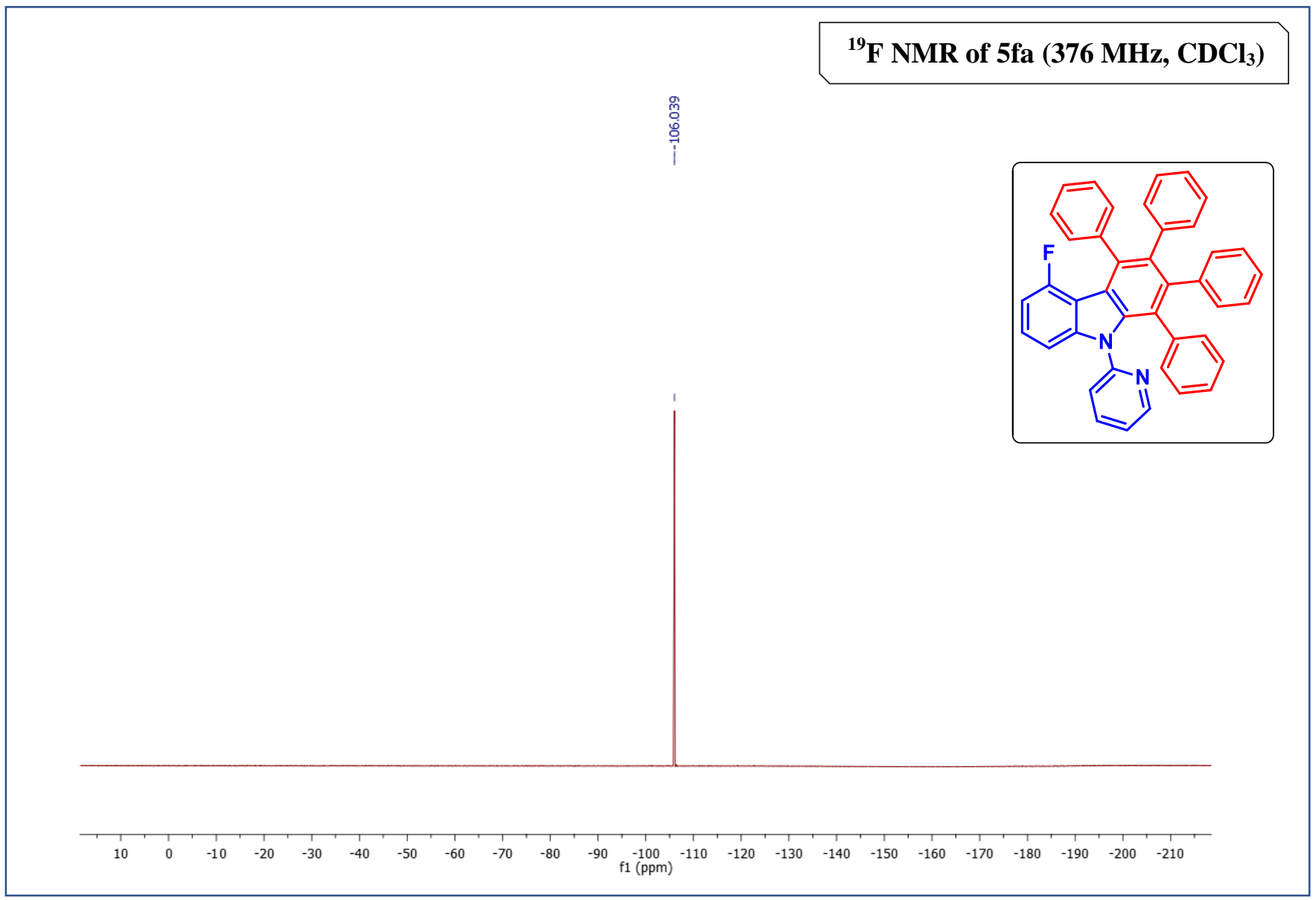



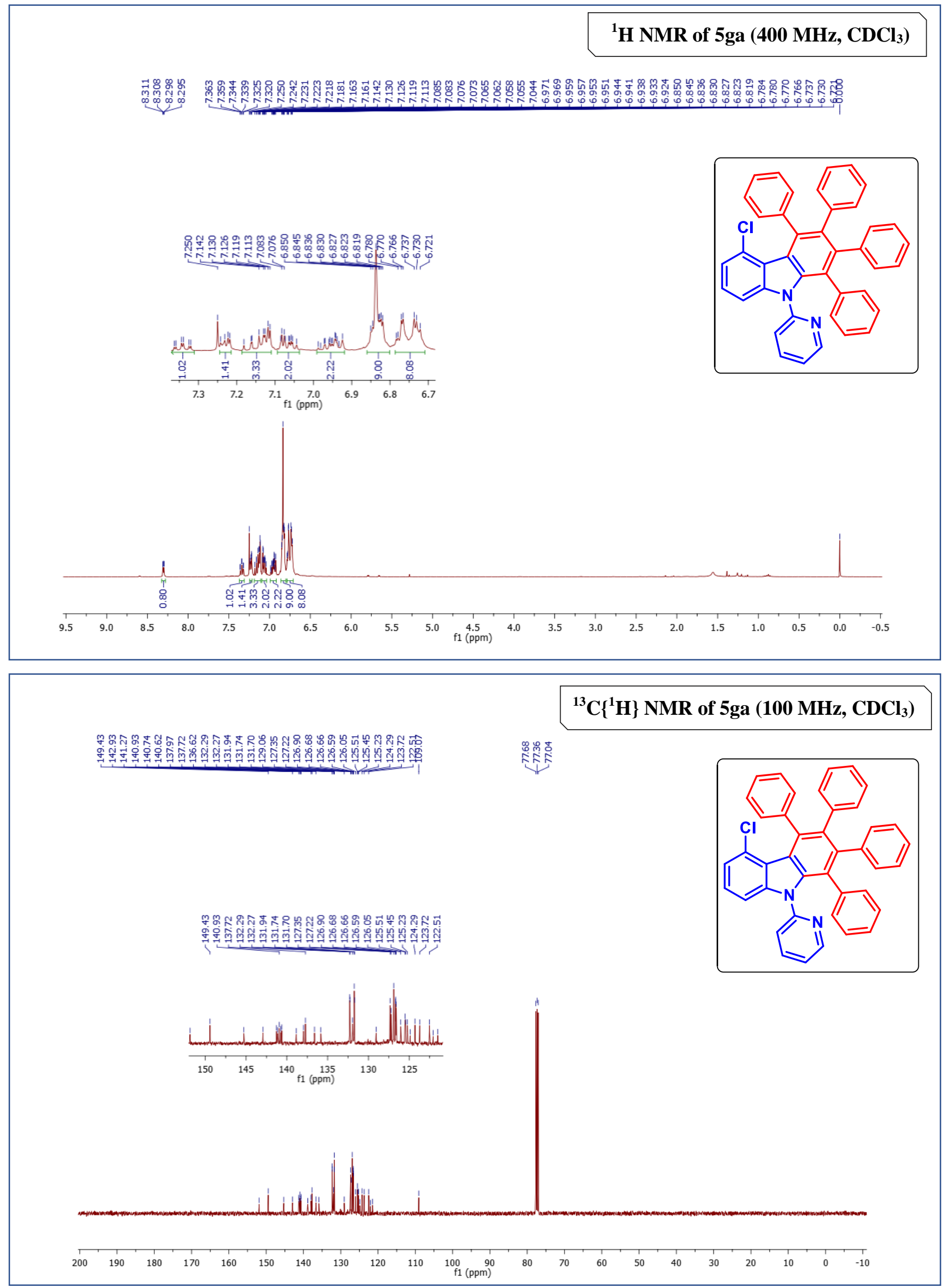

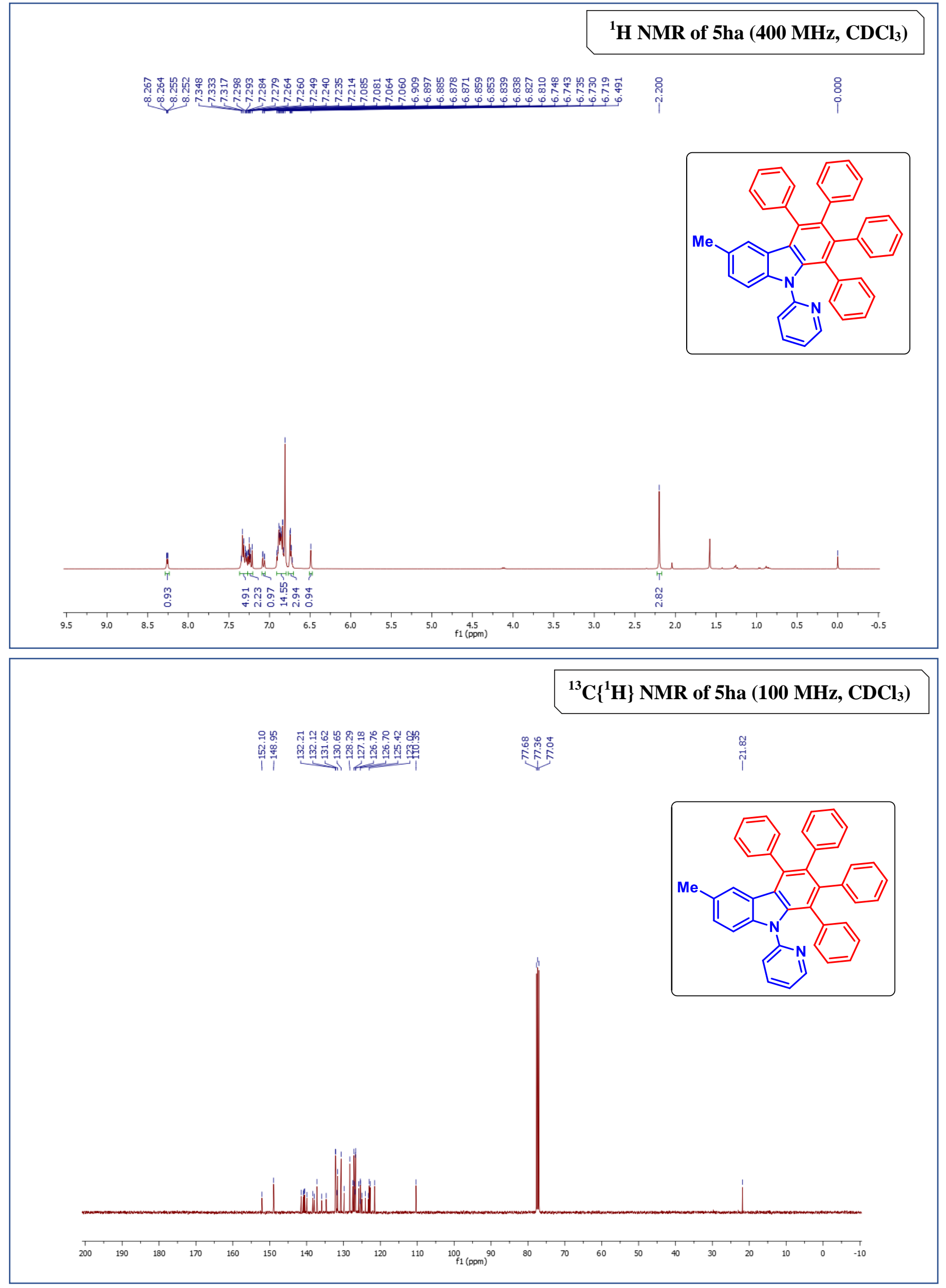


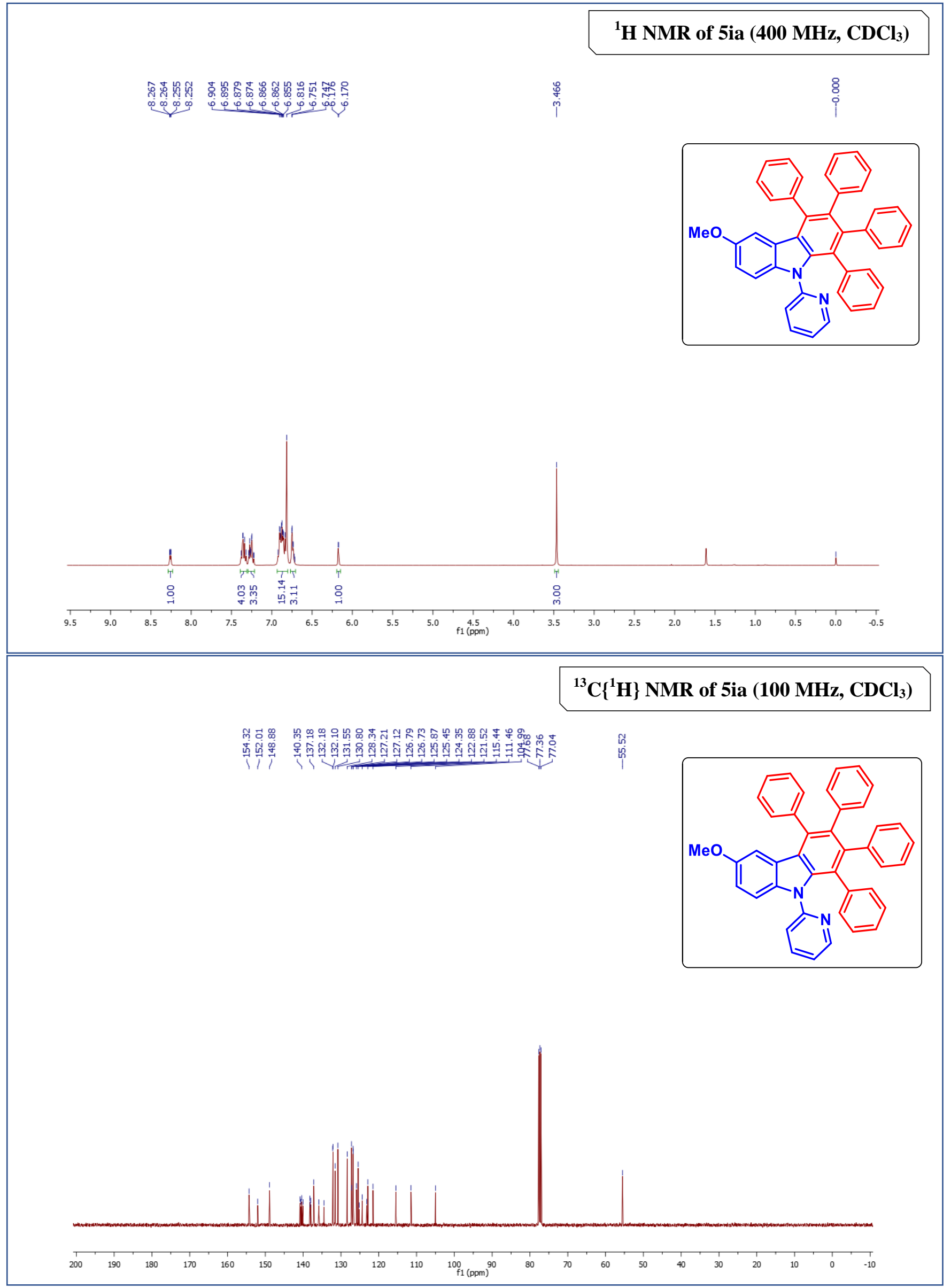



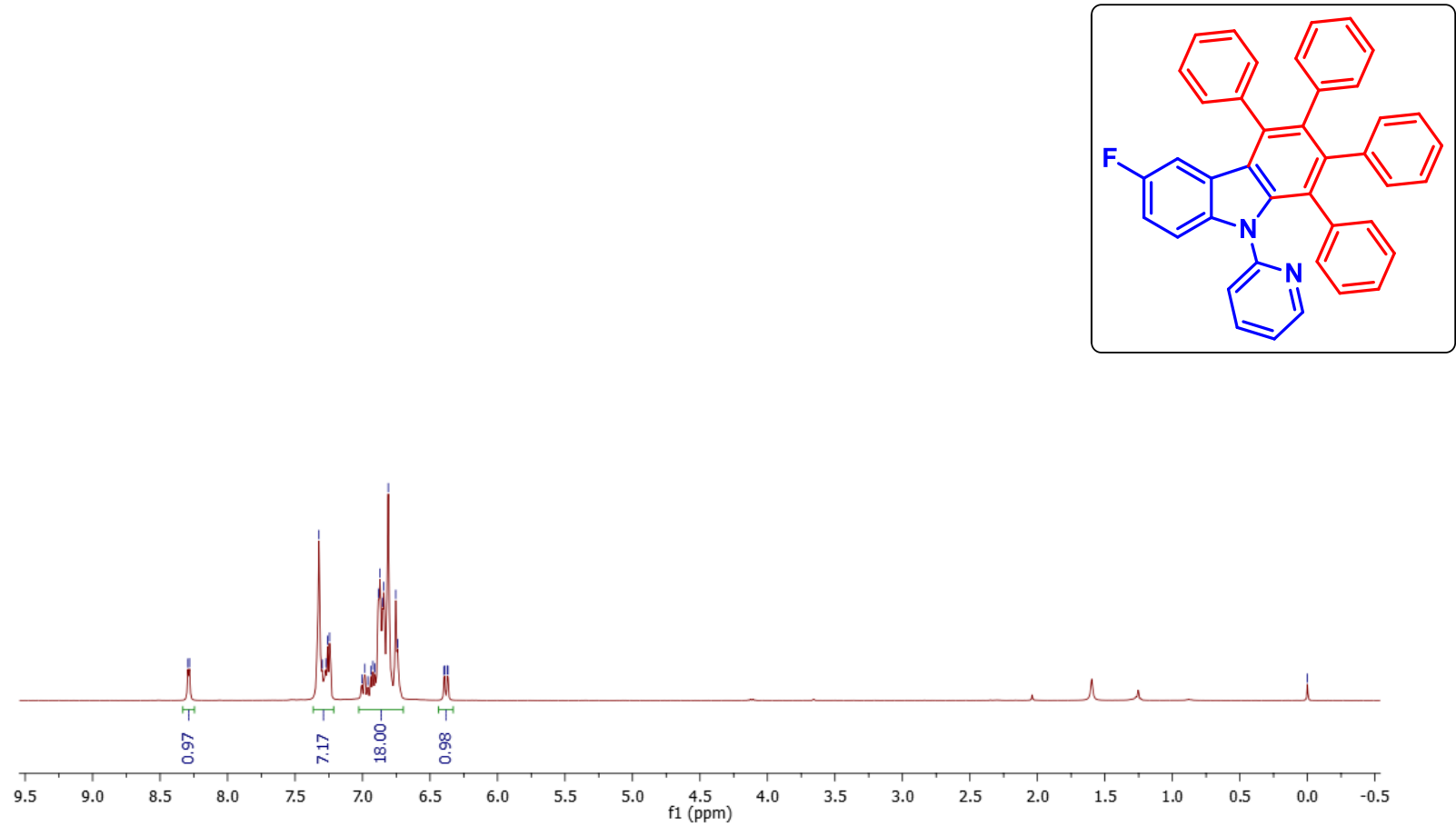

${ }^{13} \mathrm{C}\left\{{ }^{1} \mathrm{H}\right\}$ NMR of $5 \mathrm{ja}\left(100 \mathrm{MHz}, \mathrm{CDCl}_{3}\right)$

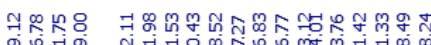

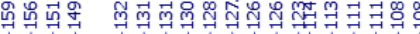

11 रो 는
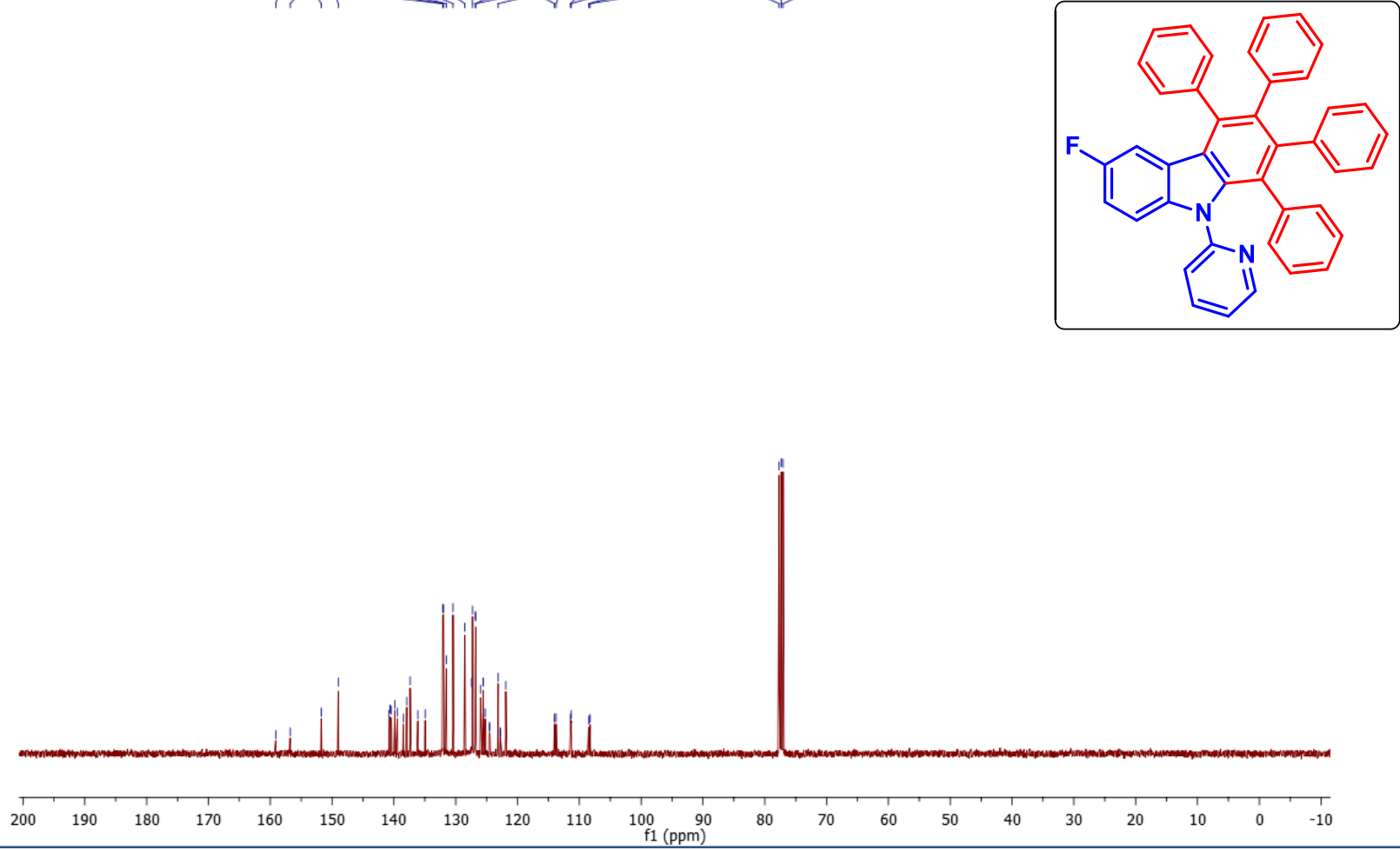
${ }^{19}$ F NMR of 5 ja $\left(376 \mathrm{MHz}, \mathrm{CDCl}_{3}\right)$

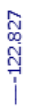
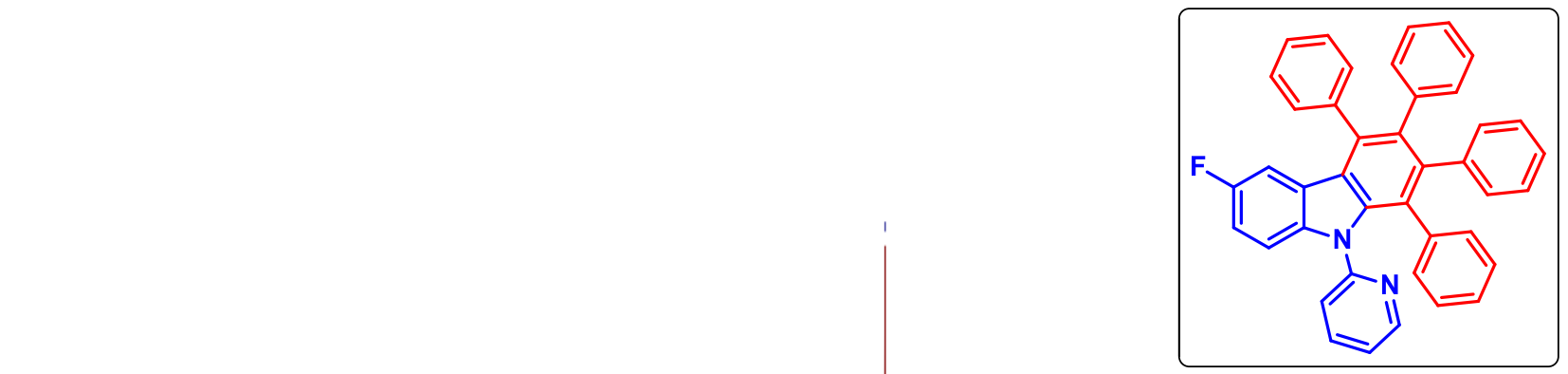

$\begin{array}{llll}10 & 0 & -10 & -20\end{array}$ 

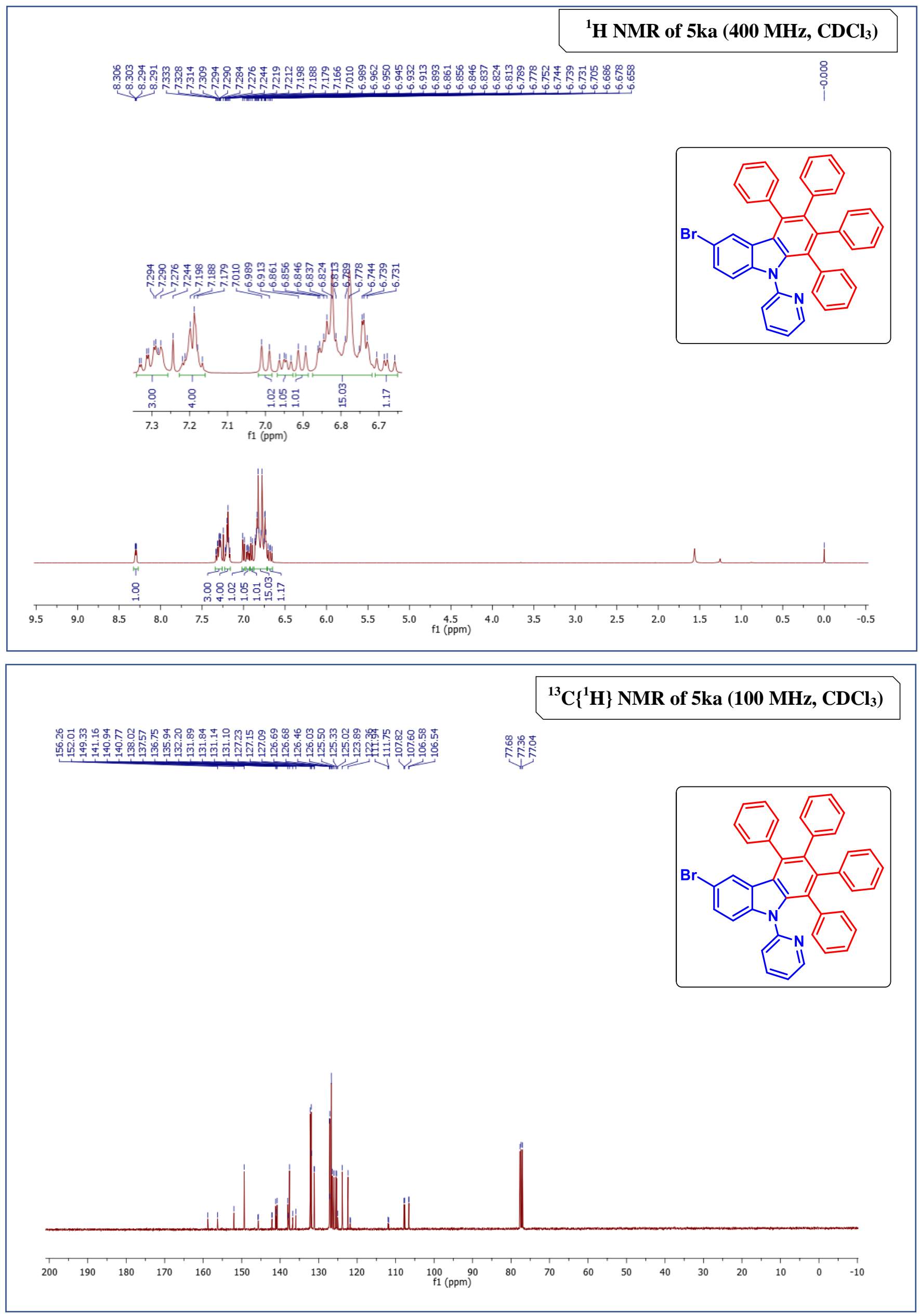

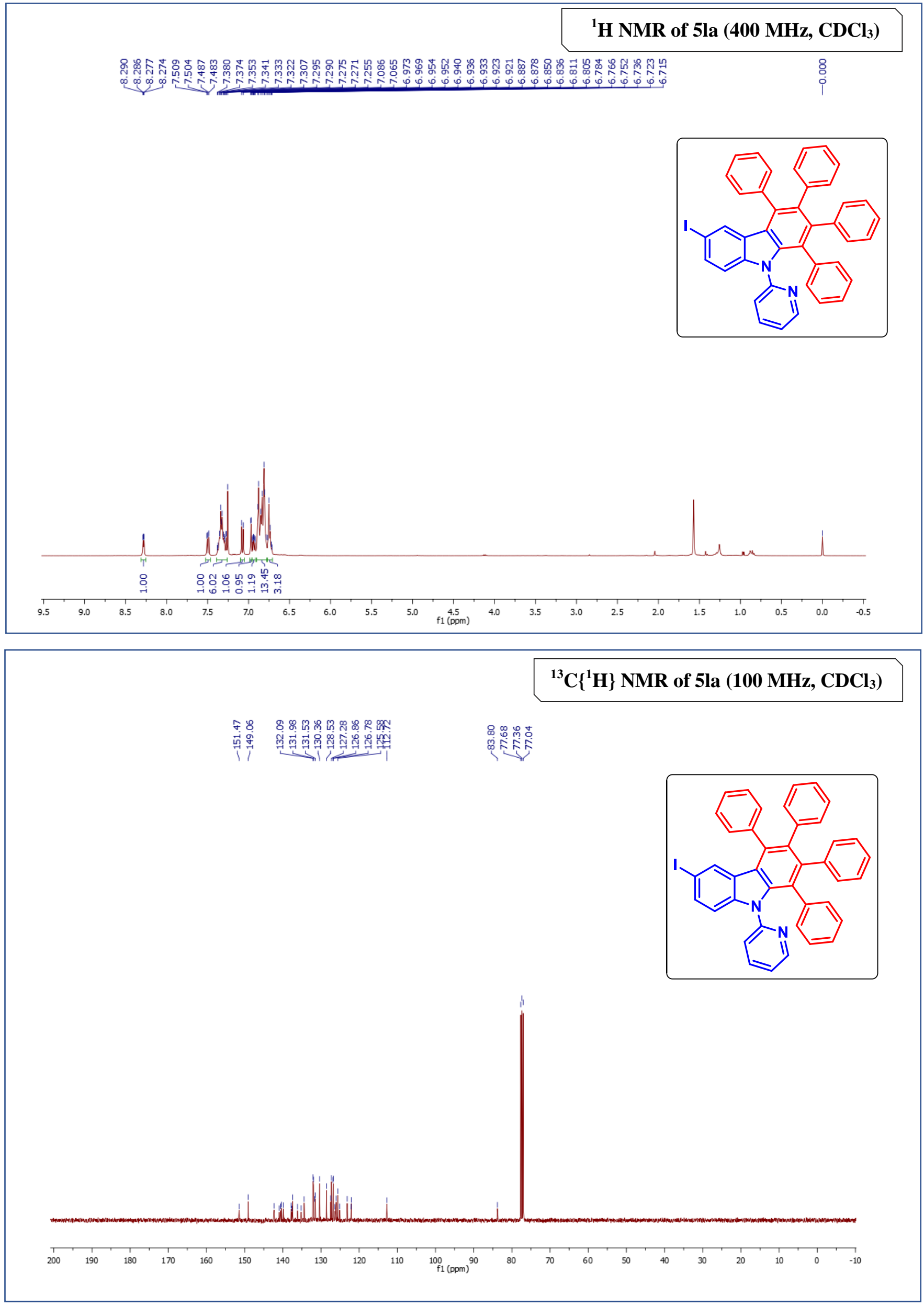

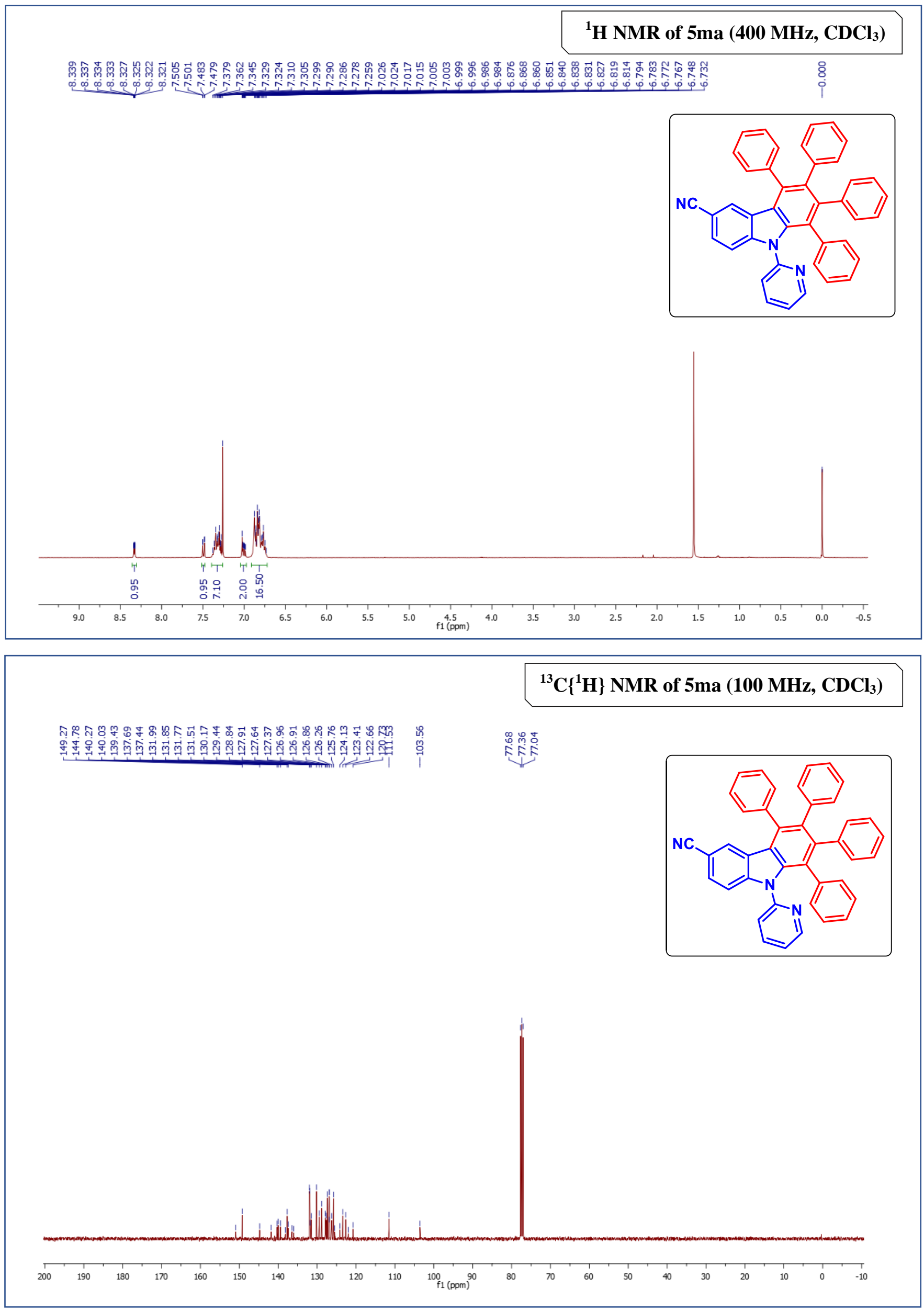

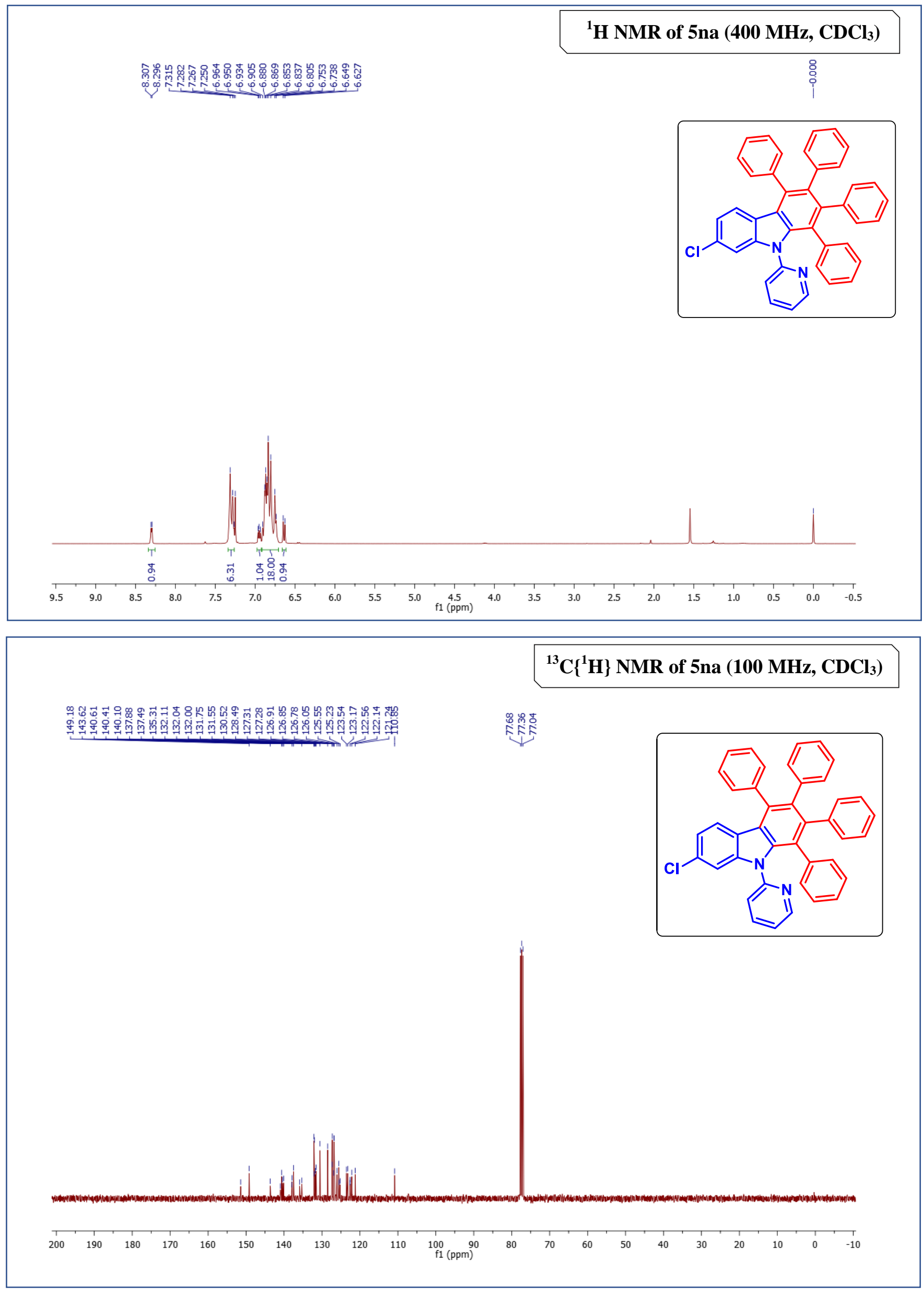


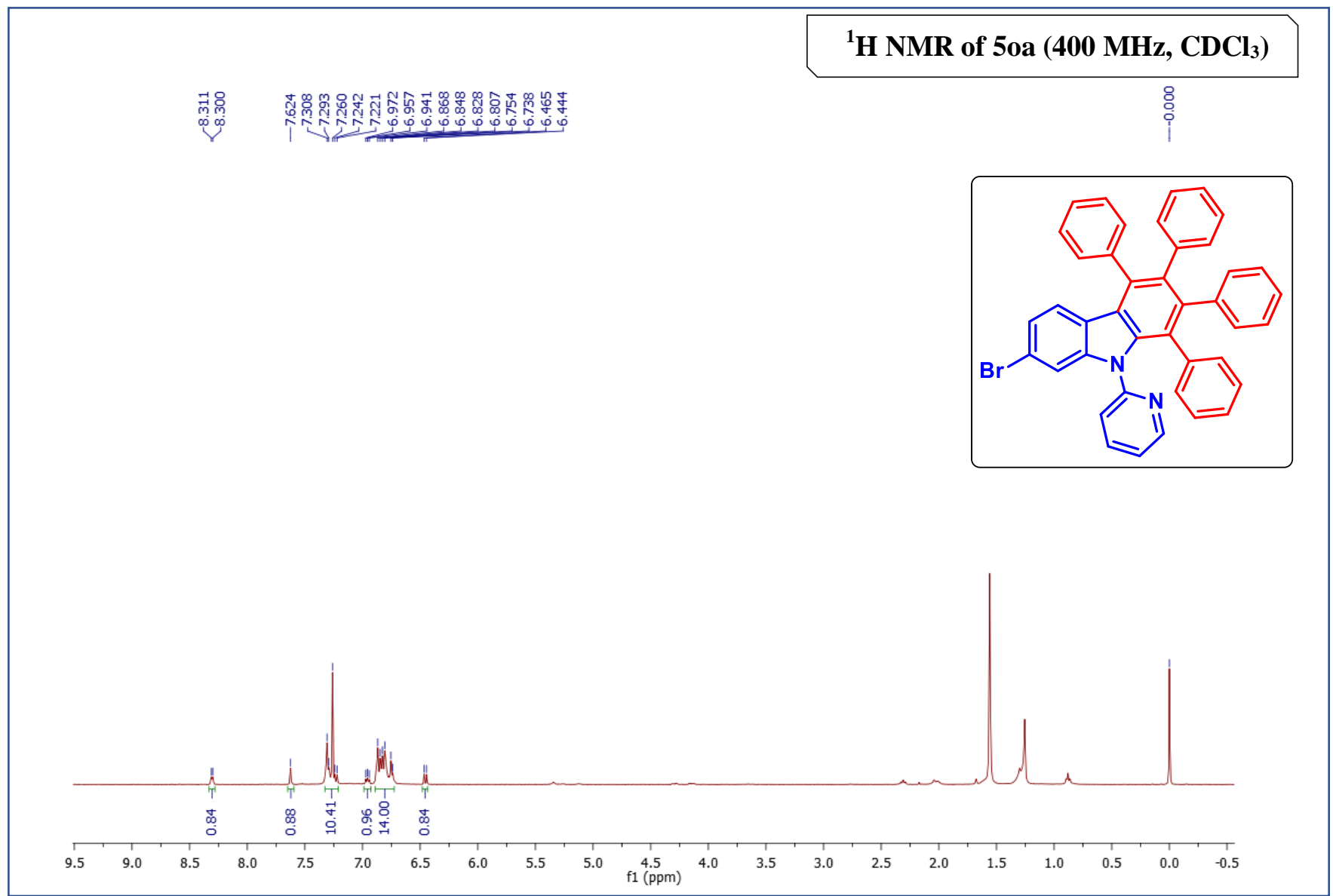

${ }^{13} \mathrm{C}\left\{{ }^{1} \mathrm{H}\right\}$ NMR of $50 a\left(100 \mathrm{MHz}, \mathrm{CDCl}_{3}\right)$

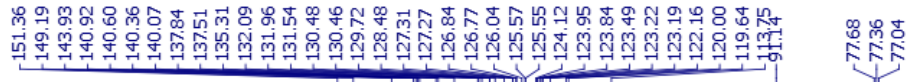
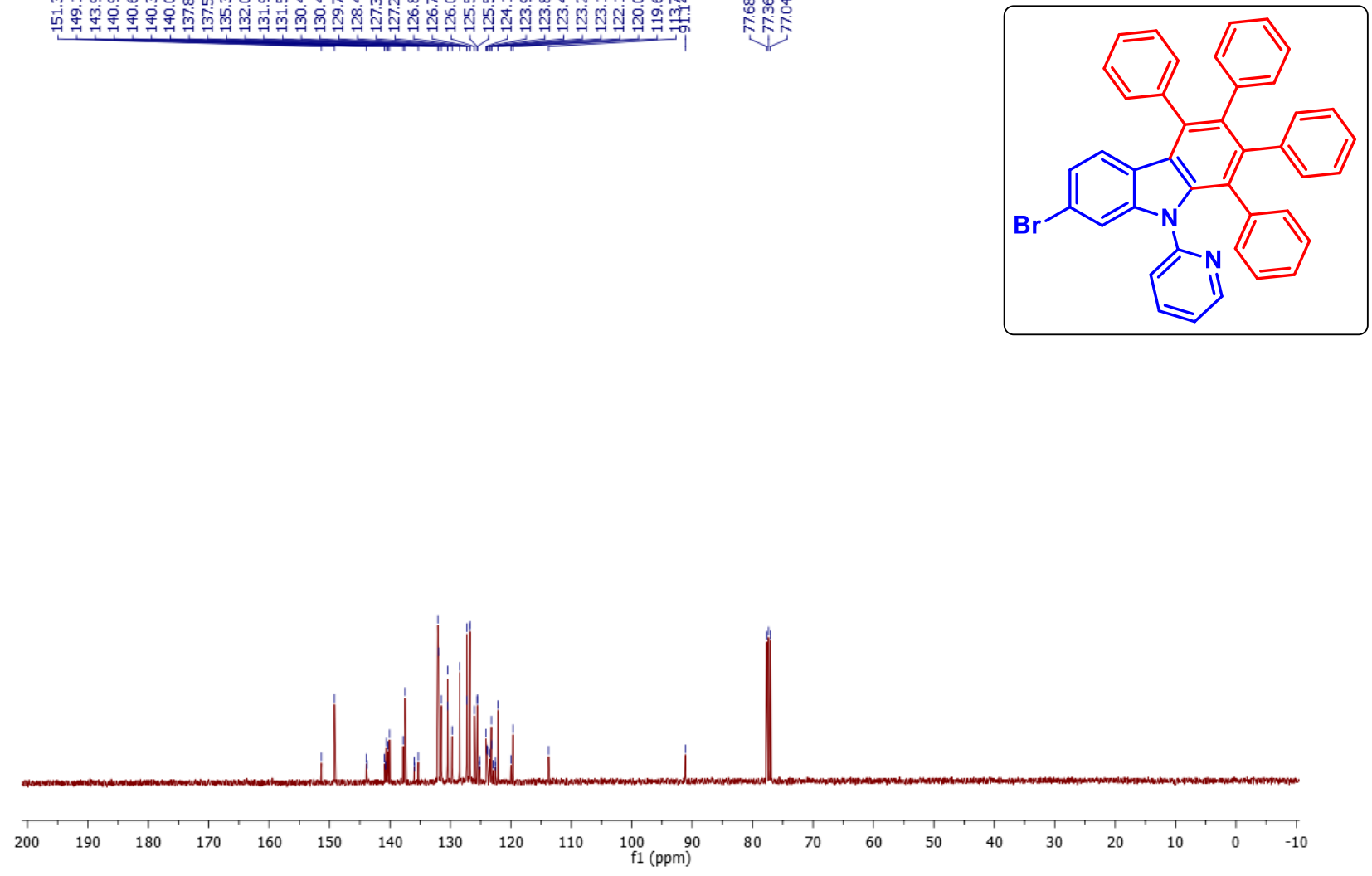


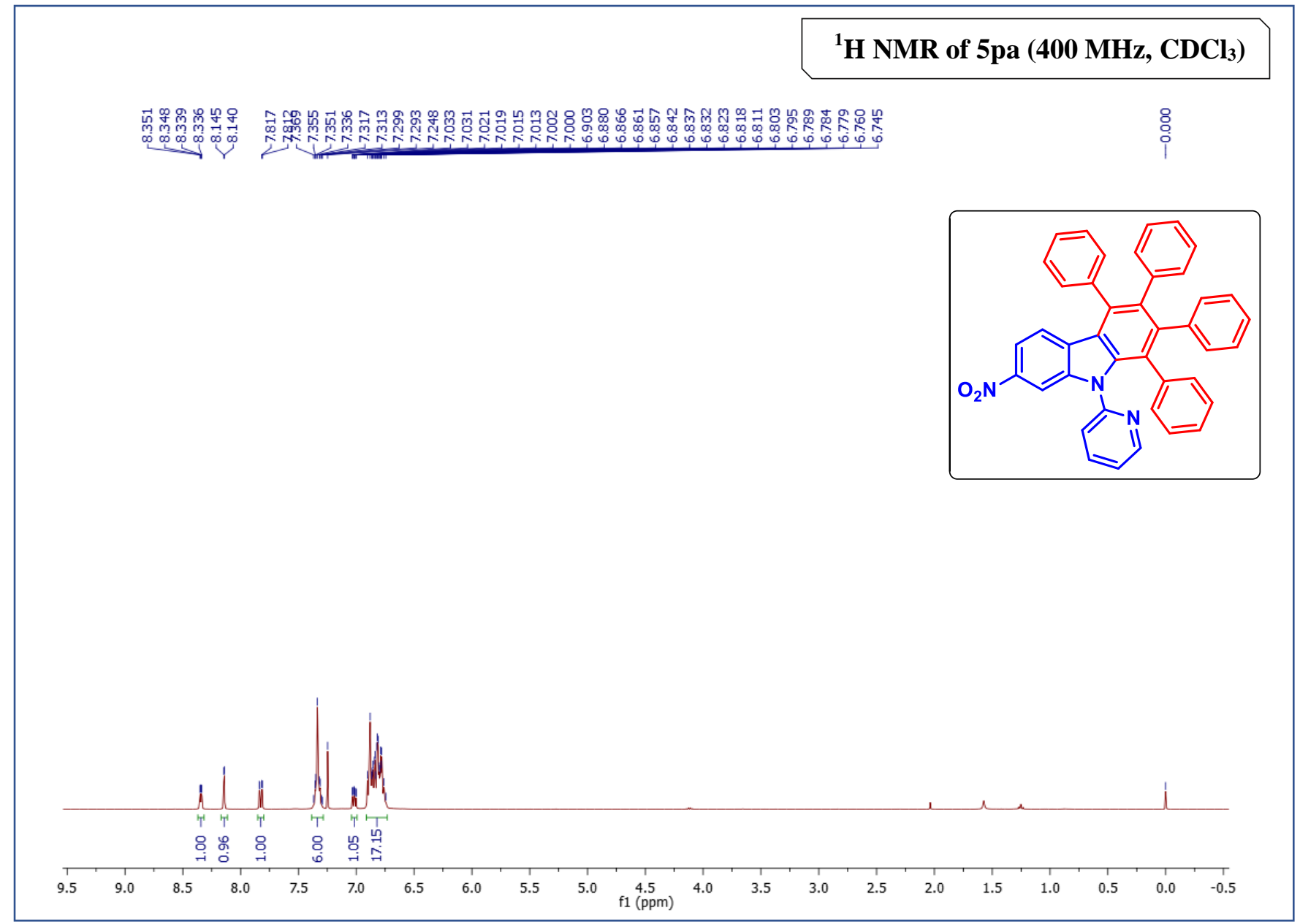

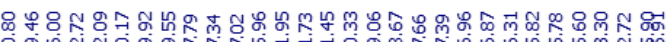

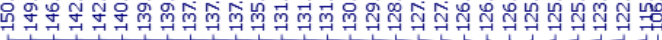

${ }^{13} \mathrm{C}\left\{{ }^{1} \mathrm{H}\right\}$ NMR of 5 pa $\left(100 \mathrm{MHz}, \mathrm{CDCl}_{3}\right)$
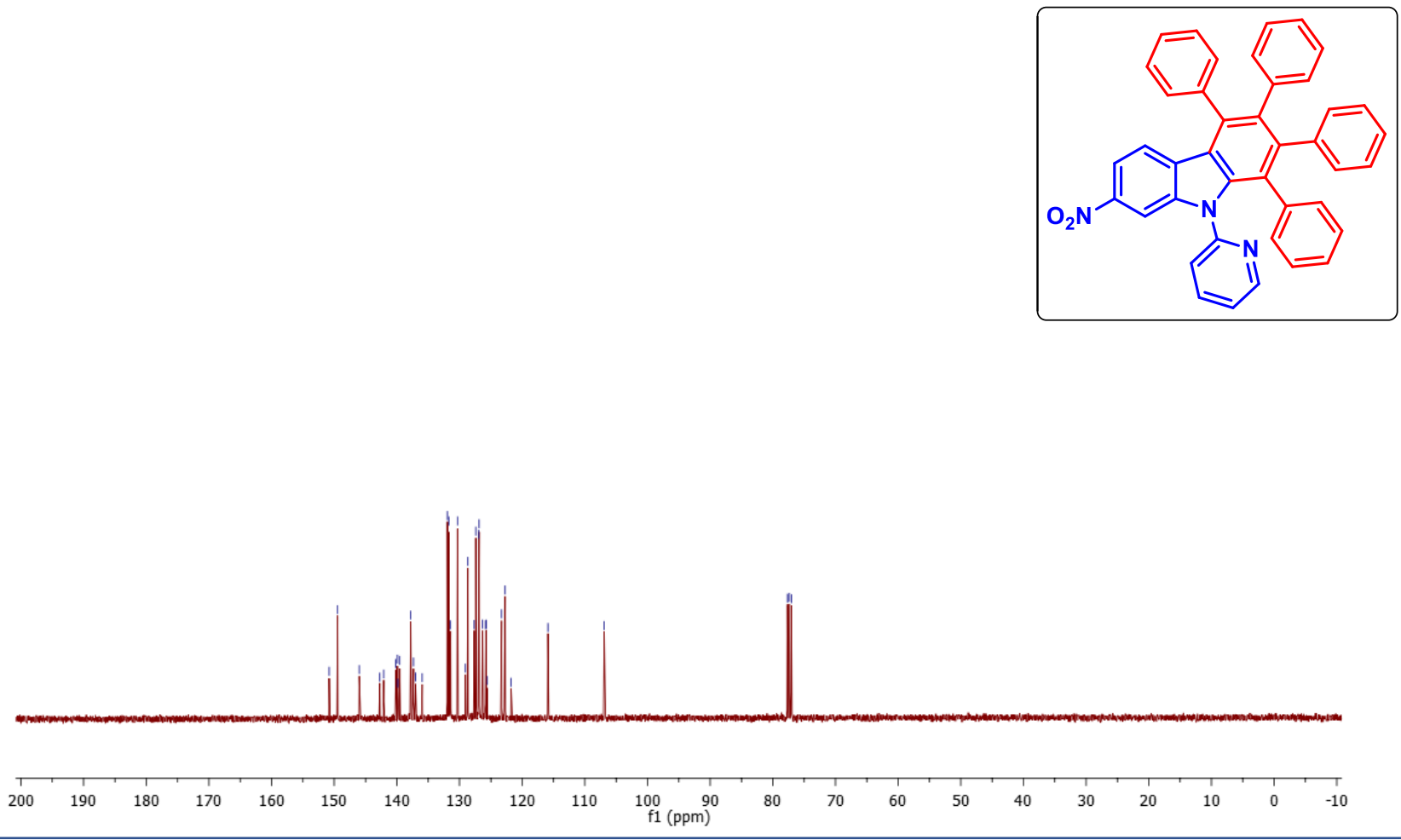


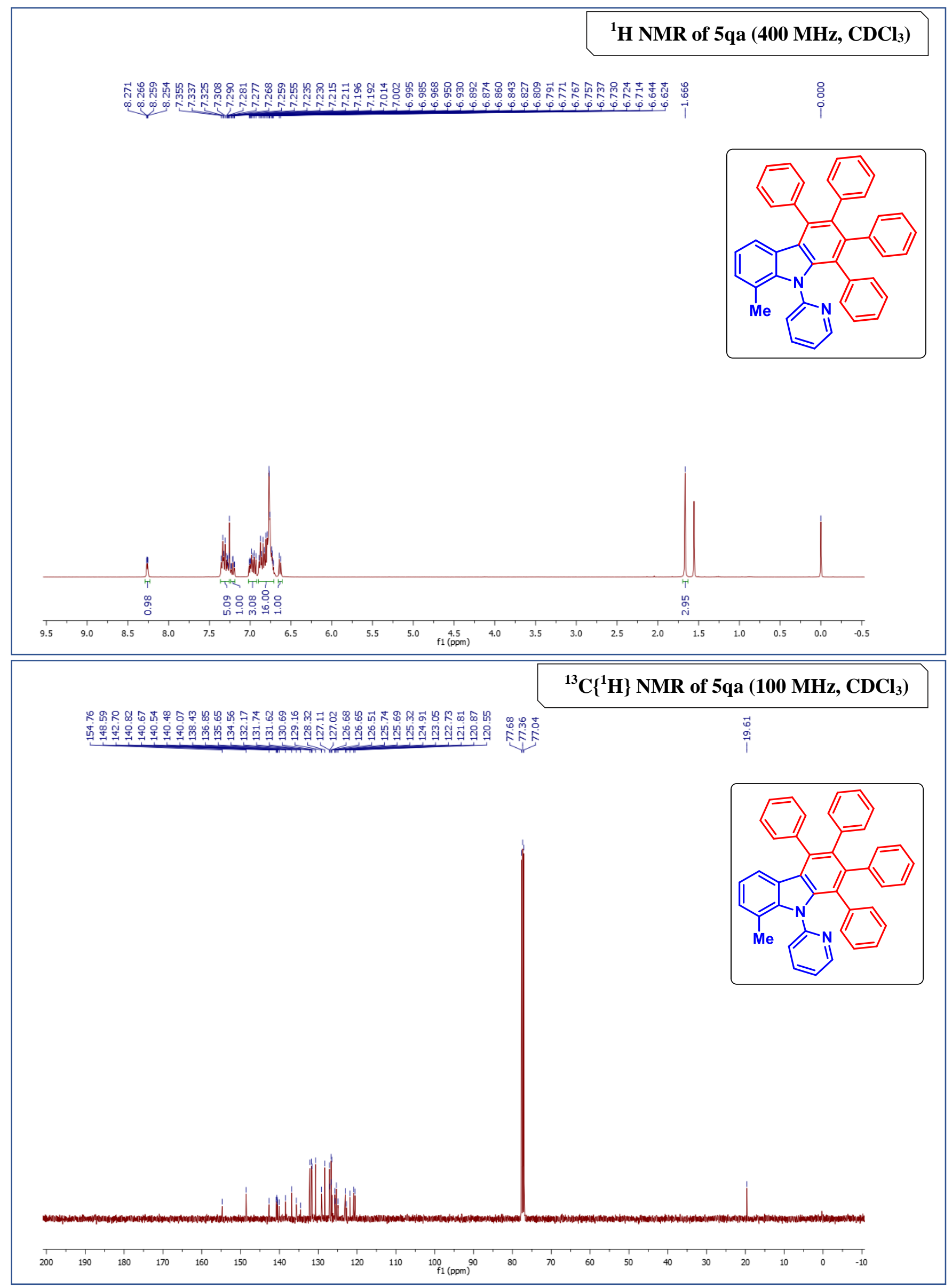




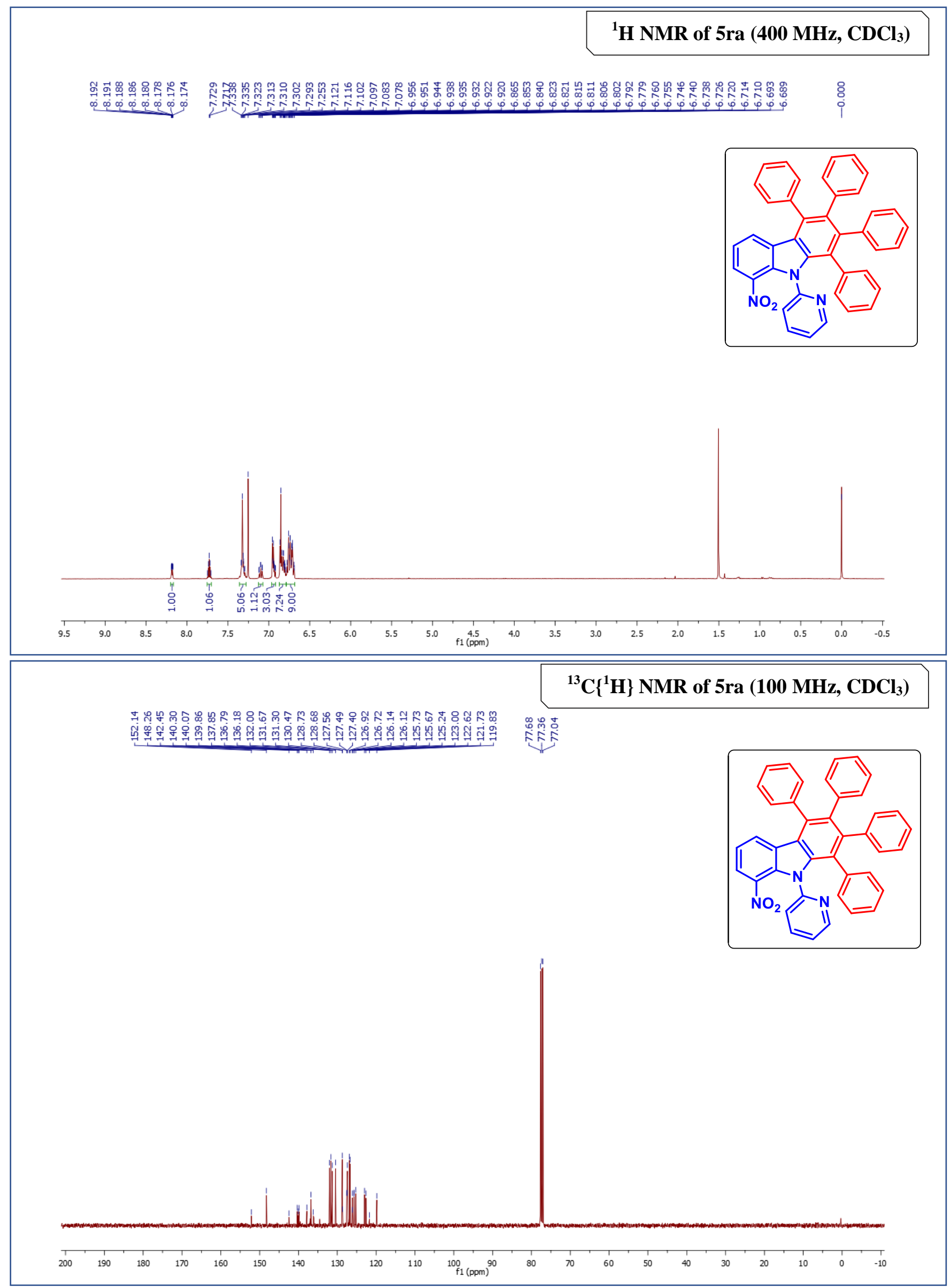




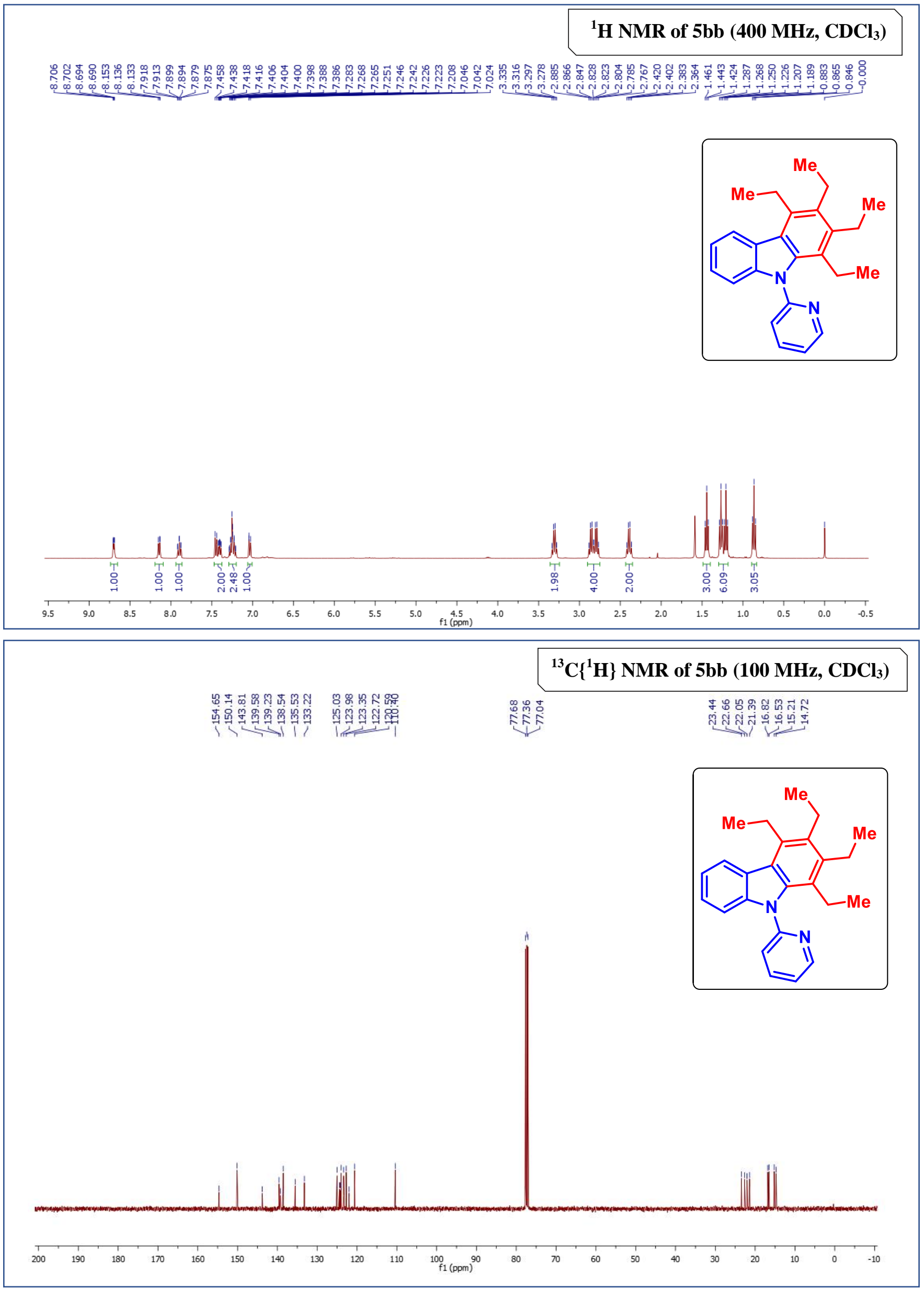




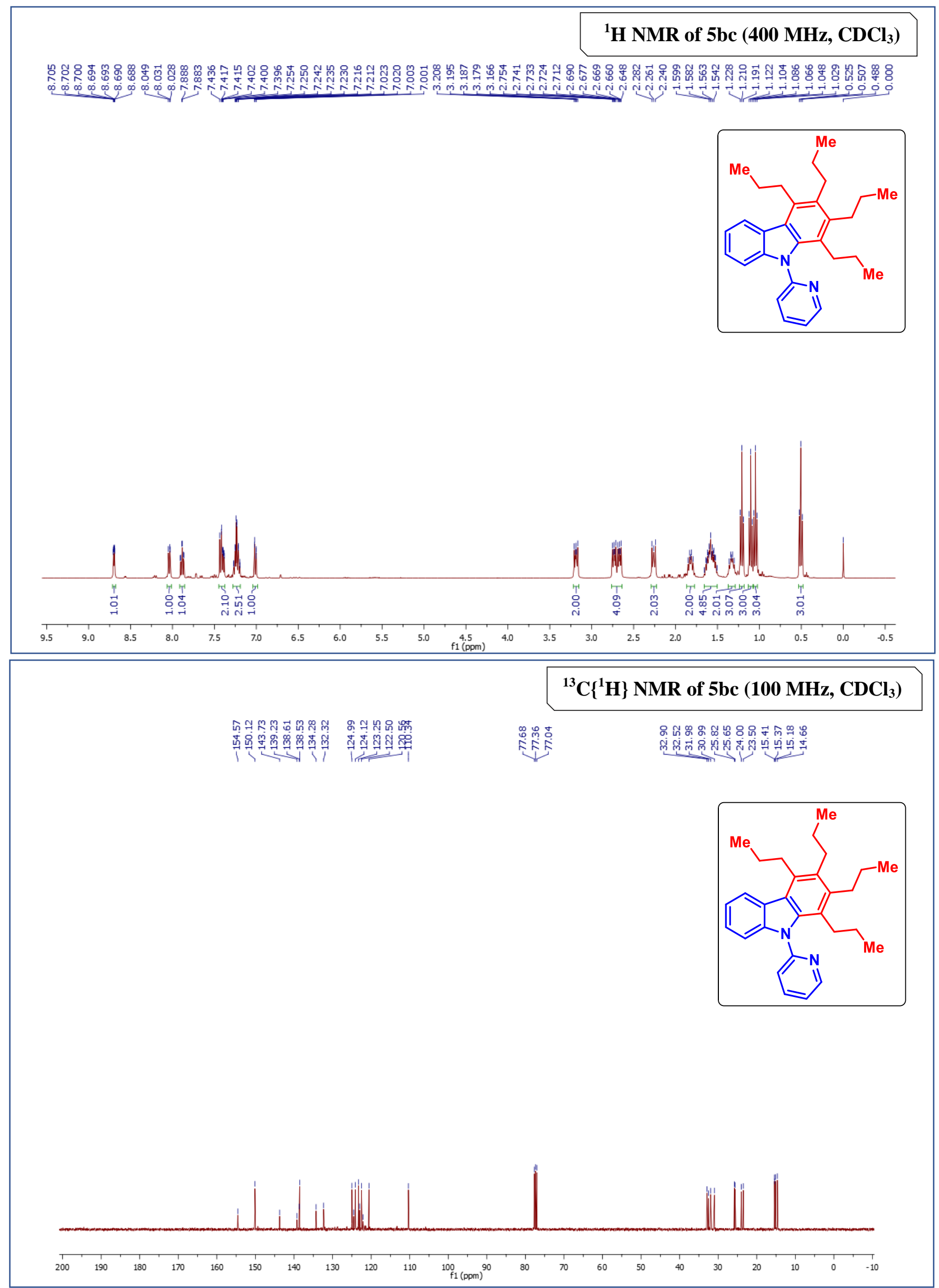




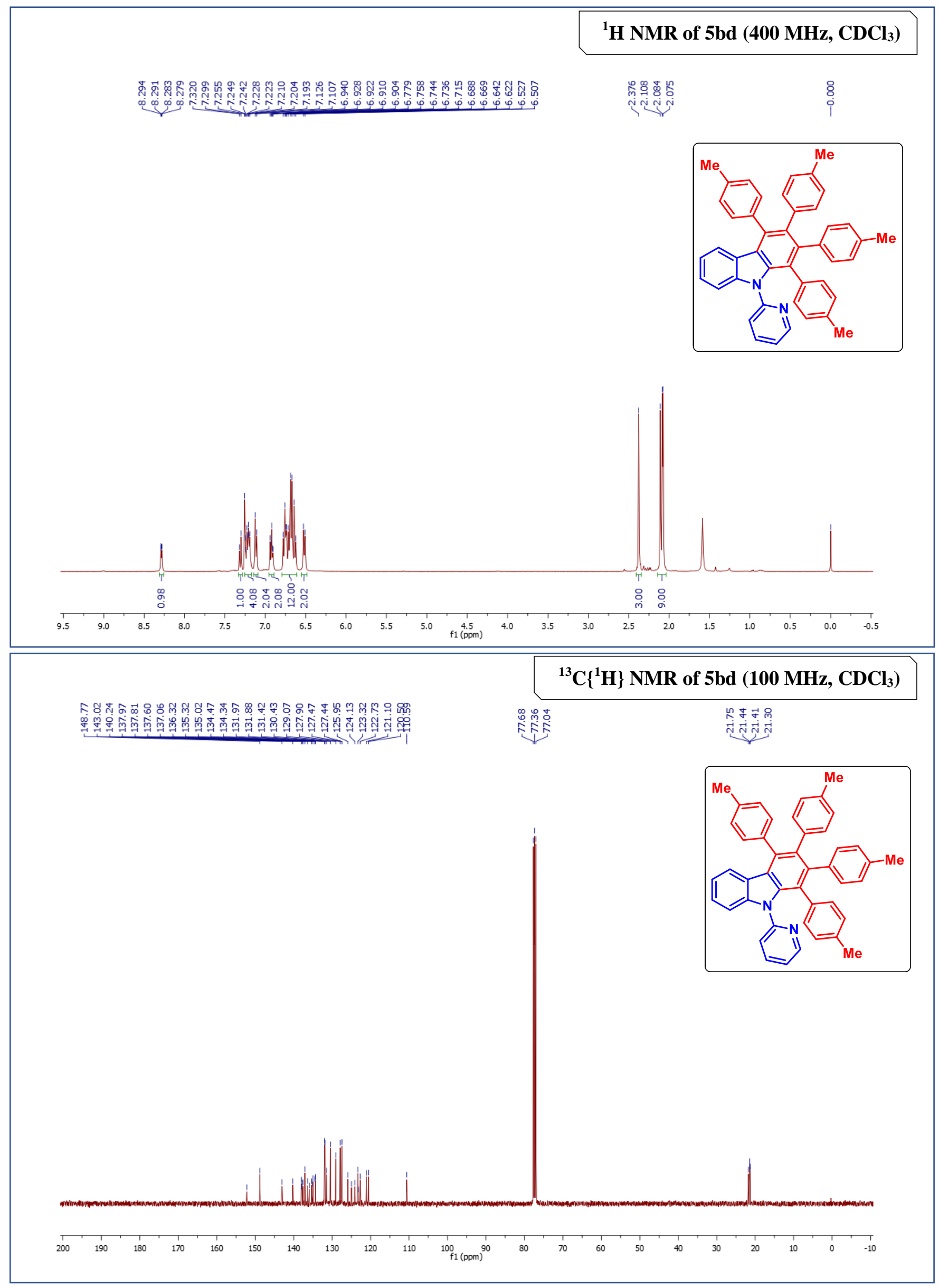




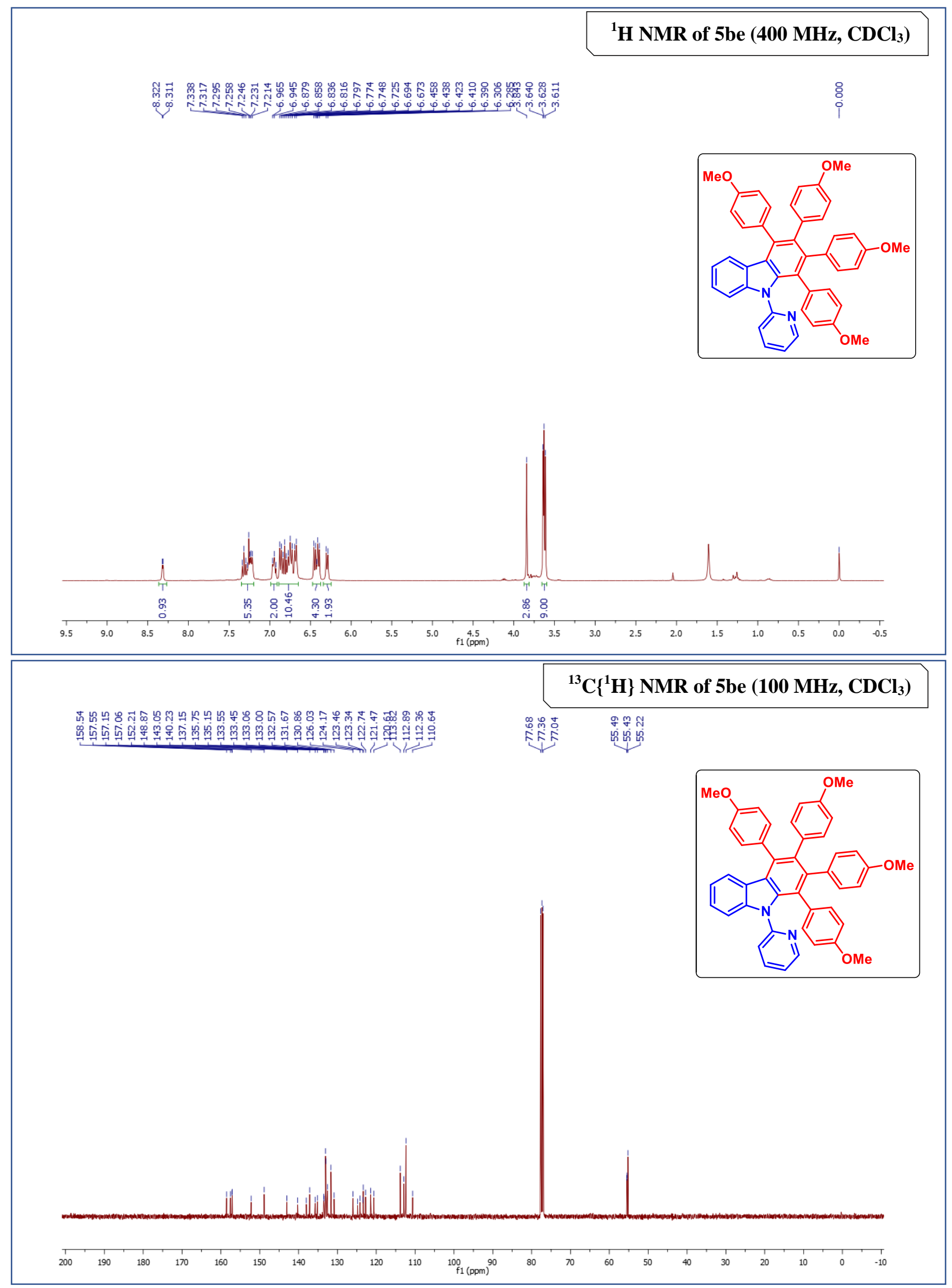



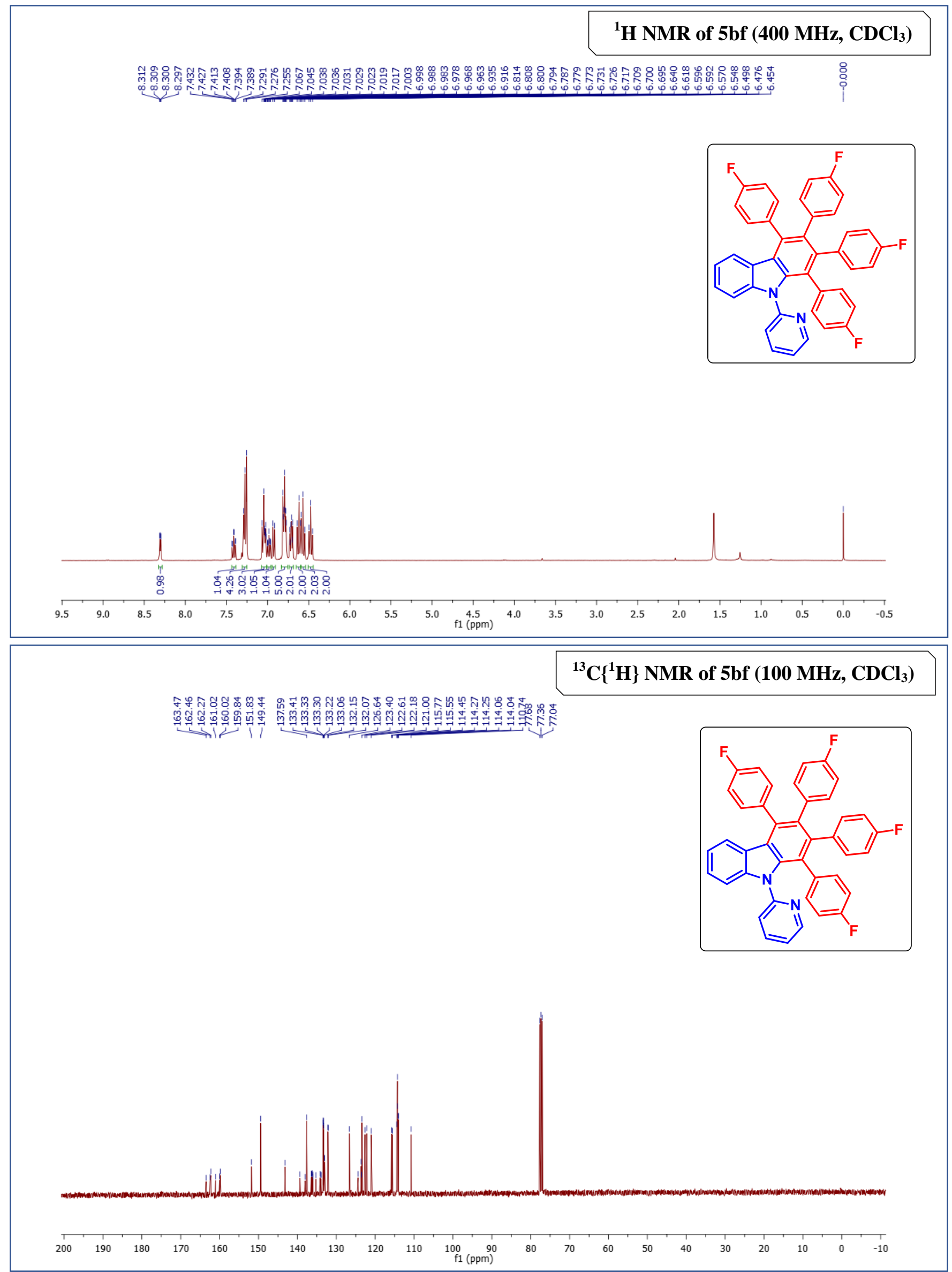


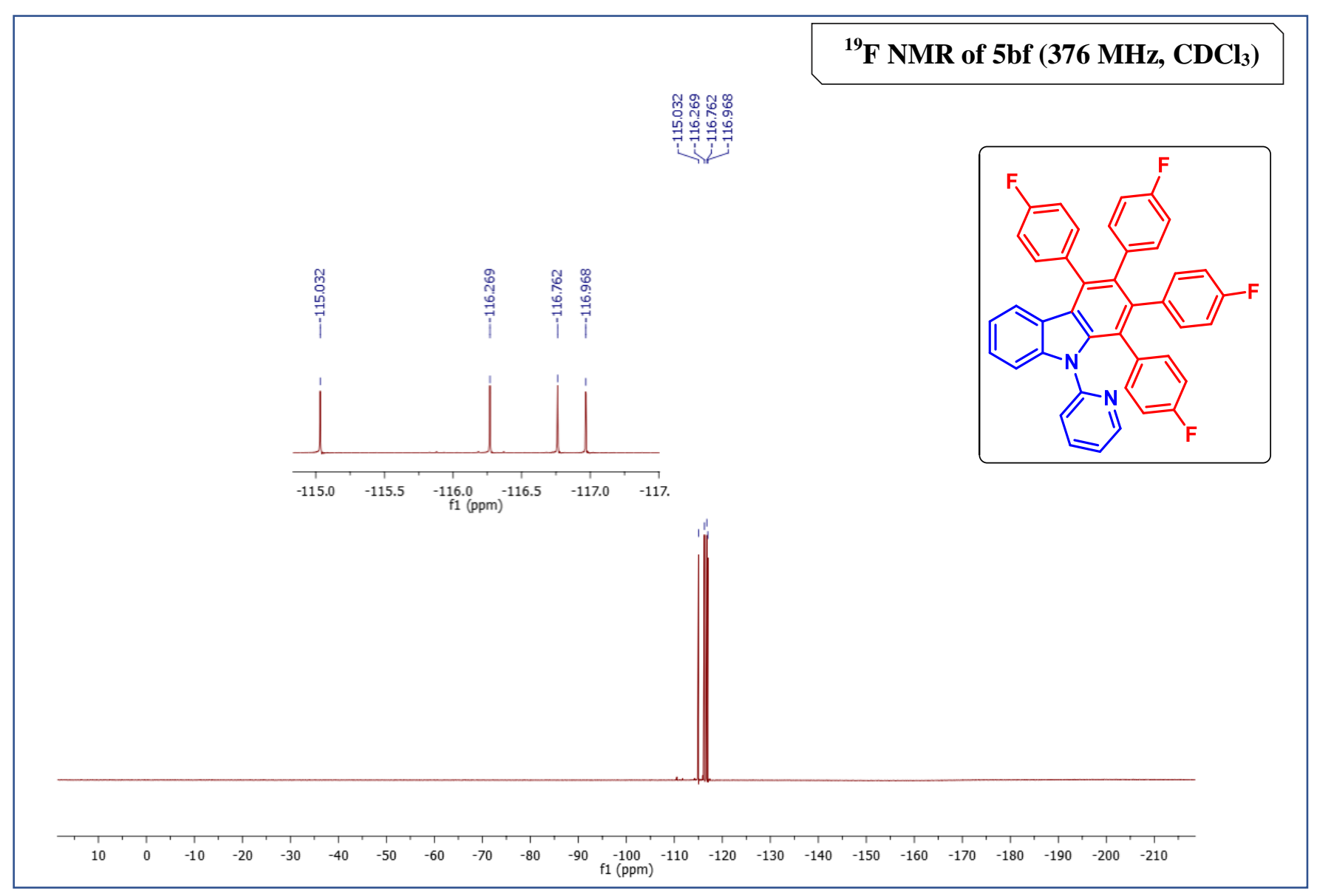

S85 I 


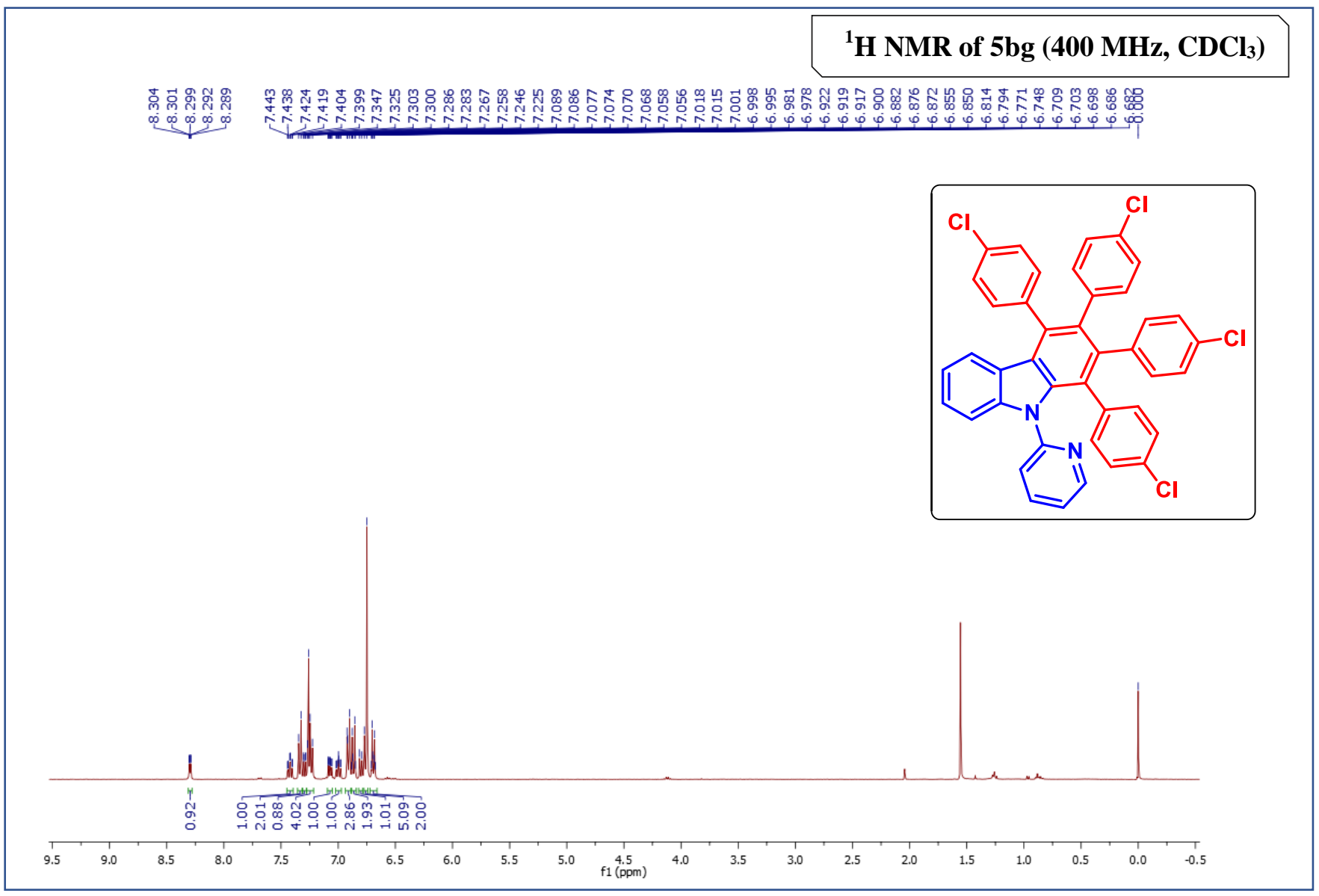

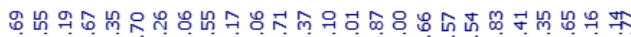

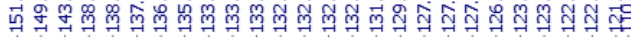

${ }^{13} \mathrm{C}\left\{{ }^{1} \mathrm{H}\right\}$ NMR of $5 \mathrm{bg}\left(100 \mathrm{MHz}, \mathrm{CDCl}_{3}\right)$

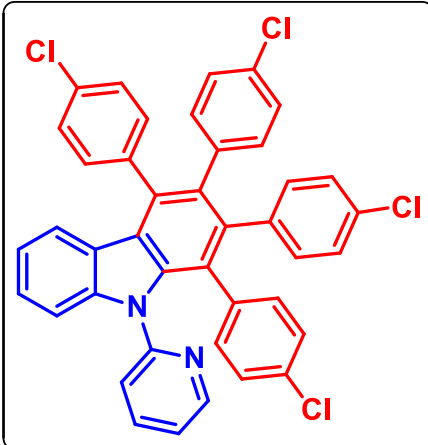




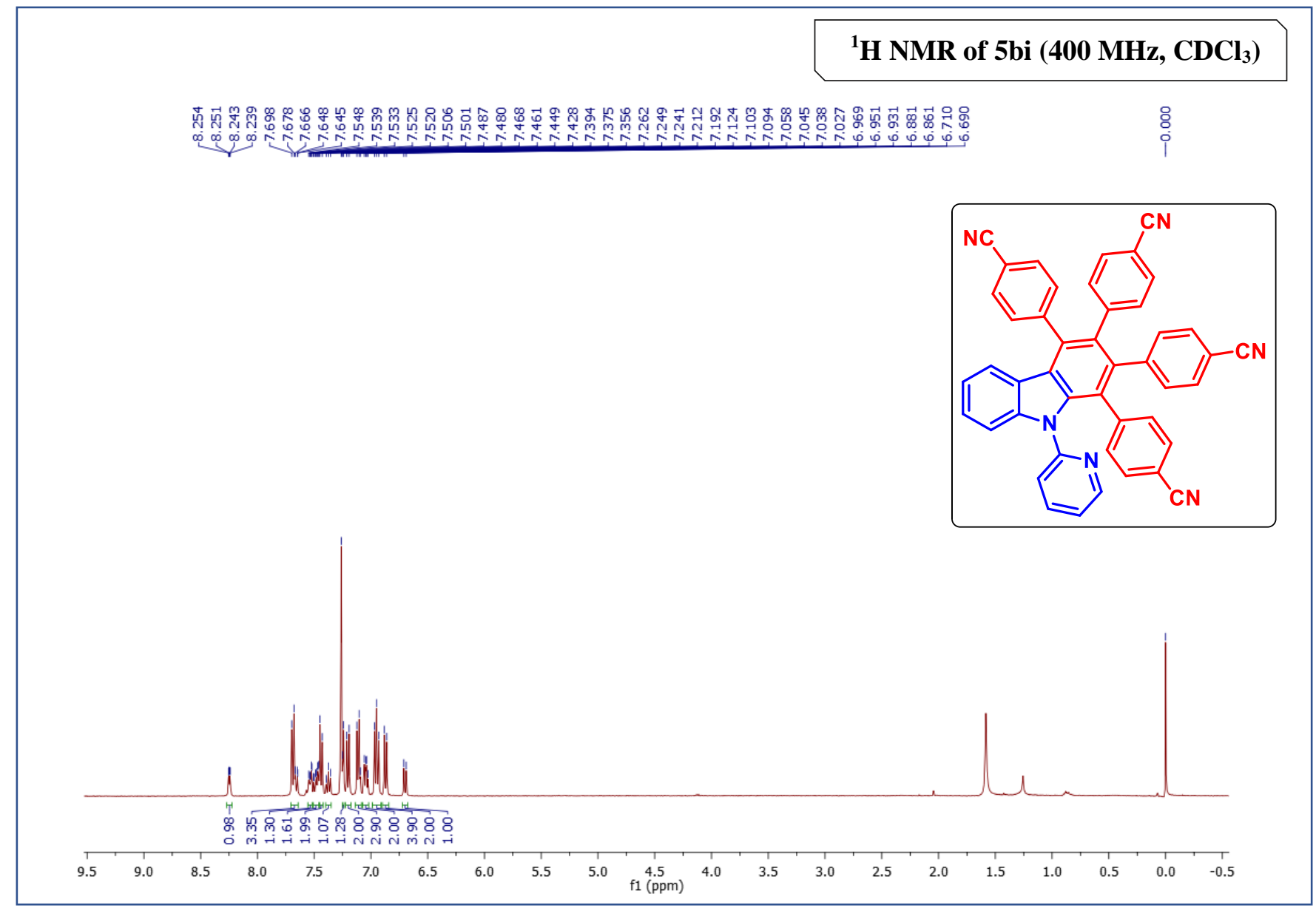

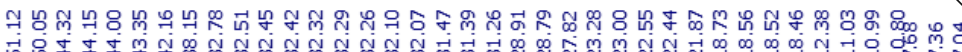

${ }^{13} \mathrm{C}\left\{{ }^{1} \mathrm{H}\right\}$ NMR of $5 \mathrm{bi}\left(100 \mathrm{MHz}, \mathrm{CDCl}_{3}\right)$

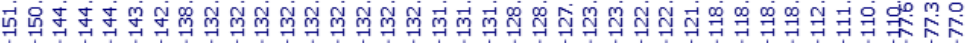

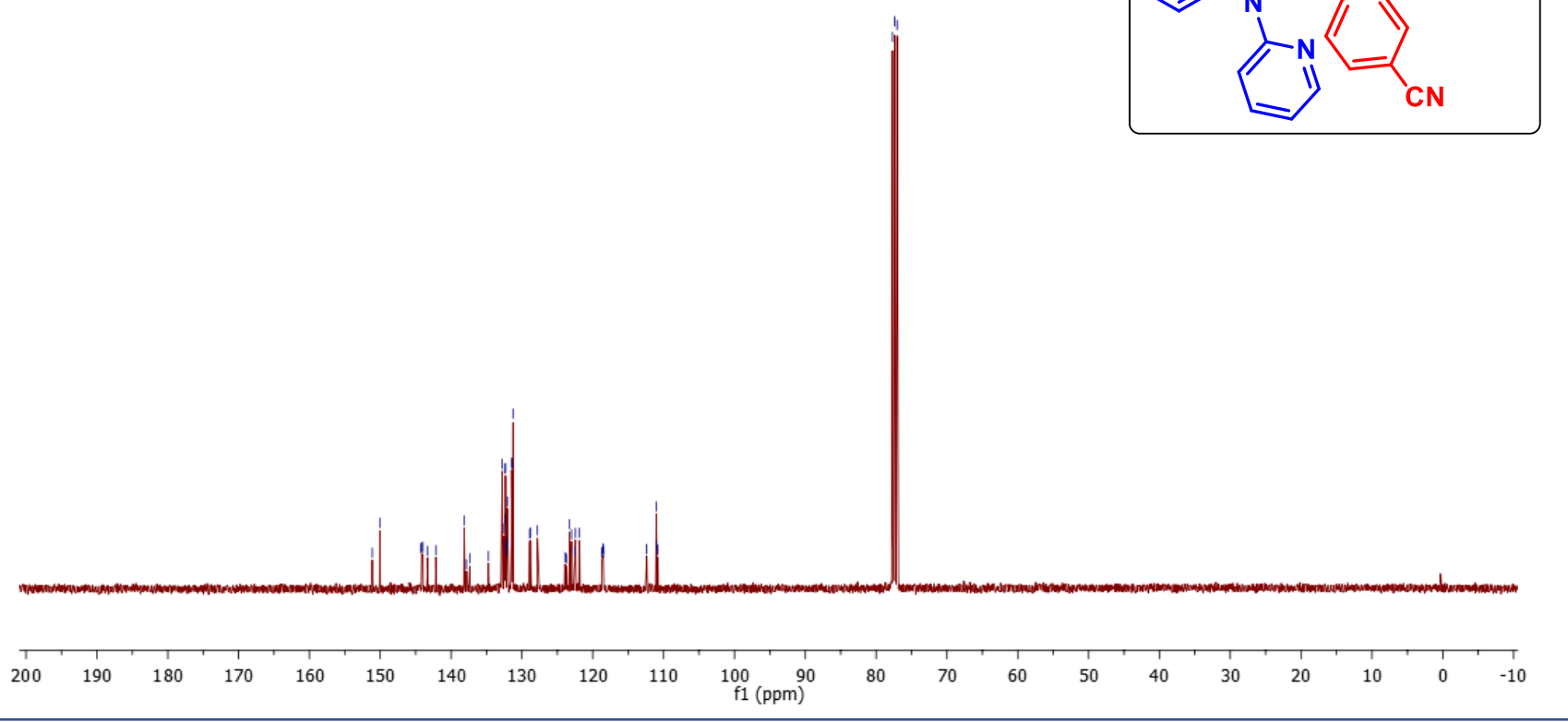




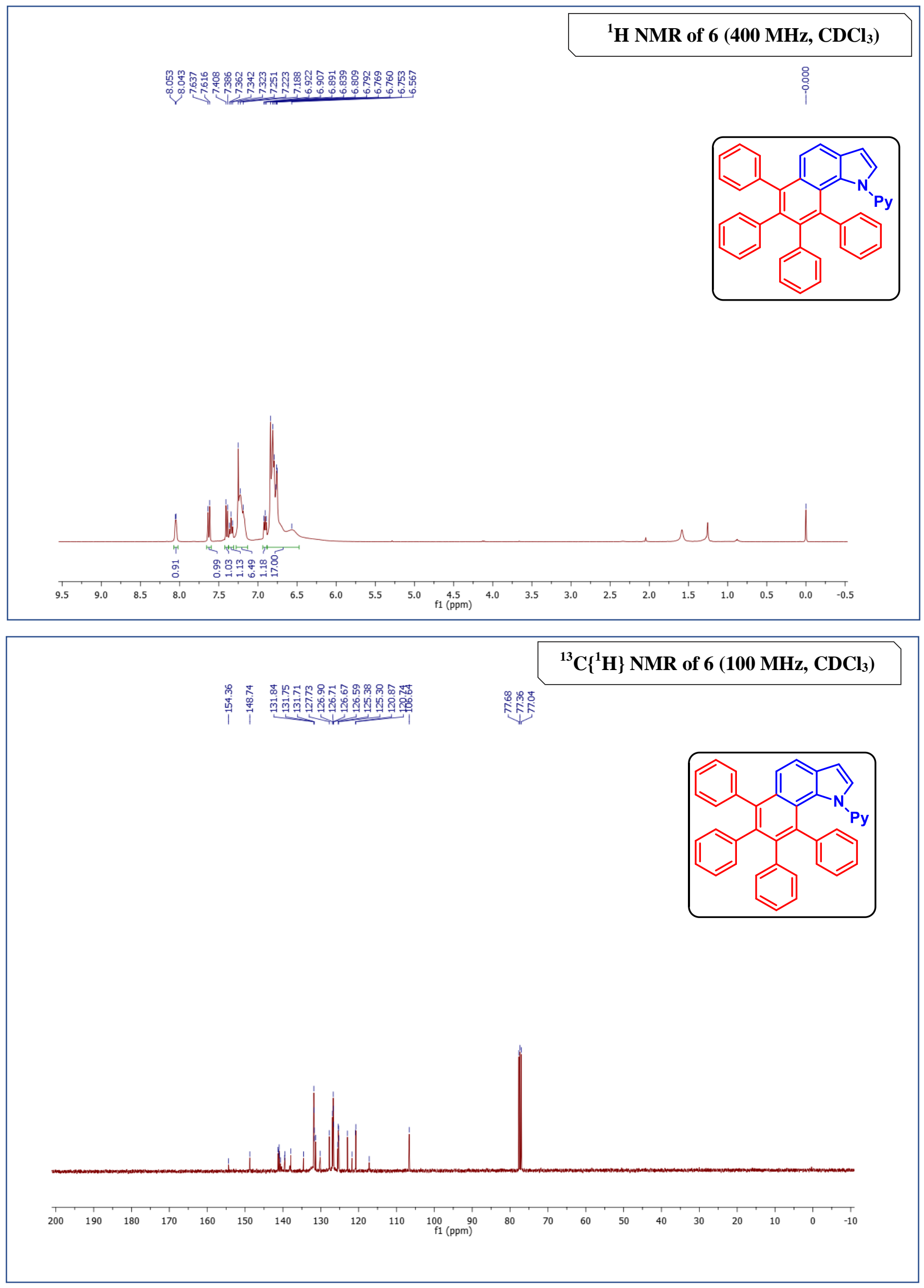



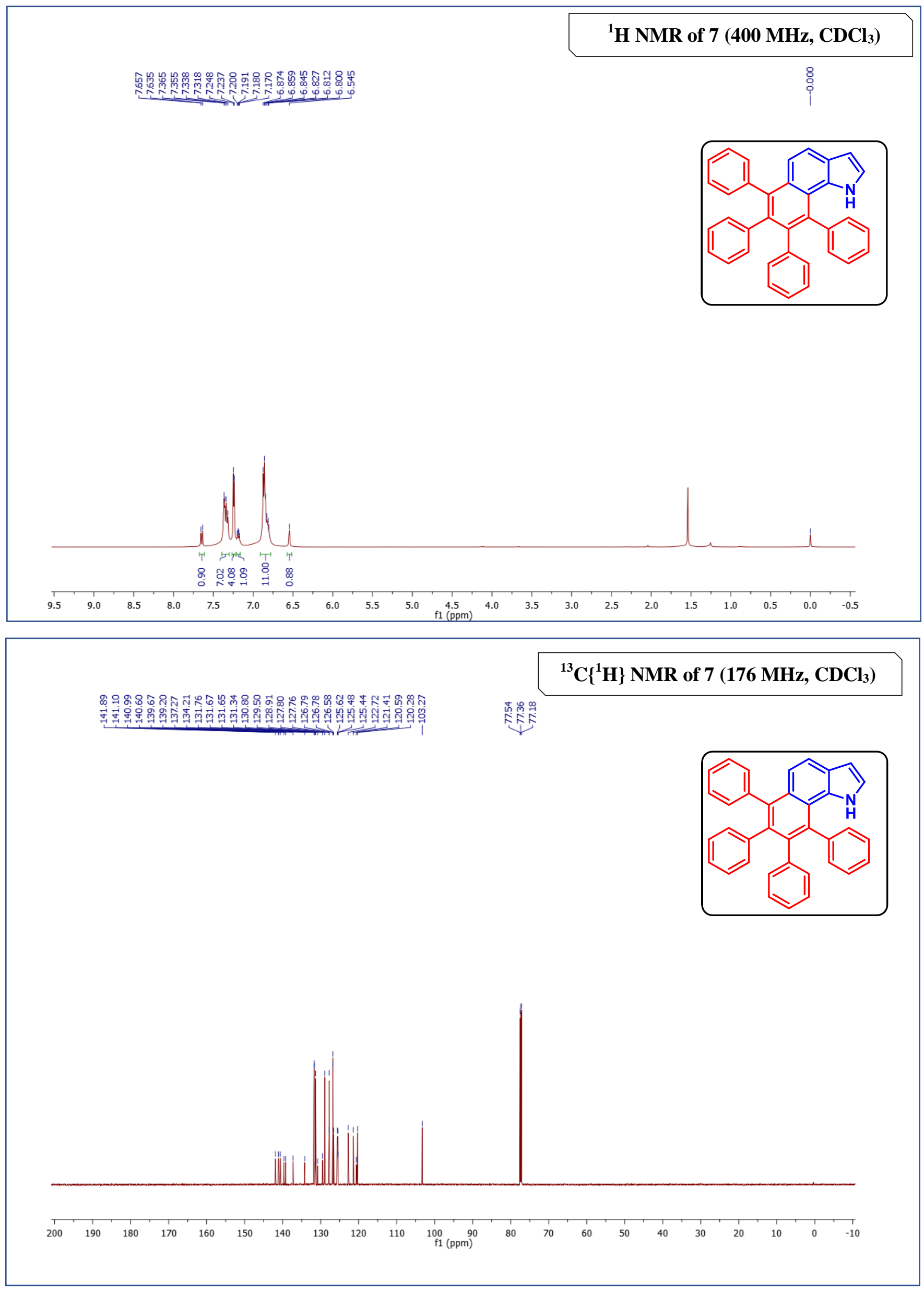


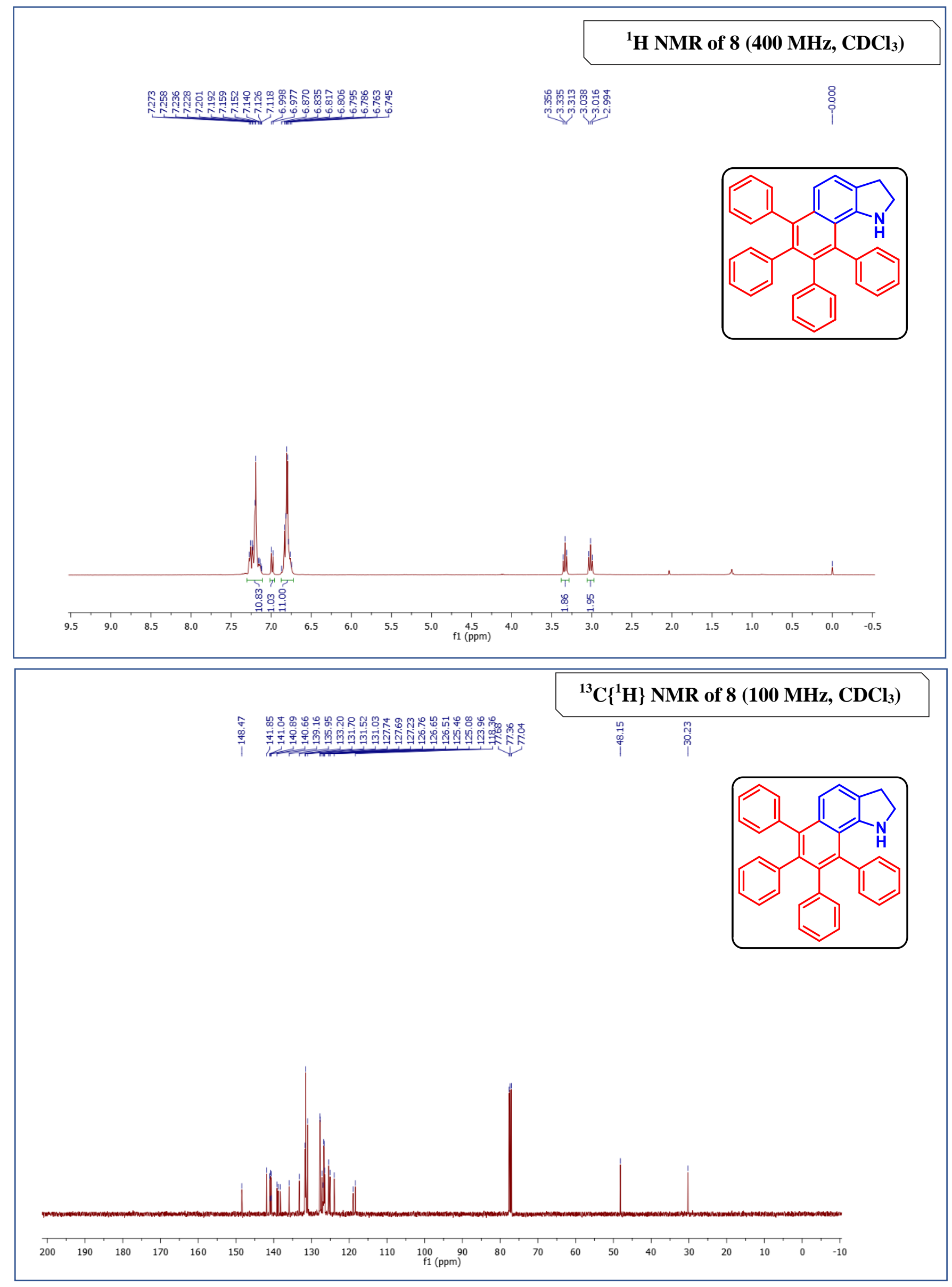




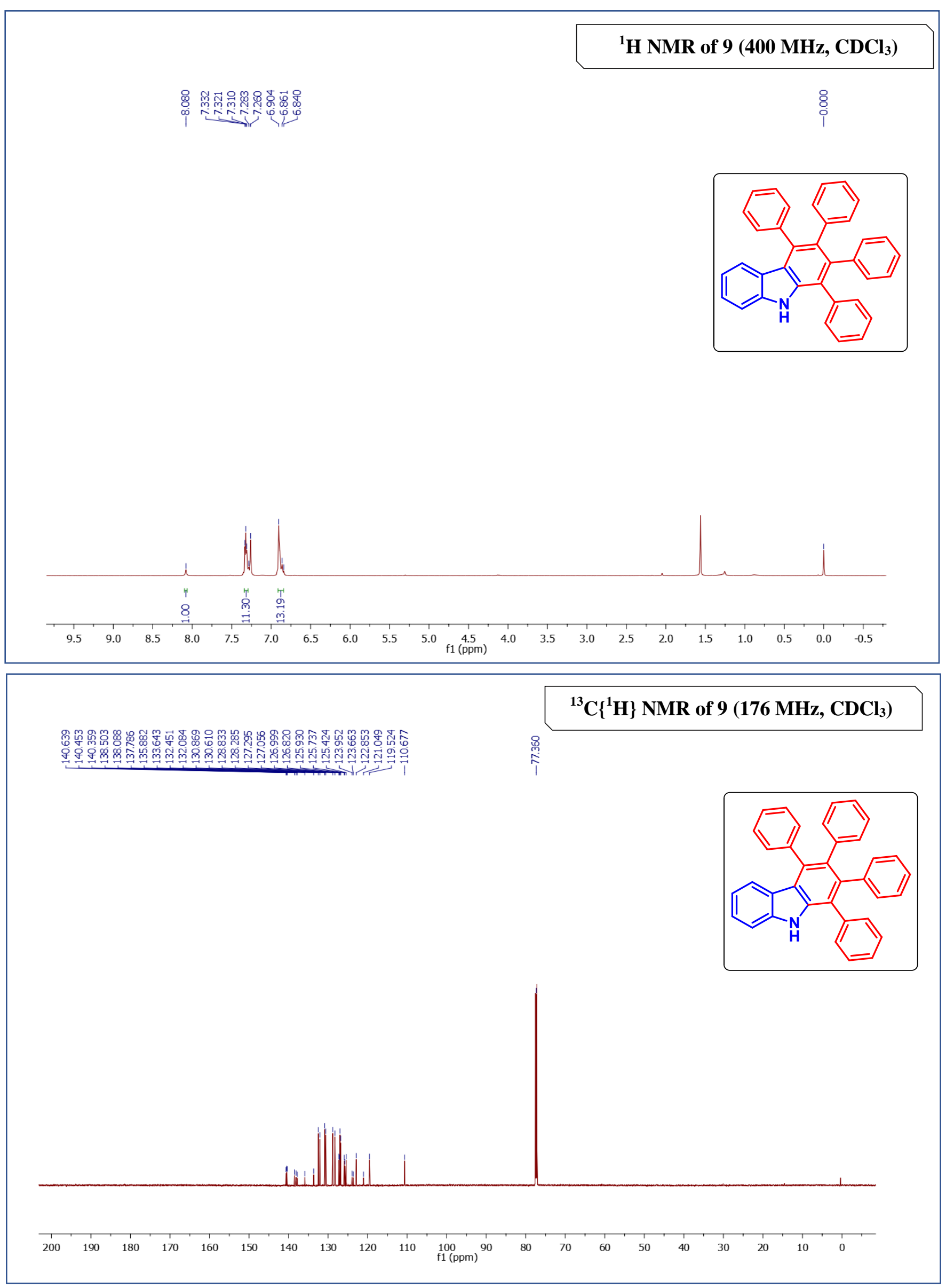




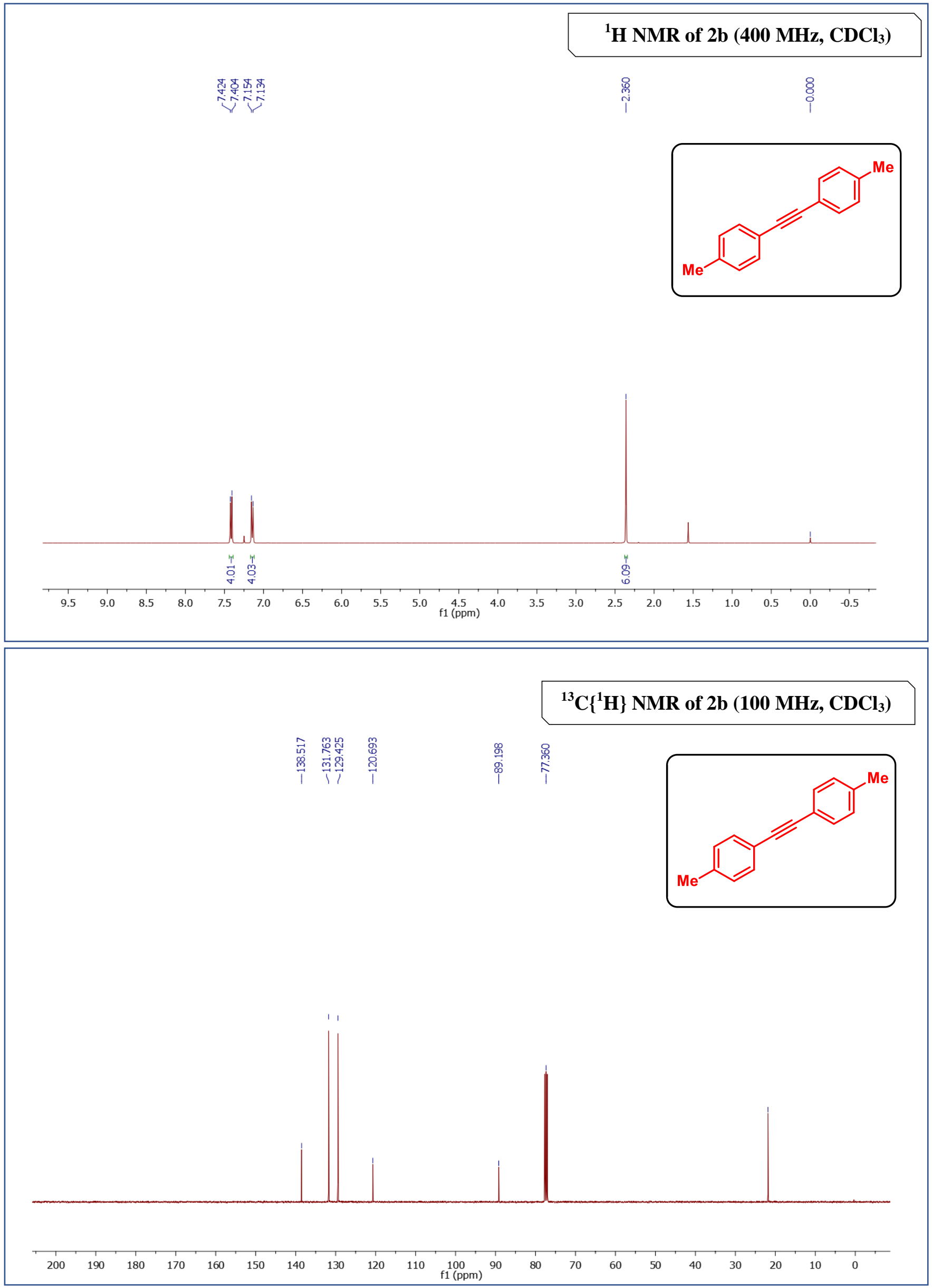



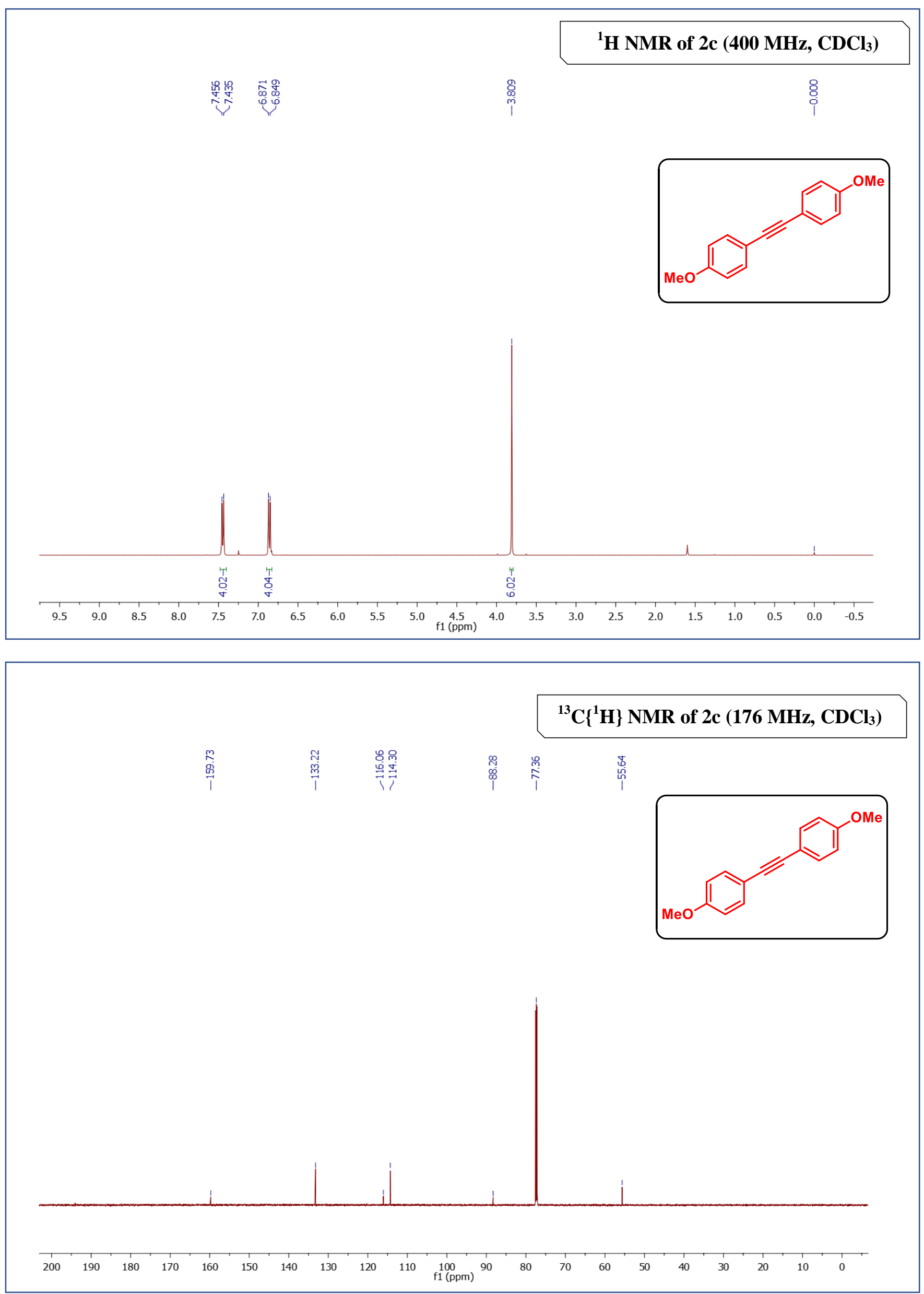

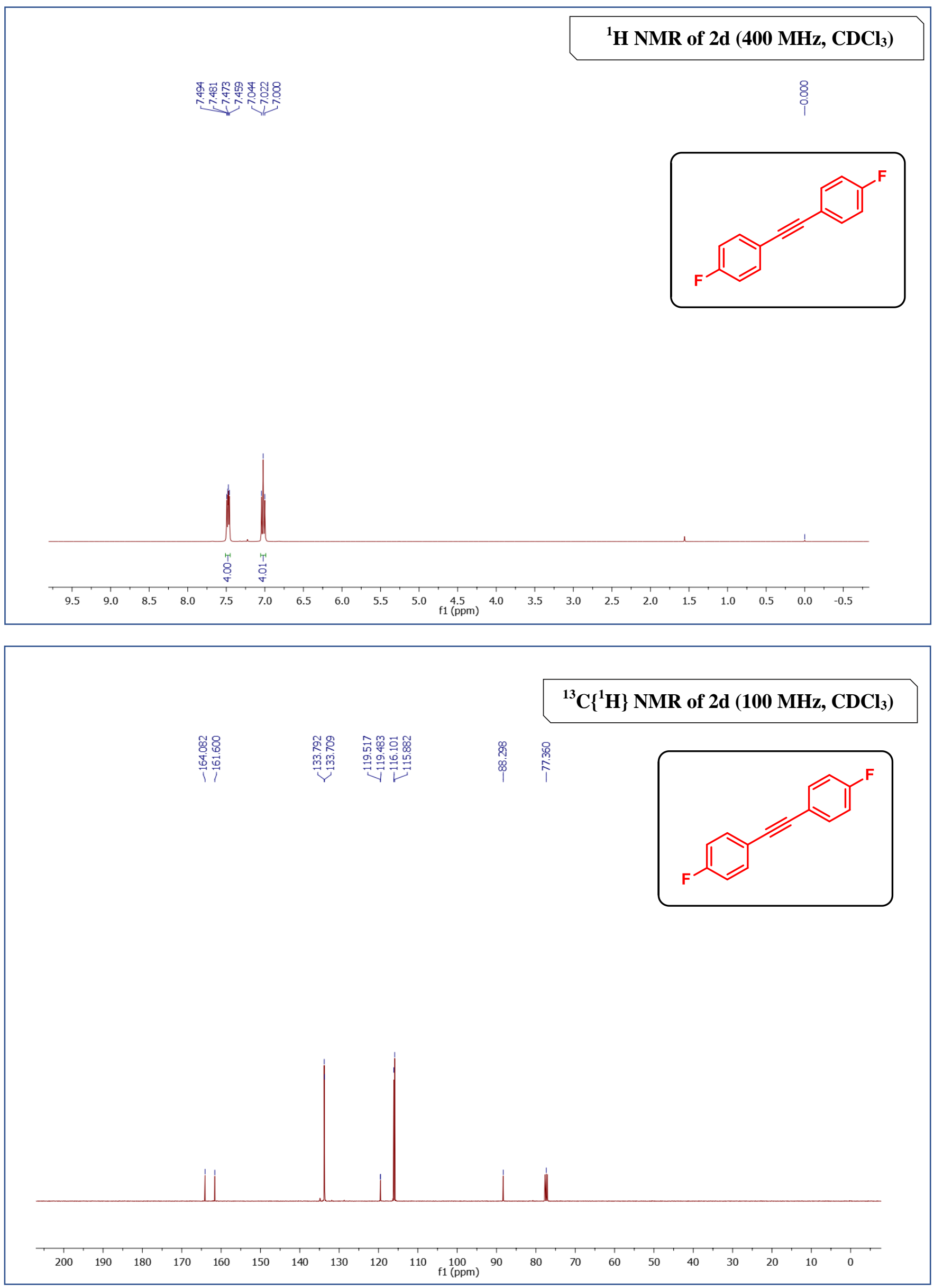


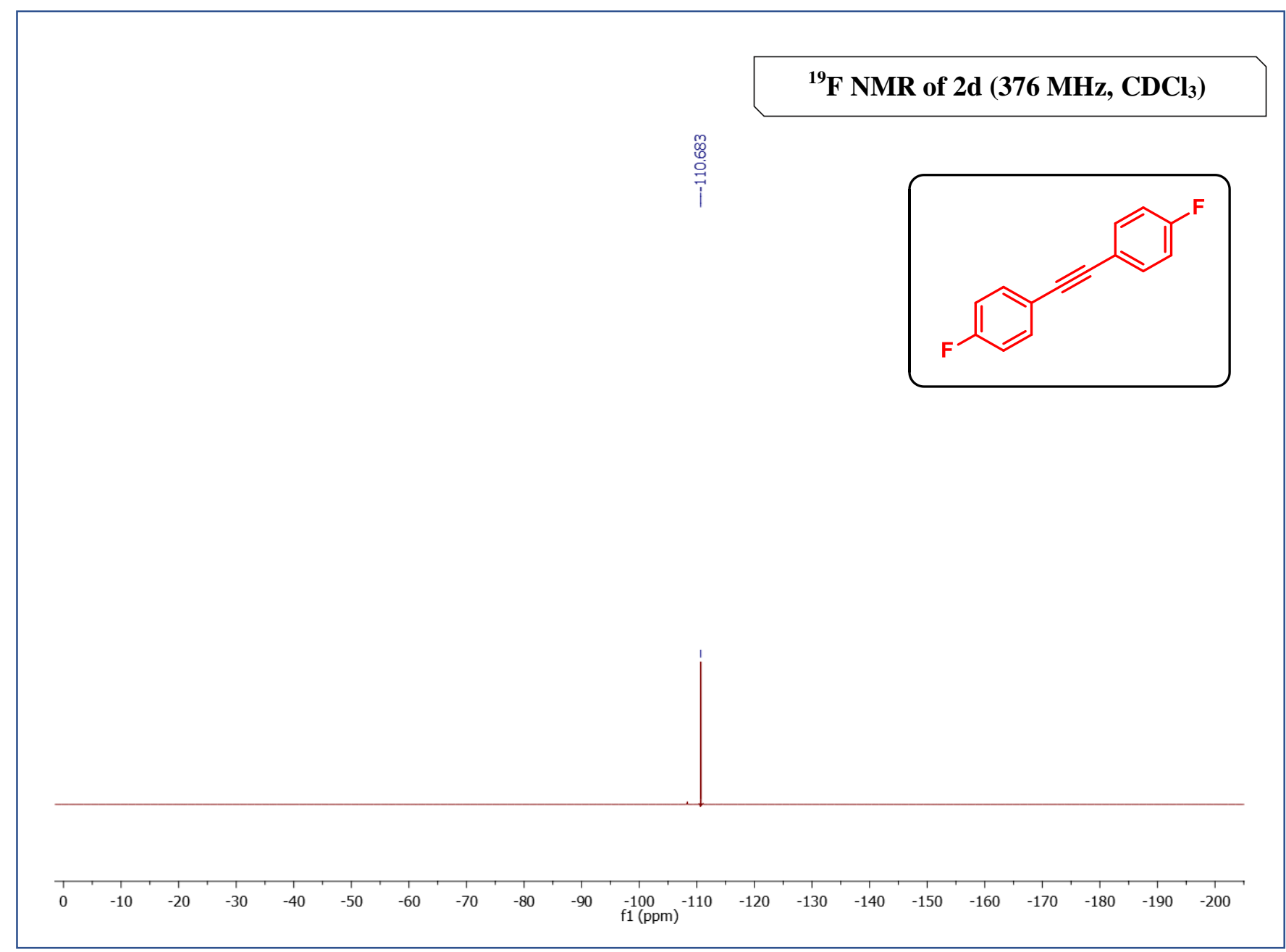

S95 | 

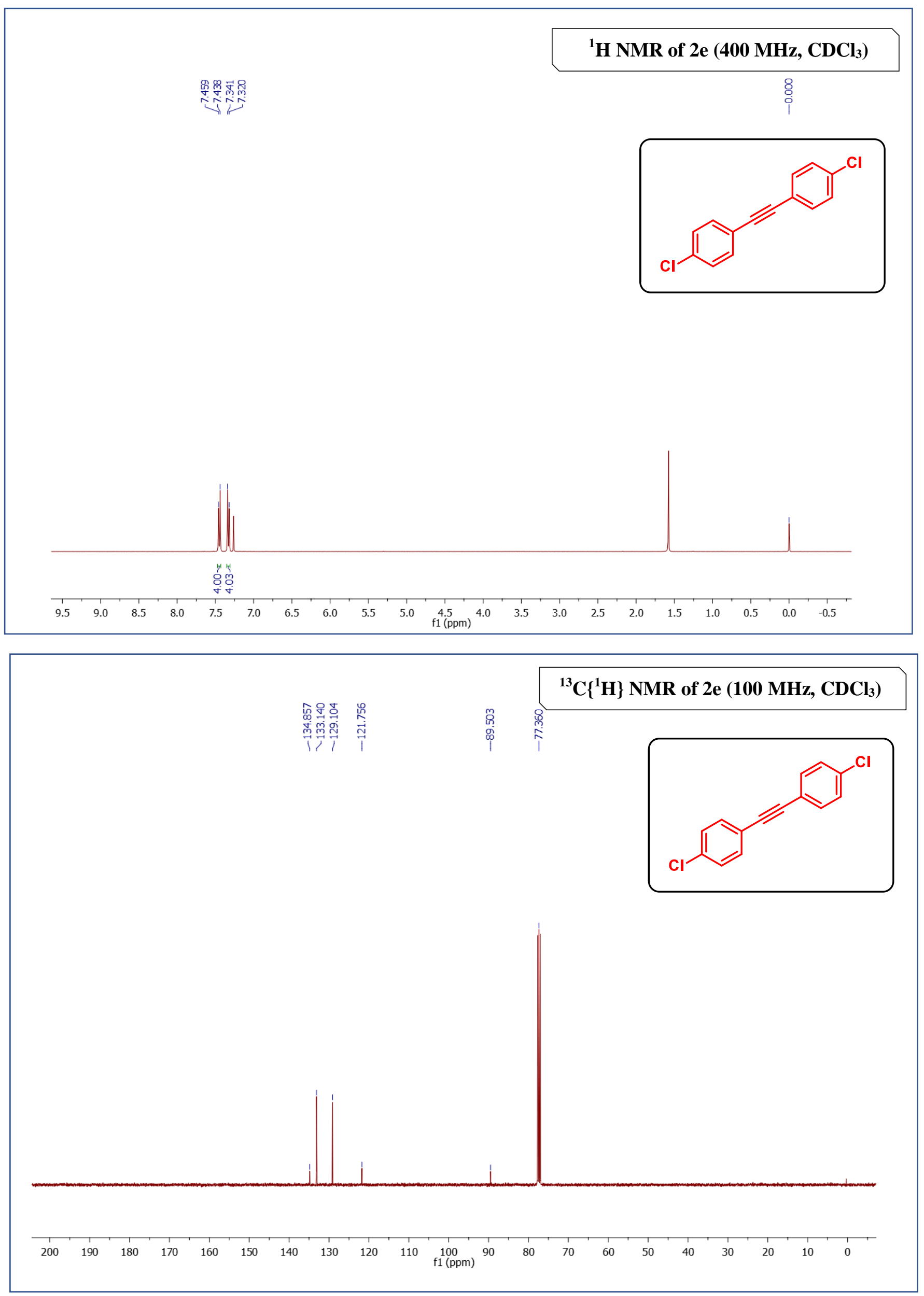


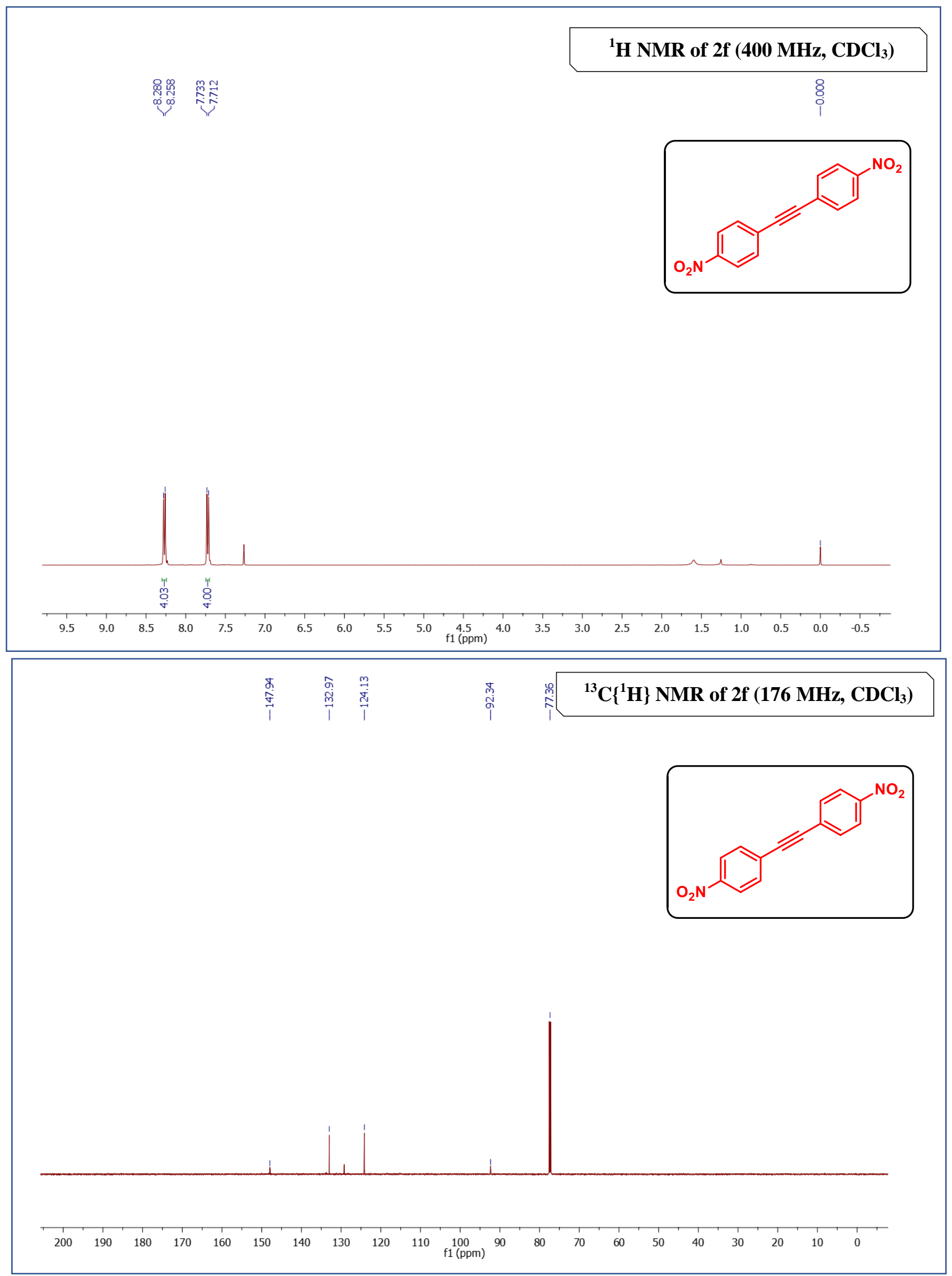




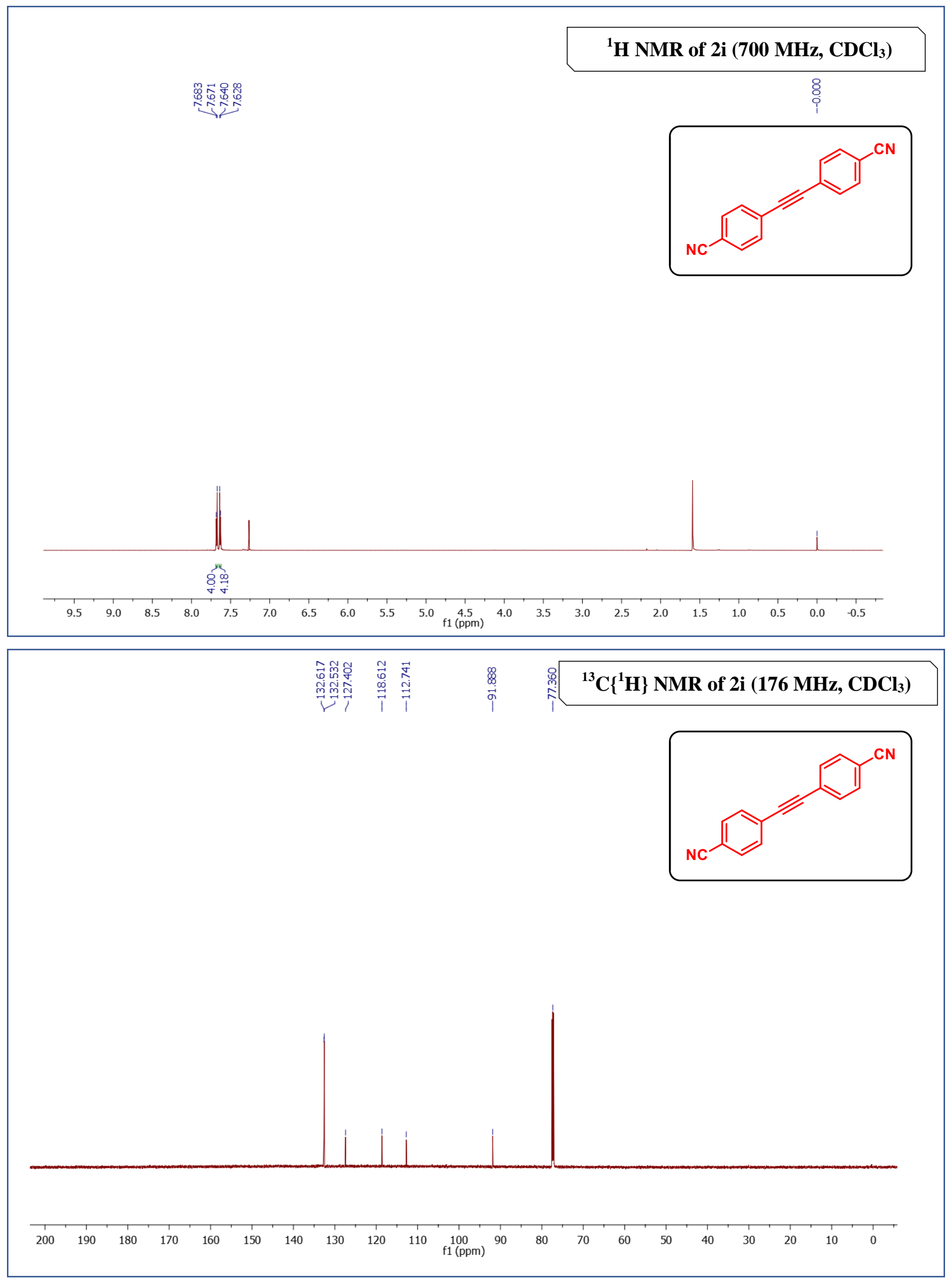




\section{X-ray data:}

\section{(a) X-ray data of 8-nitro-1,2,3,4-tetraphenyl-9-(pyridin-2-yl)-9H-carbazole (5ra):}

Crystals of the compound 5ra were obtained after slow evaporation of EtOAc. Crystals suited for single crystal X-Ray diffraction measurements were mounted on a glass fiber. Geometry and intensity data were collected with a Rigaku Smartlab X-ray diffractometer equipped with graphite-monochromated $\mathrm{Cu}-\mathrm{K} \alpha$ radiation ( $\lambda=1.54184 \AA$, multilayer optics). Temperature was controlled using an Oxford Cryostream 700 instrument. Intensities were integrated with SAINT and SMART software packages and corrected for absorption with SADABS. The structure was solved by direct methods and refined on F2 with SHELXL-97 using Olex-2 software.

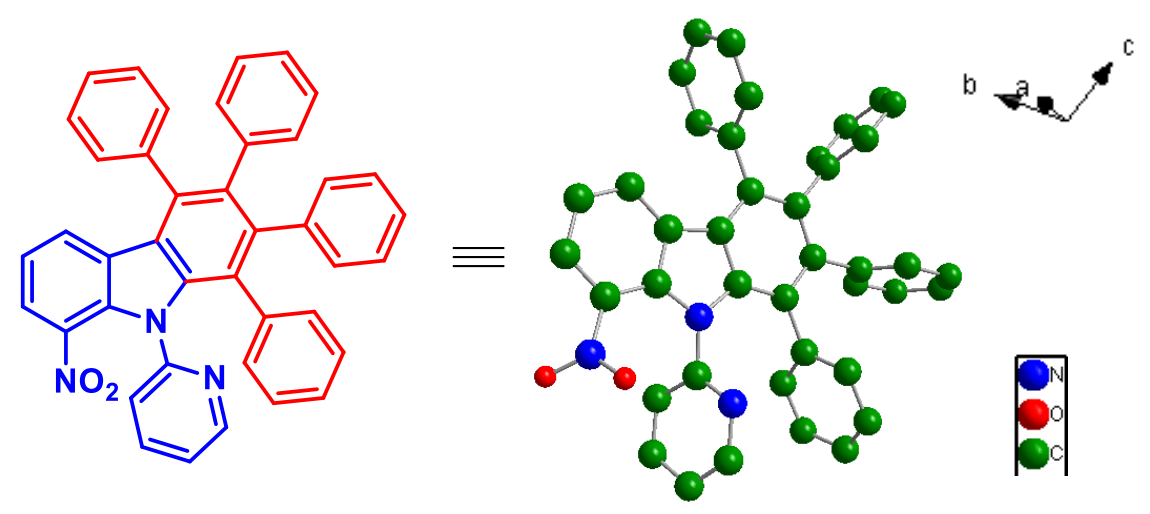

5 ra

CCDC 2041963

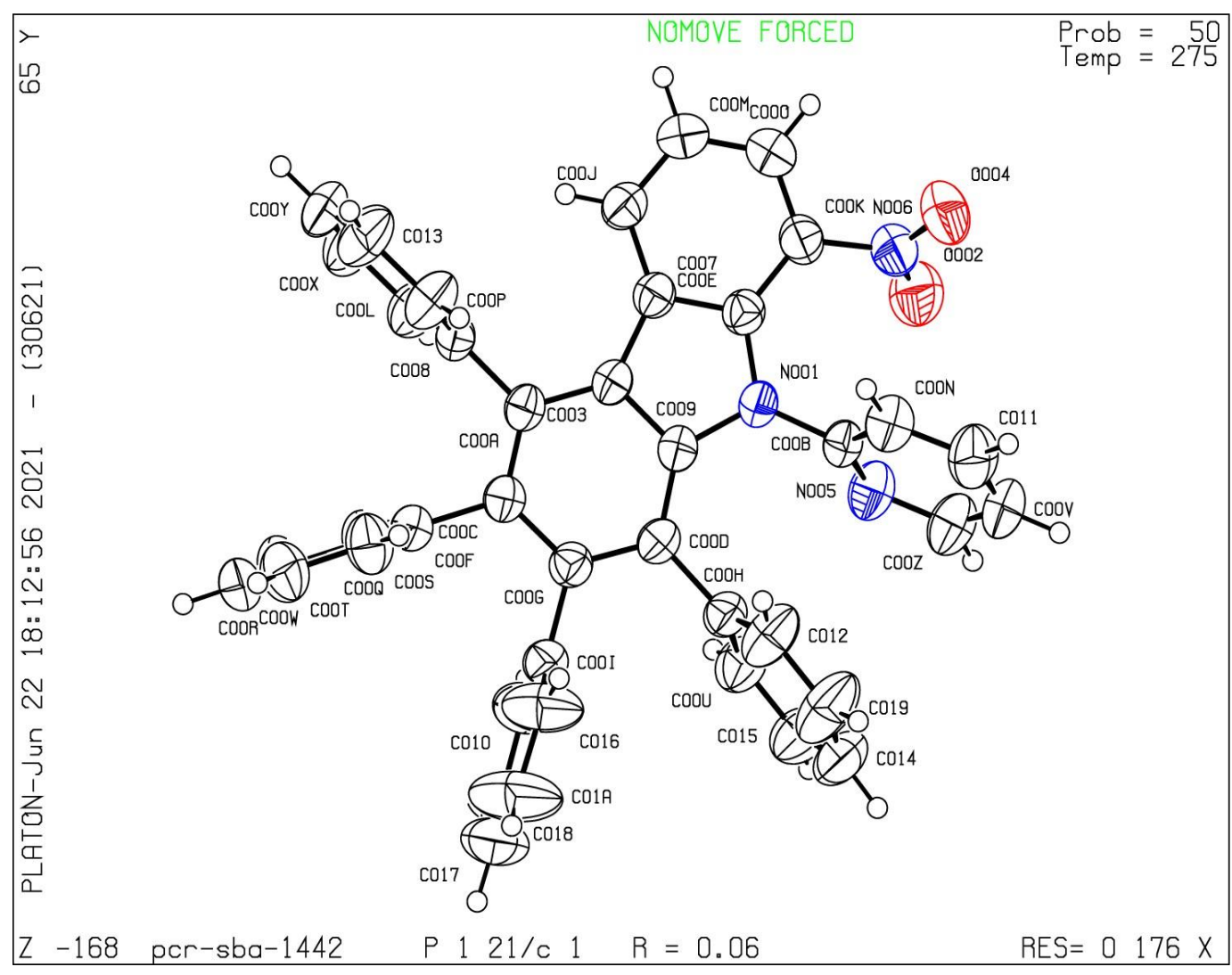

Figure S1. ORTEP diagram of 6 ra with $50 \%$ ellipsoid probability 
Table S1. Crystal data and structure refinement for 8-nitro-1,2,3,4-tetraphenyl-9(pyridin-2-yl)-9H-carbazole (5ra):

\begin{tabular}{|c|c|}
\hline Compound code & Pcr-sba-1442 \\
\hline CCDC Number & 2041963 \\
\hline \multicolumn{2}{|l|}{ Crystal data } \\
\hline Chemical formula & $\mathrm{C}_{41} \mathrm{H}_{27} \mathrm{~N}_{3} \mathrm{O}_{2}$ \\
\hline$M_{\mathrm{r}}$ & $\underline{593.65}$ \\
\hline $\begin{array}{l}\text { Crystal system, space } \\
\text { group }\end{array}$ & $\underline{\text { Monoclinic }}, \underline{P 2_{1}} \underline{\underline{c}}$ \\
\hline Temperature (K) & $\underline{275}$ \\
\hline$a, b, c(\AA)$ & $11.8322(1), 25.3802(3), 12.4568(1)$ \\
\hline$\beta\left({ }^{\circ}\right)$ & $\underline{114.903(1)}$ \\
\hline$V\left(\AA^{3}\right)$ & $\underline{3393.01(6)}$ \\
\hline$Z$ & $\underline{4}$ \\
\hline Radiation type & $\underline{\mathrm{Cu} K \alpha}$ \\
\hline$\mu\left(\mathrm{mm}^{-1}\right)$ & $\underline{0.57}$ \\
\hline Crystal size (mm) & $\underline{0.13} \times \underline{0.12} \times \underline{0.11}$ \\
\hline \multicolumn{2}{|l|}{ Data collection } \\
\hline Diffractometer & SuperNova, Dual, $\mathrm{Cu}$ at home/near, Pilatus $200 \mathrm{~K}$ \\
\hline Absorption correction & $\begin{array}{l}\text { Multi-scan } \\
\text { CrysAlis PRO 1.171.40.84a (Rigaku Oxford Diffraction, 2020) Empirical } \\
\text { absorption correction using spherical harmonics, implemented in } \\
\text { SCALE3 ABSPACK scaling algorithm. }\end{array}$ \\
\hline$T_{\min }, T_{\max }$ & $\underline{0.536}, \underline{1.000}$ \\
\hline $\begin{array}{l}\text { No. of measured, } \\
\text { independent and } \\
\text { observed }[\underline{I>2 \sigma(I)]} \\
\text { reflections }\end{array}$ & $\underline{50962}, \underline{6213}, \underline{5560}$ \\
\hline$R_{\text {int }}$ & $\underline{0.040}$ \\
\hline$(\sin \theta / \lambda)_{\max }\left(\AA^{-1}\right)$ & 0.602 \\
\hline \multicolumn{2}{|l|}{ Refinement } \\
\hline $\begin{array}{l}R\left[F^{2}>2 \sigma\left(F^{2}\right)\right] \\
w R\left(F^{2}\right), S\end{array}$ & $\underline{0.063}, \underline{0.210}, \underline{1.09}$ \\
\hline No. of reflections & $\underline{6213}$ \\
\hline No. of parameters & $\underline{415}$ \\
\hline H-atom treatment & $\underline{\text { H-atom parameters constrained }}$ \\
\hline
\end{tabular}

